\title{
Near infrared fluorescence imaging in surgery
}

\author{
Citation for published version (APA):
}

van den Bos, J. (2019). Near infrared fluorescence imaging in surgery: seeing more? [Doctoral Thesis, Maastricht University]. ProefschriftMaken Maastricht. https://doi.org/10.26481/dis.20191206jb

\section{Document status and date:}

Published: 01/01/2019

DOI:

10.26481/dis.20191206jb

Document Version:

Publisher's PDF, also known as Version of record

\section{Please check the document version of this publication:}

- A submitted manuscript is the version of the article upon submission and before peer-review. There can be important differences between the submitted version and the official published version of record.

People interested in the research are advised to contact the author for the final version of the publication, or visit the DOI to the publisher's website.

- The final author version and the galley proof are versions of the publication after peer review.

- The final published version features the final layout of the paper including the volume, issue and page numbers.

Link to publication

\footnotetext{
General rights rights.

- You may freely distribute the URL identifying the publication in the public portal. please follow below link for the End User Agreement:

www.umlib.nl/taverne-license

Take down policy

If you believe that this document breaches copyright please contact us at:

repository@maastrichtuniversity.nl

providing details and we will investigate your claim.
}

Copyright and moral rights for the publications made accessible in the public portal are retained by the authors and/or other copyright owners and it is a condition of accessing publications that users recognise and abide by the legal requirements associated with these

- Users may download and print one copy of any publication from the public portal for the purpose of private study or research.

- You may not further distribute the material or use it for any profit-making activity or commercial gain

If the publication is distributed under the terms of Article $25 \mathrm{fa}$ of the Dutch Copyright Act, indicated by the "Taverne" license above, 


\section{Near infrared fluorescence imaging in surgery Seeing more?}


(c) 2019 Jacqueline van den Bos

All rights reserved. No part of this thesis may be reproduced or distributed in any form or by any means, without the prior written permission of the author or publisher.

Lay-out: RON Graphic Power \| www.ron.nu

Cover design: Jacqueline van den Bos

Printed by: ProefschriftMaken || www.proefschriftmaken.nl

ISBN/EAN: 978-94-6380-615-2

Publication of this thesis was financially supported by Maastricht University, Department of Surgery of the Maastricht University Medical Centre

The work presented in this thesis was performed within NUTRIM School of Nutrition and Translational Research in Metabolism. 


\title{
Near infrared fluorescence imaging in surgery Seeing more?
}

\author{
PROEFSCHRIFT \\ van het College van Decanen, \\ in het openbaar te verdedigen \\ op \\ Vrijdag 6 december 2019 om 12:00 uur \\ door \\ Jacqueline van den Bos \\ geboren op 24 april 1990 \\ te Leiderdorp
}

Ter verkrijging van de graad van doctor aan de Universiteit Maastricht, op gezag van Rector Magnificus prof. dr. Rianne M. Letschert volgens het besluit 


\section{Promotores}

Professor dr. L.P.S. Stassen

Professor dr. N.D. Bouvy

\section{Co-promotor}

Dr. R.M. Schols

\section{Beoordelingscommissie}

Professor dr. S.W.M. Olde Damink (voorzitter)

Professor dr. J.F. Lange

Professor dr. R.R.W.J. van der Hulst

Professor dr. I.B. Tan

Dr. J.P. Ruurda 


\section{Table of contents}

Chapter 1 Introduction and outline of the thesis

PART I NEAR INFRARED FLUORESCENCE CHOLANGIOGRAPHY

Chapter 2 Near-infrared fluorescence cholangiography assisted laparoscopic cholecystectomy versus conventional laparoscopic cholecystectomy (FALCON trial): study protocol for a multicenter randomized controlled trial

BMJ Open. 2016;6(8):e011668.

Chapter 3 Optimizing the image of Fluorescence Cholangiography using ICG:

a systematic review and ex vivo experiments

Surgical Endoscopy 2018; 32(12):4820-32

Chapter 4 Near infrared fluorescence laparoscopy of the cystic duct and cystic artery: first experience with two new preclinical dyes in a pig model

Surgical Endoscopy 2017;31(10):4309-14

Chapter 5 Technical note: measuring the fluorescence intensity in NIRF cholangiography

Journal of laparoendoscopic \& advanced surgical techniques 2019 [Epub ahead of print]

PART II NEAR INFRARED FLUORESCENCE IMAGING IN COLORECTAL SURGERY

Chapter 6 Near-Infrared fluorescence imaging for real-time intraoperative guidance in anastomotic colorectal surgery: a systematic review of literature 101 Journal of laparoendoscopic \& advanced surgical techniques 2017 28(2):157-167 

Chapter 7 Near-Infrared fluorescence imaging in anastomotic surgery:

is fluorescence intensity related to anastomotic leakage?

Surgical Endoscopy 2019 [Epub Ahead of print]

Chapter 8 Fluorescence ureteral visualization in human laparoscopic colorectal surgery using methylene blue 139

Journal of laparoendoscopic and advanced surgical techniques 2016;

26(11):870-75

Chapter 9 Near-infrared fluorescence laparoscopy of the ureter with three preclinical dyes in a pig model

Surgical Endoscopy 2019 33(3):986-991

Chapter 10 Evaluation of a novel dye for near-infrared fluorescence delineation of the ureters during laparoscopy

BJS Open 2018 DOI: 10.1002/bjs5.59

PART III NEAR INFRARED FLUORESCENCE IMAGING IN THYROID SURGERY

Chapter 11 Feasibility of the use of NIRF in parathyroid identification

Head and Neck 2019 41(2):340-348

PART IV SUMMARY, GENERAL DISCUSSION AND FUTURE PERSPECTIVES

Chapter 12 Summary and general discussion

\section{PART V ADDENDUM}

Nederlandse Samenvatting $\quad 218$

Valorisation $\quad 225$

Dankwoord 228

List of Publications $\quad 232$

$\begin{array}{ll}\text { Curriculum vitae } & 234\end{array}$ 


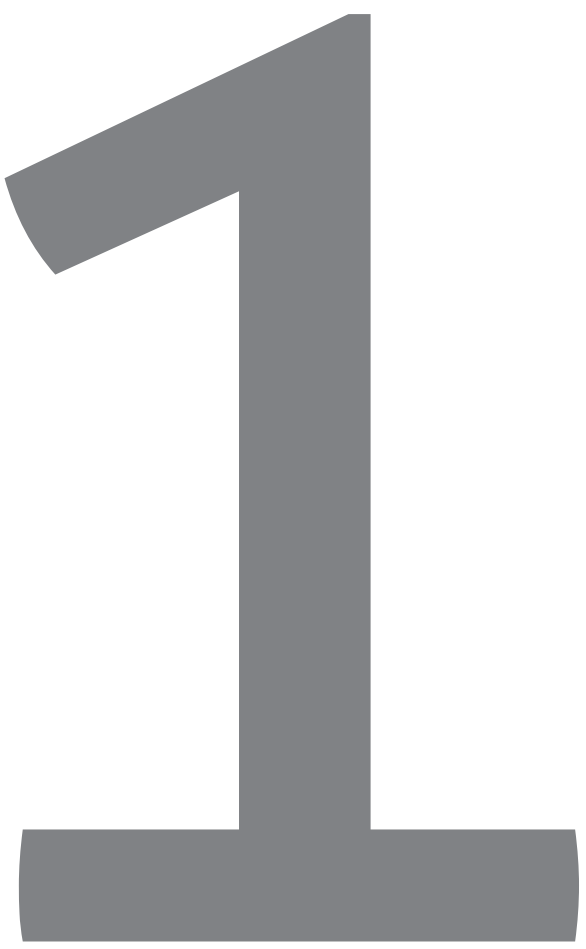


Introduction and outline of the thesis 


\section{INTRODUCTION}

Surgery is the cornerstone of medical treatment of numerous benign conditions such as gallstone disease, but also of malignancies such as colorectal and thyroid cancer. Every surgical procedure does incorporate a risk for complications., ${ }^{1,2}$ Part of these complications occur during surgery and are caused by incorrect identification of vital anatomical structures. For example, with respect to the most frequently performed laparoscopic procedure, laparoscopic cholecystectomy, bile duct injury is the most feared complication because of its associated significant morbidity and even mortality. Misidentification of the extrahepatic bile duct anatomy during the laparoscopic cholecystectomy seems to be the main cause of bile duct injury. ${ }^{3}$

Another example is iatrogenic injury of the ureter during colorectal or gynecologic surgery. In order to prevent such injury during procedures in which the ureter is specifically at risk, time is spent to identify the exact location of the ureter. A third example is inadvertent resection of parathyroid tissue during thyroid surgery. Preservation of the parathyroid glands during thyroid surgery is important to maintain calcium homeostasis. These parathyroid glands can be very hard to identify, causing unwilling resection of the parathyroid tissue, potentially resulting in hypoparathyroidism, which occurs in $18 \%$ of thyroidectomy patients. ${ }^{4}$

Hence in various procedures it is of great importance to be able to distinguish vital structures in the surgical field such as bile ducts, parathyroid glands, ureters and arteries. An optical tool that can help identify anatomy might facilitate the surgeon in critical intraoperative decision-making, thereby improving the safety and outcome for the patients.

One potential optical tool is near-infrared fluorescence (NIRF) imaging. The use of NIRF imaging is based on the administration of an exogenous contrast agent (i.e. a fluorescent dye), which can be visualized in the human body using a dedicated imaging system. The dye can be excited when exposed to a specific wave length in the near-infrared light spectrum, see Figure 1. When excited, the dye becomes fluorescent and can then be visualized and detected by using a special filter in front of the camera.

In Figure 2 a schematic overview of the use of this technique in practice is given. First, a fluorescent dye is administered, mostly intravenously. Then, a laser controller shines light in the exact right wavelength on this fluorescent dye, making it fluorescent. The fluorescent signal can then be detected using a camera which is equipped with a specific filter, only allowing the wavelength the fluorescent dye produces. The video-recordings can then be seen on the screen, with illuminating caused by the fluorescent dye in the patient's body. When the dye is located in the vascular system, fluorescence angiography can be performed using this technique. This opens the possibility to visualize perfusion of the 


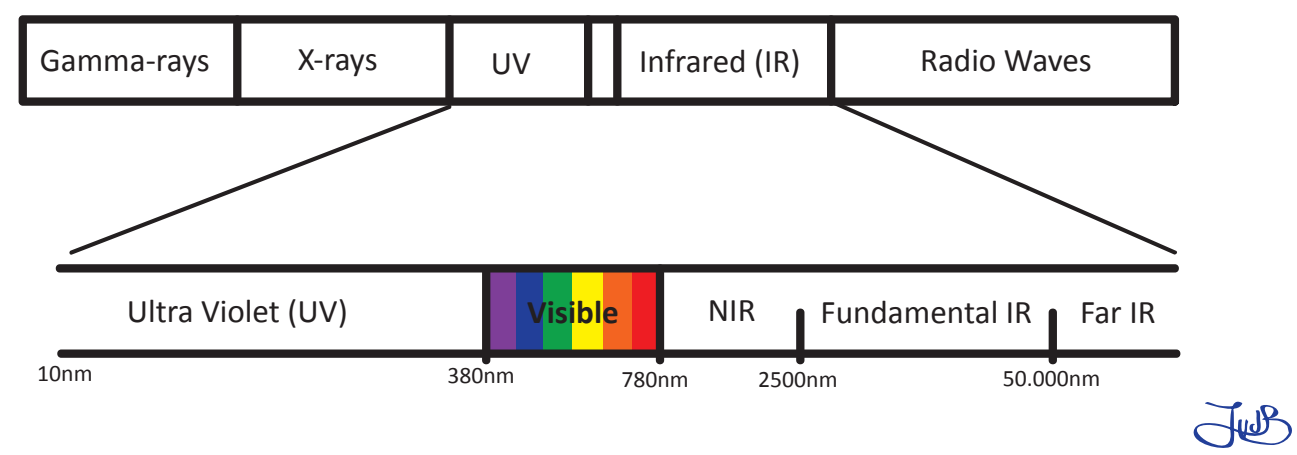

Figure 1 Wave length of visible and near infrared light

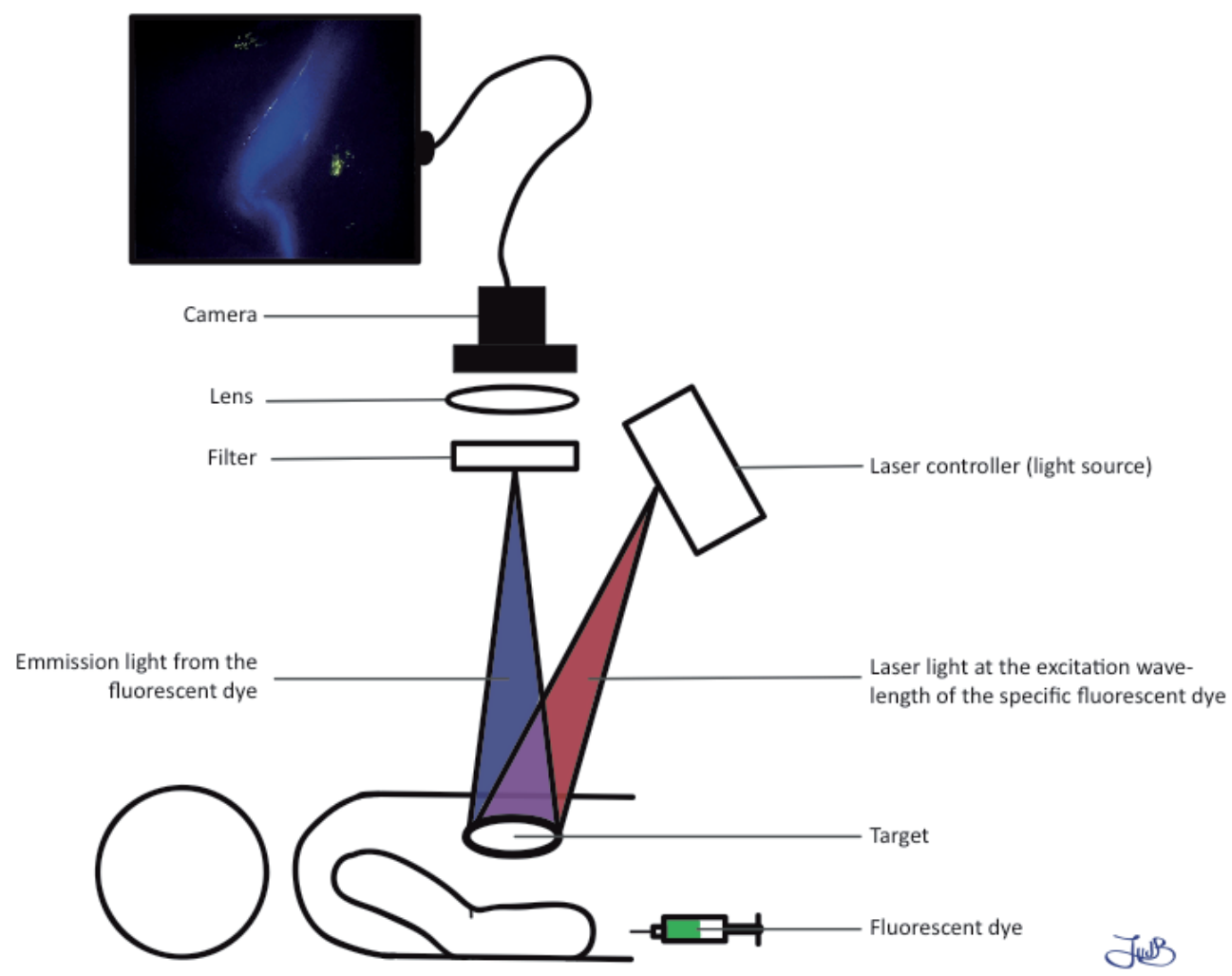

Figure 2 Schematic overview of the use of NIRF in surgery

intestines e.g. before and after constructing bowel anastomoses in colorectal surgery. When the dye is cleared by the liver, it will be collected in the bile ducts, facilitating the visualization of the extra-hepatic biliary anatomy. When cleared by the kidneys, the dye can be applied for ureteral delineation during pelvic interventions, e.g. in gynecologic, urologic or gastrointestinal surgery. 
The great potential of NIRF imaging is that the fluorescent dye can shine through a bile duct wall or vascular walls, because of a higher penetration depth (up to 5-10 $\mathrm{mm}$ ) compared to light in the visible light spectrum. Another advantage is that no radiological support is needed, which is for example mandatory in conventional x-ray cholangiography. Moreover, the patient is not exposed to any ionizing radiation.

Nowadays, several fluorescent dyes are used. The most commonly used dye, approved for clinical use, is indocyanine green (ICG). The use of ICG is reported since 1960 for assessing hepatic blood flow. ${ }^{5}$ In 1969, the use of infrared angiography was first reported to study pial vessels in dogs. ${ }^{6}$ In 1972, the first use of this technique in humans was described. ${ }^{7}$ Later, in 1973, Flower and Hochheimer actually report to have performed ICG-fluorescence angiography to visualize the choroidal vessels using a fundus camera with filters, showing only the exited light from the vessels filled with ICG and blocking the reflection in the fundus. ${ }^{8}$ This application is used to assess the choroidal vessels and thereby assess possible pathological choroidal patterns such as in senile macular degeneration, diabetic retinopathy and choroidal tumors. ${ }^{9}$

ICG binds quickly to albumin after intravenous administration. ${ }^{5}$ As mentioned, ICG is exclusively cleared by the liver. ICG is a small molecule (molecule mass 775 Dalton) and the plasma-half-life is short with 1-2 minutes. ${ }^{10}$ The excitation and emission peaks are respectively near 780 and $830 \mathrm{~nm}^{11}$

Another fluorescent dye approved for human use is methylene blue. Apart from the fluorescence, methylene blue is used for its pharmacological properties and as a staining agent. The pharmacological properties are used for treatment of methemoglobinemia' ${ }^{12}$, treatment of resistant plaque psoriasis ${ }^{13}$ and as antimalarial medication..$^{14}$ In very high doses it can be used as a vasopressor. ${ }^{15}$ Methylene blue is cleared by the kidneys and can therefore possibly be used to visualize the ureters. The peak excitation of methylene blue is $668 \mathrm{~nm}$, and peak emission at $688 \mathrm{~nm} .^{16}$

New NIRF dyes are being developed but are still at an experimental stage. The IR-Dyes (LICOR, Licon, Nebraska) are such agents. FDA approval for in-human use is expected to be obtained soon for the dyes IRDye ${ }^{\oplus} 800 \mathrm{CW}$, IRDye ${ }^{\oplus} 800$ BK $^{\text {and IRDye }}{ }^{\oplus} 800 \mathrm{NOS}^{17}{ }^{17}$ The excitation and emission peak of these dyes are 775 and 796 nm for IRDYE ${ }^{\oplus} 800 N^{2}$, 774 and $790 \mathrm{~nm}$ for IRDye ${ }^{\oplus} 800$ BK and 767 and $786 \mathrm{~nm}$ in IRDye ${ }^{\oplus} 800$ NOS. The advantages of these dyes are the similar wavelengths as compared to ICG, enabling the use of the presently available commercial systems and its improved hydrophilicity, causing faster biliary secretion and thereby earlier illumination of the bile ducts. In addition, these dyes are cleared both by the liver and the kidneys, making visualization of both the biliary anatomy as the ureters possible. 
As described, the NIRF imaging technique is not entirely new, as it has already been used for almost fifty years in ophthalmology. In surgery, this technique was first used in humans by Mitsuhashi in 2008 , followed by Ishizawa et al in 2010, both to visualize the biliary anatomy. ${ }^{18,19}$ In 2012, the first publication on NIRF imaging during laparoscopic cholecystectomy from our research group was published online. ${ }^{20}$ Hereafter, many more publications from all over the world followed. Other applications were described, such as the use of fluorescence imaging for assessment of bowel perfusion in anastomotic surgery ${ }^{21}$, intraoperative ureteral visualization $^{22}$ and real-time parathyroid gland identification. ${ }^{23}$ In these studies, almost exclusively promising results were found. However, the use of NIRF imaging was not yet implemented in standard clinical practice at the start of this thesis.

\section{AIMS AND OUTLINE OF THIS THESIS}

The overall aim of this thesis was to investigate some of the presently possible applications of near infrared fluorescence imaging during surgery in order to prevent iatrogenic damage due to misrecognition of the anatomy.

\section{PART I NEAR INFRARED FLUORESCENCE CHOLANGIOGRAPHY}

In this part, the use of near infrared fluorescence imaging in visualizing the biliary structures during laparoscopic cholecystectomy is described. Chapter $\mathbf{2}$ contains the protocol of an international multicenter international randomized controlled trial to investigate the added value of the use of near infrared fluorescence in laparoscopic cholecystectomies. Chapter $\mathbf{3}$ presents possible parameters of influence on the obtained intensity of the fluorescence signal during laparoscopic cholecystectomies. In Chapter 4, testing of two new fluorescent dyes for the visualization of the cystic duct and cystic artery is described. In Chapter 5, the objective measurement of fluorescence intensity in current literature is critically evaluated.

\section{PART II NEAR INFRARED FLUORESCENCE IMAGING IN COLORECTAL SURGERY}

In this part, the use of near infra-red fluorescence imaging in visualizing both the bowel perfusion and the ureter during colorectal surgery is described. Chapter 6 presents a systematic review investigating the added value of near infrared fluorescence angiography in anastomotic colorectal surgery. In Chapter 7, we present a clinical study to investigate the relation between fluorescence intensity of the bowel and postoperative inflammatory markers characterizing anastomotic leakage in order to explore the value of the intensity of the signal in predicting anastomotic healing. In Chapter $\mathbf{8}$ the use of Methylene Blue as a fluorescent dye for visualization of the ureter during colorectal surgery is evaluated. In 
Chapter 9, two new fluorescent dyes for ureter visualization are tested. In Chapter 10, a search for the optimal dose to gain the best fluorescent signal using a new fluorescent dye to visualize the ureters is presented.

\section{PART III NEAR INFRARED FLUORESCENCE IMAGING IN THYROID SURGERY}

Chapter 11 describes the use of near infrared fluorescence imaging in 30 thyroid surgeries to identify and assess remaining perfusion of the parathyroid glands. 


\section{REFERENCES}

1. Anderson O, Davis R, Hanna GB, Vincent CA. Surgical adverse events: a systematic review. American journal of surgery. 2013;206(2):253-62.

2. Healey MA, Shackford SR, Osler TM, Rogers FB, Burns E. Complications in surgical patients. Archives of surgery (Chicago, III : 1960). 2002;137(5):611-7; discussion 7-8.

3. Way LW, Stewart L, Gantert W, Liu K, Lee CM, Whang K, et al. Causes and prevention of laparoscopic bile duct injuries: analysis of 252 cases from a human factors and cognitive psychology perspective. Annals of surgery. 2003;237(4):460-9.

4. Ritter K, Elfenbein D, Schneider DF, Chen H, Sippel RS. Hypoparathyroidism after total thyroidectomy: incidence and resolution. J Surg Res. 2015;197(2):348-53.

5. Cherrick GR, Stein SW, Leevy CM, Davidson CS. Indocyanine green: observations on its physical properties, plasma decay, and hepatic extraction. J Clin Invest. 1960;39:592-600.

6. Kogure K, David NJ, Yamanouchi U, Choromokos E. Infrared absorption angiography of the fundus circulation. Arch Ophthalmol. 1970;83(2):209-14.

7. Flower RW. Infrared absorption angiography of the choroid and some observations on the effects of high intraocular pressures. Am J Ophthalmol. 1972;74(4):600-14.

8. Flower RW, Hochheimer BF. A clinical technique and apparatus for simultaneous angiography of the separate retinal and choroidal circulations. Invest Ophthalmol. 1973;12(4):248-61.

9. Bischoff PM, Flower RW. Ten years experience with choroidal angiography using indocyanine green dye: a new routine examination or an epilogue? Doc Ophthalmol. 1985;60(3):235-91.

10. Frangioni JV. In vivo near-infrared fluorescence imaging. Curr Opin Chem Biol. 2003;7(5): 626-34.

11. Gurfinkel M, Thompson AB, Ralston W, Troy TL, Moore AL, Moore TA, et al. Pharmacokinetics of ICG and HPPH-car for the detection of normal and tumor tissue using fluorescence, nearinfrared reflectance imaging: a case study. Photochem Photobiol. 2000;72(1):94-102.

12. Wright RO, Lewander WJ, Woolf AD. Methemoglobinemia: etiology, pharmacology, and clinical management. Ann Emerg Med. 1999;34(5):646-56.

13. Salah M, Samy N, Fadel M. Methylene blue mediated photodynamic therapy for resistant plaque psoriasis. J Drugs Dermatol. 2009;8(1):42-9.

14. Schirmer RH, Adler H, Pickhardt M, Mandelkow E. "Lest we forget you--methylene blue...". Neurobiol Aging. 2011;32(12):2325 e7-16.

15. Pasin L, Umbrello M, Greco T, Zambon M, Pappalardo F, Crivellari M, et al. Methylene blue as a vasopressor: a meta-analysis of randomised trials. Crit Care Resusc. 2013;15(1):42-8.

16. Matsui A, Lee BT, Winer JH, Vooght CS, Laurence RG, Frangioni JV. Real-time intraoperative near-infrared fluorescence angiography for perforator identification and flap design. Plast Reconstr Surg. 2009;123(3):125e-7e.

17. Barnes TG, Volpi D, Cunningham C, Vojnovic B, Hompes R. Improved urethral fluorescence during low rectal surgery: a new dye and a new method. Tech Coloproctol. 2018;22(2):115-9.

18. Ishizawa T, Bandai Y, ljichi M, Kaneko J, Hasegawa K, Kokudo N. Fluorescent cholangiography illuminating the biliary tree during laparoscopic cholecystectomy. The British journal of surgery. 2010;97(9):1369-77.

19. Mitsuhashi N, Kimura F, Shimizu H, Imamaki M, Yoshidome H, Ohtsuka M, et al. Usefulness of intraoperative fluorescence imaging to evaluate local anatomy in hepatobiliary surgery. J Hepatobiliary Pancreat Surg. 2008;15(5):508-14.

20. Schols RM, Bouvy ND, Masclee AA, van Dam RM, Dejong CH, Stassen LP. Fluorescence cholangiography during laparoscopic cholecystectomy: a feasibility study on early biliary tract delineation. Surg Endosc. 2013;27(5):1530-6.

21. Kudszus S, Roesel C, Schachtrupp A, Hoer JJ. Intraoperative laser fluorescence angiography in colorectal surgery: a noninvasive analysis to reduce the rate of anastomotic leakage. Langenbeck's archives of surgery / Deutsche Gesellschaft fur Chirurgie. 2010;395(8):1025-30. 
22. Verbeek FP, van der Vorst JR, Schaafsma BE, Swijnenburg RJ, Gaarenstroom KN, Elzevier HW, et al. Intraoperative near infrared fluorescence guided identification of the ureters using low dose methylene blue: a first in human experience. J Urol. 2013;190(2):574-9.

23. Suh YJ, Choi JY, Chai YJ, Kwon H, Woo JW, Kim SJ, et al. Indocyanine green as a near-infrared fluorescent agent for identifying parathyroid glands during thyroid surgery in dogs. Surg Endosc. 2015;29(9):2811-7. 
PART I 
NEAR INFRARED FLUORESCENCE CHOLANGIOGRAPHY 
CHAPTER 2

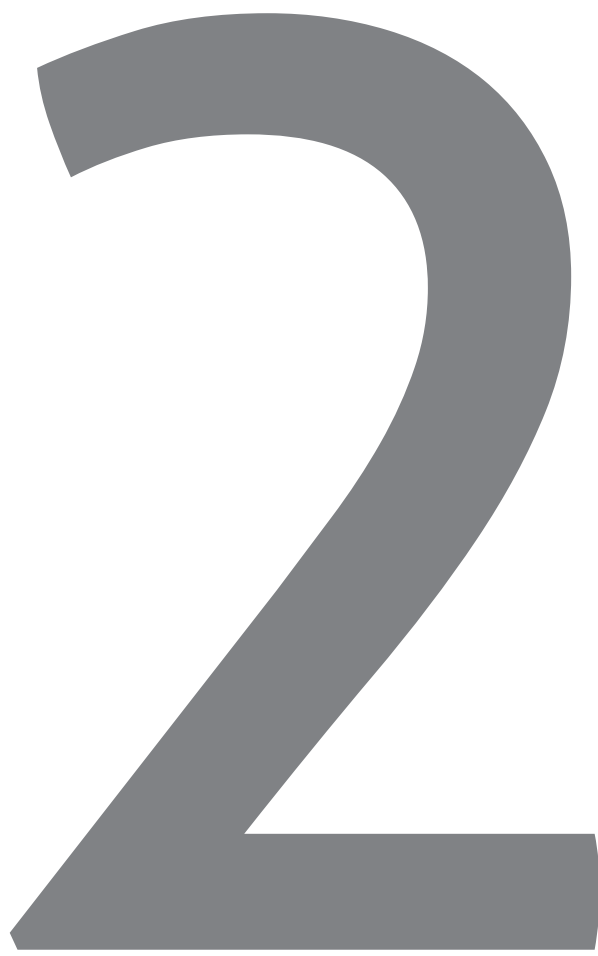




\section{Near-infrared fluorescence cholangiography assisted laparoscopic cholecystectomy versus conventional laparoscopic cholecystectomy (FALCON trial): study protocol for a multicenter randomized controlled trial}

Jacqueline van den Bos, Rutger M. Schols, Misha D. Luyer, Ronald M. van Dam, Alexander L. Vahrmeijer, Wilhelmus J. Meijerink, Paul D. Gobardhan, Gooitzen M. van Dam, Nicole D. Bouvy, Laurents P.S. Stassen.

BMJ Open. 2016;6(8):e011668. 


\section{ABSTRACT}

\section{Introduction}

Misidentification of the extrahepatic bile duct anatomy during laparoscopic cholecystectomy (LC) is the main cause of bile duct injury. Easier intraoperative recognition of the biliary anatomy may be accomplished by using near-infrared fluorescence (NIRF) imaging after an intravenous injection of indocyanine green (ICG). Promising results were reported for successful intraoperative identification of the extrahepatic bile ducts compared to conventional laparoscopic imaging. However, routine use of ICG fluorescence laparoscopy has not gained wide clinical acceptance yet due to a lack of high-quality clinical data. Therefore, this multicentre randomised clinical study was designed to assess the potential added value of the NIRF imaging technique during LC.

\section{Methods and Analysis}

A multicentre, randomized controlled clinical trial will be carried out to assess the use of NIRF imaging in LC. In total, 308 patients scheduled for an elective LC will be included. These patients will be randomised into a NIRF imaging laparoscopic cholecystectomy (NIRF-LC) group and a conventional laparoscopic cholecystectomy (CLC) group. The primary end point is time to 'critical view of safety' (CVS). Secondary end points are 'time to identification of the cystic duct (CD), of the common bile duct, the transition of $C D$ in the gallbladder and the transition of the cystic artery in the gallbladder, these all during dissection of CVS'; 'total surgical time'; 'intraoperative bile leakage from the gallbladder or cystic duct'; 'bile duct injury'; 'postoperative length of stay', 'complications due to the injected ICG'; 'conversion to open cholecystectomy'; 'postoperative complications (until 90 days postoperatively)' and 'cost-minimisation'.

\section{Ethics and dissemination}

The protocol has been approved by the Medical Ethical Committee of Maastricht University Medical Center/Maastricht University; the trial has been registered at ClinicalTrials.gov. The findings of this study will be disseminated widely through peer-reviewed publications and conference presentations. 


\section{INTRODUCTION}

Laparoscopic cholecystectomy (LC) is the most commonly performed laparoscopic procedure in the Netherlands, with almost 23000 procedures annually.' Bile duct injury during this procedure is rare with an incidence of $0.3-0.7 \% .^{2-5}$ However, when bile duct injury or vascular injury is present, it results in significant clinical relevant morbidity and mortality, lower quality of life and extra costs. ${ }^{6-10}$ Bile duct injury will generally lead to bile leakage and abdominal sepsis and can lead to bile duct obstruction with obstructive jaundice eventually leading to orthotropic liver transplantation or both.' Late recognition and management of bile duct injuries can lead to severe deterioration in the patient's condition, progressing to biliary peritonitis, sepsis, multiorgan failure and eventually death. Therefore, early recognition and treatment is important., ${ }^{71}$ Misidentification of the extrahepatic bile duct anatomy during LC is the main cause of bile duct injury. ${ }^{12}$

To reduce this risk of bile duct injury, the critical view of safety (CVS) technique was introduced by Strasberg in 1995. ${ }^{13}$ A recent Society of American Gastrointestinal and Endoscopic Surgeons (SAGES) expert Delphi consensus deemed the CVS as being the most important factor for overall safety, ${ }_{1}^{14}$ in accordance with the current Dutch Surgical Society Guideline for Laparoscopic Cholecystectomy. ${ }^{15}$ To establish CVS, two observation windows need to be created: one window between the cystic artery (CA), cystic duct (CD) and gallbladder and another between the CA, gallbladder and liver (see Figure 1). The CVS technique is especially aimed at mobilising the gallbladder neck from the liver, in order to obtain a circumferential identification of the transition of the $C D$ into the gallbladder. Nowadays, the CVS technique is the gold standard to perform a safe cholecystectomy with identification of the vital structures such as the CD. ${ }^{16-20}$

According to a Dutch nationwide survey in 2011, 97.6\% of the Dutch surgeons use the CVS technique. ${ }^{21}$ However, according to a recent study by Nijssen et al, ${ }^{22}$ only in $10 \%$ of the laparoscopic cholecystectomies CVS is actually established. This could mean that is more difficult to establish CVS than thought before, thus resulting in more bile duct injury than necessary.

Nowadays, there are several imaging techniques, such as intraoperative cholangiography (IOC) and near infrared fluorescence (NIRF) imaging, to identify the relevant anatomical structures easier. IOC has been advised to reduce the risk of bile duct injury. ${ }^{2,16,23}$

However, this radiological imaging of the biliary tree is not adopted worldwide in standard LC, as the procedure takes time, involves radiation exposure and requires additional equipment and manpower. Moreover, the interpretation of an intraoperative cholangiogram with potentially distorted anatomy clearly depends on the expertise of the surgeon. Therefore, worldwide consensus about implementation of IOC is still lacking. ${ }^{24}$

NIRF imaging after intravenous injection of indocyanine green (ICG) is a promising new technique for easier intraoperative recognition of the biliary anatomy. ${ }^{25,26}$ ICG is cleared 
quickly and exclusively by the liver after intravenous administration and has a very wellknown pharmacokinetic and safety profile. Neither radiological support nor additional intervention such as opening the cystic or common bile duct (CBD) is required, making it an easy, real-time and flexible technique to use during surgery. By real-time identification of the vital structures being the $C D$ and $C B D$ within the already adapted CVS technique, it may improve the outcome of LC. 16,27,28 NIRF imaging using ICG has been evaluated in various animal models ${ }^{29-31}$ and in open, laparoscopic and single-incision laparoscopic cholecystectomies. ${ }^{30,}$ 32-34 Promising results were presented for safe and successful intraoperative identification of the $C B D$ and the $C D$, compared to conventional laparoscopic imaging. Furthermore, a clinical study $(n=30)$ showed that the NIRF imaging technique provided significantly earlier identification of the extrahepatic bile ducts during the CVS dissection phase: up to $10 \mathrm{~min}$ earlier identification of the CD and CBD could be obtained. ${ }^{35}$ Real-time imaging of the hepatic and cystic arteries was also achieved when a repeated dose of ICG was given. ${ }^{35-37}$

Despite these encouraging results derived from clinical feasibility studies, the routine use of ICG fluorescence laparoscopy has not gained wide clinical acceptance yet due to a lack of high-quality clinical data.

Therefore, a multicentre randomised clinical study was designed to assess the added value of the NIRF imaging technique during LC. The ultimate goal of this technique is to perform a safer procedure leading to a reduction in vascular and bile duct injuries. The primary objective of the present study is to evaluate whether earlier establishment of CVS can be obtained using the NIRF imaging technique during LC.

\section{METHODS AND ANALYSIS}

\section{Primary aim}

The main objective of the study is to evaluate whether earlier establishment of the CVS can be obtained using the NIRF imaging technique during elective LC, by applying NIRF imaging as an adjunct to conventional laparoscopic imaging versus conventional laparoscopic imaging alone.

\section{Hypothesis}

It is hypothesised that standard application of NIRF imaging during LC will result in establishment of CVS at least 5 min earlier and with more certainty regarding visualisation of biliary anatomy when compared to conventional laparoscopic imaging alone.

\section{Study design}

This multicentre randomised controlled clinical trial includes two randomisation arms: a NIRF-LC (laparoscopic cholecystectomy) group - this group of patients will undergo NIRF 

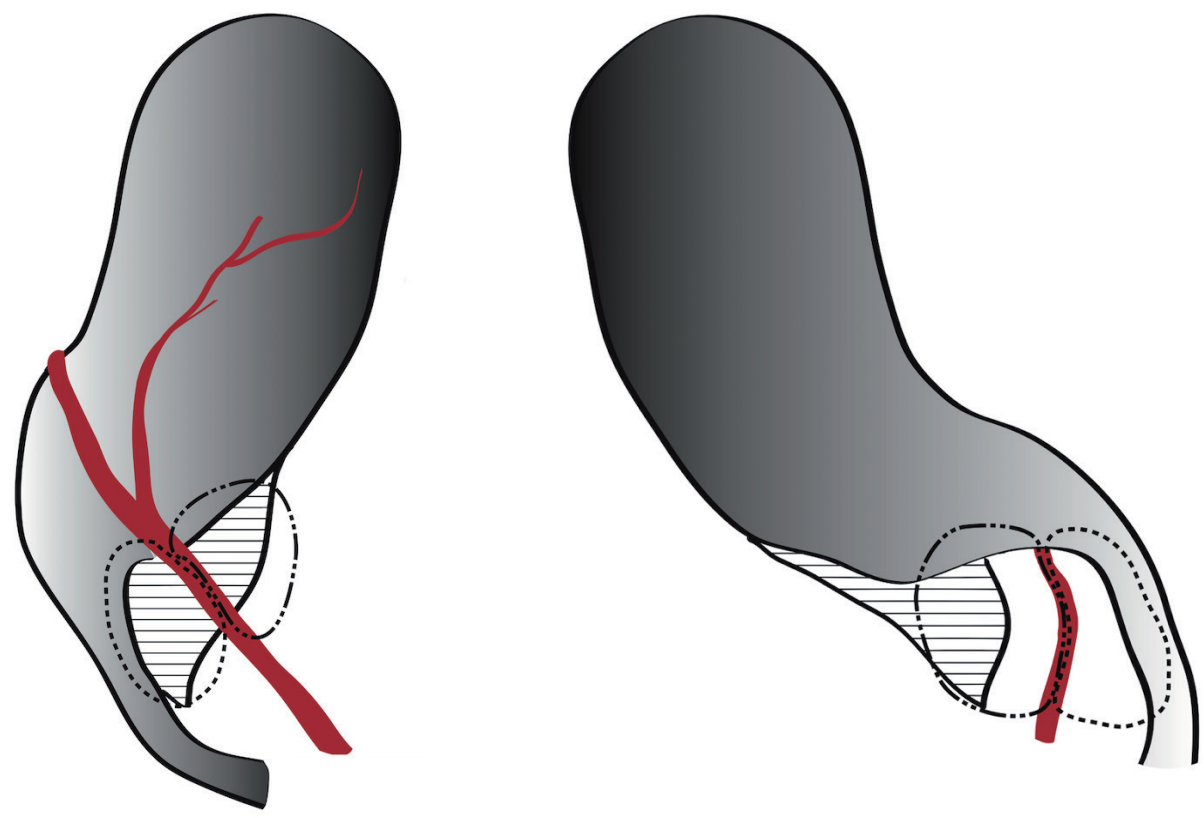

Window of CVS between cystic artery, cystic duct and gallbladder

-..-.-. Window of CVS between cystic artery, gallbladder and liver

Gallbladder bed where detached from liver

Figure 1a Anterior view of CVS

Figure 1b Posterior view of CVS

cholangiography assisted laparoscopic cholecystectomy and a conventional laparoscopic cholecystectomy (CLC) control group-this group will undergo CLC.

\section{Setting}

In the FALCON trial, a total of 308 patients will be included at the Departments of Surgery of the participating centres. The centres will be supported by the trial coordinator (JvdB) and by the Clinical Trial Center Maastricht (CTCM) (see also under the 'data monitoring' section). Further, no additional strategies for achieving adequate participant enrolment to reach the target sample size are considered necessary, as LC is a commonly performed surgery.

\section{Sample size calculation}

The number of 308 participants is based on pilot data, ${ }^{35,}{ }^{38}$ where the identification of the $C D$ and $C B D$ was established, respectively, 11 and 10 min earlier using fluorescence laparoscopic imaging compared to conventional laparoscopic imaging. A sample size of 131 for each randomisation arm has been calculated to detect a reduction in 'time to establishment of CVS' of at least 5 min with a power of $80 \%$ and an a of $0.05(95 \% \mathrm{Cl})$. Assuming a withdrawal rate of $15 \%$ (due to usual reasons for dropout in combination with 
technical difficulties concerning the video recordings) during the trial, we will require a total of $308(n=2 \times 131+15 \%)$.

All patients (age $>18$ years) scheduled for an elective LC and meeting the inclusion criteria will be suitable for inclusion.

\section{Inclusion criteria}

The inclusion criteria are as follows: male and female patients, aged 18 years and above, scheduled for elective LC, with normal liver and renal function, no hypersensitivity for iodine or ICG, able to understand the nature of the study procedures, willing to participate and give written informed consent and Physical Status Classification of ASA I/ASA II.

\section{Exclusion criteria}

The exclusion criteria are as follows: age < 18 years, liver or renal insufficiency, known iodine or ICG hypersensitivity, pregnancy or breastfeeding, not able to understand the nature of the study procedure and a Physical Status Classification of ASA III and above.

Participants can leave the study at any time for any reason if they wish to do so without any consequences.

The investigator can decide to withdraw a participant from the study for urgent medical reasons. Conversion to open cholecystectomy, before CVS is established, is a reason for study withdrawal. Furthermore, if the video recordings of the laparoscopic procedure were not successful, the procedure will be unsuitable for analysis of all predefined end points. There are no other specific criteria for withdrawal. In case of withdrawal, participants will be replaced to achieve the calculated sample size. All inclusions will be analysed on an intention-to-treat basis.

\section{Randomisation}

All included patients will be randomised centrally using block randomisation with sealed envelopes and stratification per participating centre. After signing the informed consent form, the next sealed envelope in line will be opened by the coordinating investigator. There will be no blinding of patients or surgeons.

\section{Intervention}

The CLC group will undergo conventional laparoscopic cholecystectomy. The NIRF-LC group will undergo near infrared fluorescence cholangiography using a laparoscopic NIRF imaging system (Karl Storz GmbH, Tuttlingen, Germany). To obtain fluorescence imaging of the biliary tract and CA, a NIRF contrast agent will be administered. Directly after the induction of anaesthesia, $2.5 \mathrm{mg}$ of ICG $(2.5 \mathrm{mg} / \mathrm{mL}$; Diagnostic Green, Aschheim, Germany) will be given intravenously. A repeat injection of $2.5 \mathrm{mg}$ will be administered for concomitant arterial and biliary fluorescence delineation after achievement of CVS. 


\section{Outcome measures}

The primary outcome measure is time to identification of CVS. This end point is used as a surrogate for bile duct identification without surgical exploration. CVS is established if the following three criteria are met:

1. Mobilisation of the gallbladder infundibulum for one-third of the length of the gallbladder from the liver bed.

2. Circumferential exposure of the $C D$ and confirmation of its transition in the gallbladder.

3. Circumferential exposure of the $C A$ and confirmation of its transition in the gallbladder.

Secondary outcome measures are listed in Table 1.

\section{Data collection}

Intraoperatively, a case report form will be filled in. A structure is scored as 'identified' if its localisation is confirmed with great certainty by the experienced surgeon. The attending surgeon will be consulted to decide whether he believes CVS is established.

In accordance with regular care, all laparoscopic surgical procedures will be digitally recorded. An expert panel, consisting of three highly experienced laparoscopic surgeons, will analyse the data using video recordings: time until identification of the $C D$ and of its transition into the gallbladder; time until identification of the CA and its transition into the gallbladder during dissection of CVS and when and whether CVS is established. Eventually, all five observers (the surgeon or surgical trainee, $\mathrm{PhD}$ researcher or local researcher during the operation and the three postoperative observers) will individually assess the abovementioned end points. The mean values of these five assessments will be used for each of the end points. All clinical data are prospectively registered in a database.

OsiriX V.5.5.1. Imaging Software (Prixmeo, Geneva, Switzerland) will be used for objective assessment of the degree of fluorescence illumination in the extrahepatic bile ducts. The fluorescence images will be analysed by determining the target-to-background ratio (TBR). TBR is defined as the mean fluorescence intensity (FI) of two-point regions of interest (ROIs) in the target (ie, CBD, CD or CA) minus the mean Fl of two background (BG) ROls in the liver hilum, divided by the mean FI of the two background ROIs in the liver hilum; that is TBR $=(\mathrm{FI}$ of target-FI of BG)/FI of BG.

The costs made in the two groups will be compared, resulting in a cost-minimisation analysis. This analysis will include the costs made by using the operation theatre in terms of fluorescence laparoscopy equipment, the fluorescent dye ICG, morbidity, mortality and postoperative hospital stay.

In Figure 2, flow charts of the study procedure for the CLC-group and the NIRF-LC group are presented. 
Table 1 Secondary outcome measures

\begin{tabular}{|c|c|}
\hline Outcome measure & Definition \\
\hline Time until identification of the cystic duct (CD) & Time in minutes \\
\hline Time until identification of common bile duct & Time in minutes \\
\hline $\begin{array}{l}\text { Time until identification of the transition of CD } \\
\text { into the gallbladder }\end{array}$ & Time in minutes \\
\hline $\begin{array}{l}\text { Time until identification of the transition of the } \\
\text { cystic artery (CA) into the gallbladder }\end{array}$ & Time in minutes \\
\hline Total Surgical time & $\begin{array}{l}\text { Time in minutes from skin incision to the end of } \\
\text { skin closure }\end{array}$ \\
\hline $\begin{array}{l}\text { Visualization of CVS and visualization of the } \\
\text { transition of the cystic duct and cystic artery into } \\
\text { the gallbladder }\end{array}$ & Time in minutes \\
\hline $\begin{array}{l}\text { Intraoperative bile leakage from the gallbladder } \\
\text { or cystic duct }\end{array}$ & Visualized bile leakage or spill during surgery. \\
\hline Bile duct injury & $\begin{array}{l}\text { Any injury to the main biliary tree; will be } \\
\text { classified using the Strasberg Classification } \\
\text { System } \\
\text { Type A: Injury to the cystic duct or from minor } \\
\text { hepatic ducts draining the liver bed. } \\
\text { Type B: Occlusion of biliary tree, commonly } \\
\text { aberrant right hepatic duct(s). } \\
\text { Type C: Transection without ligation of aberrant } \\
\text { right hepatic duct(s). } \\
\text { Type D: Lateral injury to a major bile duct. } \\
\text { Type E: Injury to the main hepatic duct; classified } \\
\text { according to level of injury. }\end{array}$ \\
\hline Postoperative length of hospital stay & $\begin{array}{l}\text { Duration from date of admission (included) to } \\
\text { date of discharge (included) }\end{array}$ \\
\hline Complications due to injected contrast agent & $\begin{array}{l}\text { Any complication potentially caused by injected } \\
\text { ICG }\end{array}$ \\
\hline Conversion to open cholecystectomy & $\begin{array}{l}\text { Laparoscopic approach converted to an } \\
\text { open operation, or in which an abdominal } \\
\text { incision to assist the procedure was needed. }\end{array}$ \\
\hline 90-day all-cause postoperative complications & $\begin{array}{l}\text { Any complication, up to } 90 \text { days, described by } \\
\text { the Clavien-Dindo classification of postoperative } \\
\text { complications } \\
\text { Specific attention to bile leak, CBD injury, wound } \\
\text { infection, intra-abdominal collection, pancreatitis, } \\
\text { CBD stones, ICU/HDU readmissions; prospectively } \\
\text { assessed during admission; thereafter } \\
\text { immediately to be reported to study coordinator }\end{array}$ \\
\hline Cost Minimization & $\begin{array}{l}\text { Difference in costs (in Euros) between } \\
\text { conventional LC and NIRF LC }\end{array}$ \\
\hline
\end{tabular}

CA: cystic artery; CBD: common bile duct; CD: cystic duct; CVS: critical view of safety; LC: laparoscopic cholecystectomy; NIRF: nearinfrared fluorescence.

\section{Data validation and management}

Patient data will be anonymously registered and analysed by comparing NIRF-LC with CLC. Only the investigators will have access to the patient data after informed consent is given. 


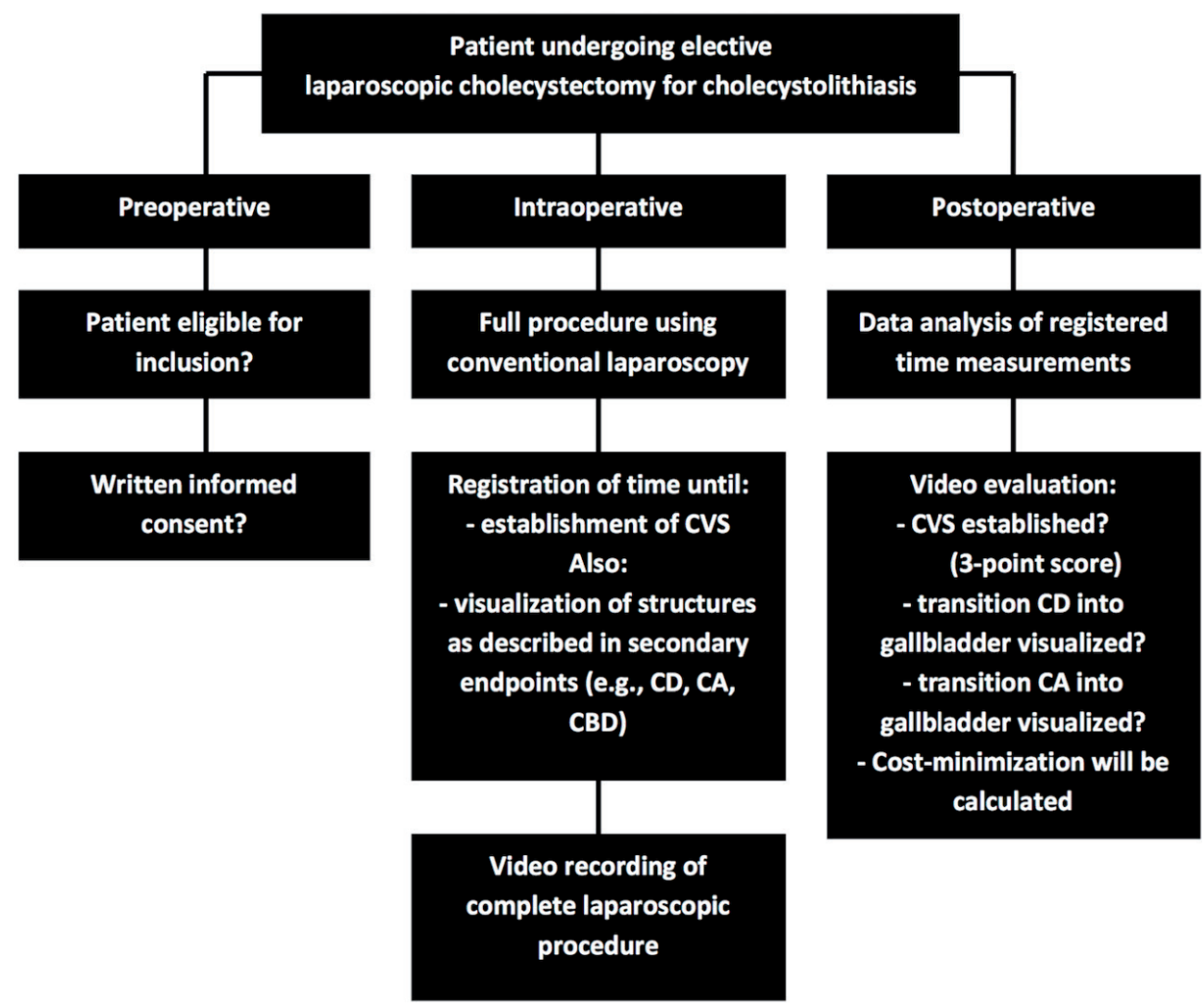

Figure 2a Flow chart of study procedures: conventional laparoscopic cholecystectomy (CLC) group

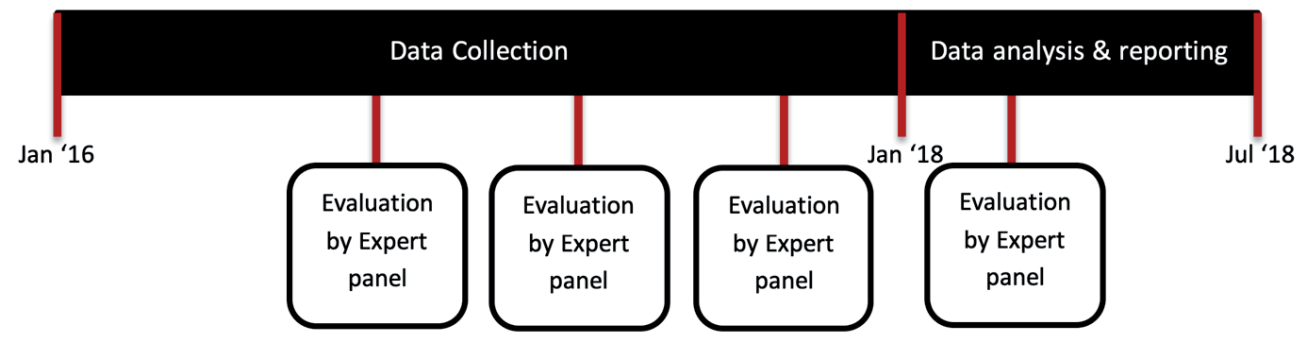

Figure 3 Study timeline

\section{Study timeline}

In Figure 3, the study timeline is presented. From January 2016 until January 2018, data will be collected; in September 2016, March 2017, September 2017 and March 2018, the expert panel will evaluate the video material for end points; around July 2018, data analysis is expected to be complete.

Participants will be informed about the study during their preoperative visit to the outpatient clinic. Thereafter, patients have at least a week to consider participation in the 


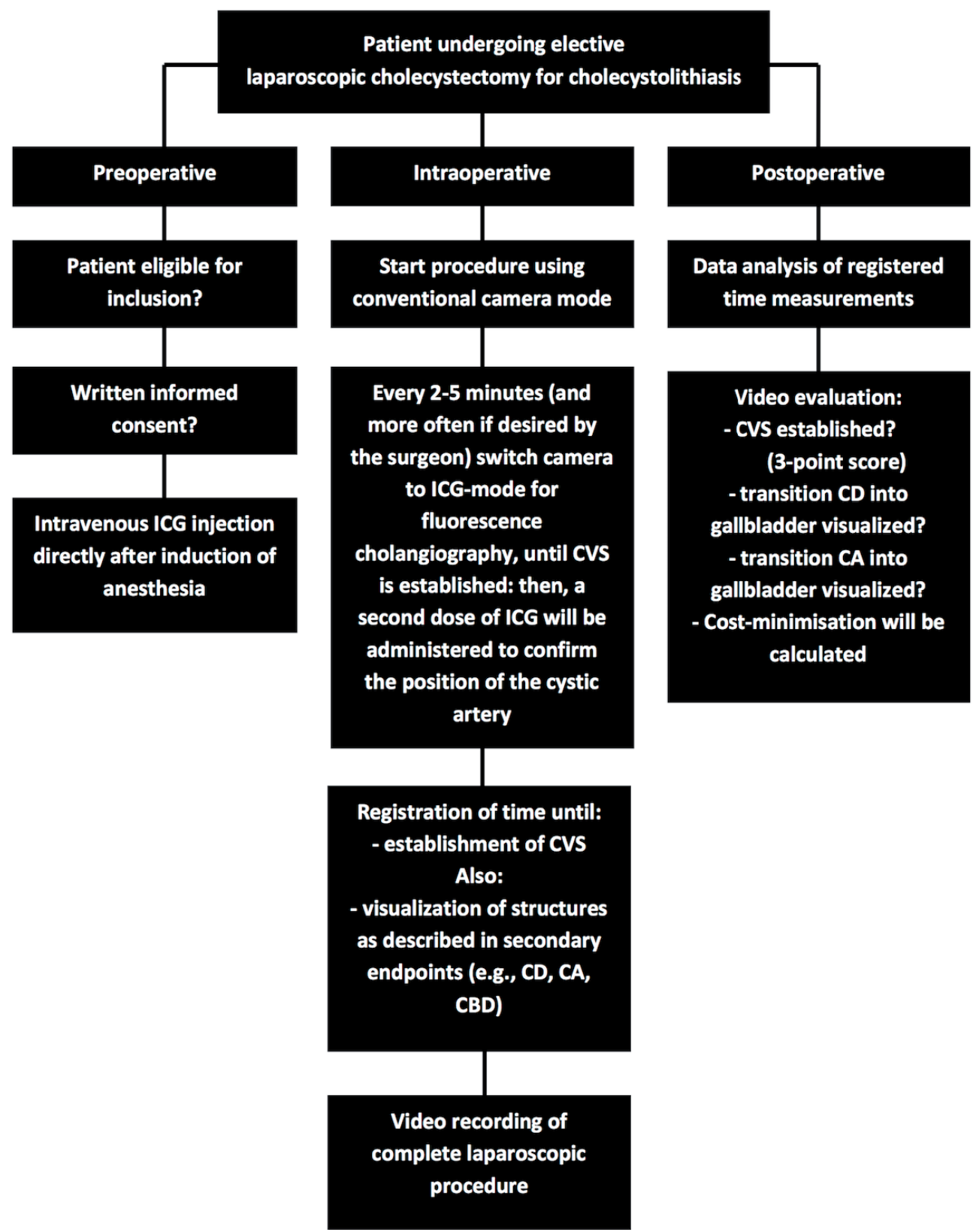

Figure 2b Flow chart of study procedures: NIRF laparoscopic cholecystectomy (NIRF-LC) group

study. During their elective surgery, the near-infrared fluorescence laparoscopy will be used if the patient is randomised in the NIRF-LC group. After surgery, a 90-day follow-up period follows, and then possible complications will be evaluated. 


\section{Statistical analysis}

For statistical analysis, the most recent version of SPSS (IBM, Armonk, New York, USA) will be used. Baseline characteristics such as patient clinical history (including previous surgery), age, body mass index and indication for the procedure will be recorded and compared between the intervention (NIRF-LC) and control groups (CLC). Categorical baseline variables will be compared using a $x^{2}$ test, while numerical variables will be compared by the independent sample t-test or the Mann-Whitney $U$ test, depending on the distribution.

The primary outcome measure, namely, time until establishment of CVS, will be given in minutes, with a mean and SD. A linear regression analysis will be applied for determination of possible significant differences between the time measurements, therewith comparing the NIRF-LC group to the CLC group. This will be conducted to determine whether a reduction in time can in fact be achieved using the NIRF imaging technique compared to CLC.

All numerical secondary outcomes such as time until visualisation of CD and CA will be analysed using a linear regression model. In case of missing values, a Cox regression analysis will be performed. Missing values can occur especially in the postoperative analysis by the expert panel, when the panel concludes that, contrary to the opinion of the operating team, actually no CVS was obtained or that the transition of the CD or CA in the gallbladder had actually not been properly identified. All categorical secondary outcomes such as bile duct injury and conversion to open surgery will be analysed with a logistic regression model.

\section{Data monitoring}

An independent data monitoring committee will monitor the study procedures and data management. This team consists of independent and certified persons from the CTCM. No interim analysis will be performed. Adverse events and serious adverse events will be centrally reported in the online database, toetsingonline.nl.

\section{ETHICS AND DISSEMINATION}

The proposed study is approved by the Medical Ethics committee of Maastricht University Medical

Center/Maastricht University. Possible protocol amendments will be sent to the Medical Ethics Committee of Maastricht University Medical Center/Maastricht University. After approval, the changes will be communicated on clinicaltrials.gov and to the relevant parties.

Is there scientific and clinical value in conducting this study?

Despite the promising results from previous feasibility studies, a lack of solid clinical data precludes wide clinical acceptance of the routine use of ICG fluorescence laparoscopy. This multicentre randomised clinical study can provide such data. 


\section{Risk-benefit assessment}

There are no additional risks accompanied by the laparoscopic NIRF imaging systems, compared to conventional laparoscopic imaging. The gifts of ICG are the only additional (minimally) invasive interventions for the patient. ICG preparations can, in very rare cases, cause nausea and anaphylactoid or anaphylactic reactions $(<1: 10000)$. Patients with terminal renal insufficiency seem to be more prone for such an anaphylactic reaction. Estimated death due to anaphylaxis is reported as $<1$ per $330000 .{ }^{40-43}$ Symptoms include anxiety, feeling of warmth, pruritus, urticaria, acceleration of heart rate, decrease in blood pressure, shortness of breath, bronchospasm, flushing, cardiac arrest, laryngospasm, facial oedema and nausea. Together with the anaphylactoid reaction, hyper-eosinophilia may occur. If, contrary to expectations, symptoms of anaphylaxis occur, the following measures will be taken: stop further administration of ICG, leave injection catheter or cannula in the vein, keep airways free, inject 100-300 mg hydrocortisone or a similar preparation by rapid intravenous injection, substitute volume with isotonic electrolyte solution, give oxygen and monitor the circulation and slowly administer antihistamines intravenously. In case of an anaphylactic shock, the patient will be placed in the recumbent position with legs raised, volume will be rapidly substituted with, for example, isotonic electrolyte solution (pressure infusion), plasma expanders. Furthermore, 0.1-0.5 mg epinephrine will be administered and immediately diluted to $10 \mathrm{~mL}$ with $0.9 \%$ saline intravenously. If necessary, this will be repeated after $10 \mathrm{~min}$.

The benefit for the patients in the NIRF-LC group will possibly include a shorter period to the establishment of CVS and the clearer identification of CVS and its anatomical components.

\section{Do the individuals give informed consent?}

To each patient, that is, a potential candidate for inclusion, thorough patient information will be given. From each individual who is willing to participate, written informed consent will be obtained by one of the investigators.

The ethical issues of the trial will be thoroughly explained and discussed, verbally and in writing. The basic principles laid down in the Declaration of Helsinki ${ }^{44}$ will be followed throughout the execution of the trial. Accordingly, each participant has the right to withdraw from the study at any given moment without having to explain this decision in any way. 


\section{REFERENCES}

1. Statistiek CBvd. Operaties in het ziekenhuis; soort opname, leeftijd en geslacht, 199520102010 [updated 05-02-2014]. http://statline.cbs.nl/StatWeb/publication/?VW=T\&DM= $\mathrm{SLNL} \& \mathrm{PA}=80386 \mathrm{NED} \& \mathrm{LA}=\mathrm{NL}$

2. Flum DR, Dellinger EP, Cheadle $A$, et al. Intraoperative cholangiography and risk of common bile duct injury during cholecystectomy. JAMA 2003;289:1639-44.

3. Fletcher DR, Hobbs MS, Tan P, et al. Complications of cholecystectomy: risks of the laparoscopic approach and protective effects of operative cholangiography: a population-based study. Ann Surg 1999;229:449-57.

4. Nuzzo G, Giuliante F, Giovannini I, et al. Bile duct injury during laparoscopic cholecystectomy: results of an Italian national survey on 56591 cholecystectomies. Arch Surg 2005;140:986-92.

5. Waage A, Nilsson M. latrogenic bile duct injury: a population-based study of 152776 cholecystectomies in the Swedish Inpatient Registry. Arch Surg 2006;141:1207-13.

6. Bobkiewicz A, Krokowicz $Ł$, Banasiewicz T, et al. latrogenic bile duct injury. A significant surgical problem. Assessment of treatment outcomes in the department's own material. Pol Przegl Chir 2014;86:576-83.

7. Booij KA, de Reuver PR, Yap K, et al. Morbidity and mortality after minor bile duct injury following laparoscopic cholecystectomy. Endoscopy 2015;47:40-6.

8. Dolan JP, Diggs BS, Sheppard BC, et al. Ten-year trend in the National volume of bile duct injuries requiring operative repair. Surg Endosc 2005;19:967-73.

9. Boerma D, Rauws EA, Keulemans YC, et al. Impaired quality of life 5 years after bile duct injury during laparoscopic cholecystectomy: a prospective analysis. Ann Surg 2001;234:750-7.

10. Landman MP, Feurer ID, Moore DE, et al. The long-term effect of bile duct injuries on healthrelated quality of life: a meta-analysis. HPB (Oxford) 2013;15:252-9.

11. Törnqvist B, Strömberg C, Persson G, et al. Effect of intended intraoperative cholangiography and early detection of bile duct injury on survival after cholecystectomy: population based cohort study. BMJ 2012;345:e6457.

12. Way LW, Stewart L, Gantert W, et al. Causes and prevention of laparoscopic bile duct injuries: analysis of 252 cases from a human factors and cognitive psychology perspective. Ann Surg 2003;237:460-9.

13. Strasberg SM, Hertl M, Soper NJ. An analysis of the problem of biliary injury during laparoscopic cholecystectomy. J Am Coll Surg 1995;180:101-25.

14. Pucher PH, Brunt LM, Fanelli RD, et al. SAGES expert Delphi consensus: critical factors for safe surgical practice in laparoscopic cholecystectomy. Surg Endosc 2015;29:3074-85.

15. Lange JF SL. Best practice: De techniek van de laparoscopische cholecystectomie (Critical View of Safety [CVS]; Werkgroep Endoscopische Chirurgie van de Nederlandse Vereniging voor Heelkunde). 2006. http://www.nvgic.nl/richtlijnen/Best\%20Practice\%20Laparoscopische\%20 Cholecystectomie.pdf

16. Buddingh KT, Nieuwenhuijs VB, van Buuren L, et al. Intraoperative assessment of biliary anatomy for prevention of bile duct injury: a review of current and future patient safety interventions. Surg Endosc 2011;25:2449-61.

17. Dziodzio $T$, Weiss $S$, Sucher $R$, et al. A 'critical view' on a classical pitfall in laparoscopic cholecystectomy! Int J Surg Case Rep 2014;5:1218-21.

18. Kaczynski J, Hilton J. A gallbladder with the "hidden cystic duct": a brief overview of various surgical techniques of the Calot's triangle dissection. Interv Med Appl Sci 2015;7:42-5.

19. Strasberg SM, Brunt LM. Rationale and use of the critical view of safety in laparoscopic cholecystectomy. J AmCollSurg 2010;211:132-8.

20. Vettoretto N, Saronni C, Harbi A, et al. Critical view of safety during laparoscopic cholecystectomy. JSLS 2011;15:322-5. 
21. Buddingh KT, Hofker HS, ten Cate Hoedemaker HO, et al. Safety measures during cholecystectomy: results of a nationwide survey. World J Surg 2011;35:1235-41; discussion 42-3.

22. Nijssen MA, Schreinemakers JM, Meyer Z, et al. Complications after laparoscopic cholecystectomy: a video evaluation study of whether the critical view of safety was reached. World J Surg 2015;39:1798-803.

23. Törnqvist B, Strömberg C, Akre O, et al. Selective intraoperative cholangiography and risk of bile duct injury during cholecystectomy. Br J Surg 2015;102:952-8.

24. Ford JA, Soop M, Du J, et al. Systematic review of intraoperative cholangiography in cholecystectomy. Br J Surg 2012;99:160-7.

25. Schols RM, Connell NJ, Stassen LP. Near-infrared fluorescence imaging for real-time intraoperative anatomical guidance in minimally invasive surgery: a systematic review of the literature. World J Surg 2015;39:1069-79.

26. Verbeek FP, van der Vorst JR, Schaafsma BE, et al. Image-guided hepatopancreatobiliary surgery using near-infrared fluorescent light. J Hepatobiliary Pancreat Sci 2012;19:626-37.

27. Agarwal BB. Patient safety in laparoscopic cholecystectomy. Arch Surg 2009;144:979.

28. Pesce A, Piccolo G, La Greca G, et al. Utility of fluorescent cholangiography during laparoscopic cholecystectomy: a systematic review. World J Gastroenterol 2015;21:7877-83.

29. Figueiredo JL, Siegel C, Nahrendorf $M$, et al. Intraoperative near-infrared fluorescent cholangiography (NIRFC) in mouse models of bile duct injury. World J Surg 2010;34:336-43.

30. Tagaya N, Shimoda M, Kato M, et al. Intraoperative exploration of biliary anatomy using fluorescence imaging of indocyanine Green in experimental and clinical cholecystectomies. J Hepatobiliary Pancreat Sci 2010;17:595-600.

31. Matsui A, Tanaka E, Choi HS, et al. Real-time intra-operative near-infrared fluorescence identification of the extrahepatic bile ducts using clinically available contrast agents. Surgery 2010;148:87-95.

32. Ishizawa $\mathrm{T}$, Bandai $\mathrm{Y}$, ljichi $\mathrm{M}$, et al. Fluorescent cholangiography illuminating the biliary tree during laparoscopic cholecystectomy. Br J Surg 2010;97:1369-77.

33. Aoki T, Murakami M, Yasuda D, et al. Intraoperative fluorescent imaging using indocyanine green for liver mapping and cholangiography. J Hepatobiliary Pancreat Sci 2010;17:590-4.

34. Verbeek FP, Schaafsma BE, Tummers QR, et al. Optimization of near-infrared fluorescence cholangiography for open and laparoscopic surgery. Surg Endosc 2014;28:1076-82.

35. Schols RM, Bouvy ND, van Dam RM, et al. Combined vascular and biliary fluorescence imaging in laparoscopic cholecystectomy. Surg Endosc 2013;27:4511-17.

36. Ashitate $Y$, Stockdale A, Choi HS, et al. Real-time simultaneous near-infrared fluorescence imaging of bile duct and arterial anatomy. J Surg Res 2012;176:7-13.

37. Mitsuhashi N, Kimura F, Shimizu H, et al. Usefulness of intraoperative fluorescence imaging to evaluate local anatomy in hepatobiliary surgery. J Hepatobiliary Pancreat Surg 2008;15:508-14.

38. Schols RM, Bouvy ND, Masclee AA, et al. Fluorescence cholangiography during laparoscopic cholecystectomy: a feasibility study on early biliary tract delineation. Surg Endosc 2013;27:1530-6.

39. Clavien PA, Barkun J, de Oliveira ML, et al. The Clavien-Dindo classification of surgical complications: five-year experience. Ann Surg 2009;250:187-96.

40. Benya R, Quintana J, Brundage B. Adverse reactions to indocyanine green: a case report and a review of the literature. Cathet Cardiovasc Diagn 1989;17:231-3.

41. Bjerregaard J, Pandia MP, Jaffe RA. Occurrence of severe hypotension after indocyanine green injection during the intraoperative period. A A Case Rep 2013;1:26-30.

42. Wolf $\mathrm{S}$, Arend $\mathrm{O}$, Schulte $\mathrm{K}$, et al. Severe anaphylactic reaction after indocyanine green fluorescence angiography. Am J Ophthalmol 1992;114:638-9.

43. Hope-Ross M, Yannuzzi LA, Gragoudas ES, et al. Adverse reactions due to indocyanine green. Ophthalmology 1994;101:529-33.

44. World Medical A. World Medical Association Declaration of Helsinki: ethical principles for medical research involving human subjects. JAMA 2013;310:2191-4. 
CHAPTER 3

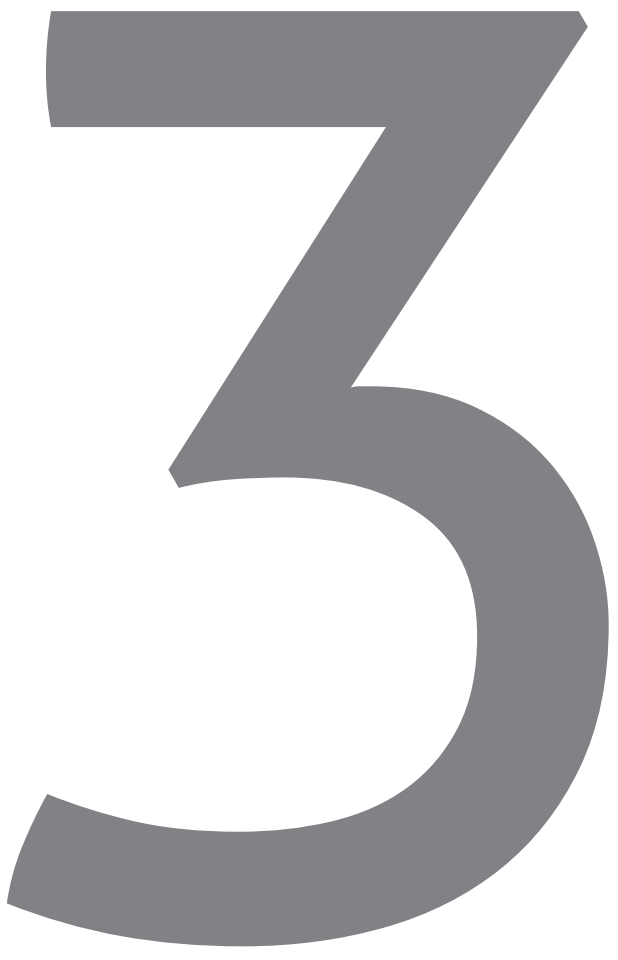




\section{Optimizing the image of Fluorescence Cholangiography using ICG: a systematic review and ex vivo experiments}

Jacqueline van den Bos, Fokko P. Wieringa, Nicole D. Bouvy, Laurents P.S. Stassen.

Surgical Endoscopy 2018; 32(12):4820-32 


\section{ABSTRACT}

\section{Background}

Though often only brie y described in the literature, there are clearly factors that have an influence on the fluorescence intensity, and thereby the usefulness of the technique. This article aims to provide an overview of the factors influencing the fluorescence intensity of fluorescence imaging with Indocyanine green, primarily focused on NIRF guided cholangiography.

\section{Methods}

A systematic search was conducted to gain an overview of currently used methods in NIRF imaging in laparoscopic cholecystectomies. Relevant literature was searched to gain advice on what methods to use. Ex vivo experiments were performed to assess various factors that influence fluorescence intensity and whether the found clinical advices can be confirmed.

\section{Results}

ICG is currently the most widely applied fluorescent dye. Optimal ICG concentration lies between 0.00195 and $0.025 \mathrm{mg} / \mathrm{ml}$, and this dose should be given as early as achievable—but maximum $24 \mathrm{~h}$-before surgery. When holding the laparoscope closer and perpendicular to the dye, the signal is most intense. In patients with a higher BMI and/or cholecystitis, fluorescence intensity is lower, but NIRF seems to be more helpful. There are differences between various marketed fluorescence systems. Also, no uniform method to assess fluorescence intensity is available yet.

\section{Conclusions}

This study identified and discussed several factors that influence the signal of fluorescence cholangiography. These factors should be taken into account when using NIRF cholangiography. Also, surgeons should be aware of new dyes and clinical systems, in order to benefit most from the potential of NIRF imaging. 


\section{INTRODUCTION}

The most common laparoscopic procedure in the Nether- lands is laparoscopic cholecystectomy (LC), which is per- formed 23,000 times each year. ${ }^{1}$ The most feared complication in this surgery is bile duct injury. Even though LC is a very common procedure, bile duct injury still has an incidence of $0.3-0.7 \% .^{2-5}$ Generally, bile duct injury leads to bile leakage, causing abdominal sepsis. It can also lead to obstruction, with obstructive jaundice, eventually potentially leading to a need for liver transplantation in the worst case. ${ }^{6}$ Late recognition is common in bile duct injuries, resulting in significant morbidity and mortality, a lower quality of life and extra costs. ${ }^{6-9}$ The main cause for bile duct injury is misidentification of the anatomy. ${ }^{10,11}$ Therefore, techniques to improve the visualization of the anatomy are desired.

Several imaging techniques to improve recognition of the relevant anatomical structures have been proposed. One is intra-operative cholangiography. Although this imaging technique is advised to reduce the risk of bile duct injury, there are disadvantages such as radiation exposure and need for additional equipment, knowledge and personnel to perform this procedure and extra costs. ${ }^{2,11-13}$ Because of these disadvantages, there is no worldwide consensus about implementation of intra-operative cholangiography. ${ }^{14}$

Another technique to help identify the relevant anatomical structures is the use of nearinfrared fluorescence (NIRF) imaging. This is a relatively new and promising technique to improve recognition of the biliary anatomy by injecting a fluorescent dye. ${ }^{15,16}$ The clinically used dye is Indocyanine green (ICG), belonging to the family of cyanine dyes. ${ }^{17}$ ICG is a watersoluble tricarbocyanine with a molecular weight of 774.96 absorbing excitation light at 800-835 nm while emitting fluorescent light at $740-793 \mathrm{~nm} .{ }^{18-20}$ Generally, ICG is considered safe with a very low incidence of complications, although there is a possibility of an allergic reaction to the sodium-iodine in the ICG. ${ }^{21-23}$ Other dyes are not generally available yet, either because they are not excreted in bile or because they are still in a research phase and thus not yet available for routine clinical use. ${ }^{24,25}$

In addition to this small risk for an allergic reaction, all researchers and surgeons working with near-infrared (NIR) fluorescence-imaging experience limitations of the technique. Though often only briefly described in the literature, there are clearly factors such as the given dose and distance between the laparoscope and the target that have an influence on the fluorescence intensity, and thereby the usefulness of the technique. Since this technique is nowadays commonly used, it is of importance to obtain more general knowledge about the factors that influence the fluorescence intensity. Therefore, this article aims to provide an overview of these factors influencing the fluorescence intensity, primarily focused on NIRF guided cholangiography during laparoscopic cholecystectomy. 


\section{MATERIALS AND METHODS}

To provide an overview of the factors influencing the fluorescence intensity in NIRF-guided cholangiography during laparoscopic cholecystectomy, three strategies were combined. First a systematic review of the literature was performed. Secondly, using free searches, more information about specific suspected factors of influence was sought. Thirdly, factors of influence identified from the literature were further investigated with ex vivo experiments. Since no (new) patients are included in this study, IRB approval or patients informed consent was not needed for this study.

\section{Literature search}

A Pubmed search on methods used for fluorescence cholangiography and its influencing factors was performed by two reviewers through November 2017. Used search terms were as follows: ((()(()((((near-infrared imaging) OR fluorescence imaging) OR fluorescent imaging) OR near-infrared fluorescence) OR fluorescent dyes) OR fluorescent dye) OR "Fluorescent Dyes"[Mesh]) OR ICG) OR infracyanine green) OR indocyanine green) OR "Indocyanine Green"[Mesh])) AND ((((((“Cholecystectomy“[Mesh]) OR Cholecystectomy) OR laparoscopic cholecystectomy) OR "Cholecystectomy, Laparoscopic"[Mesh]) OR gallbladder removal) OR laparoscopic cholecystectomies). No Language restrictions were applied. Studies presenting original data on performing near-infrared fluorescence-guided cholecystectomy in human subjects were included. Therefore, animal studies, protocol-articles, reviews and case reports were excluded. From the included reports, data regarding the used methods and outcomes (visibility of anatomical structures) was extracted.

\section{Ex vivo experiments}

In addition, ex vivo experiments were performed to further analyze certain aspects. The aim of these experiments was to evaluate whether albumin is needed in the medium used for ICG to become fluorescent and to study the influence of the concentration of ICG, the distance between the laparoscope and the target, the angle between the laparoscope and the target and the penetration depth on the fluorescent signal.

A laparoscopic Near-infrared Fluorescence Imaging System (Karl Storz GmbH, Germany) was used, as we use in daily clinical practice. This system includes a D-light $\mathrm{P}$ xenon light source, an IMAGE 1 camera and a 30-degree $10 \mathrm{~mm}$ fluorescence laparoscope (Karl Storz $\mathrm{GmbH}$, Germany).

Experiments were performed both with ICG diluted in $\mathrm{NaCl} 0.9 \%$ and with ICG dilution in $35 \mathrm{mg} / \mathrm{ml}$ albumin in a $0.9 \% \mathrm{NaCl}$ dilution. This was done as ICG is considered in vivo to bind to albumin. The aim was to assess whether such binding ex vivo was necessary in order to obtain a signal.

Initially, we used ten different concentrations of ICG, ranging between 10 and $0.125 \mathrm{mg} / \mathrm{ml}$. 


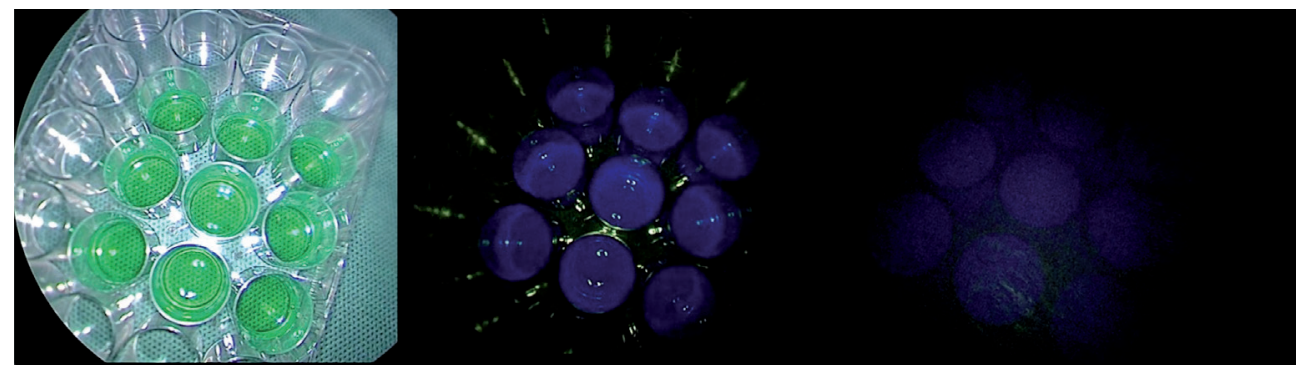

9 times $1 \mathrm{ml}$ of ICG in a dilution of $0.125 \mathrm{mg} / \mathrm{ml}$ in the cups of a Wells plate. Left: in white light. Middle: in NIRF light. Right: in NIRF light with one plate $(0.9 \mathrm{~mm})$ of beeswax on top of the Wells plate.

Figure 1 Setup of ex vivo experiments

In these experiments, a lower concentration gave a better signal. To find the optimum, eventually twenty-one solutions ranging from 10 to $0.000121875 \mathrm{mg} / \mathrm{ml}$ were used. From each dilution, 9 times $1 \mathrm{ml}$ of the ICG was placed on a wells plate.

The influence of distance was measured holding the endoscope at distances from 14 to $1 \mathrm{~cm}$ from the surface of the dye. Also, the maximum distance at which the fluorescence could still be detected was measured for each concentration.

The influence of angle between the laparoscope and the surface of the dye was measured by comparing the middle cup, at which the laparoscope was aimed, with the surrounding cups with ICG and by holding the endoscope at different angles.

The penetration depth was evaluated with the use of beeswax plates stacked to different thicknesses. This medium was chosen because it approaches the scattering behavior and translucent light penetration of human tissue quite well. ${ }^{26}$ In fact, from the renaissance until the early twentieth century, anatomical wax models were used for medical education and until today provide an impressive visual display. ${ }^{27}$ Beeswax plates of exactly $0.9 \mathrm{~mm}$ thickness were one by one progressively stacked on the wells plate to stepwise increase the thickness of scattering material on top of the fluorescent dye. In all experiments, fluorescence intensity was measured using Osirix (version Lite 8.5.2, Pixmeo). The setup of the ex vivo experiment is shown in Figure 1.

Additional information for this article was retrieved by a free search in the PubMed database, hand-searches of the retrieved references and personal experience with the technique.

\section{RESULTS}

The systematic search was performed to retrieve insight in the currently used methods of performing near-infrared fluorescence guided cholangiography and the factors influencing its signal intensity. This search resulted initially in 126 articles, of which 80 were excluded 
based on title and abstract and 18 more after full text assessment. 28 articles were included in this review. Figure 2 presents the flowchart of this search. ${ }^{13,19,28-53}$ An overview of the included articles and their results is given in Tables 1 and 2 (Supplementary Material), respectively.

The results of literature search and the results of the performed experiments will be discussed in this section.

\section{Fluorescent dye and concentration}

In all studies, ICG is used as the fluorescent dye. Both Indocyanine green and Infracyanine green where used. ICG was administered intravenously, except in the study by Graves et al. ${ }^{29}$ and Liu et al. ${ }^{53}$, who injected the fluorescent dye directly into the gallbladder. Graves et al. made a special solution for this intra-gallbladder injection. First, a $0.25 \mathrm{mg} / \mathrm{ml}$ solution of ICG in sterile water was created. Once the gallbladder fundus was grasped and retracted, a needle tipped Kumar cholangiogram catheter (Nashville Surgical Instruments, Springfield, TN) was introduced through the $12-\mathrm{mm}$ port and used to puncture the infundibulum of the gallbladder. $9 \mathrm{ml}$ of bile was aspirated from the gallbladder through this catheter and mixed with the $0.25 \mathrm{mg} / \mathrm{ml}$ ICG solution. The new $0.025 \mathrm{mg} / \mathrm{ml} \mathrm{ICG-bile} \mathrm{solution} \mathrm{was} \mathrm{reinjected}$ into the gallbladder. The authors describe an immediately glow-up of the solution with no lag time, quickly filling the gallbladder and through the cystic duct the extrahepatic ducts. Due to the absence of background fluorescence from the liver, improved visual contrast was claimed as compared to intravenous injection. ${ }^{29}$ Liu et al. used a less complicated method, in which the ICG was diluted to a concentration of $0.125 \mathrm{mg} / \mathrm{mL} .10 \mathrm{~mL}$ of this ICG dilution was injected through a gallbladder drain when this was already in situ, or through a Veress needle after draining the gallbladder from bile..$^{53}$ In these patients, although background fluorescence was absent, results comparable to intravenous administration were found.

The most commonly used doses of ICG for intravenous administration where a fixed dose of $2.5 \mathrm{mg}$ bolus (in 13 of the studies) or a dose adjusted to the patient's weight of 0.05 $\mathrm{mg} / \mathrm{kg}$ (in 6 of the included studies). Other used doses are fixed dosed of $0.25,3.75,5,10$ and $12.5 \mathrm{mg}$ and weight-adjusted doses of $0.1-0.4,0.2,0.4$ and $0.5 \mathrm{mg} / \mathrm{kg}$ (see Table $1 \mathrm{in}$ Supplementary Material). When using a $2.5 \mathrm{mg}$ bolus, the cystic duct was visualized in an average of $94 \%$ patients. When using $0.05 \mathrm{mg} / \mathrm{kg}$, the average percentage of visualization of the cystic duct was $98 \%$.

Zarrinpar et al. ${ }^{54}$ undertook a study with systematic variation of dosing (and timing) of injection of ICG. In 37 patients, the given dose varied between 0.02 and $0.25 \mathrm{mg} / \mathrm{kg}$. With an increasing dose, the visualization of the extrahepatic biliary tract improved. No decrease at the highest doses was observed. Boogerd et al. ${ }^{30}$ compared a dose of 5 and $10 \mathrm{mg}$ with optimal signal at the lower dose of $5 \mathrm{mg}$.

The results of our ex vivo experiments are depicted in Figure 3. When ICG was diluted in $\mathrm{NaCl} 0.9 \%$, no fluorescent signal could be obtained. Therefore, all further experiments 


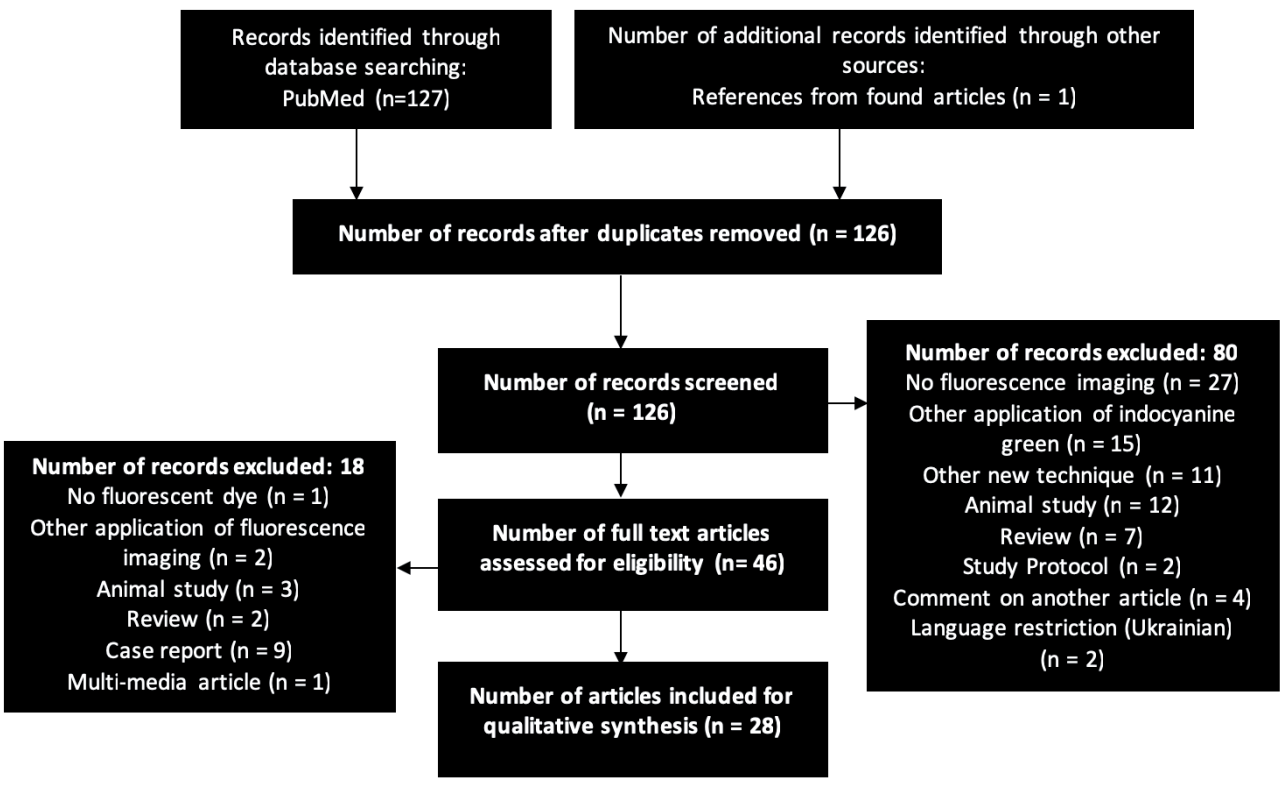

Figure 2 Flow chart of search
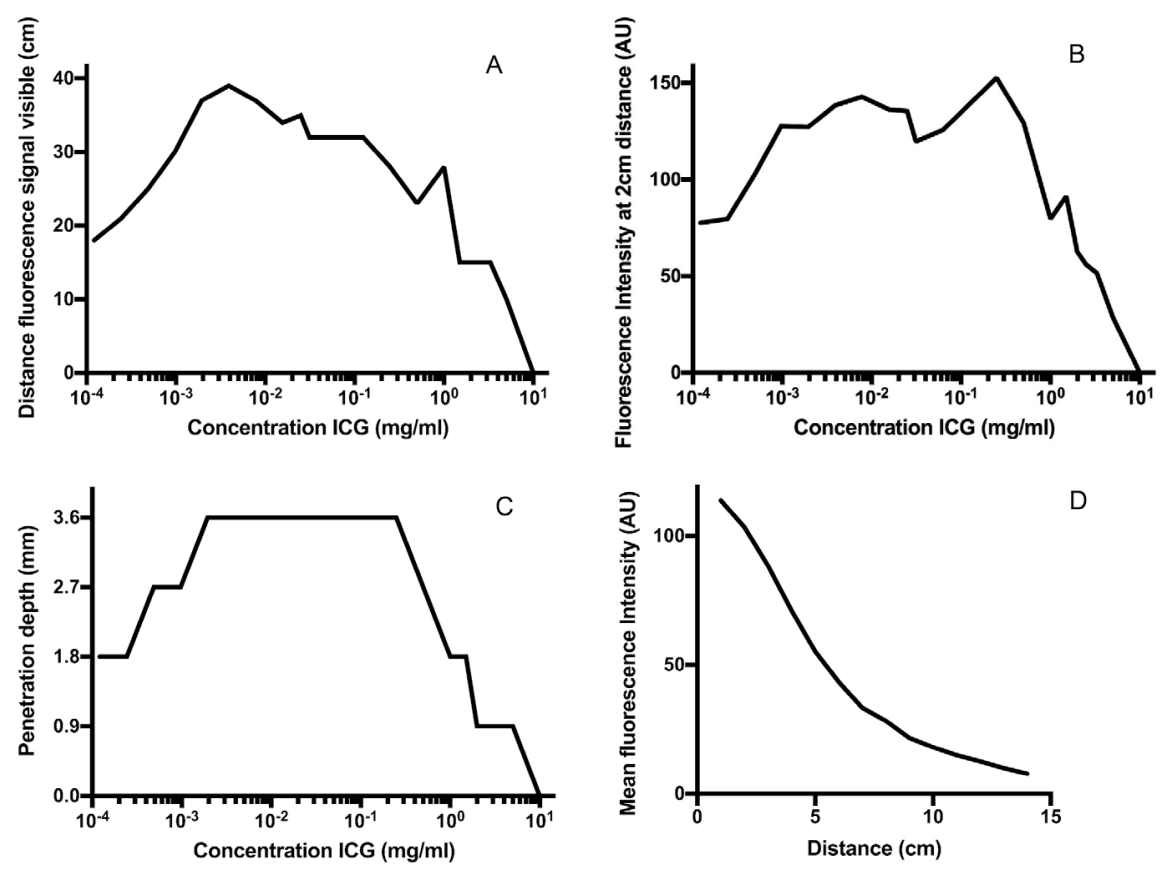

Legend: A Maximum distance of visibility fluorescent signal for the tested concentrations

B Fluorescence intensity at $2 \mathrm{~cm}$ distance for the tested concentrations

C Maximum penetration depth of fluorescent signal for the tested concentrations

D Fluorescence intensity at increased distance between laparoscope and dye-surface

Figure 3 Results of ex vivo experiments 
were performed with ICG diluted in $35 \mathrm{mg} / \mathrm{ml}$ albumin in a $0.9 \% \mathrm{NaCl}$ solution. The optimum concentration in which the highest measured fluorescence intensity at $2 \mathrm{~cm}$ distance was between 0.00195 and $0.025 \mathrm{mg} / \mathrm{ml}$. A concentration of $0.0039 \mathrm{mg} / \mathrm{ml}$ would be a dose of $19.5 \mathrm{mg}$ to obtain this ideal concentration in the blood in an average patient with $5 \mathrm{~L}$ blood, which is much higher than current used concentrations. However, when using this technique in laparoscopic cholecystectomy, we would want the concentration of $0.0039 \mathrm{mg} / \mathrm{ml}$ in the cystic duct. With a content of $60 \mathrm{ml}$ of bile in the gallbladder, a dose of $0.234 \mathrm{mg}$ would then be desirable assuming all ICG is cleared by the liver.

The used concentration of the administered ICG solution was not clearly described in every study. The studies that do provide information on ICG concentration use $2.5 \mathrm{mg} / \mathrm{ml} .^{19}$, $33,34,40,46,49-52$ In all, except two studies, the fluorescent dye was administered intravenously.

\section{Timing of administration}

The timing of administration differed among the studies and ranged mostly between 1 and $2 \mathrm{~h}$ and $15 \mathrm{~min}$ before surgery. Verbeek et al. ${ }^{55}$ investigated whether a longer period between the administration of ICG and the imaging resulted in an improved visibility of the biliary anatomy. They found that application $24 \mathrm{~h}$ prior to surgery does result in a significant better signal-to-background ratio. ${ }^{55}$ This is mainly caused by a lesser fluorescent signal coming from the liver. Kono et al. ${ }^{56}$ found a significant difference in interval between injection and imaging between patients in whom the cystic duct-common hepatic duct confluence could be visualized by NIRF imaging and the patients in whom this could not, with median intervals of 90 (15-65) minutes and 47 (21-205) minutes, respectively. The above is in line with the advice of Boogerd et al. ${ }^{30}$, for an optimal time interval of $3 \mathrm{~h}$ between administration of ICG and intra-operative fluorescence cholangiography.

\section{Penetration depth}

Circumstances that result in thicker tissue covering the structures to identify are obesity and cholecystitis. These require a higher penetration depth of the signal.

Average $\mathrm{BMI}$ in the included studies ranged between 20.4 and $32.3 \mathrm{~kg} / \mathrm{m}^{2}$ with a weighted average of $30.0 \mathrm{~kg} / \mathrm{m}^{2}$ for all studies who report mean BMI. Dip et al. ${ }^{35}$ specifically investigated the accuracy of NIRF-guided surgery in morbidly obese subjects undergoing laparoscopic cholecystectomy. No differences in hepatic duct, common bile duct and accessory duct visualization were detected in the obese and non-obese groups ( $p$ value $0.09,0.16$ and 0.66 , respectively). Imagi et al. ${ }^{34}$ state that obesity is an important factor that can prevent identification of biliary structures under fluorescent cholangiography. Other studies notice that when not all aimed structures where visualized under

fluorescent cholangiography in every patient, the patients in which the structures were not visible were all obese. ${ }^{40,42,44,51}$ Ankersmit et al. ${ }^{31}$, however, found no differences in ability to visualize the biliary structures in obese patients. Their hypothesis is that other patient 
and surgical-related factors, including inflamed tissue, may interfere with and influence the success rate of fluorescence visualization of the bile ducts in complicated cases. ${ }^{31}$

Of the included patients in the studies found, $17 \%$ had acute cholecystitis. Four of the studies make a comment about the influence of cholecystitis on the usefulness of the NIRF technique. Zroback et al. ${ }^{33}$ note that in their experience, during dissection of the most inflamed gallbladders was when the technology was most appreciated, but no specific remark is made on the hindrance by thickened tissue. The other articles mention that the patients in which the biliary structures were not visible with NIRF more often had cholecystitis than the patients in which they were visible. ${ }^{13,39,42}$ Although in several studies the indication for surgery was also cholecystitis and the number of patients with this condition was given, no specific comparison was made with regard to the successful imaging of the desired structures between the patients with and without cholecystitis in most studies. Liu et al. ${ }^{53}$ make this comparison and conclude that in these patients, NIRF helped to significantly improve the visualization rates of the cystic duct in patients with cholecystitis compared to white light, whilst in cases with lithiasis without inflammation, the visualization rate of the cystic duct was similar in NIRF and white light.

In our ex vivo experiments, the penetration depth was studied at all concentrations separately. We observed a correlation between the thickness of material to be penetrated and the resulting signal, with an optimum and a maximum. A maximum penetration depth of $3.6 \mathrm{~mm}$ was measured. The optimum is at the same concentrations as we observed in our experiments on the concentration of ICG namely between 0.00195 and $0.025 \mathrm{mg} / \mathrm{ml}$. See also Figure 3.

\section{Distance to the target}

Kono et al. ${ }^{56}$ described a decrease by $50 \%$ of the contrast signal when the distance from the bile duct and tip of the laparoscope increased from 5 to $15 \mathrm{~cm}$. However, other manuscripts do not clearly describe the distance used between the bile ducts and the laparoscope.

In our ex vivo experiments, a lower fluorescence intensity was found in all concentrations when the laparoscope was held at a greater distance. A 5 up to 30 times lower fluorescence intensity was observed when increasing the distance from 5 to $14 \mathrm{~cm}$ of the ICG solution, as is illustrated in Figure 3. When looking at the different concentrations, the maximum distance at which the fluorescent signal was still detectable was higher at the 'optimal concentrations' as shown in Figure 3.

\section{Angle between laparoscope and tissue}

Kono et al. ${ }^{56}$ point out that the tip of the laparoscope should be placed vertically to Calot's triangle to directly irradiate exciting light on the bile ducts and obtain the optimal fluorescence signal. However, as with the distance of the laparoscope, other manuscripts do not mention whether the laparoscope was placed vertically or in another angle. Some of the 
articles use a zero-degree laparoscope, while others use a 30-degree laparoscope, which may be of influence on the angle of examination. In our experiments, a higher fluorescence intensity was found when holding the laparoscope more vertically towards the tissue.

\section{Differences between systems}

The systems used in the included studies were 10 times a system by Karl Storz; 6 times the Firefly system; 3 times an Olympus system; 5 times a Hamamatsu system and 2 Stryker systems. Two studies did not mention which system was used. When comparing the studies using the different systems studies using Karl Storz can visualize the cystic duct in 93\%; Firefly in 97\% Olympus in 92\% Hamanatsu in 92\% and Stryker in 94\%.

Kono et al. ${ }^{56}$ compared five imaging systems. Namely, a prototype fluorescence imaging system from Hamamatsu Photonics (Hamamatsu, Japan and Shinko Optical, Tokyo, Japan), the system from Olympus Medical Systems (Tokyo, Japan), thirdly a high-definition model From Hamamatsu Photonics (Hamamatsu, Japan and Shinko Optical, Tokyo, Japan), the highdefinition fluorescence imaging system from Karl Storz (Tuttlingen, Germany) and fifth, the high-definition imaging system from Novadaq (Toronto, Canada). A significant difference between these systems tested for signal contrast was found. ${ }^{56} \mathrm{It}$ is not clear whether the systems tested are exact alike with the systems that are at present commercially available and clinically used.

\section{Interpretation of the signal}

Most studies only state whether a certain anatomical structure is visible or not, without quantification of the fluorescence intensity. There is no generally applied analytic measure to objectify the fluorescence intensity yet. When the fluorescence intensity is measured in literature, several methods based on the color-intensity are used. In most of these methods, only the fluorescence intensity of the target is measured.

Schols et al..$^{43,46}$ uses in both articles measurement of the Target to Background Ratio (TBR) to objectify the degree of fluorescence illumination. For this, OsiriX 5.5.1 Imaging Software was used. The TBR was defined as the mean fluorescence intensity (FI) of twopoint regions of interest (ROIs) in the target (i.e. $C B D, C D$, or $C A$ ) minus the mean FI of two background ROls in the liver hilum, divided by the mean FI of the two Background ROls in the liver hilum; The used formula was as follows: TBR = (FI of target-FI of background)/FI of background.

Ashitate et al. ${ }^{57}$ apply the same formula, but do not mention which software was used. Also, the exposed rectus muscle was used as background ${ }^{57}$. Objectively this is correct, as any consistent background can be chosen, but subjectively, it is more logical to use the liver as background, as this is the actual observed background during the operation.

Kono et al. ${ }^{56}$ also use a signal to background ratio. In their series, Photoshop CS5 software (Adobe Systems, San Jose, CA) was used to calculate the fluorescence intensity in 
the regions of interest with a range of $0-255$ from still images. The used formula here was as follows: Signal contrast $=(\mathrm{Fl}$ in fluorescence regions-FI in background $) / 225$.

Zarrinpar et al. ${ }^{54}$ used ImageJ (US National Institutes of Health, Bethesda, MD; http:// image j.nih.gov/ij/) software to calculate intensity ratios. They divided the fluorescence intensity signal of the common bile duct by that of the surrounding fat or liver. Verbeek et al. ${ }^{55}$ use the same formula as Zarrinpar, but do not mention which software was used.

\section{DISCUSSION}

The aim of this article is to give an overview of factors influencing the signal intensity during fluorescence imaging, primarily focused on NIRF-guided cholangiography with ICG during laparoscopic cholecystectomy. These influences can be divided in patient factors, the applied fluorescent dye, the equipment and the method of assessing fluorescence intensity. Regarding patient factors, obesity and inflammation, i.e. cholecystitis, are of importance. These may cause thickening of tissue covering the structures to be visualized, thereby decreasing signal intensity. Although fluorescence imaging has a better tissue penetration than white light, due to its emission in the near-infrared wavelength zone, penetration is limited by such factors.

In several studies, patients with cholecystitis were included. In four studies, a comment is given about the influence of cholecystitis on the usefulness of the NIRF technique.

Zroback et al. ${ }^{33}$ appreciated the NIRF technology most while dissecting the most inflamed gallbladders. On the contrary, three other authors mention that in patients in whom the biliary structures could not be visualized, more often cholecystitis was present than in patients in whom imaging was successful ${ }^{13,39,42}$. Liu et al. report a significant difference in visibility of the cystic duct with NIRF compared to white light in patients with cholecystitis. ${ }^{53}$ This is an indication that NIRF imaging might be more helpful in cholecystitis patients. No difference in complications due to NIRF imaging was reported between patients with and without cholecystitis.

Obesity may result in more fatty tissue overlying the extrahepatic biliary tree. The average $\mathrm{BMI}$ in the included studies ranged between 20.4 and $32.3 \mathrm{~kg} / \mathrm{m}^{2}$ with an overall average of $30.0 \mathrm{~kg} / \mathrm{m}^{2}$. Considering this high average $\mathrm{BMI}$ and the good results the manuscripts describe; a high BMI seems not to be a burden in the use of NIRF cholangiography. Dip et al. ${ }^{35}$ specifically investigated the accuracy of NIRF guided surgery in morbidly obese subjects undergoing laparoscopic cholecystectomy and could not find differences in biliary duct visualization between the obese and the non-obese groups. ${ }^{35}$ Some studies confirm this absence of influence of BMI on the visualization scores. ${ }^{54,56}$ On the contrary, other studies claim that BMI has a negative influence on the visibility of the structures using NIRF. $34,40,42,44$, ${ }^{51} \mathrm{It}$ is even suggested that also a more common inflammatory response is present in these 
patients. ${ }^{42}$ The practice of using fluorescence cholangiography is that it is applied while gradually dissecting the surgical area. Sooner or later during this dissection, NIRF will lead to confirmation of the anatomical structures. More fatty tissue requires more dissection andmost likely-later visualization of structures. The earlier or later recognition may influence the surgeon's satisfaction with the NIRF technique. Another explanation for differences between studies may be that the signal of ICG depends on the concentration used. ${ }^{58} \mathrm{It}$ is possible that in patients with a higher bodyweight, the concentration of ICG differs when using a fixed and not a weight-adjusted dose. This will be discussed hereunder.

In the performed ex vivo experiments, a maximum penetration depth of $3.6 \mathrm{~mm}$ was achieved. This confirms the limits of penetration of the NIRF signal. However, bees wax was used to measure the penetration depth. Although spectral properties are similar to human tissue, it is possible that the penetration depth in human tissue is different. Anyhow, this in vitro method at least offers an objective and simple tool to compare the performance of different laparoscopes, light sources, and camera systems.

In conclusion, NIRF seems also to be beneficial in circumstances where penetration depth is limited due to thickened overlying tissue. In these cases, the image will be obtained later, after relatively more dissection has been performed. The surgeon should be aware of this, but with this in mind, can use the technique to enhance recognition of the essential structures.

Concerning the fluorescent dye used, several factors may be of influence on the signal obtained: the type of dye, the dose used and its concentration, the timing and the route of administration.

Regarding the type of dye, there are several factors that determine the effectiveness of a fluorescent dye. These are probe targeting (a dye for bile duct imaging should be able to reach the bile ducts and to some extent accumulate there), activation, pharma kinetics (how fast is the dye at the desired place and how long will it stay there?), biocompatibility and photo physics. ${ }^{59}$ The dye should ideally be in the near-infrared range, since this offers the spectral range with best light penetration (so-called near-infrared window). Also, the use of a near-infrared filter and light source eliminate autofluorescence. ${ }^{60}$ Wavelengths below $600 \mathrm{~nm}$ encounter this higher auto-fluorescence due to the presence of many endogenous fluorophores and strong scattering. Below $600 \mathrm{~nm}$, there is a strong absorbance of haemoproteins such as haemoglobin, myoglobin and cytochromes causing less deep tissue penetration. ${ }^{59,61}$

Indocyanine green is currently the only clinically used dye for fluorescence cholangiography, illustrated by the fact that it was used in all 27 studies of this review. In most of these studies, ICG was mostly an abbreviation for indocyanine green, but in four studies the iodine-free infracyanine green was used. The absence of iodine in infracyanine seems to make it less toxic and available for patients with an iodine allergy. We did not perform in vitro experiments with infracyanine green, but the absorption and emission spectra of both dyes do not differ ${ }^{62}$, and thus the signal is expected to be equivalent. 
Another previously tested fluorescent dye is Methylene Blue (MB). This has not been used in the studies reported. MB and ICG have been compared in an animal study using pigs in which both dyes were directly injected into the gallbladder through a 10 Fr catheter that was inserted into the gallbladder fundus. ${ }^{63}$ Using this method, ICG had a higher target to background signal, due to a higher extinction coefficient and higher fluorescence rate. An advantage of $M B$ is limited liver uptake resulting in less background signal. The signal becomes visible within minutes and remains adequate for $120 \mathrm{~min} .{ }^{63} \mathrm{~A}$ disadvantage of $\mathrm{MB}$ is the absorption and emission around $700 \mathrm{~nm}$ which is subject to a higher background auto-fluorescence ${ }^{63}$ but also requires different settings of the equipment. Another drawback is the need for intra-gallbladder administration of the dye, since methylene blue is cleared by the kidneys, not the liver. ${ }^{64}$ Ashitate et al. ${ }^{57}$ combined two dyes in an animal study. Herein, they compared three different combinations; first methylene blue for visualization of the cystic artery and indocyanine green for visualization of the bile duct; secondly ICG for the artery and Methylene blue for the bile duct; thirdly ZW-800-1 for the artery and methylene blue for the bile duct. The authors' conclusion is that the third combination had the best overall performance, and the second combination was the best combination of clinically available dyes. ${ }^{57}$

Apart from the above mentioned ZW-800, other new preclinical dyes such as VM678, IRDye ${ }^{\oplus 800 C W}$, IRDye ${ }^{\oplus} 800$ BK and IRDye ${ }^{\oplus} 800$ NOS have been tested in animals and show very promising results. ${ }^{24,65}$ These are due to better pharmacokinetic characteristics and targetto-background ratio of these dyes. ${ }^{24,66}$ The IRDyes have the same spectral characteristics as ICG, which enables the use of the same equipment. IRDye ${ }^{\circledR} 800 \mathrm{CW}$ is expensive, with a price tenfold that of ICG; IRDye ${ }^{\circledR} 800 \mathrm{BK}$ and IRDye ${ }^{\circledR} 800$ NOS however cost the same as ICG. ${ }^{65}$ But these dyes are not yet FDA approved.

In conclusion, ICG is currently the most used and preferred dye. Only limited data are available on MB and it is not used in clinical practice for cholangiography, probably based on the characteristics described above. New preclinical dyes are promising but await approval for clinical practice.

The dose used may also be of influence on the performance of the dye. In the studies presented, a bolus was given (2.5 mg in 13 studies, $12.5 \mathrm{mg}$ and $3.75 \mathrm{mg}$ in two others); or a weight-adjusted dose $(0.05 \mathrm{mg} / \mathrm{kg}$ in seven studies and $0.04 \mathrm{mg} / \mathrm{kg}$ in three other studies).

Zarrinpar et al..$^{54}$ undertook a prospective study with systematic variation of dosing and timing from injection of ICG to visualization. In 37 patients, doses were given varying between 0.02 and $0.25 \mathrm{mg} / \mathrm{kg}$. Their conclusion was that with increasing dose, the visualization of the extrahepatic biliary tract improved. Visualization was also better with increased time after ICG administration (10 min vs. 45 min vs. 3h). Boogerd et al. ${ }^{30}$ compared a dose of 5 and 10 $\mathrm{mg}$ and advise to use the lower dose of $5 \mathrm{mg}$.

Our ex vivo experiments suggest that the $2.5 \mathrm{mg}$ bolus that is used in most studies, is probably below the optimal clinical dose. One should however take into account that these ex vivo experiments are a controlled setting, which is not entirely comparable to the 
complex in vivo biological system in which we use the NIRF cholangiography. Therefore, a recommendation regarding the optimal dose cannot be given based on these experiments. Nevertheless, the conclusion can be drawn that applying a weight-adjusted dose seems preferable over a fixed dose.

The used concentration of the administered ICG solution was not clearly described in

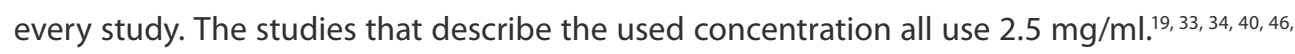
${ }^{49-52}$ This is remarkable since the leaflet that comes with ICG advises to dilute the ICG to a solution of $5 \mathrm{mg} / \mathrm{ml}$. However, a concentration of $2.5 \mathrm{mg} / \mathrm{ml}$ is more feasible in daily practice since $1 \mathrm{ml}$ can be retrieved more precise than just $0.5 \mathrm{ml}$.

Hiratoglou et al. ${ }^{62}$ investigated the influence of the concentration and solvent medium on the light-absorbing prosperities in ICG. When glucose $5 \%$ was used as a solvent medium, the absorption between 600 and $700 \mathrm{~nm}$ was decreased, compared with the absorption with Balanced Salt Solution (BSS) or BSS Plus. These differences between BSS/BSS Plus- and glucose 5\%-diluted ICG decreased when the concentration was lowered. They conclude that depending on the used solvent medium, the absorption spectrum of ICG changes with different concentrations. ${ }^{62}$ Based on these findings, it might be advisable to use sterile water as a solvent medium, as most groups currently do, and not a glucose solution.

Kono et al. ${ }^{56}$ found a lower fluorescence intensity when using a more diluted solution of ICG. Our ex vivo experiments show an optimum of ICG concentration. This optimum seems to be at a higher concentration than is currently achieved in the clinical situation.

However, this conclusion is an extrapolation of the experimental data to the clinical situation for the concentration of ICG in the blood. The extrapolation may not be correct and is also not directly translatable to the concentration in the bile ducts. And one must be aware that not so much the concentration of the solution applied, but the total dose administered determines the systemic concentration of the dye.

The timing of administration ranged mostly between 2 hours and 15 minutes before surgery. Verbeek et al..$^{55}$ investigated whether a longer time between the administration of ICG resulted in an improved visibility of the biliary anatomy. The authors found that administration $24 \mathrm{~h}$ prior to surgery results in a significantly better signal to background ratio. ${ }^{55}$ This is mainly caused by a lesser fluorescent signal coming from the liver. A problem with this approach is the practical applicability: in day care admissions and in acute cholecystitis an injection $24 \mathrm{~h}$ before surgery often is not feasible. Also, since only $30 \mathrm{~min}$ versus $24 \mathrm{~h}$ before surgery were tested, the optimum may lie between these timeslots. Kono et al. ${ }^{56}$ and Boogerd et al. ${ }^{30}$ also conclude that earlier administration results in better visualization. In conclusion, administration closer to the time of surgery can result in a more prominent background signal from the liver. Therefore, administration as early as possible (but not earlier than 24h) should be considered.

In all studies, except for the study by Graves et al. ${ }^{30}$ and Liu et al. ${ }^{53}$, the fluorescent dye was administered intravenously. This is easy and convenient for the patient, since 
intravenous access is needed anyway for induction of anesthesia. However, systemic injection results in the disadvantage of occurrence of (background) fluorescence of the liver, which considerably decreases the signal to background ratio. Another limitation of this administration route is the dependency on the refilling of the gallbladder and cystic duct with fluorescent bile by reflux, which is unpredictable. ${ }^{38} \mathrm{~A}$ solution might be direct injection of ICG into the gallbladder. Lui et al. ${ }^{67}$ previously examined the feasibility of this technique in pigs. They conclude that fluorescence cholangiography through direct intragallbladder injection can rapidly provide an adequate visualization of the gallbladder neck and cystic duct. The problems with a high background signal from the liver and the needed time for the ICG to be extracted from the blood and secreted into the biliary system are solved by this administration route. This is in line with their newer study in humans ${ }^{53}$ and the study by Graves et al. ${ }^{10}$, who injected the Indocyanine green in eight patients and achieved comparable encouraging results as described in the animal study. However, spill of ICG at the puncture site of the gallbladder may decrease overall visibility of the structures to be visualized. This is illustrated by the remark of Osayi ${ }^{40}$ that in three of the patients the visualization was impaired due to bile leakage from the gallbladder, albeit that this was not caused by puncture, but by surgical trauma.

With regard to the imaging system, the distance and the angle between the endoscope and the target, and the properties of the system, have influence on the signal.

The distance between the fluorescent dye and the laparoscope seems to be of influence on the fluorescence intensity. Kono et $\mathrm{al}^{56}$ describe a decrease by $50 \%$ of the signal when the distance between the laparoscope and the target increased from 5 to $15 \mathrm{~cm}$. Other manuscripts do not describe the distance between the bile ducts and the laparoscope. In our experiments, a clear relation was found between the distance of the laparoscope to the target and the measured fluorescence intensity (Figure 3). In practice, the distance at which the endoscope is held, is primarily dictated by the optimal overview for surgical dissection. Ideally, the properties of the dye and the system enable the surgeon to maintain the same distance for dissection and for NIRF imaging. However, the surgeon must be aware that for optimal fluorescence signal temporarily adjusting of the distance may be necessary.

Kono et $\mathrm{al}^{56}$ point out that the tip of the laparoscope should be held vertically to Calot's triangle to directly irradiate exciting light on the bile ducts to efficiently obtain fluorescence signals. However, as with the distance of the laparoscope, other manuscripts do not mention whether the laparoscope was held vertically or in another angle. Some of the articles use a zero-degree laparoscope, while others use a 30-degree laparoscope, which is also a factor that should be kept in mind when aiming for the optimal angle.

In the ex vivo experiments, a better signal was seen when the laparoscope's central viewing axis was aligned perpendicular to the target (i.e. an angle between the tip of the laparoscope and the fluid level in het Well's plate of 90 degrees). Because a 30-degree laparoscope was used, this needed to be held at 120 degrees to obtain the highest 
fluorescence intensity. This confirms the clinical findings of Kono et al. ${ }^{56} \mathrm{As}$ with the distance, the position of the endoscope is mainly aimed at facilitating dissection. At moments of attention to the NIRF signal, focus should also be on the 90-degree angle to the target.

Five different systems were used in the included studies. As discussed before, the tissue depth to which NIRF imaging can detect agents is dependent upon their brightness and the sensitivity of the device. ${ }^{68}$ The main components of a fluorescent imaging device are a spectrally adjusted light source for exciting ICG; optical filters for separation of the emitted fluorescent light and the background scattering and ambient light; camera(s) for detecting the emitted fluorescent signals; display software and display hardware. ${ }^{69}$ The performance of a device is mainly determined by these components. ${ }^{68}$

The most used light source type in the included studies is a xenon light for laparoscopy, and a laser diode in the robotic cases. Laser diodes enable the greatest sensitivity since the background due to background excitation light is the lowest. A xenon light source has a very broad bandwidth and has therefore a lower sensitivity. ${ }^{68}$ The detection sensitivity in all fluorescent imaging systems is limited by the background signals. There is a spectral overlap between the backscattered excitation light and emitted fluorescence signals. In this way, non-fluorescent signals can be seen as fluorescence. Another problem is a limited optical density that allows passage of a small amount of ambient light, also called filter light leakage. This causes a 'noise floor', which makes it less probable that a device can detect small amounts of the fluorescent dye in tissues. ${ }^{68,70} \mathrm{~A}$ sufficient level of noise floor is needed to identify tissues of the surrounding, but when the noise floor is close to the fluorescent signal, the sensitivity of the imaging device and depth penetration of the signal will be reduced. A way to solve the issue of balancing between visualizing the surroundings and signal sensitivity is to superimpose images in real time by using separate cameras for reflectance and fluorescence. ${ }^{69,71}$

Kono et al..$^{56}$ performed the only comparative study to date with five laparoscopy systems for NIRF during cholecystectomy: Hamamatsu Photonics, Hamamatsu, Japan and Shinko Optical, Tokyo, Japan; Olympus Medical Systems, Tokyo, Japan; a high-definition unnamed model Japanese system; Karl Storz, Tuttlingen, Germany; Novadaq, Toronto, Canada. According to this study, the signal contrast was significantly different among the laparoscopic systems used for fluorescence imaging. In the meantime, since this study, systems and their properties have changed. The surgeon should be aware that differences exist which may influence the choice for a system.

Finally, it is important to evaluate the way the fluorescence intensity is assessed. Most studies only state whether a certain anatomical structure is visible or not, without quantitative measurement of the fluorescence intensity. There is no uniformly agreed analytic measure to objectify the fluorescence intensity. In most methods, only the fluorescence intensity of the target is measured, and not the fluorescence of the background.

The target-to-background ratio (TBR) is determined used by some authors (Schols et al. ${ }^{43,46}$, 
Ashitate et al. ${ }^{57}$, Kono et $a^{56}{ }^{56}$ Zarrinpar ${ }^{45}$, Verbeek ${ }^{48}$ ). Unfortunately, formulas that are used to calculate the ratio, the backgrounds chosen, and the software used for measurement of the signal are not uniformly. In practice, the absolute signal and the subjective observation by the surgeon is what determines the clinical usefulness of the NIRF image. But for research purposes and comparison between studies an objective, uniform and validated quantification is necessary, which, as illustrated by the differences described, is not available yet.

Our in vitro setup offers a simple and very cheap method for objective comparison between different system, but it would be recommendable that this topic is further refined and preferably standardized (e.g. by IEC).

The present review has some limitations. The majority of the studies are noncomparative. Especially to fulfil the purpose of the review, i.e. identify factors that influence the fluorescent image, such comparison would have been preferable. To partially address this drawback, ex vivo experiments were added to the review. In vivo studies would have been preferable, including the effect of human or animal tissue and circumstances on the acting of the fluorescent dye.

In conclusion, this study identified and discussed several factors that influence the signal of fluorescence cholangiography. Some are patient-specific and cannot be altered, such as BMI and cholecystitis. Even with a higher BMI and the presence of inflammation, NIRF is often successful. Other factors can be controlled by the surgeon. To maximize the fluorescence intensity during laparoscopic cholecystectomies, a weight-adjusted dose seems preferable over a fixed dose, especially in overweight patients; administration of this dose should take place as long as possible ( $\max 24 \mathrm{~h}$ ) before surgery; the laparoscope should be held at a right angle and close to the target tissue. Surgeons should be aware of new dyes that may come to the market and of differences in the properties of systems used, in order to benefit most of the potential of NIRF imaging. Further research should focus on the possibility of intra-gallbladder injection of ICG, other fluorescent dyes with a higher fluorescence intensity and faster clearance from the liver and the validation of a method to objectify the degree of fluorescence illumination in order to enable comparison between studies. 


\section{REFERENCES}

1. Statistiek CBvd (2010) Operaties in het ziekenhuis; soort opname, leeftijd en geslacht, 199520102010 [updated 05-02-2014; cited 2015]. Available from: http://statline.cbs.nl/StatWeb/ publication/?VW $=\mathrm{T} \& D M=S L N L \& P A=80386$ NED\&LA $=\mathrm{NL}$

2. Flum DR, Dellinger EP, Cheadle A, Chan L, Koepsell T (2003) Intraoperative cholangiography and risk of common bile duct injury during cholecystectomy. JAMA 289(13):1639-1644

3. Fletcher DR, Hobbs MS, Tan P, Valinsky LJ, Hockey RL, Pikora TJ et al (1999) Complications of cholecystectomy: risks of the laparoscopic approach and protective effects of operative cholangiography: a population-based study. Ann Surg 229(4):449-457

4. Nuzzo G, Giuliante F, Giovannini I, Ardito F, D'Acapito F, Vellone M et al (2005) Bile duct injury during laparoscopic cholecystectomy: results of an Italian national survey on 56591 cholecystectomies. Arch Surg 140(10):986-992

5. Waage A, Nilsson M (2006) latrogenic bile duct injury: a population-based study of 152776 cholecystectomies in the Swedish Inpatient Registry. Arch Surg 141(12):1207-1213

6. Booij KA, de Reuver PR, Yap K, van Dieren S, van Delden OM, Rauws EA et al (2015) Morbidity and mortality after minor bile duct injury following laparoscopic cholecystectomy. Endoscopy 47(1):40-46

7. Bobkiewicz A, Krokowicz L, Banasiewicz T, Koscinski T, Borejsza-Wysocki M, Ledwosinski W et al (2014) latrogenic bile duct injury. A significant surgical problem. Assessment of treatment outcomes in the department's own material. Pol Przegl Chir 86(12):576-583

8. Boerma D, Rauws EA, Keulemans YC, Bergman JJ, Obertop H, Huibregtse K et al (2001) Impaired quality of life 5 years after bile duct injury during laparoscopic cholecystectomy: a prospective analysis. Ann Surg 234(6):750-757

9. Landman MP, Feurer ID, Moore DE, Zaydfudim V, Pinson CW (2013) The long-term effect of bile duct injuries on healthrelated quality of life: a meta-analysis. HPB 15(4):252-259

10. Way LW, Stewart L, Gantert W, Liu K, Lee CM, Whang K et al (2003) Causes and prevention of laparoscopic bile duct injuries: analysis of 252 cases from a human factors and cognitive psychology perspective. Ann Surg 237(4):460-469

11. Nijssen MA, Schreinemakers JM, Meyer Z, van der Schelling GP, Crolla RM, Rijken AM (2015) Complications after laparoscopic cholecystectomy: a video evaluation study of whether the critical view of safety was reached. World J Surg 39(7):1798-1803

12. Buddingh KT, Nieuwenhuijs VB, van Buuren L, Hulscher JB, de Jong JS, van Dam GM (2011) Intraoperative assessment of biliary anatomy for prevention of bile duct injury: a review of current and future patient safety interventions. Surg Endosc 25(8):2449-2461

13. Dip FD, Asbun D, Rosales-Velderrain A, Lo Menzo E, Simpfendorfer CH, Szomstein S et al (2014) Cost analysis and effectiveness comparing the routine use of intraoperative fluorescent cholangiography with fluoroscopic cholangiogram in patients undergoing laparoscopic cholecystectomy. Surg Endosc 28(6):1838-1843

14. Ford JA, Soop M, Du J, Loveday BP, Rodgers M (2012) Systematic review of intraoperative cholangiography in cholecystectomy. Br J Surg 99(2):160-167

15. Schols RM, Connell NJ, Stassen LP (2015) Near-infrared fluorescence imaging for real-time intraoperative anatomical guidance in minimally invasive surgery: a systematic review of the literature. World J Surg 39(5):1069-1079

16. Verbeek FP, van der Vorst JR, Schaafsma BE, Hutteman M, Bonsing BA, van Leeuwen FW et al (2012) Image-guided hepatopancreatobiliary surgery using near-infrared fluorescent light. J Hepatobiliary Pancreat Sci 19(6):626-637

17. Mishra A, Behera RK, Behera PK, Mishra BK, Behera GB (2000) Cyanines during the 1990s: a review. Chem Rev 100(6):1973-20

18. Rodrigues EB, Meyer CH, Mennel S, Farah ME (2007) Mechanisms of intravitreal toxicity of indocyanine green dye: implications for chromovitrectomy. Retina 27(7):958-970 
19. Tagaya N, Shimoda M, Kato M, Nakagawa A, Abe A, Iwasaki Y et al (2010) Intraoperative exploration of biliary anatomy using fluorescence imaging of indocyanine green in experimental and clinical cholecystectomies. J Hepatobiliary Pancreat Sci 17(5):595-600

20. Cherrick GR, Stein SW, Leevy CM, Davidson CS (1960) Indocyanine green: observations on its physical properties, plasma decay, and hepatic extraction. J Clin Invest 39:592-600

21. Obana A, Miki T, Hayashi K, Takeda M, Kawamura A, Mutoh T et al (1994) Survey of complications of indocyanine green angiography in Japan. Am J Ophthalmol 118(6):749-753

22. Benya R, Quintana J, Brundage B (1989) Adverse reactions to indocyanine green: a case report and a review of the literature. Cathet Cardiovasc Diagn 17(4):231-233

23. Bjerregaard J, Pandia MP, Jaffe RA (2013) Occurrence of severe hypotension after indocyanine green injection during the intraoperative period. Case Rep 1(1):26-30

24. Schols RM, Lodewick TM, Bouvy ND, van Dam DA, Meijerink WJ, van Dam GM et al (2014) Near-infrared fluorescence laparoscopy of the cystic duct and artery in pigs: performance of a preclinical dye. J Laparoendosc Adv Surg Tech A 24(5):318-322

25. van den Bos J, Al-Taher M, Hsien SG, Bouvy ND, Stassen LP (2017) Near-infrared fluorescence laparoscopy of the cystic duct and cystic artery: first experience with two new preclinical dyes in a pig model. Surg Endosc 31(10):4309-4314

26. Srinivasan R, Singh M (2002) Development of biological tissue- equivalent phantoms for optical imaging. Indian J Exp Biol 40(5):531-535

27. Ballestriero R (2010) Anatomical models and wax venuses: art masterpieces or scientific craft works? J Anat 216(2):223-234

28. Diana M, Soler L, Agnus V, D’Urso A, Vix M, Dallemagne B et al (2017) Prospective Evaluation of precision multimodal gallbladder surgery navigation: virtual reality, near-infrared fluorescence, and x-ray-based intraoperative cholangiography. Ann Surg 266(5):890-897

29. Graves C, Ely S, Idowu O, Newton C, Kim S (2017) Direct gallbladder indocyanine green injection fluorescence cholangiography during laparoscopic cholecystectomy. J Laparoendosc Adv Surg Tech A 27(10):1069-1073

30. Boogerd LSF, Handgraaf HJM, Huurman VAL, Lam HD, Mieog JSD, van der Made WJ et al (2017) The best approach for laparoscopic fluorescence cholangiography: overview of the literature and optimization of dose and dosing time. Surg Innov 24(4):386-396

31. Ankersmit M, van Dam DA, van Rijswijk AS, van den Heuvel B, Tuynman JB, Meijerink W (2017) Fluorescent imaging with indocyanine green during laparoscopic cholecystectomy in patients at increased risk of bile duct injury. Surg Innov 24(3):245-252

32. Gangemi A, Danilkowicz R, Elli FE, Bianco F, Masrur M, Giulianotti PC (2017) Could ICG-aided robotic cholecystectomy reduce the rate of open conversion reported with laparoscopic approach? A head to head comparison of the largest single institution studies. J Robot Surg 11(1):77-82

33. Zroback C, Chow G, Meneghetti A, Warnock G, Meloche M, Chiu CJ et al (2016) Fluorescent cholangiography in laparoscopic cholecystectomy: the initial Canadian experience. Am J Surg 211(5):933-937

34. Igami T, Nojiri M, Shinohara K, Ebata T, Yokoyama Y, Sugawara G et al (2016) Clinical value and pitfalls of fluorescent cholangiography during single-incision laparoscopic cholecystectomy. Surg Today 46(12):1443-1450

35. Dip F, Nguyen D, Montorfano L, Noste ME, Lo Menzo E, Simpfendorfer C et al (2016) Accuracy of near infrared-guided surgery in morbidly obese subjects undergoing laparoscopic cholecystectomy. Obes Surg 26(3):525-530

36. van Dam DA, Ankersmit M, van de Ven P, van Rijswijk AS, Tuynman JB, Meijerink WJ (2015) Comparing near-infrared imaging with indocyanine green to conventional imaging during laparoscopic cholecystectomy: a prospective crossover study. J Laparoendosc Adv Surg Tech A 25(6):486-492 
37. Boni L, David G, Mangano A, Dionigi G, Rausei S, Spampatti S et al (2015) Clinical applications of indocyanine green (ICG) enhanced fluorescence in laparoscopic surgery. Surg Endosc 29(7):2046-2055

38. Dip F, Roy M, Lo Menzo E, Simpfendorfer C, Szomstein S, Rosenthal RJ (2015) Routine use of fluorescent incisionless cholangiography as a new imaging modality during laparoscopic cholecystectomy. Surg Endosc 29(6):1621-1626

39. Larsen SS, Schulze S, Bisgaard T (2014) Non-radiographic intraoperative fluorescent cholangiography is feasible. Dan Med J 61(8):A4891

40. Osayi SN, Wendling MR, Drosdeck JM, Chaudhry UI, Perry KA, Noria SF et al (2015) Near-infrared fluorescent cholangiography facilitates identification of biliary anatomy during laparoscopic cholecystectomy. Surg Endosc 29(2):368-375

41. Prevot F, Rebibo L, Cosse C, Browet F, Sabbagh C, Regimbeau JM (2014) Effectiveness of intraoperative cholangiography using indocyanine green (versus contrast fluid) for the correct assessment of extrahepatic bile ducts during day-case laparoscopic cholecystectomy. J Gastrointest Surg 18(8):1462-1468

42. Daskalaki D, Fernandes E, Wang X, Bianco FM, Elli EF, Ayloo S et al (2014) Indocyanine green (ICG) fluorescent cholangiography during robotic cholecystectomy: results of 184 consecutive cases in a single institution. Surg Innov 21(6):615-621

43. Schols RM, Bouvy ND, van Dam RM, Masclee AA, Dejong CH, Stassen LP (2013) Combined vascular and biliary fluorescence imaging in laparoscopic cholecystectomy. Surg Endosc 27(12):4511-4517

44. Buchs NC, Pugin F, Azagury DE, Jung M, Volonte F, Hagen ME et al (2013) Real-time nearinfrared fluorescent cholangiography could shorten operative time during robotic single-site cholecystectomy. Surg Endosc 27(10):3897-3901

45. Spinoglio G, Priora F, Bianchi PP, Lucido FS, Licciardello A, Maglione V et al (2013) Real-time near-infrared (NIR) fluorescent cholangiography in single-site robotic cholecystectomy (SSRC): a single-institutional prospective study. Surg Endosc 27(6):2156-2162

46. Schols RM, Bouvy ND, Masclee AA, van Dam RM, Dejong CH, Stassen LP (2013) Fluorescence cholangiography during laparoscopic cholecystectomy: a feasibility study on early biliary tract delineation. Surg Endosc 27(5):1530-1536

47. Kaneko J, Ishizawa T, Masuda K, Kawaguchi Y, Aoki T, Sakamoto Y et al (2012) Indocyanine green reinjection technique for use in fluorescent angiography concomitant with cholangiography during laparoscopic cholecystectomy. Surg Laparosc Endosc Percutan Tech 22(4):341-344

48. Buchs NC, Hagen ME, Pugin F, VolonteF, Bucher P, Schiffer Eetal (2012) Intra-operative fluorescent cholangiography using indocyanine green during robotic single site cholecystectomy. Int J Med Rob Comput Assist Surg 8(4):436-440

49. Ishizawa T, Kaneko J, Inoue Y, Takemura N, Seyama Y, Aoki T et al (2011) Application of fluorescent cholangiography to single-incision laparoscopic cholecystectomy. Surg Endosc 25(8):2631-2636

50. Ishizawa T, Bandai Y, ljichi M, Kaneko J, Hasegawa K, Kokudo N (2010) Fluorescent cholangiography illuminating the biliary tree during laparoscopic cholecystectomy. Br J Surg 97(9):1369-1377

51. Aoki T, Murakami M, Yasuda D, Shimizu Y, Kusano T, Matsuda K et al (2010) Intraoperative fluorescent imaging using indocyanine green for liver mapping and cholangiography. J Hepatobiliary Pancreat Sci 17(5):590-594

52. Mitsuhashi N, Kimura F, Shimizu H, Imamaki M, Yoshidome H, Ohtsuka M et al (2008) Usefulness of intraoperative fluorescence imaging to evaluate local anatomy in hepatobiliary surgery. $J$ Hepatobiliary Pancreat Surg 15(5):508-514

53. Liu YY, Liao CH, Diana M, Wang SY, Kong SH, Yeh CN et al (2017) Near-infrared cholecystocholangiography with direct intragallbladder indocyanine green injection: preliminary clinical results. Surg Endosc 32(3):1506-1514 
54. Zarrinpar A, Dutson EP, Mobley C, Busuttil RW, Lewis CE, Tillou A et al (2016) Intraoperative laparoscopic near-infrared fluorescence cholangiography to facilitate anatomical identification: when to give indocyanine green and how much. Surg Innov 23(4):360-365

55. Verbeek FP, Schaafsma BE, Tummers QR, van der Vorst JR, van der Made WJ, Baeten Cl et al (2014) Optimization of near-infrared fluorescence cholangiography for open and laparoscopic surgery. Surg Endosc 28(4):1076-1082

56. Kono Y, Ishizawa T, Tani K, Harada N, Kaneko J, Saiura A et al (2015) Techniques of fluorescence cholangiography during laparoscopic cholecystectomy for better delineation of the bile duct anatomy. Medicine 94(25):e1005

57. Ashitate Y, Stockdale A, Choi HS, Laurence RG, Frangioni JV (2012) Real-time simultaneous near-infrared fluorescence imaging of bile duct and arterial anatomy. J Surg Res 176(1):7-13

58. Landsman ML, Kwant G, Mook GA, Zijlstra WG (1976) Lightabsorbing properties, stability, and spectral stabilization of indocyanine green. J Appl Physiol 40(4):575-583

59. Hilderbrand SA, Weissleder R (2010) Near-infrared fluorescence: application to in vivo molecular imaging. Curr Opin Chem Biol 14(1):71-79

60. Frangioni JV (2003) In vivo near-infrared fluorescence imaging. Curr Opin Chem Biol 7(5):626634

61. Nguyen QT, Tsien RY (2013) Fluorescence-guided surgery with live molecular navigation-a new cutting edge. Nat Rev Cancer 13(9):653-662

62. Haritoglou C, Gandorfer A, Schaumberger M, Tadayoni R, Gandorfer A, Kampik A (2003) Lightabsorbing properties and osmolarity of indocyanine-green depending on concentration and solvent medium. Invest Ophthalmol Vis Sci 44(6):2722-2729

63. Matsui A, Tanaka E, Choi HS, Winer JH, Kianzad V, Gioux S et al (2010) Real-time intra-operative near-infrared fluorescence identification of the extrahepatic bile ducts using clinically available contrast agents. Surgery 148(1):87-95

64. Peter C, Hongwan D, Kupfer A, Lauterburg BH (2000) Pharmacokinetics and organ distribution of intravenous and oral methylene blue. Eur J Clin Pharmacol 56(3):247-250

65. van den Bos J, Schols RM, Luyer MD, van Dam RM, Vahrmeijer AL, Meijerink WJ et al (2016) Near-infrared fluorescence cholangiography assisted laparoscopic cholecystectomy versus conventional laparoscopic cholecystectomy (FALCON trial): study protocol for a multicentre randomised controlled trial. BMJ Open 6(8):e011668

66. Figueiredo JL, Siegel C, Nahrendorf M, Weissleder R (2010) Intraoperative near-infrared fluorescent cholangiography (NIRFC) in mouse models of bile duct injury. World J Surg 34(2):336-343

67. Liu YY, Kong SH, Diana M, Legner A, Wu CC, Kameyama N et al (2015) Near-infrared cholecystocholangiography with indocyanine green may secure cholecystectomy in difficult clinical situations: proof of the concept in a porcine model. Surg Endosc 30(9):4115-4123

68. Zhu B, Sevick-Muraca EM (2015) A review of performance of nearinfrared fluorescence imaging devices used in clinical studies. Br J Radiol 88(1045):20140547

69. AV DS, Lin H, Henderson ER, Samkoe KS, Pogue BW (2016) Review of fluorescence guided surgery systems: identification of key performance capabilities beyond indocyanine green imaging. J Biomed Opt 21(8):80901

70. Marshall MV, Rasmussen JC, Tan IC, Aldrich MB, Adams KE, Wang X et al (2010) Near-infrared fluorescence imaging in humans with indocyanine green: a review and update. Open Surg Oncol J 2(2):12-25

71. Orosco RK, Tsien RY, Nguyen QT (2013) Fluorescence imaging in surgery. IEEE Rev Biomed Eng 6:178-187 


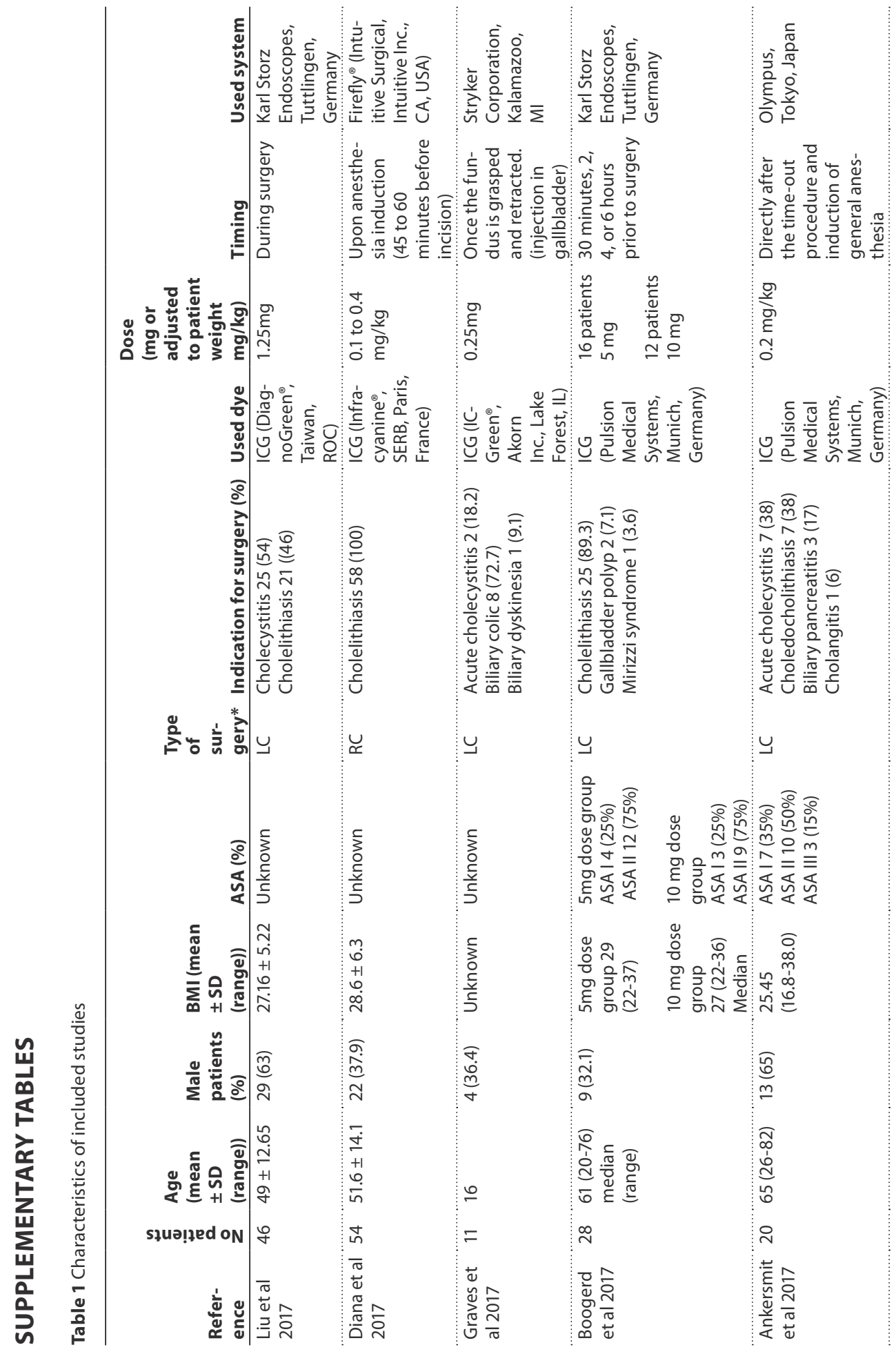




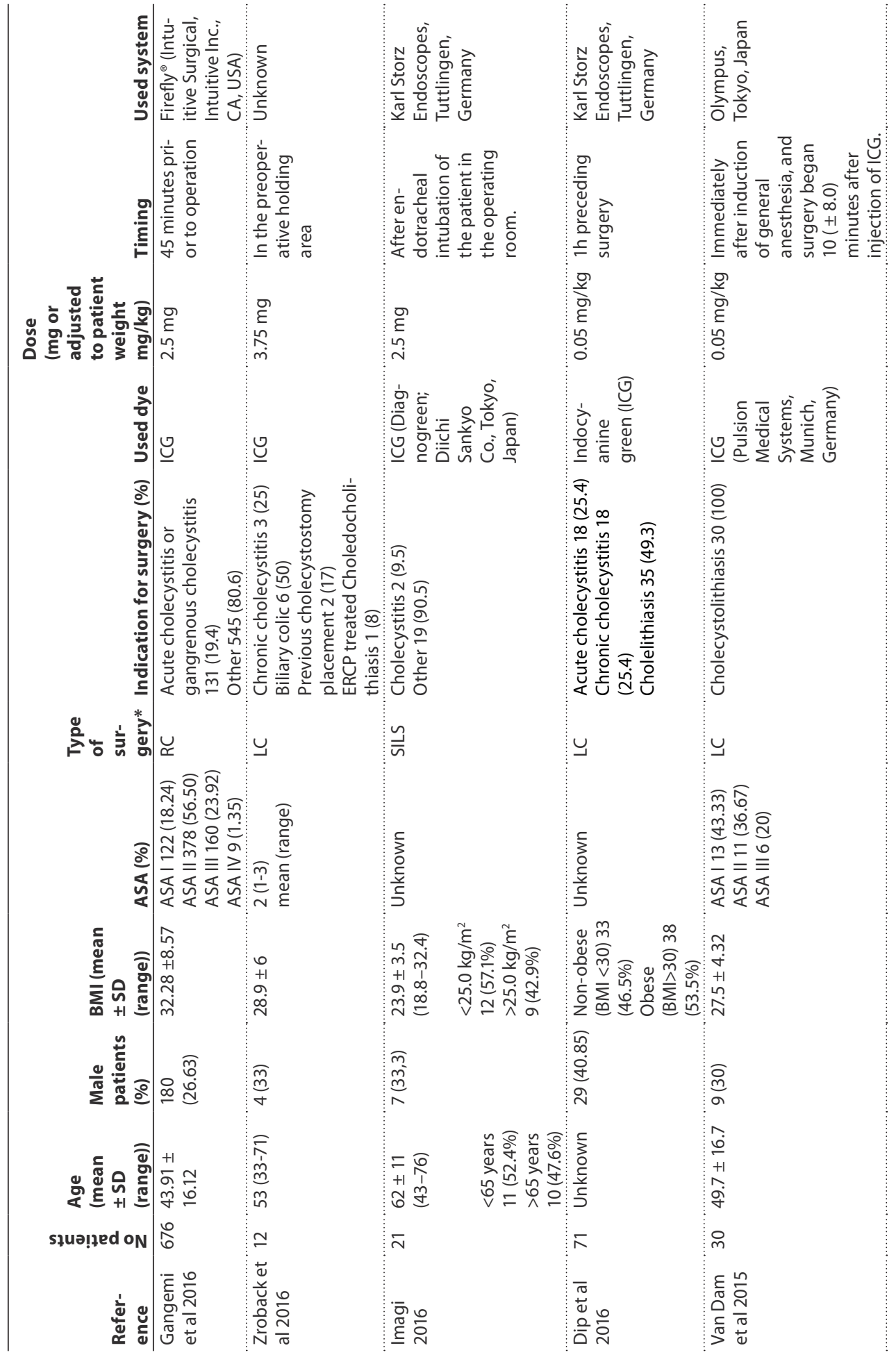




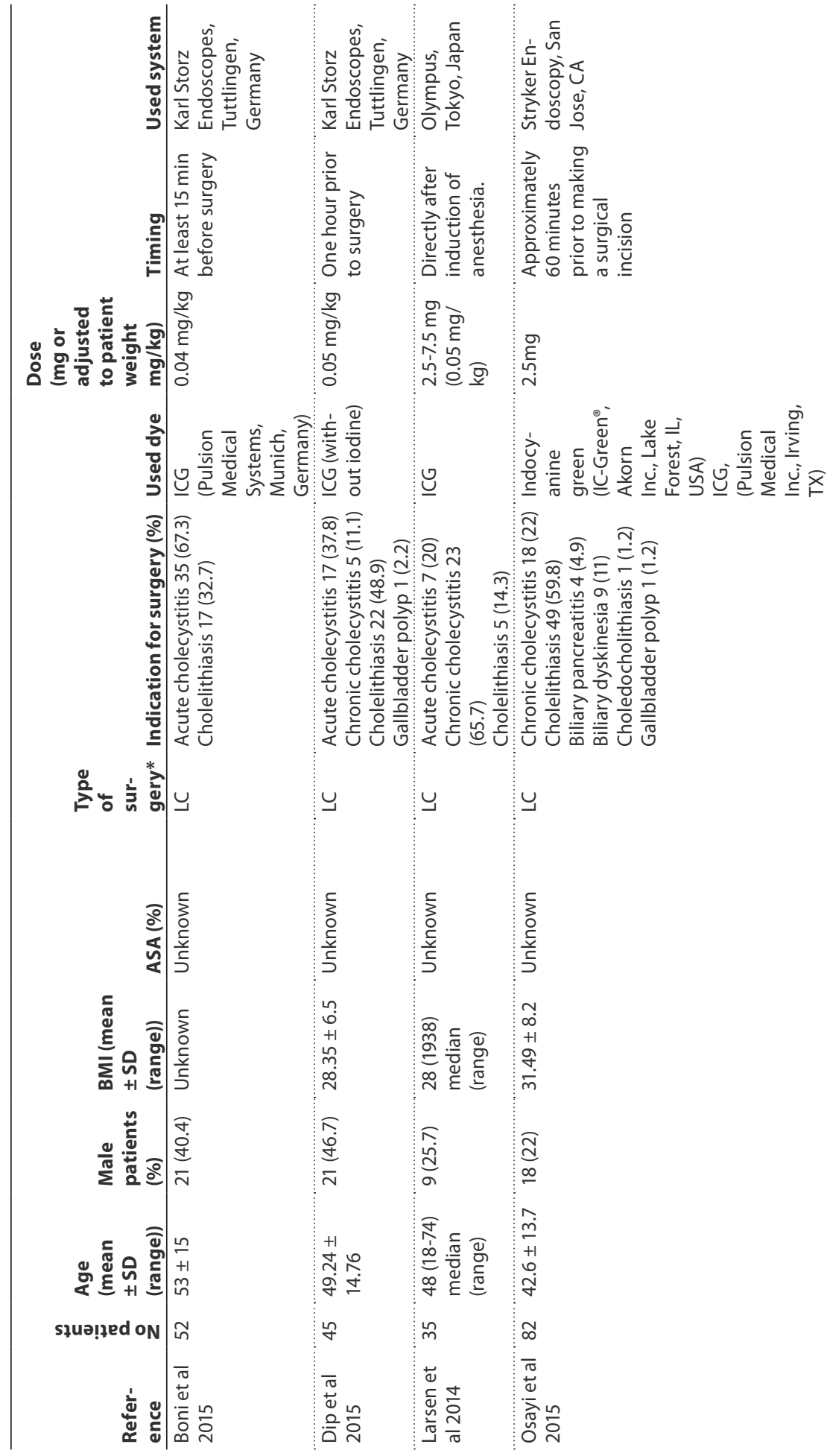




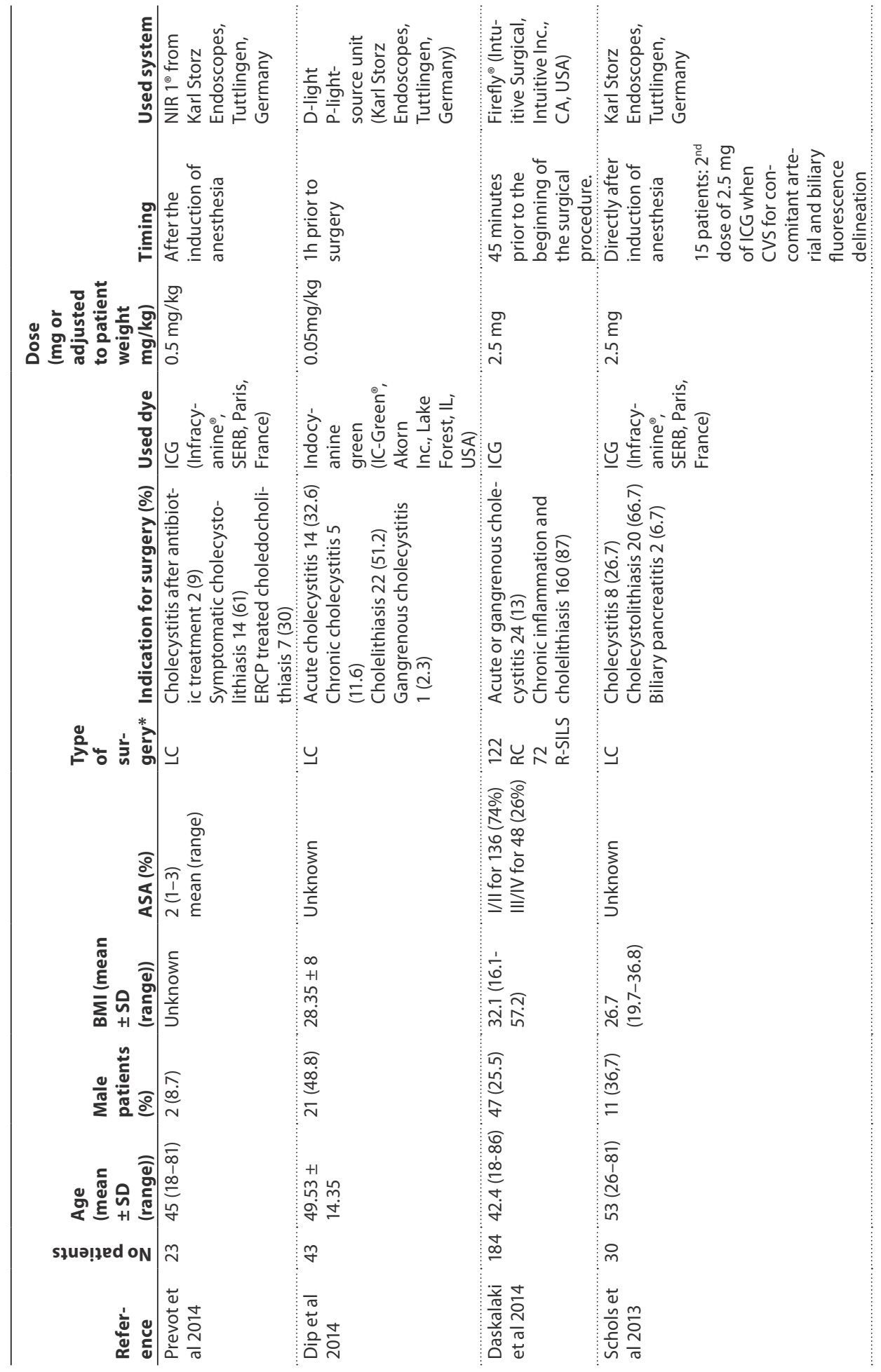




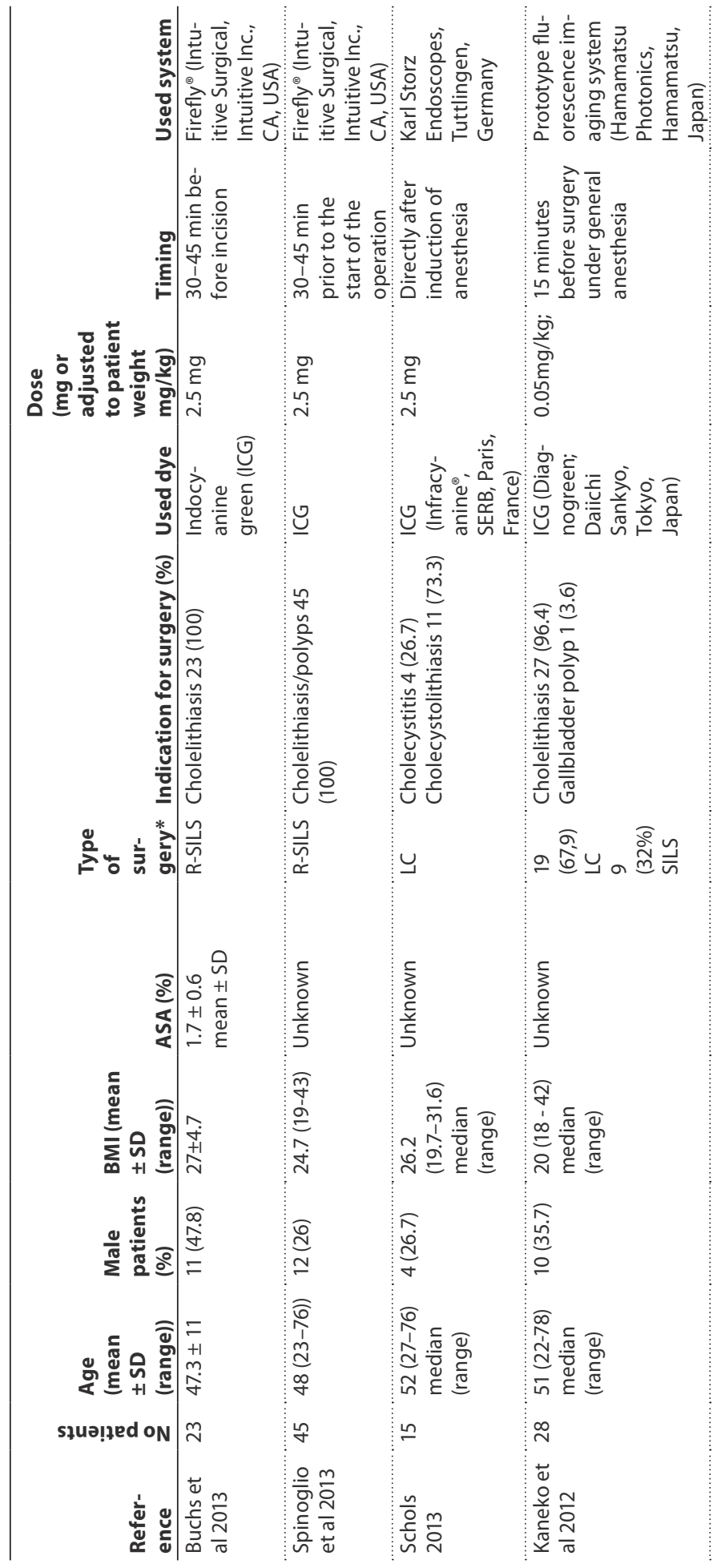




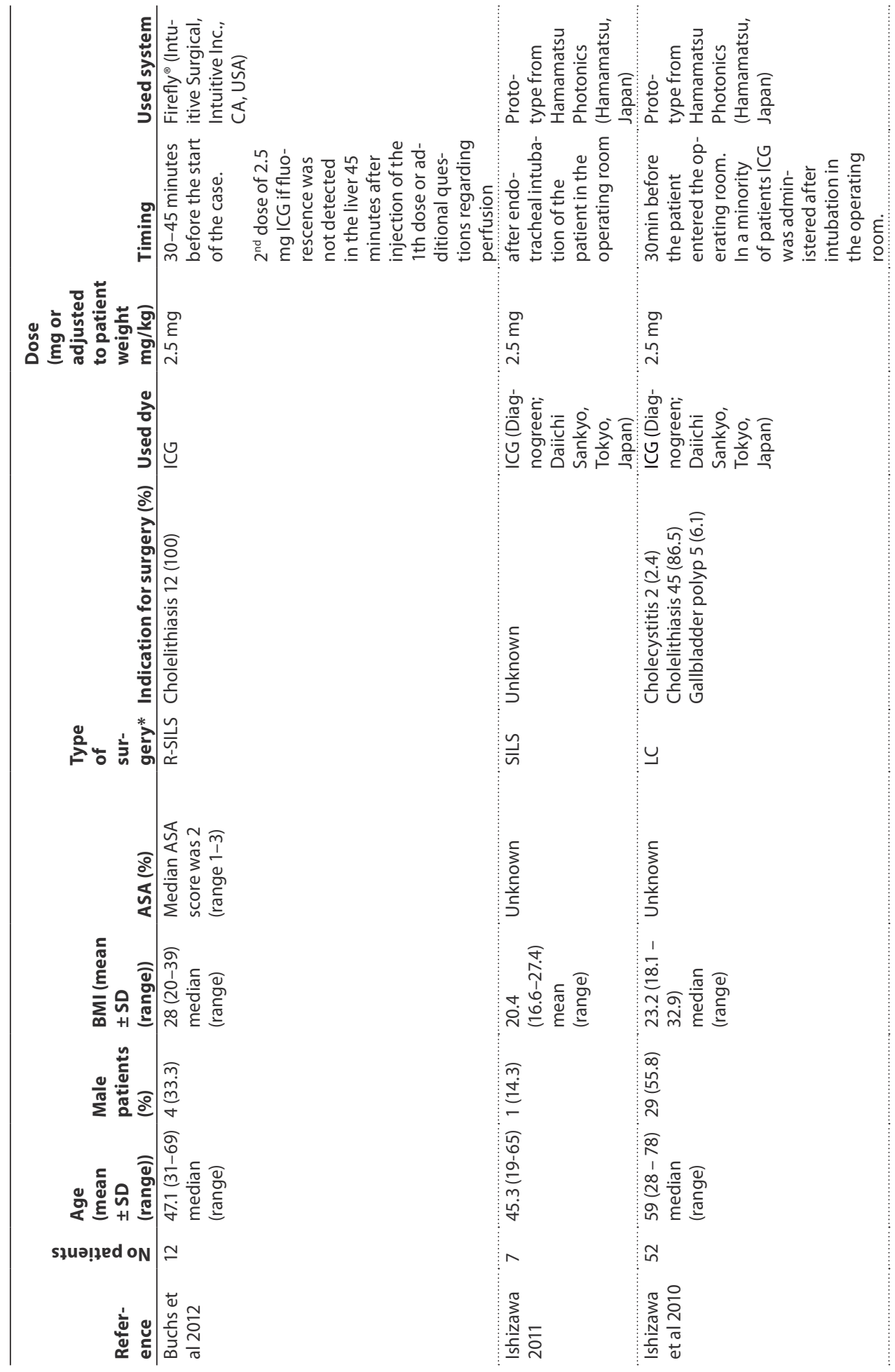




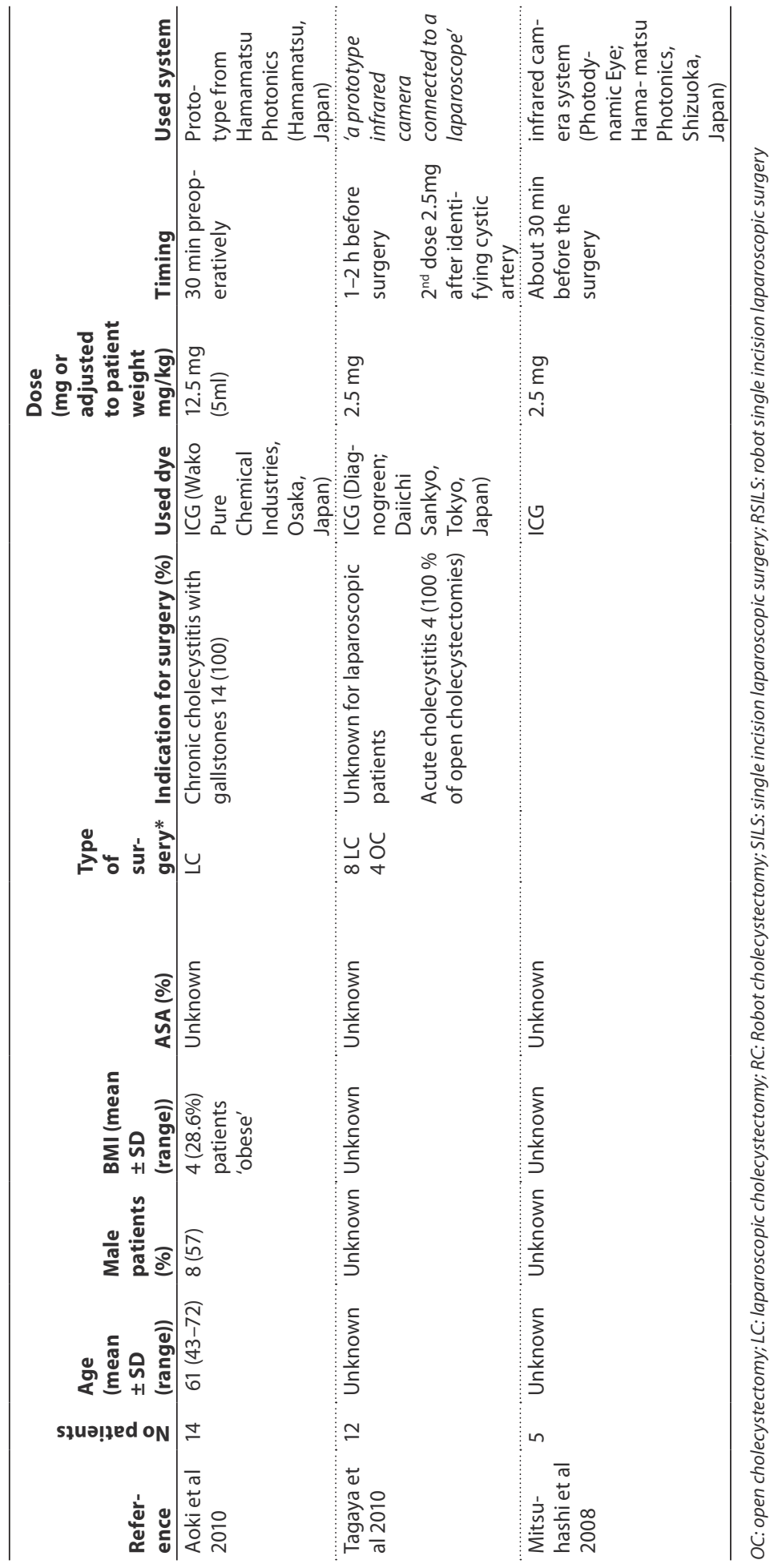




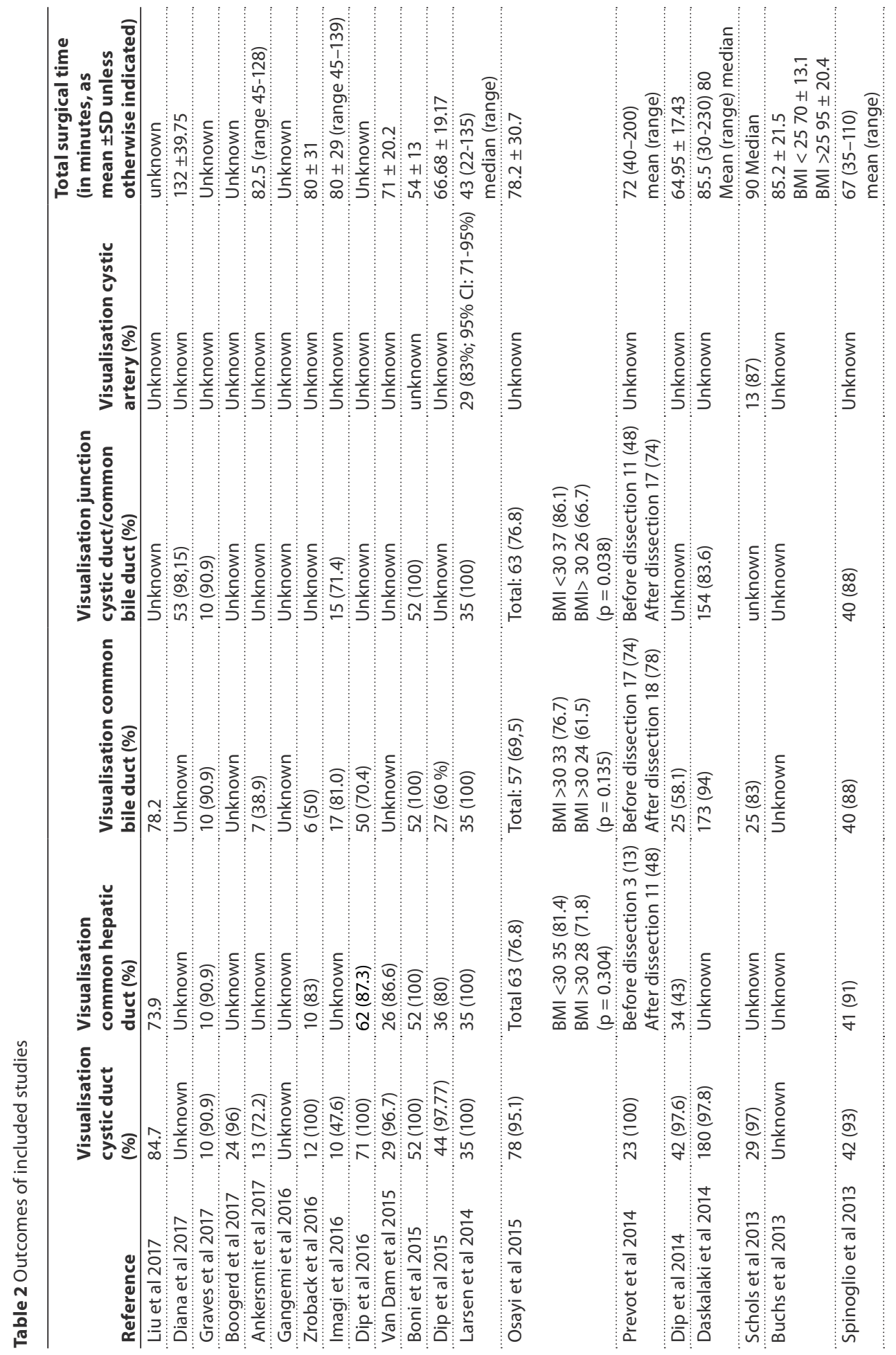




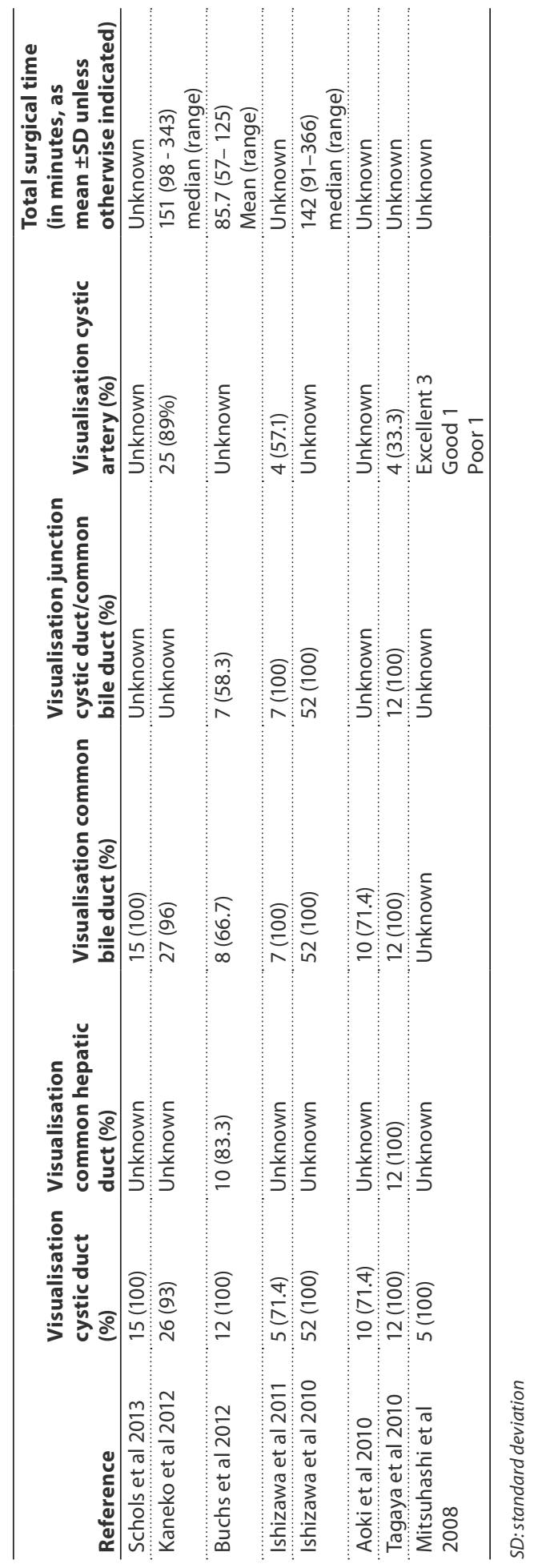


CHAPTER 4

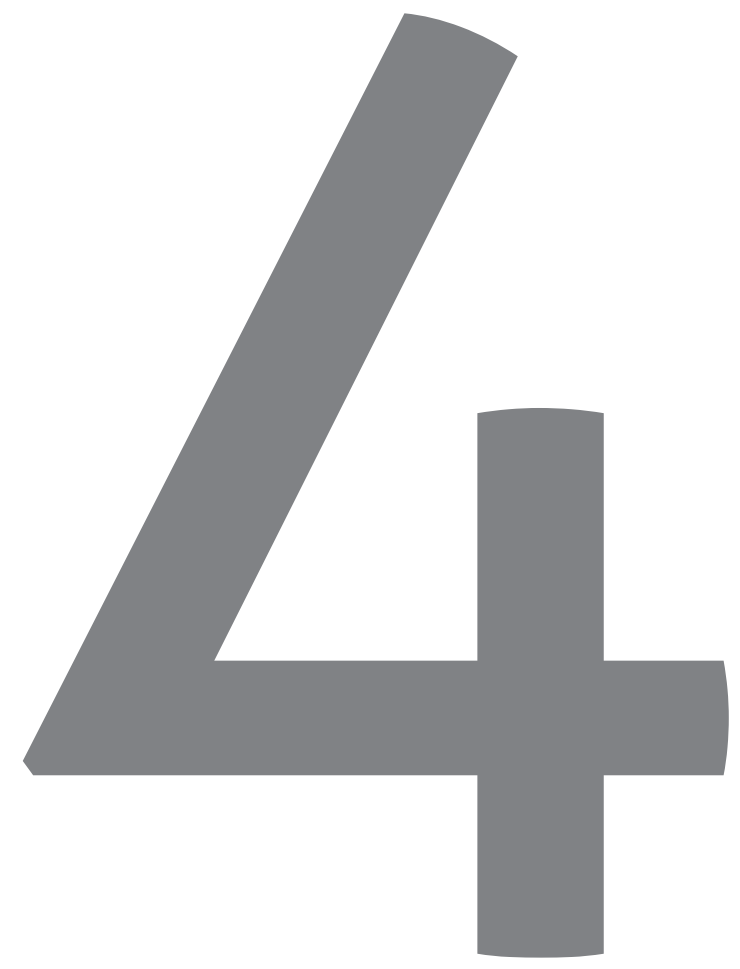




\section{Near infrared fluorescence laparoscopy of the cystic duct and cystic artery: first experience with two new preclinical dyes in a pig model}

Jacqueline van den Bos, Mahdi Al-Taher, Shu Gi Hsien, Nicole D. Bouvy, Laurents P.S. Stassen.

Surgical Endoscopy 2017;31(10):4309-14 


\section{ABSTRACT}

\section{Background}

Imaging techniques that enhance visualisation of the anatomy may help prevent bile duct injury. Near-Infrared Fluorescence Imaging is such a technique. Previous experiments with ICG have shown that illumination of the extra-hepatic bile ducts is feasible. Yet, there is room for improvement in the visualisation of the target as compared to the background. Experiments with IRDye ${ }^{\circledR} 800 \mathrm{CW}$ show promising results. However, this dye is too expensive for routine clinical use. The aim of this study is to test the first applicability of two newly developed preclinical dyes regarding intraoperative imaging of the cystic duct and cystic artery, compared with IRDye ${ }^{\circledR} 800 \mathrm{CW}$.

\section{Methods}

Laparoscopic cholecystectomy was performed in three pigs, using a laparoscopic fluorescence imaging system. Each pig received $6 \mathrm{mg}$ of one of the fluorescent dyes ( $1 \mathrm{mg} / \mathrm{mL}$; IRDye ${ }^{\oplus} 800 \mathrm{CW}$, IRDye ${ }^{\circledast} 800 \mathrm{BK}$ or IRDye ${ }^{\oplus} 800 \mathrm{NOS}$ ) by intravenous injection. Intraoperative recognition of the biliary system and cystic artery was registered at set time points. All procedures were digitally recorded, and the target to background ratio (TBR) was determined to assess the fluorescence signal.

\section{Results}

With all three fluorescent dyes, the cystic artery was directly visualized. For the visualization of the cystic duct, 15, 34 and 30 min were needed using IRDye ${ }^{\circledR} 800 B K$, IRDye $^{\circledR} 800$ NOS and IRDye ${ }^{\circledR} 800 \mathrm{CW}$, respectively. The maximum TBR of the cystic duct was the highest with IRDye ${ }^{\circledast} 800$ NOS (4.20) after 36 min, compared to 2.45 for IRDye ${ }^{\circledast} 800$ BK and 2.15 for IRDye ${ }^{\circledR}$ $800 \mathrm{CW}$, both after $45 \mathrm{~min}$. There were no adverse events.

\section{Conclusion}

IRDye $^{\circledR} 800$ BK and IRDye ${ }^{\circledast} 800$ NOS seem to be good alternatives for IRDye ${ }^{\circledR} 800 C W$ for the visualization of the cystic duct and cystic artery in pigs. 


\section{INTRODUCTION}

Laparoscopic cholecystectomy is standard care for symptomatic gallbladder stones or acute cholecystitis and is one of the most commonly performed surgical procedures in the Western hemisphere. The reported incidence of bile duct injuries in laparoscopic surgery is 0.4-1.4\%., 2 Such injuries are associated with increased morbidity, reduced survival, impaired quality of life and have economic consequences for society. ${ }^{2,3}$ Anatomical variations of the biliary ducts or vascular system are not uncommon and represent operative challenges and looming sources for operative complications., 4 Therefore, enhanced intraoperative visualization of the biliary anatomy is needed to reduce the incidence of bile duct injuries. Near-infrared fluorescence (NIRF) imaging is a promising technique for real-time delineation of the perioperative anatomy. Among other applications, it is used for visualization of the extra-hepatic bile ducts and the cystic artery in laparoscopic cholecystectomy, and visualization of the ureter in colorectal, urological and gynecological surgery. ${ }^{5-7}$ In the clinical setting, ICG is the dye used for bile duct imaging, with reasonable visualization results that makes clinical use possible. Both the extra-hepatic bile ducts and the cystic artery can be identified. ${ }^{8}$ Subjectively however, imaging of the target is not optimal in all patients. Better contrast between target and background is expected to increase the usefulness of the technique. Adaptation in the equipment and its characteristics is one way to address this issue. Alteration in the dye or its use is another. One such alteration is the suggestion to inject the dye a day before surgery. ${ }^{9}$ However, due to usually performed same-day admission, this is undesirable from a logistic standpoint. Applying another fluorescent dye is a further possibility.

IRDye ${ }^{\circledR} 800 C W$ (LICOR Biotechnology, Lincoln, United States) is an experimental dye, which in combination with laparoscopic imaging allows intraoperative visualization of crucial anatomical structures. In a previous animal experiment, performed by our group, this dye was compared to ICG for imaging of the extra-hepatic bile ducts and the cystic artery in the pig. The main results were an earlier visualization of the cystic duct, when using IRDye ${ }^{\oplus} \mathrm{CW}-800$ and a higher target-to-background ratio for the cystic artery. ${ }^{10}$ This is in line with two other studies and makes this new dye promising for future clinical use..$^{10-12}$ However, a major disadvantage of IRDye ${ }^{\circledR} \mathrm{CW}$-800 is its cost, which is almost tenfold that of ICG. Therefore, more affordable alternatives are needed.

Recently, the manufacturer of IRDye ${ }^{\circledast} 800 \mathrm{CW}$ developed two new preclinical dyes, IRDye $^{\circledast} 800$ BK and IRDye ${ }^{\oplus} 800$ NOS, that can be produced at a cost that is comparable to commercially available ICG. In general, the three dyes have similar properties. Emission and absorption are at about the same wavelength as for ICG. The dyes differ in hydrophilic properties, with IRDye ${ }^{\circledast} 800$ NOS being the least hydrophilic. This influences their excretion and their uptake in the liver and may make them more potent with regard to their imaging capabilities of the cystic duct. 
The aim of the present study was to evaluate the performance of these dyes as compared to IRDye ${ }^{\circledR} 800 \mathrm{CW}$ for NIRF laparoscopy of the extra-hepatic bile ducts and the cystic artery in pigs using a commercially available laparoscopic fluorescence imaging system.

\section{MATERIALS AND METHODS}

This study was conducted at the central animal facilities of Maastricht University (Maastricht, The Netherlands). Animals were used in compliance with the regulations of the Dutch legislation for animal research and following a protocol that was approved by the local animal ethics committee. A pig model was chosen because of its similarity with human hepatobiliary anatomy and because previously successful experiments have been conducted with NIRF imaging in pigs. ${ }^{10}$ Three female Dutch landrace pigs, weighing $40 \mathrm{~kg}$, were used for the current study. After surgery, the pigs were sacrificed by the anesthesiologist.

\section{Laparoscopic fluorescence imaging system}

A commercially available laparoscopic fluorescence imaging system (Karl Storz GmbH \& CO. KG, Tuttlingen, Germany) was used. The NIR light source D-Light $P$ is a xenon-based system providing illumination light for fluorescence applications in the near-infrared wavelength range. It enables the excitation of all presented dyes: IRDye 800CW (KEX/EM $=775 / 796 \mathrm{~nm})$, IRDye 800BK (KEX/EM =774/790 nm) and IRDye 800NOS (KEX/EM =767/786 nm). A foot pedal allows the surgeon to easily switch between the two imaging modalities. All procedures were digitally recorded. For all three dyes, the same NIRF imaging settings were used.

\section{Characteristics and preparation of near-infrared dyes}

IRDye ${ }^{\circledR} 800 \mathrm{CW}$ is a tetrasulphonated heptamethine indocyanine dye. Intravenous injection is rapidly cleared by the liver and excreted into bile. It is also cleared by the kidneys and excreted into urine. The peak absorption of this dye is $775 \mathrm{~nm}$ and peak excitation emission at $796 \mathrm{~nm}$. The molecular weight is $1090.11 \mathrm{Da}{ }^{13,14}$

IRDye ${ }^{\oplus} 800$ BK (LICOR Biotechnology, Lincoln, United States) is a newly developed dye, primarily developed for intraoperative visualization of the ureters. This hydrophilic dye has a maximum absorption of $774 \mathrm{~nm}$ and a maximum emission of $790 \mathrm{~nm}$. Because of its hydrophilic nature, it is primarily cleared by the kidneys. Nevertheless, being similar in structure as IRDye ${ }^{\oplus} 800 \mathrm{CW}$, some additional clearance by the liver can be expected.

IRDye ${ }^{\circledast} 800$ NOS (LICOR Biotechnology, Lincoln, United States) is also a newly developed dye, less hydrophilic, mainly developed for intraoperative visualization of the biliary system. This dye is primarily cleared by the liver and has a maximum absorption of $767 \mathrm{~nm}$ and a maximum emission of $786 \mathrm{~nm}$. 
All three dyes were prepared following instructions of the manufacturer. They were diluted in a sterile phosphate buffered saline (PBS) solution to a concentration of 1 milligram per milliliter. Six milligrams of each dye in this dilution was prepared for intravenous injection.

\section{Surgical technique}

Premedication consisted of intramuscular injection of azaperone $3 \mathrm{mg} / \mathrm{kg}$, ketamine $10 \mathrm{mg} /$ $\mathrm{kg}$ and atropine $0.05 \mathrm{mg} / \mathrm{kg}$. Anesthesia was induced with intravenous thiopental $10-15 \mathrm{mg} /$ $\mathrm{kg}$. After intubation, the pigs were maintained under anesthesia with isoflurane and oxygen. Two expert endoscopic gastrointestinal surgeons (NB and LS) performed the operations and were assisted by residents experienced in the procedure. In each pig, a laparoscopic cholecystectomy was performed, according to the Dutch Guidelines and best practice for laparoscopic cholecystectomy applying the Critical View of Safety technique. ${ }^{15,16}$ One dye was tested per animal using a $6 \mathrm{mg}$ dose $(1 \mathrm{mg} / \mathrm{mL})$. This was twice the dose proven sufficient in an earlier study. ${ }^{10}$ The dose was chosen to obtain maximal visualization since our hypothesis was that a higher dose of the dye would result in better biliary imaging. Fluorescence imaging was initiated immediately after dye injection and subsequently imaging was performed intermittently in fluorescence mode and white light mode. Intraoperatively, the researcher systematically documented on a form whether the cystic duct or cystic artery could be identified, in either of the imaging modes. For agreement on the identification on the aforementioned structures, the attending surgeon was consulted. A structure was scored as 'identified' if its localization was confirmed with great certainty by the experienced surgeon.

\section{Postoperative quantitative fluorescence analysis}

The video recordings of the procedures were assessed for the degree of fluorescence illumination using OSIRIX v7.0.1 Imaging software (Pixmeo, Geneva, Switzerland). With this software, the Target-to-Background Ratio (TBR) could be determined. The TBR was defined as the mean fluorescence intensity (Fl in arbitrary units, A.U.) of three points of interest in the target (cystic artery or cystic duct), minus the mean fluorescence intensity of three points of interest in the background (liver hilum), divided by the mean fluorescence intensity of three points of interest in the background. In formula: TBR $=$ (FI of target - FI of background)/ (FI background). ${ }^{8,10}$ Areas with light scattering were avoided in these points of interest.

\section{RESULTS}

In all experiments, the identification of the cystic duct and cystic artery with NIRF imaging was successfully conducted. Results obtained during the operation are presented in the intraoperative registration form in Table 1. 
Table 1 Intraoperative registration form

\begin{tabular}{|c|c|c|c|c|}
\hline Experiment & $\begin{array}{l}\text { Injected dye } \\
(6 \mathrm{mg})\end{array}$ & $\begin{array}{l}\text { Visualization of } \\
\text { cystic artery? }\end{array}$ & $\begin{array}{l}\text { Visualisation of } \\
\text { cystic duct? }\end{array}$ & $\begin{array}{l}\text { Time to } \\
\text { identification } \\
\text { cystic duct }\end{array}$ \\
\hline 1 & IRDye ${ }^{\circledR} 800 \mathrm{BK}$ & Yes & Yes & 15 minutes \\
\hline 2 & IRDye ${ }^{\circledast} 800$ NOS & Yes & Yes & 34 minutes \\
\hline 3 & IRDye $^{\oplus} 800 \mathrm{CW}$ & Yes & Yes & 30 minutes \\
\hline
\end{tabular}

In the first pig (IRDye $\left.{ }^{\circledast} 800 \mathrm{BK}\right)$, direct clear visualization of the cystic artery was present after injecting the fluorescent dye. Already after $15 \mathrm{~min}$, the cystic duct showed NIRF signal. The second pig (IRDye ${ }^{\circledast}$ 800NOS) showed immediate visualization of the cystic artery after injection of the dye. However, due to a technical problem early in the procedure, this visualization was not recorded, and therefore no TBR could be obtained from this fluorescent cystic artery. After $34 \mathrm{~min}$, the cystic duct was clearly visible. However, manipulation of the gallbladder proved to be of influence on the visualization of the cystic duct. Too much stretching of the fragile duct prohibited influx of fluorescent dye. This was corrected at 34 min. As in the first two pigs, the cystic artery was also clearly visible in the third pig receiving IRDye ${ }^{\oplus} 800 \mathrm{CW}$ dye. After $30 \mathrm{~min}$, the cystic duct could be identified in the fluorescence mode. In all three pigs, apart from the cystic duct and cystic artery, other structures became visible. The bicornate uterus was subjectively the most fluorescent in the abdomen of all three pigs. Also, the ureters, small bowel and lymph nodes were visible in fluorescence mode in all three pigs.

\section{Target-to-background ratio}

Postoperatively, the video recordings were analyzed by measuring the Target-to-Background Ratio (TBR). The TBR's of cystic artery for IRDye ${ }^{\circledR} 800 B K$ and IRDye ${ }^{\oplus} 800 C W$ were 6.78 and 7.54 , respectively. The TBR of the cystic artery for IRDye ${ }^{\circledast} 800$ NOS could not be measured because of a technical problem regarding the video recording procedures early in the operation. The maximum measured TBRs from the three dyes for the cystic duct were 2.45 (at $45 \mathrm{~min}$ ), 4.20 (at $35 \mathrm{~min}$ ) and 2.15 (at $45 \mathrm{~min}$ ) for IRDye ${ }^{\circledR}$ 800BK, IRDye ${ }^{\circledR} 800 \mathrm{NOS}$ and IRDye ${ }^{\circledR}$ $800 \mathrm{CW}$, respectively. Images 1-3 show the cystic ducts during these maximum measured TBRs. All images were made in the same fluorescence settings of the Image1 SPIES system. However, as can be seen in the images, with the IRDye ${ }^{\oplus} 800$ NOS, the laparoscope was at further distance from the cystic duct. The images with the maximum TBR for each dye are shown in Fig. 1, 2 and 3.

\section{Safety}

In none of the pigs complications or adverse reactions were registered during surgery that could be attributed to one of the dyes. In particular, no alteration in heart rate and 


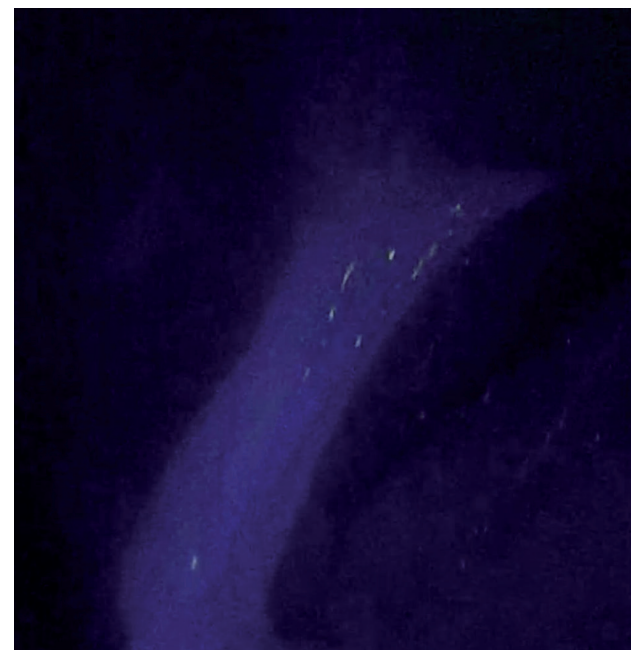

Figure 1 Visualization of the cystic duct, $45 \mathrm{~min}$ (time point of maximal signal) after injecting $6 \mathrm{mg}$ of IRDye ${ }^{\circledR} 800 \mathrm{BK}(1 \mathrm{mg} / \mathrm{mL})$.

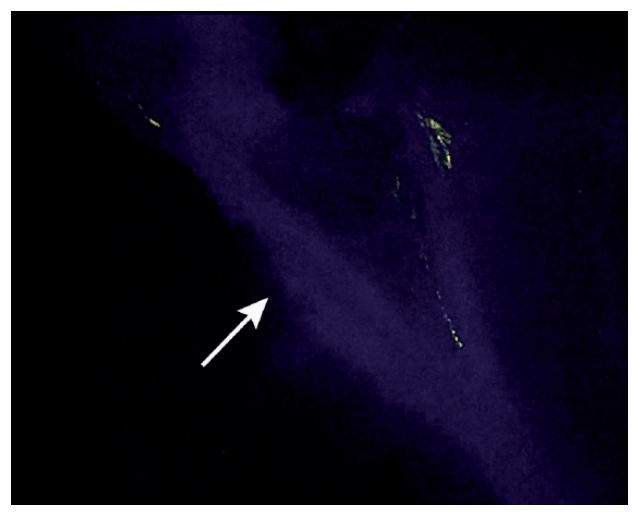

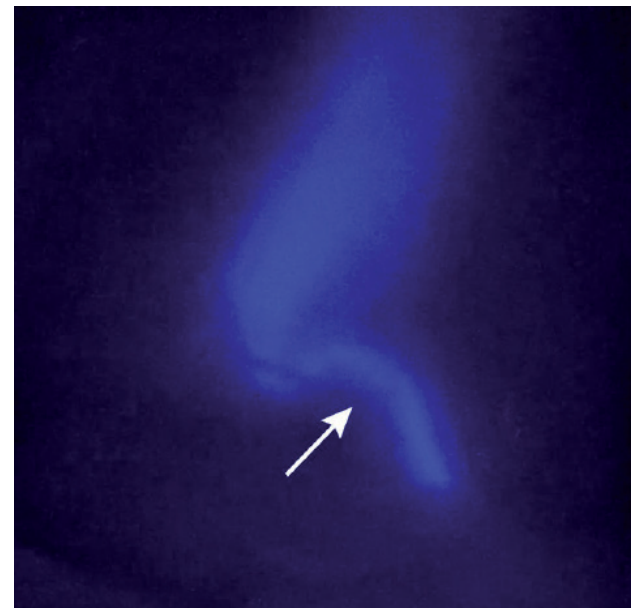

Figure 2 Visualization of the cystic duct (pointed by arrow), and the base of the gallbladder $35 \mathrm{~min}$ (time point of maximal signal) after injecting $6 \mathrm{mg}$ of IRDye ${ }^{\circledR} 800 \mathrm{NOS}(1 \mathrm{mg} / \mathrm{mL})$

Figure 3 Visualization of the cystic duct (pointed by arrow) and common bile duct, 45 min (time point of maximal signal) after injecting $6 \mathrm{mg}$ of IRDye ${ }^{\circledR}$ $800 \mathrm{CW}(1 \mathrm{mg} / \mathrm{mL})$.

Note: the little white spots are caused by scattering, and do not represent the fluorescent signal

blood pressure of respiratory status was observed. Only a slightly and transient decrease in intraoperative monitoring of the Saturation of peripheral Oxygen (SpO2) was observed during the first minute after injection of the fluorescent dyes.

\section{DISCUSSION}

In the present study, the use of two new preclinical dyes for NIRF imaging of the extrahepatic bile ducts with a commercially available laparoscopic imaging system was investigated. The new dyes resemble the structure and spectral characteristics of the previously favorably tested IRDye ${ }^{\circledast} 800 \mathrm{CW}$ but are much less costly, now being in the range of commercially available ICG. Both features are essential for possible future clinical application. The aim of 
the experiments was to perform a first assessment of the NIRF properties of the new dyes and compare these to the performance of IRDye ${ }^{\circledR} 800 \mathrm{CW}$. Eventually, these dyes could be an alternative to ICG, the only dye presently available for human use in NIRF imaging of the biliary system. This would be desirable, as with ICG, although NIRF visualization of the bile ducts can be achieved, the observed contrast of target-to-background leaves room for improvement.

Other advantages are a shorter period between injection of the dye and visualization of the target structures and the fact that these new dyes contain no iodine, whereas some of the commercially available ICG preparations do. ${ }^{17}$ These new dyes are therefore also applicable in patients that are known with an iodine allergy or hypersensitivity. A final benefit could be that these dyes are not only exclusively cleared by the liver, but also by the kidneys, a fact that is known from IRDye ${ }^{\oplus} 800 \mathrm{CW}$. This may make these dyes appropriate for ureter imaging.

All three dyes showed clear visualization of the cystic artery directly after intravenous injection. Between 15 and 35 minutes, with all three dyes, clear visualization of the cystic duct was achieved. Both IRDye ${ }^{\circledast} 800 B K$ and IRDye ${ }^{\circledast} 800$ NOS caused a more prominent fluorescent signal than the earlier tested IRDye ${ }^{\circledR} 800 \mathrm{CW}$. The TBR of 2.15 found for IRDye ${ }^{\circledR}$ $800 \mathrm{CW}$ in this study is comparable with that in an earlier study, where the TBR was $2.3 .^{10}$ The signal from IRDye ${ }^{\circledast} 800$ NOS was stronger than that from IRDye ${ }^{\circledast 800 B K}$. This was expected, as IRDye ${ }^{\circledast} 800$ NOS is less hydrophilic and supposed to be excreted through the bile more exclusively than IRDye ${ }^{\circledR} 800$ BK. In a previous study, the TBR's of ICG and IRDye ${ }^{\oplus}$ CW800 were comparable. ${ }^{10}$ The present results suggest that the IRDye ${ }^{\circledast} 800$ NOS might be more potent for bile duct imaging. This corresponds to the subjective impression of the surgeons. Of course, these data have to be interpreted with caution and need confirmation. A possible limitation in the measurement of the TBR in this study is the not uniformly defined distance between laparoscope and cystic duct at varying time points. As shown in Images 1-3, this distance is greater in the pig with IRDye ${ }^{\oplus} 800 \mathrm{NOS}$. A larger distance between the region of interest and the laparoscope may lead to a lower fluorescence intensity. ${ }^{18}$ However, the TBR for IRDye ${ }^{\oplus} 800$ NOS was the highest of all found TBRs.

There were no adverse reactions as a result of the administration of the dye. A very early and transient decrease in oxygen saturation was measured; this is a common phenomenon with the use of intravenous fluorescent dyes. ${ }^{19-21}$ The transient changing of the color of the blood results in the oxygen measurements becoming falsely lower than the real value.

During the experiment, it was observed that the three dyes were all cleared by both the liver (and excreted into bile) and the kidneys (and excreted into urine). Therefore, all three dyes might be eligible for both imaging of the biliary anatomy and of the ureters, although, as previously mentioned, IRDye ${ }^{\circledast} 800$ NOS is more exclusively excreted in bile and IRDye ${ }^{\circledast} 800 B K$ more in urine. This was confirmed by the higher TBR in the bile duct for IRDye ${ }^{\circledR}$ 800 NOS in this experiment. The exact behavior of these dyes needs further study for both 
biliary and urinary imaging. Because of the uncertainty on the amount of the dyes that is lost in urine, the given dose was set twice as high as in a previous experiment with successful bile duct imaging with IRDye ${ }^{\varpi} 800 \mathrm{CW}^{10}{ }^{10}$ The TBR of IRDye ${ }^{\circledast} 800 \mathrm{CW}$ in the present study was comparable to the previous observation which might indicate that the double dose is of no influence when using IRDye ${ }^{\oplus} 800 \mathrm{CW}$. In the present study, IRDye ${ }^{\oplus} 800 \mathrm{CW}$ took longer to become visible than reported in the study by Schols et al. 2014, where the cystic duct was distinguishable after 11 min. ${ }^{10}$ Tanaka et al. 2014 measured fluorescence in the cystic duct after around 20 min, which is comparable to the current results. ${ }^{11}$ The different doses used may have had an influence on the time until the appearance of fluorescence. Neither can it be ruled out that another dose than the one chosen gives superior imaging. Further experiments are needed to determine optimal dosing and timing that are influenced by the pharmacokinetic properties of the dyes.

This study has two other limitations. First, because of the limited series of experiments, the results have to be interpreted with caution. Since only three pigs were used, each dye was tested only in one pig. This resulted in the fact that the failure to start video recordings early in the second pig, in which IRDye ${ }^{\circledast} 800$ NOS was tested, could not be corrected in a subsequent experiment. Measurement of the visualization of the cystic artery with TBR was therefore not possible in this pig. Another limitation is the fact that we observed influence of the manipulation with the gallbladder and cystic duct on flow of the fluorescent dye in the bile through the cystic duct in the second pig. It cannot be ruled out that earlier imaging could have been obtained with adaptation of manipulation. This may have influenced the bile flow and the detailed time measurements in the other pigs. A drawback of the procedures was the necessity to switch intermittently between NIRF- and white light imaging of the biliary system. This is inherent to the equipment used. Although likely that simultaneous white- and fluorescent light imaging is more practical, the surgeons did not experience this as hindering, and measurements could be performed at a sufficient number of time points to perform relevant observations.

This study focusses fully on the evaluation of new dyes to optimize NIRF imaging. Another way to improve NIRF imaging is adaptation of the equipment and its properties. Such approach is also very important, but outside the scope of the present experiments.

\section{CONCLUSION}

The present study shows promising results on two new and affordable dyes for NIRF imaging: IRDye ${ }^{\circledast} 800 \mathrm{BK}$ and IRDye ${ }^{\circledast} 800 \mathrm{NOS}$ with respect to near-infrared fluorescence imaging of the cystic duct and cystic artery. At this moment, these dyes are not yet FDA approved, which also still is the case for IRDye ${ }^{\circledR} 800 \mathrm{CW}$, and are therefore not applicable for human clinical practice yet. The new dyes seem to be good alternatives for IRDye ${ }^{\circledR} 800 \mathrm{CW}$ 
in the visualization of the cystic duct and cystic artery in pigs. In order to be an alternative for the only dye that is at present available for human use, ICG, further studies and FDA approval are needed. 


\section{REFERENCES}

1. Vollmer CM Jr, Callery MP (2007) Biliary injury following laparoscopic cholecystectomy: why still a problem? Gastroenterology 133(3):1039-1041

2. Huang Q, Yao HH, Shao F, Wang C, Hu YG, Hu S et al (2014) Analysis of risk factors for postoperative complication of repair of bile duct injury after laparoscopic cholecystectomy. Dig Dis Sci 59(12):3085-3091

3. Viste A, Horn A, Ovrebo K, Christensen B, Angelsen JH, Hoem D (2015) Bile duct injuries following laparoscopic cholecystectomy. Scand J Surg 104(4):233-237

4. Way LW, Stewart L, Gantert W, Liu K, Lee CM, Whang K et al (2003) Causes and prevention of laparoscopic bile duct injuries: analysis of 252 cases from a human factors and cognitive psychology perspective. Ann Surg 237(4):460-469

5. Schols RM, Connell NJ, Stassen LP (2015) Near-infrared fluorescence imaging for real-time intraoperative anatomical guidance in minimally invasive surgery: a systematic review of the literature. World J Surg 39(5):1069-1079

6. Handgraaf HJ, Verbeek FP, Tummers QR, Boogerd LS, van de Velde CJ, Vahrmeijer AL et al (2014) Real-time near-infrared fluorescence guided surgery in gynecologic oncology: a review of the current state of the art. Gynecol Oncol 135(3):606-613

7. Autorino R, Zargar H, White WM, Novara G, Annino F, Perdona S et al (2014) Current applications of near-infrared fluorescence imaging in robotic urologic surgery: a systematic review and critical analysis of the literature. Urology 84(4):751-759

8. Schols RM, Bouvy ND, van Dam RM, Masclee AA, Dejong CH, Stassen LP (2013) Combined vascular and biliary fluorescence imaging in laparoscopic cholecystectomy. Surg Endosc 27(12):4511-4517

9. Verbeek FP, Schaafsma BE, Tummers QR, van der Vorst JR, van der Made WJ, Baeten Cl et al (2014) Optimization of nearinfrared fluorescence cholangiography for open and laparoscopic surgery. Surg Endosc 28(4):1076-1082

10. Schols RM, Lodewick TM, Bouvy ND, van Dam DA, Meijerink WJ, van Dam GM et al (2014) Near-infrared fluorescence laparoscopy of the cystic duct and artery in pigs: performance of a preclinical dye. J Laparoendosc Adv Surg Tech Part A 24(5):318-322

11. Tanaka E, Choi HS, Humblet V, Ohnishi S, Laurence RG, Frangioni JV (2008) Real-time intraoperative assessment of the extrahepatic bile ducts in rats and pigs using invisible nearinfrared fluorescent light. Surgery 144(1):39-48

12. Korb ML, Huh WK, Boone JD, Warram JM, Chung TK, de Boer E et al (2015) Laparoscopic fluorescent visualization of the ureter with intravenous IRDye800CW. J Minim Invasive Gynecol 22(5):799-806

13. Leung K (2004) IRDye 800CW-human serum albumin. Molecular imaging and contrast agent database (MICAD). BethesdA (MD).

14. Biosciences L-C. IRDye ${ }^{\circledast} 800 C W$ dye structures (2016). https://www.licor.com/bio/products/ reagents/irdye/800cw/structure.html.

15. Lange JF SL (2006) Best practice: De techniek van de laparoscopische cholecystectomie (Critical View of Safety [CVS]; Werkgroep Endoscopische Chirurgie van de Nederlandse Vereniging voor Heelkunde). http://www.nvgic.nl/richtlijnen/BestPracticeLaparoscopischeCholecystectomie. pdf.

16. Strasberg SM, Hertl M, Soper NJ (1995) An analysis of the problem of biliary injury during laparoscopic cholecystectomy. J Am Coll Surg 180(1):101-125

17. Penha FM, Rodrigues EB, Maia M, Meyer CH, Costa Ede P, Dib E et al (2013) Biochemical analysis and decomposition products of indocyanine green in relation to solvents, dye concentrations and laser exposure. Ophthalmologica 230(Suppl 2):59-67 
18. Kono Y, Ishizawa T, Tani K, Harada N, Kaneko J, Saiura A et al (2015) Techniques of fluorescence cholangiography during laparoscopic cholecystectomy for better delineation of the bile duct anatomy. Medicine (Baltimore) 94(25):e1005

19. Scheller MS, Unger RJ, Kelner MJ (1986) Effects of intravenously administered dyes on pulse oximetry readings. Anesthesiology 65(5):550-552

20. Ediriwickrema LS, Francis JH, Arslan-Carlon V, Dalecki PH, Brodie SE, Marr BP et al (2015) Intravenous injection of indocyanine green results in an artificial transient desaturation by pulse oximetry. Retin Cases Brief Rep 9(3):252-255

21. Baek HY, Lee HJ, Kim JM, Cho SY, Jeong S, Yoo KY (2015) Effects of intravenously administered indocyanine green on nearinfrared cerebral oximetry and pulse oximetry readings. Korean $J$ Anesthesiol 68(2):122-127 
CHAPTER 5

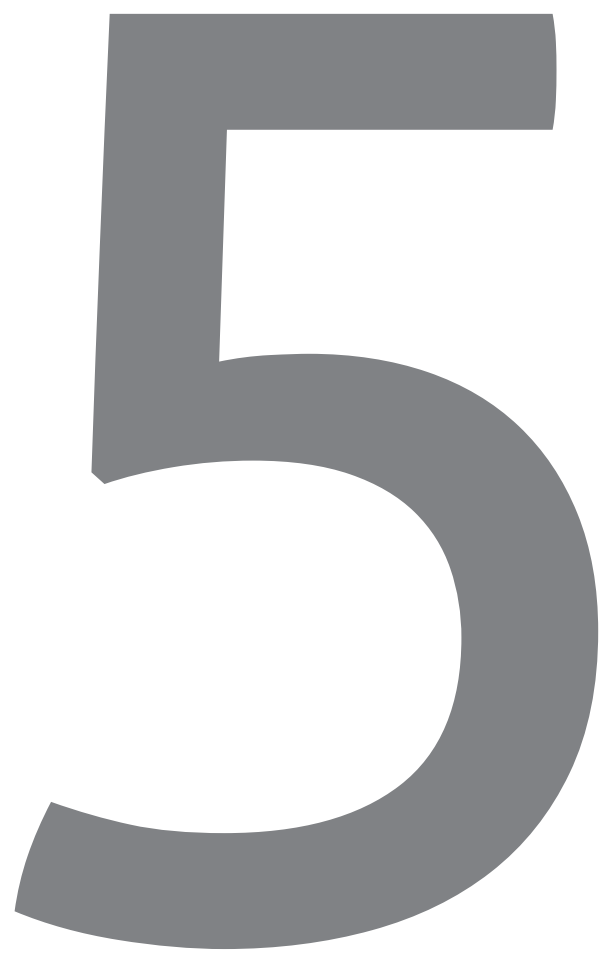




\section{Technical note: measuring the fluorescence intensity in NIRF cholangiography}

Jacqueline van den Bos, Rutger M. Schols, Sander M.J. van Kuijk, Fokko P. Wieringa, Nicole D. Bouvy, Laurents P.S. Stassen.

Journal of laparoendoscopic \& advanced surgical techniques 2019 [Epub ahead of print] 


\section{ABSTRACT}

\section{Aim}

To investigate whether different calculation methods to express fluorescence intensity (FI) as target-to background ratio are comparable and which method(s) match with human perception.

\section{Methods}

Comparison of three calculation methods from current literature (OsiriX, ImageJ and Photoshop) to objectify FI intensity during laparoscopic cholecystectomy measured at the exact same locations within recorded images of two categories: ex-vivo and in-vivo. Currently applied formulas to present $\mathrm{FI}$ in relation to the background (BG) signal are compared with the subjective assessment by the human observers. These three formulas are 'Signal contrast $=$ (FI in fluorescence regions - Fl in BG) / 255'; 'Target-to-background ratio $=(\mathrm{Fl}$ of target $-\mathrm{Fl}$ of BG) / FI of BG'; 'Signal-to-background ratio = FI of Cystic duct / FI of liver' and 'Target-to-background ratio = (FI of Target - Noise) / (FI of BG - Noise)'.

\section{Results}

In our evaluation OsiriX and ImageJ provided similar results, while OsiriX values were structurally slightly lower compared to ImageJ. Values obtained via Photoshop were less evidently related to those obtained with OsiriX and ImageJ. The formula Target-tobackground ratio $=$ (Fl of Target - Noise $) /($ Fl of BG - Noise) was less corresponding with human perception compared to the other used formulas.

\section{Conclusions}

Fluorescent intensity results based on measurements using the programs OsiriX and ImageJ are similar, allowing for comparison of results between these programs. Results using Photoshop differ significantly, making direct comparison impossible. This is an important finding when interpreting study results. We propose to report both target and background $\mathrm{Fl}$ in manuscripts, so that proper interpretation between articles can be made. 


\section{INTRODUCTION}

Intraoperative near infrared fluorescence (NIRF) imaging is gaining both more applications and more users. The current main applications for the use of NIRF imaging with indocyanine green (ICG) in gastrointestinal surgery are: visualization of the cystic duct during laparoscopic cholecystectomy, perfusion assessment of gastrointestinal anastomoses and lymph node identification (1-5). The growing popularity of NIRF applications is accompanied by an increasing number of (pre)clinical studies published on this topic. In these studies, different applications, different fluorescent dyes and different methods for reporting the intensity of the signal are used. For objective inter-comparison between studies, a uniform objective quantification of the signal is mandatory. For instance, in the application of NIRF angiography, the fluorescent signal is reported in several ways, like: 'adequate/sufficient or inadequate/ insufficient' (6), 'good, average or bad' (7). It is difficult to compare the outcomes of studies when only such subjective evaluation is used. Our research group and many others use objective assessment of the fluorescence intensity (FI) (8). A review we recently performed showed that both different software packages and different formulas are used, still making inter-comparison of studies difficult (9). In random order, these software programs are OsiriX, ImageJ and Photoshop. Commonly used formulas in current literature are presented in Table 1.

The aim of this technical note is to investigate whether measurements concerning FI reported in current literature are comparable and matching with human visual perception. A second aim is to evaluate the formulas that are currently used to correct the signal of the target with respect to the signal of the background, and to provide an advice on which formula to use in future research.

Table 1 Applied formulas as reported in literature and their results

\begin{tabular}{|c|c|c|c|}
\hline Reported formulas in literature & $\begin{array}{l}\text { Type of image in } \\
\text { articles }\end{array}$ & $\begin{array}{l}\text { Mean accuracy } \\
\text { when using grey- } \\
\text { value }\end{array}$ & $\begin{array}{l}\text { Mean accuracy } \\
\text { when using blue- } \\
\text { value }\end{array}$ \\
\hline $\begin{array}{l}\text { Signal contrast }= \\
\text { (FI in fluorescence regions }-\mathrm{Fl} \text { in } \\
\mathrm{BG}) / 255\end{array}$ & Black and white & 10.7 / 18 (range 9-13) & 11 / 18 (range 9-14) \\
\hline $\begin{array}{l}\text { Target-to-background ratio }= \\
(\mathrm{FI} \text { of target }-\mathrm{FI} \text { of } \mathrm{BG}) / \mathrm{FI} \text { of } \mathrm{BG}\end{array}$ & $\begin{array}{l}\text { Color (fluorescent } \\
\text { image in blue) }\end{array}$ & 10.3 / 18 (range 9-11) & 8.8 / 18 (range 6-12) \\
\hline $\begin{array}{l}\text { Signal-to-background ratio = } \\
\text { FI of Cystic duct / FI of liver }\end{array}$ & $\begin{array}{l}\text { Both black and } \\
\text { white and color } \\
\text { (fluorescent image } \\
\text { in blue or green) }\end{array}$ & 10.2 /18 (range 9-11) & 9.1 / 18 (range 7-12) \\
\hline $\begin{array}{l}\text { Target-to-background ratio = } \\
\text { (Fl of Target - Noise) / (FI of BG - } \\
\text { Noise) }\end{array}$ & Black and white & 8.5 / 18 (range 7-10) & 7.3 / 18 (range 5-10) \\
\hline
\end{tabular}

Fl: Fluorescence intensity; BG: Background 


\section{METHODS}

To explore software packages and formulas used to report Fl in laparoscopic cholecystectomy, the results of an earlier performed systematic literature search were used (9). 28 articles were screened for the use of software packages to measure FI and the applied formulas to quantify the intensity of the signal in the cystic duct compared to the background fluorescence. The identified software packages were then used in a controlled ex vivo setting to measure the FI of ICG in known concentrations with the laparoscope at a fixed distance. Subsequently, FI was measured using these programs on screenshots made during NIRF laparoscopic cholecystectomies. Finally, with the aforementioned screenshots, the formulas presented in literature were evaluated by assessing which formula corresponded best with the subjective assessment by the observers. See Figure 1 for an overview of study methods.

\section{Analysis of NIRF images obtained during ex vivo experiments}

Images from an earlier ex vivo experiment were used (9). In this ex vivo experiment, ICG was diluted with $35 \mathrm{mg} / \mathrm{ml}$ albumin in a $0.9 \% \mathrm{NaCl}$ solution to known concentrations. For the current experiment, images from dilutions of $5 \mathrm{mg} / \mathrm{ml} \mathrm{ICG,} 0.5 \mathrm{mg} / \mathrm{ml}$ and $0.001 \mathrm{mg} / \mathrm{ml}$ were used. For each dilution, 9 times $1 \mathrm{ml}$ of the dilution was placed on a wells plate. Images were obtained by making screenshots (PNG image) in the videos (mp4) that were made with the laparoscopic system (D-Light-P Fluorescence system, Karl Storz, Tuttlingen, Germany). For all concentrations, the FI was measured at $5 \mathrm{~cm}$ and 9, 10, 11, 12, 13 and $14 \mathrm{~cm}$ distance. The Fl was measured at three points in the image, by two observers. The points of interest were placed on the exact same location; the exact same pixels were included, in the middle of the wells-cup, while no light reflections were included in the regions of interest.

\section{Analysis of NIRF images in laparoscopic cholecystectomy}

We randomly selected four laparoscopic cholecystectomies with NIRF imaging; these concern elective procedures performed in the Maastricht University Medical Center (Maastricht, the Netherlands). Screenshots were collected from the surgical videos. In these screenshots, at least the liver and cystic duct had to be visible in fluorescence light. In all screenshots, the Fl was measured in three regions of interest (ROIs) in the cystic duct, three ROIs in the liver (two on the right of the cystic duct, one left). The regions in the liver were used as background reference. Three other regions in the surroundings were measured and were assessed as noise. The Fl in these ROls was measured in all programs by two observers and both the grey-value and blue-value were measured. Again, care was taken that per image, the ROI positions were pixel to pixel aligned on the same locations in all software programs. See Figure 2 for an example on the chosen regions of interest in a screenshot.

Determination of a conversion formula for comparison of data from different programs measured $\mathrm{Fl}$ in the respective programs was compared in order to obtain a conversion 


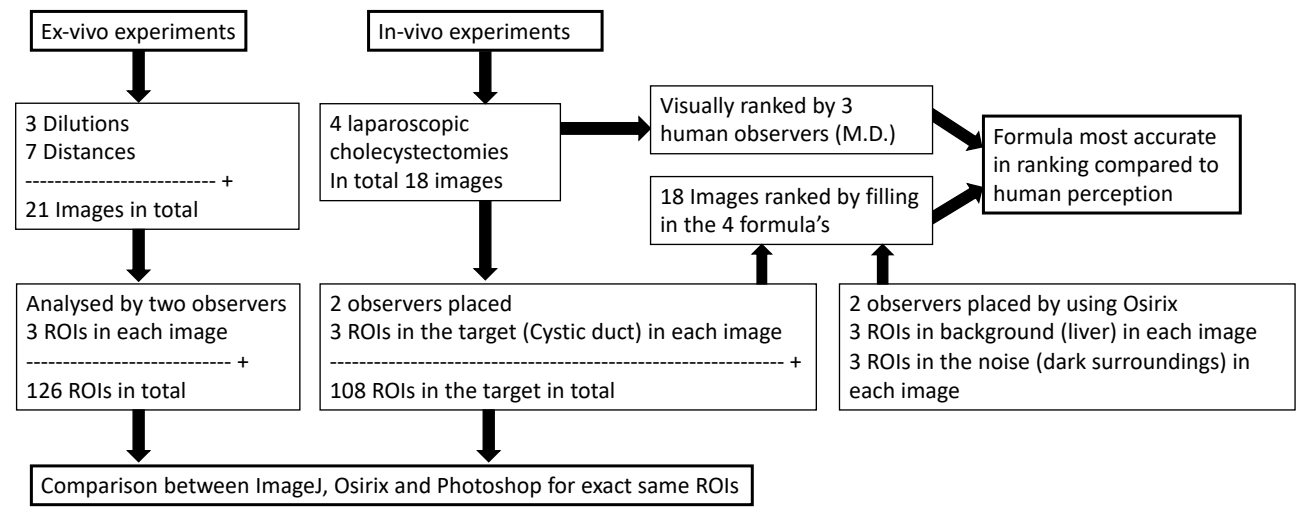

$\mathrm{ROI}=$ Region of interest M.D.= Medical doctor.

Figure 1 Overview of Study methods

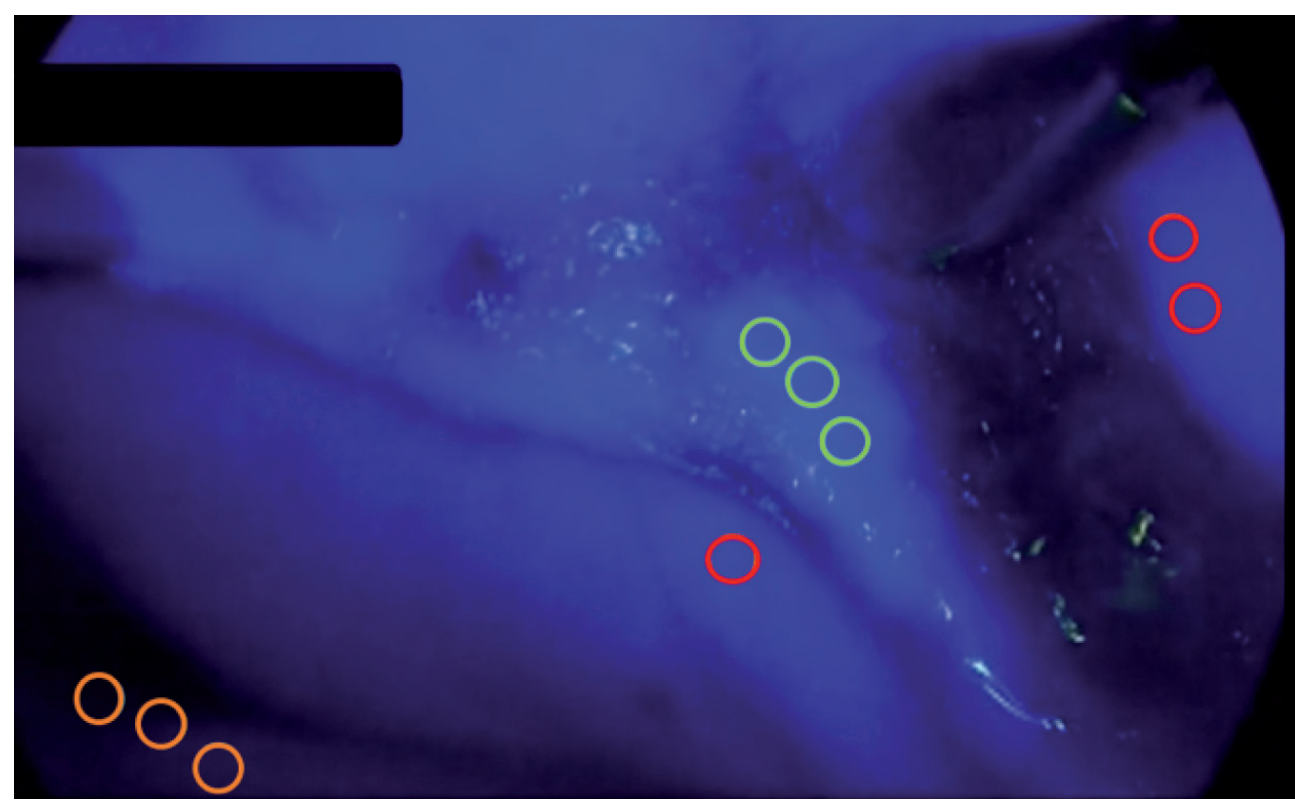

The ROIs are color-coded as follows: cystic duct in green, liver (Background) in red and noise in orange.

Figure 2 Example of assigned Regions of Interest (ROI) for in-vivo fluorescence intensity measurement

formula to enable comparison of obtained FI in the one study with another. Analysis was performed in SPSS version 23 (IBM corp. Armonk, NY). First, observations were visualized in a scatter plot. A linear regression analysis was performed to assess how results of one formula could be converted to another. These analyses were initially performed separately for the ex vivo and the in vivo measurements. Then, agreement was quantified using an intraclass correlation coefficient and linear regression analysis. 


\section{Comparison of the objectively measured and calculated fluorescence intensity with subjective assessment}

The assessed screenshots from the aforementioned NIRF laparoscopic procedures were ordered from "very clearly visible fluorescent delineation" of the cystic duct compared to the surroundings to "weak fluorescence" of the cystic duct compared to the surroundings based on the opinion of three experts. This subjective assessment was compared with the results from filling in the formulas identified from literature for both the measured grey values and the measured blue-values. The FI and BG intensity used therefore were measured with Osirix. A difference in ranking of more than 5 places was considered a mismatch. A ranking score within 5 places was scored as an accurate match between subjective order and calculated order with the respective formula. The used ROIs were the same for all four formulas as the example in Figure 2. The ROls from both observers were filled in in all formulas.

\section{RESULTS}

\section{Programs used to objectively measure fluorescence intensity}

In the literature, three software programs were identified for objective quantification of the signal, namely OsiriX (Pixmeo, Bernex, Switzerland) (2, 8, 10-12), ImageJ (National Institutes of Health, Bethesda, USA) (13-15) and Photoshop CS (Adobe systems, San Jose, USA) (16). OsiriX, ImageJ and Photoshop were used in respectively five, three and one of the reviewed studies.

\section{Ex vivo NIRF-images analysis}

Twenty-one images were analyzed for the three ICG concentrations $(5 \mathrm{mg} / \mathrm{ml}, 0.5 \mathrm{mg} / \mathrm{ml}$ and $0.001 \mathrm{mg} / \mathrm{ml}$ ) at different distances. Because two observers analyzed three points in every image, a total of 126 points were analyzed. As shown in Figure 3, the values obtained with ImageJ are very strongly related to the FI measured with OsiriX (Intraclass correlation coefficient $=1.0 ; R^{2}=0.9999 ; p<0.001$ ). With the regression analysis, a formula was obtained to convert the measured value from ImageJ to a value when OsiriX would have been used:

Value measured with OsiriX $=-0.44+0.98$ (Value measured with Image J).

In reverse, the formula value for conversion from OsiriX to ImageJ would be:

Value measured with ImageJ $=0.46+1.02$ (value measured with OsiriX).

When comparing the values obtained with OsiriX with the values obtained with Photoshop, also a statistical significant relation between results was found, however with a lower interclass correlation coefficient (Interclass correlation coefficient $=0.78 ; R^{2}=0.93 ; p<0.001$ ). As shown in Figure 4, no obvious straight line is formed by the data points obtained with Photoshop and OsiriX (in contrast to comparing between OsiriX and ImageJ). Therefore, no 


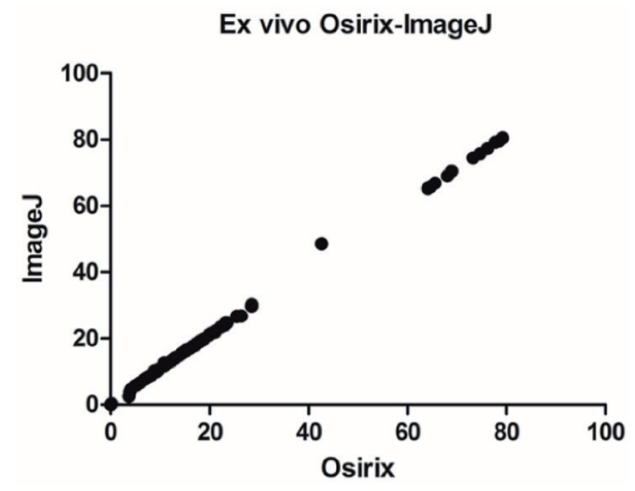

Figure 3 Relation between measured fluorescence intensity using ImageJ and OsiriX in ex vivo images

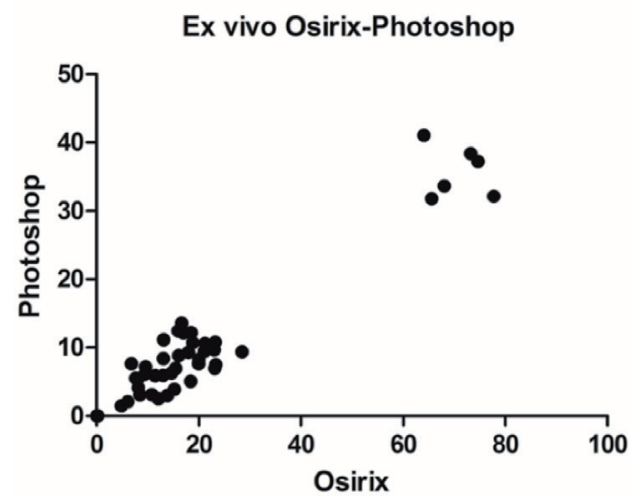

Figure 4 Relation between measured fluorescence intensity using Photoshop and OsiriX in ex vivo images

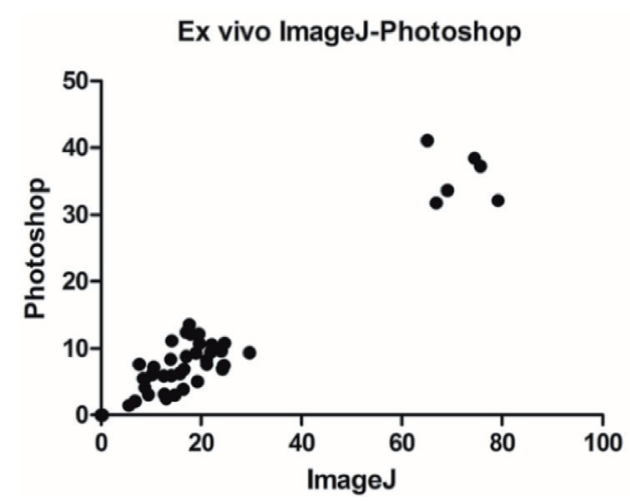

Figure 5 Relation between measured fluorescence intensity using Photoshop and ImageJ in ex vivo images

straightforward formula to convert between values derived from OsiriX versus Photoshop could be obtained using a regression analysis. An approximate conversion formula based on a linear regression analysis would be:

$$
\text { OsiriX }=1.26+1.88 \text { (value measured with Photoshop) }
$$

The inverse approximate conversion would be:

$$
\text { Photoshop value }=0.01+0.49(\text { value measured with OsiriX })
$$

Note that values obtained with OsiriX are on average roughly two times as high as values measured with Photoshop (while using the same FI as input).

Comparing measured $\mathrm{FI}$ values between Photoshop and ImageJ, a similar statistical significant relation is found (Interclass correlation $=0.78 ; R^{2}=0.93 ; p<0.001$ ). This relation is shown in Figure 5: 
Value obtained with Image J $=1.88+1.90$ (value obtained with Photoshop).

With the corresponding inverse approximate conversion:

Value obtained with Photoshop $=-0.26+0.49 \times$ (Value obtained with ImageJ).

In vivo NIRF-images analysis (from laparoscopic cholecystectomy)

Eighteen screenshots in four patients were analyzed. These patients all underwent elective laparoscopic cholecystectomy for symptomatic cholecystitis. In these images FI was measured on exact the same ROls with the three software programs, with two sets of ROIs chosen independently by two observers. Comparing OsiriX and ImageJ based on the measured gray-values in the images, revealed a very strong correlation (Intercorrelation coefficient $=1 ; R^{2}=1 ; p<0.001$ ) and when data is plotted, a straight line can be drawn between the points, as is shown in Figure 6. The conversion formulas from linear regression analysis between OsiriX and ImageJ are:

OsiriX $=-0.69+1.00$ (value obtained with ImageJ), and Image $=0.75+1.00$ (value obtained with OsiriX)

When measuring the blue-values, a perfect correlation (interclass correlation $=1 ; R^{2}=1.00 ; p$ $<0.001)$ was found with the following conversion formulas:

Blue-value OsiriX $=0.42+1.00$ (blue-value obtained with Image J), and

Blue value Image $\mathrm{J}=-0.30+1.00$ (blue-value obtained with OsiriX)

In the in vivo experiments, statistically there seems to be a relation between the values measured in Photoshop and OsiriX (interclass correlation $=0.76 ; R^{2}=0.63 ; p<0.001$ ). However, as shown in figure 7, no straightforward conversion formula can be identified to compare between studies.

This is the same for Photoshop and ImageJ (Interclass correlation $=0.76 ; R^{2}=0.63 ; p$ $<0.0001$ ), see also figure 8 .

For the blue-values outcomes are comparable. Between OsiriX and Photoshop an interclass correlation of 0.96 was found $\left(R^{2}=0.92, P<0.001\right)$, between ImageJ and Photoshop the interclass correlation was $0.96\left(R^{2}=0.93, P<0.001\right)$.

\section{Reported formulas for assessment of fluorescence intensity:}

As mentioned, the $\mathrm{Fl}$ in the articles is often presented using a formula. The four formulas identified are reported in Table 1.

Using the described method, the formula Signal contrast $=(\mathrm{Fl}$ in fluorescence regions $-\mathrm{F})$ in BG) / 255 seemed to be the most comprehensive with what the experts reported as their visual perceptions. However, as shown in table 1, differences are small.

As the formulas were applied to grey values in colour images with fluorescence in blue, we also tested these formulas using the blue-value. From this refinement, also the formula Signal contrast $=(F /$ in fluorescence regions $-F I$ in BG) / 255 emerged as showing the 

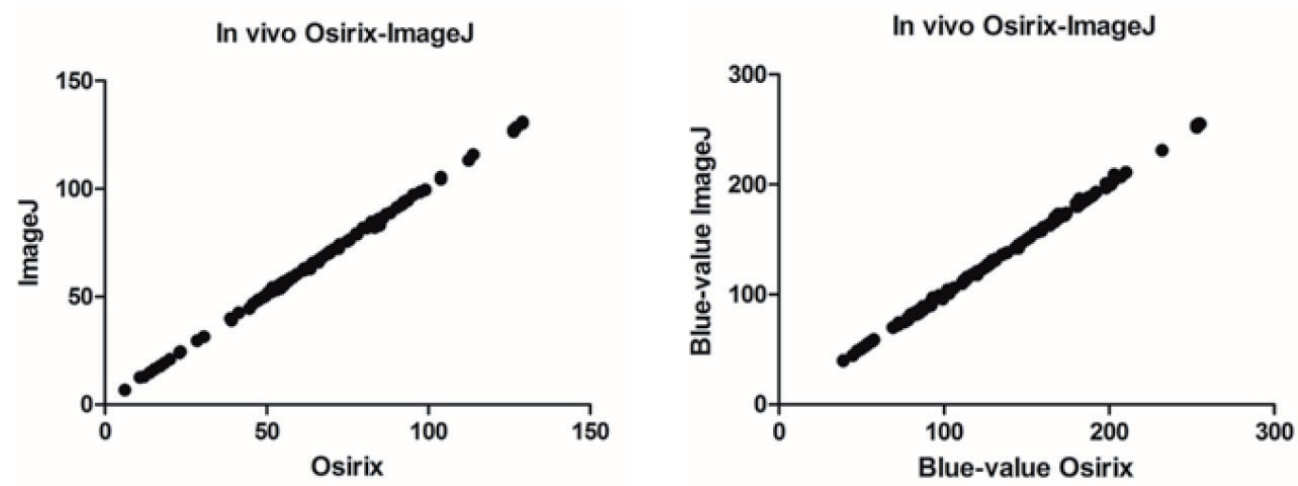

Figure 6 Relation between measured fluorescence intensity using Image J and OsiriX in in-vivo images for both grey-value and blue-value
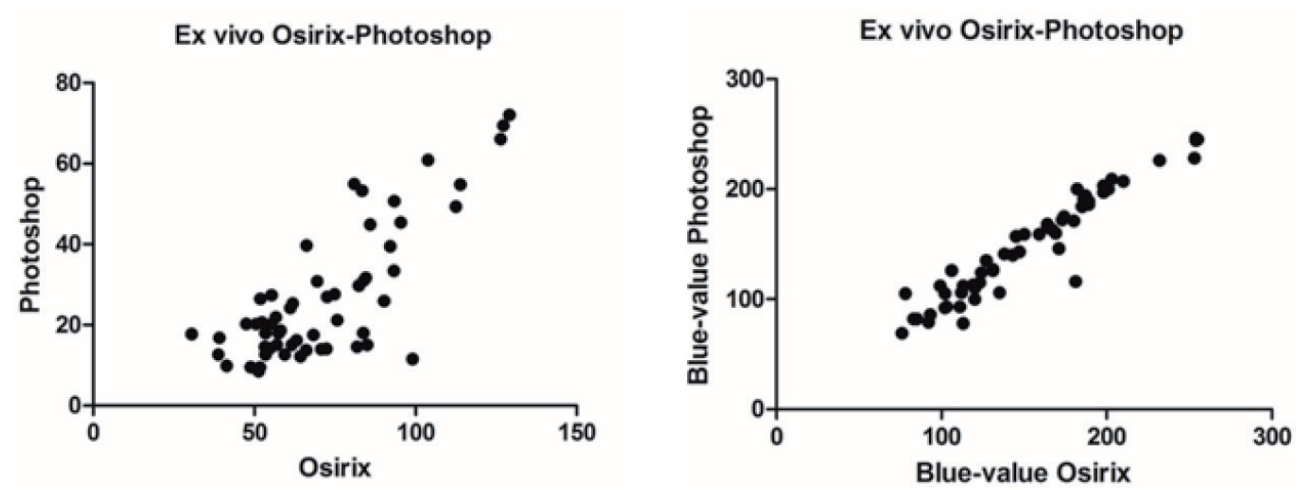

Figure 7 Relation between measured fluorescence intensity using Photoshop and OsiriX in in-vivo images

In vivo ImageJ-Photoshop

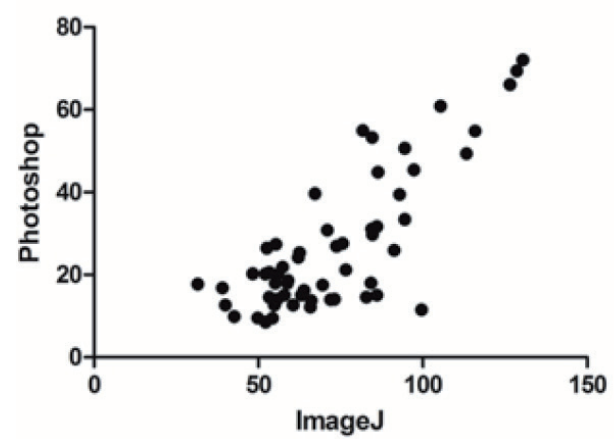

In vivo ImageJ-Photoshop

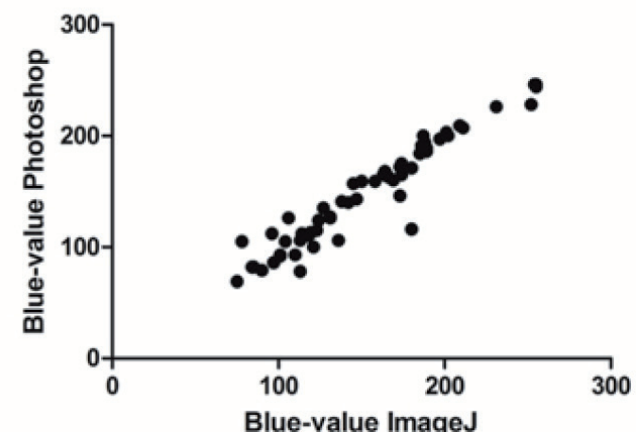

Figure 8 Relation between measured fluorescence intensity using Photoshop and ImageJ

strongest match with the subjective order of Fl of the ductus. However, the formula Signalto-background ratio = Fl of Cystic duct / Fl of liver was somewhat more accurate than Target-tobackground ratio $=($ Fl of target - Fl of BG $) / F I$ of BG. 


\section{DISCUSSION}

Despite an increasing number of publications in the field of NIRF imaging, no standard yet exists for objective assessment of fluorescence images. This problem has recently been highlighted by reports from the American Association of Physicists in Medicine (AAPM) (18). Different software packages are used to quantify the fluorescent signal, and also different formulas are applied to correct for the background fluorescence. The latter is important as for human visual perception not only the absolute signal of the target is important, but also the contrast in relation to its surroundings. Such a formula actually is an imitation of the human visual perception system that naturally uses such contrast for object identification. In the present study we focused on two goals. First, to investigate whether different software packages used for measuring FI reported in the current literature yield comparable results. Secondly to identify the formulas used in literature to calculate a target-to background ratio and try to provide an advice on what formula to use in future research for comparison of studies.

In our ex-vivo analysis, a very clear relation between values measured with OsiriX and ImageJ was detected. The values obtained with OsiriX were approximately 0.5 units lower than those obtained with ImageJ. The relation between the values from Photoshop and from the other two programs was less evident as illustrated by a lower correlation coefficient.

From our in vivo images, a slightly different conversion factor between OsiriX and ImageJ was observed. This may be due to the fact that images from an operative field are less standardized and influenced by subtle differences in scattering and light intensity. A possible explanation for the differences in correlation between the results from the different programs is a difference in measuring which is probably more likely to occur when using Photoshop. It is our experience that it seems more difficult to exactly select the same pixels for analysing the ROI. The various applied analysis programs were treated as a black box within this study. However, the software that provides results that are matching well with human perception seems to be more promising than others.

Apart from differences in used software, different formulas are used to present the Fl. It is essential to take the signal from the surroundings due to uptake of the dye into account, because this influences the contrast as observed by the surgeon (9). When using a formula, the influence of the background is taken into account and thereby might provide a number more consistent with human perception. Several formulas are used that use target- and background signal, but in different dependency. It is difficult to decide on theoretical grounds which approach is best. Some points of attention should be kept in mind. Of course, the ROI's should be chosen carefully, reflecting the signal of the subject best. Next, when using a formula, the distance between the laparoscope and the background should 
be about the same as the distance between the target and the laparoscope. An observation, for which the present way of measuring doesn't correct, is the fact that a small darker zone is observed surrounding the cystic duct, which influences the observed contrast, but is not taken into account when taking the liver as background.

In current literature, four different formulas were found. We tested these formulas against the assessment by the human eye. One formula seemed to be most accurate. This was the formula used by Kono et al (16), namely Signal contrast = (Fl in fluorescence regions - Fl in BG) /255. However, even though this was the best of the four formulas, there was not a $100 \%$ match between the subjective order and obtained order with this formula and differences with the other formulas were small. The only deviating formula was the formula 'Targetto-background ratio $=(\mathrm{Fl}$ of Target - Noise) $/$ (FI of BG - Noise)' which provided results less compatible with human perception. Most likely this is due to the noise which is taken account in this formula. The cystic duct is often directly surrounded by a dark signal due to the shape of the cystic duct and shows more light reflections compared to the liver. Therefore, this cystic duct is sometimes easier recognizable with the naked eye than what is suggested by the outcomes of the formulas. Based on these observations it is not possible to advice one formula or the other. From our own experience, it is of great importance, independent from the formula used, that the ROI for signal analysis, are very carefully chosen. In the digital image, as discussed earlier, subtle differences in signal intensity and scattering occur within the same ROI, for example the cystic duct.

Both OsiriX and ImageJ measure the intensity of the signal on a grey scale. However, the fluorescence signal is most often presented in color images, especially in blue or green. The equipment used in the present study gives a blue image for fluorescence. This is the reason why not only the grey values but also the blue values were measured. However, we observed comparable outcomes using both methods. These results may be influenced by the fact that the used fluorescence system gives a very dark background, hardly without any observable color. The background reference values and therefore the results of the formula might have been different when had been used that contains a more mixed light source, filtering out less of the white light and therefor showing more of the environment.

A limitation of this study is that it no uniformity existed in the equipment used between studies nor in the exact application of the technique. There are more relevant factors of influence than only the software used $(16,19)$. The equipment itself, the used laparoscope and the software in the laparoscopic system is of influence. Also, the timing of the application of the dye yields different results. As is shown in earlier studies, a longer time between ICG administration and assessment causes a more fluorescent cystic duct compared to the liver than an injection just 30 minutes before assessment $(13,14)$. And last, probably the most important factor is the selection of the ROI's and the background, which is a subjective 
action. We advocate minimizing this influence by choosing three points in both target and background and use the mean value of these three. However, the risk for selection bias remains.

\section{CONCLUSION}

When comparing fluorescence values obtained from analysis with different programs, this is easiest with those from OsiriX and ImageJ. A conversion with a correction factor as indicated in this article is possible, enabling the reader to interpret the presented values correctly. The relation between these two programs and Photoshop is less clear, hindering comparison of results. Furthermore, to overcome the present use of different formulas by different authors to further evaluate the signal, we propose to report both $\mathrm{ROI}$ and Background $\mathrm{FI}$ in the manuscript. This enables calculations to be made between articles independently of the preferred formula by the authors. Lastly in interpreting results, other influences on the $\mathrm{Fl}$ and the risk for selection bias in chosen regions of interest for evaluation of the signal should be taken into account. 


\section{REFERENCES}

1. Osayi SN, Wendling MR, Drosdeck JM, Chaudhry UI, Perry KA, Noria SF, et al. Near-infrared fluorescent cholangiography facilitates identification of biliary anatomy during laparoscopic cholecystectomy. Surgical endoscopy. 2015;29(2):368-75.

2. Schols RM, Bouvy ND, van Dam RM, Masclee AA, Dejong CH, Stassen LP. Combined vascular and biliary fluorescence imaging in laparoscopic cholecystectomy. Surg Endosc. 2013;27(12):4511-7.

3. Ishizawa T, Bandai Y, Hasegawa K, Kokudo N. Fluorescent cholangiography during laparoscopic cholecystectomy: indocyanine green or new fluorescent agents? World J Surg. 2010;34(10):2505-6.

4. van den Bos J, Al-Taher M, Schols RM, van Kuijk S, Bouvy ND, Stassen LPS. Near-Infrared Fluorescence Imaging for Real-Time Intraoperative Guidance in Anastomotic Colorectal Surgery: A Systematic Review of Literature. J Laparoendosc Adv Surg Tech A. 2018;28(2):157-67.

5. Zeng HC, Hu JL, Bai JW, Zhang GJ. Detection of Sentinel Lymph Nodes with Near-Infrared Imaging in Malignancies. Mol Imaging Biol. 2018.

6. Boni L, David G, Dionigi G, Rausei S, Cassinotti E, Fingerhut A. Indocyanine green-enhanced fluorescence to assess bowel perfusion during laparoscopic colorectal resection. Surgical endoscopy. 2016;30(7):2736-42.

7. Ris F, Hompes R, Cunningham C, Lindsey I, Guy R, Jones O, et al. Near-infrared (NIR) perfusion angiography in minimally invasive colorectal surgery. Surgical endoscopy. 2014;28(7):2221-6.

8. Schols RM, Bouvy ND, Masclee AA, van Dam RM, Dejong CH, Stassen LP. Fluorescence cholangiography during laparoscopic cholecystectomy: a feasibility study on early biliary tract delineation. Surg Endosc. 2013;27(5):1530-6.

9. van den Bos J, Wieringa FP, Bouvy ND, Stassen LPS. Optimizing the image of fluorescence cholangiography using ICG: a systematic review and ex vivo experiments. Surgical endoscopy. 2018.

10. van den Bos J, Al-Taher M, Hsien SG, Bouvy ND, Stassen LP. Near-infrared fluorescence laparoscopy of the cystic duct and cystic artery: first experience with two new preclinical dyes in a pig model. Surgical endoscopy. 2017.

11. Schols RM, Lodewick TM, Bouvy ND, van Dam DA, Meijerink WJ, van Dam GM, et al. Nearinfrared fluorescence laparoscopy of the cystic duct and artery in pigs: performance of a preclinical dye. J Laparoendosc Adv Surg Tech A. 2014;24(5):318-22.

12. Figueiredo JL, Siegel C, Nahrendorf M, Weissleder R. Intraoperative near-infrared fluorescent cholangiography (NIRFC) in mouse models of bile duct injury. World J Surg. 2010;34(2):336-43.

13. Tsutsui N, Yoshida M, Nakagawa H, Ito E, Iwase R, Suzuki N, et al. Optimal timing of preoperative indocyanine green administration for fluorescent cholangiography during laparoscopic cholecystectomy using the PINPOINT(R) Endoscopic Fluorescence Imaging System. Asian J Endosc Surg. 2017.

14. Boogerd LSF, Handgraaf HJM, Huurman VAL, Lam HD, Mieog JSD, van der Made WJ, et al. The Best Approach for Laparoscopic Fluorescence Cholangiography: Overview of the Literature and Optimization of Dose and Dosing Time. Surg Innov. 2017;24(4):386-96.

15. Gao Y, Li M, Song ZF, Cui L, Wang BR, Lou XD, et al. Mechanism of dynamic near-infrared fluorescence cholangiography of extrahepatic bile ducts and applications in detecting bile duct injuries using indocyanine green in animal models. J Huazhong Univ Sci Technolog Med Sci. 2017;37(1):44-50.

16. Kono Y, Ishizawa T, Tani K, Harada N, Kaneko J, Saiura A, et al. Techniques of Fluorescence Cholangiography During Laparoscopic Cholecystectomy for Better Delineation of the Bile Duct Anatomy. Medicine (Baltimore). 2015;94(25):e1005.

17. Verbeek FP, Schaafsma BE, Tummers QR, van der Vorst JR, van der Made WJ, Baeten $\mathrm{Cl}$, et al. Optimization of near-infrared fluorescence cholangiography for open and laparoscopic surgery. Surg Endosc. 2014;28(4):1076-82. 
18. Pogue BW, Zhu TC, Ntziachristos V, Paulsen KD, Wilson BC, Pfefer J, et al. Fluorescenceguided surgery and intervention - An AAPM emerging technology blue paper. Med Phys. 2018;45(6):2681-8.

19. Hoogstins C, Burggraaf JJ, Koller M, Handgraaf H, Boogerd L, van Dam G, et al. Setting Standards for Reporting and Quantification in Fluorescence-Guided Surgery. Mol Imaging Biol. 2018. 
PART II 
NEAR INFRARED FLUORESCENCE IMAGING IN COLORECTAL SURGERY 


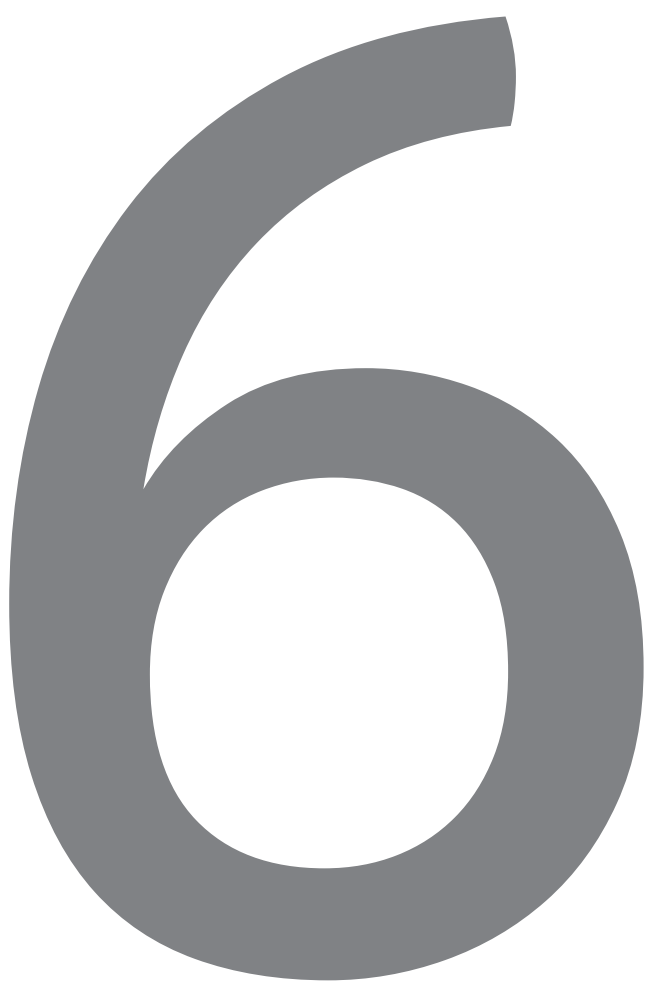




\section{Near-Infrared fluorescence imaging for real-time intraoperative guidance in anastomotic colorectal surgery: a systematic review of literature}

Jacqueline van den Bos, Mahdi Al-Taher, Rutger M. Schols, Sander van Kuijk, Nicole D. Bouvy, Laurents P.S. Stassen.

Journal of laparoendoscopic \& advanced surgical techniques 2017 28(2):157-167 


\section{ABSTRACT}

\section{Purpose}

The aims of this review are to determine the feasibility of near-infrared fluorescence (NIRF) angiography in anastomotic colorectal surgery and to determine the effectiveness of the technique in improving imaging and quantification of vascularization, thereby aiding in decision making as to where to establish the anastomosis.

\section{Methods}

A systematic literature search of PubMed and EMBASE was conducted. Searching through the reference lists of selected articles identified additional studies. All English language articles presenting original patient data regarding intraoperative NIRF angiography were included without restriction of type of study, except for case reports, technical notes, and video vignettes. The intervention consisted of intraoperative NIRF angiography during anastomotic colorectal surgery to assess perfusion of the colon, sigmoid, and/or rectum. Primary outcome parameters included ease of use, added surgical time, complications related to the technique, and costs. Other relevant outcomes were whether this technique changed intraoperative decision making, whether effort was taken by the authors to quantify the signal and the incidence of postoperative complications.

\section{Results}

Ten studies were included. Eight of these studies make a statement about the ease of use. In none of the studies complications due to the use of the technique occurred. The technique changed the resection margin in $10.8 \%$ of all NIRF cases. The anastomotic leak rate was $3.5 \%$ in the NIRF group and $7.4 \%$ in the group with conventional imaging. Two of the included studies used an objective quantification of the fluorescence signal and perfusion, using ROIs (Hamamatsu Photonics) and IC-Calc respectively.

\section{Conclusions}

Although the feasibility of the technique seems to be agreed on by all current research, large clinical trials are mandatory to further evaluate the added value of the technique. 


\section{INTRODUCTION}

Anastomotic leakage is the most feared complication in colorectal surgery. With an incidence of $3 \%-15 \%{ }^{1-8}$ in colorectal surgery, it remains a common problem. Anastomotic leakage concerns a severe complication that leads to significant morbidity, prolonged hospital stay, considerable extra costs, and increased short-term and long-term mortality. ${ }^{6-9}$ Known risk factors for anastomotic leakage are male sex, American Society of Anesthesiologists-score above 3, chronic steroid use, preoperative weight loss, smoking, preoperative chemotherapy, disease location (infra-peritoneal anastomosis), and prolonged operation time. ${ }^{6-8,10}$ Karliczek et al. ${ }^{11}$ discussed in their article that there is a lack of a reliable intraoperative predictive test for anastomotic leakage by the operating surgeon. Intraoperatively, the selection of an optimal site for anastomosis is now depending on subjective clinical indicators such as color of the bowel wall, palpable or visible pulsations of the mesenteric arteries, or bleeding of the resection margins. ${ }^{12}$ Tests to determine the colorectal anastomotic integrity are air leak testing or more invasive or complex techniques such as endoscopic visualization, Dopplerflowmetry, $\mathrm{pH}$ readings in the colon, all of which are not very convincing techniques according to the review by Nachiappan et al. ${ }^{12}$ Also, these tests are performed after construction of the anastomosis, while a predictor before making the anastomosis could prevent anastomotic leakage.

It is thought that a better vascularization of the anastomosis will cause less anastomotic leaks. ${ }^{13-16}$ There is a large variability of the colonic vascular anatomy and many patients suffer from arteriosclerotic disease of the mesenteric vasculature. ${ }^{17}$ Therefore, an easy and reliable method to assess the vascularization is desirable. With near-infrared fluorescence (NIRF) imaging it is expected that real-time visualization of the vasculature can be improved and, ultimately, anastomotic leaks reduced. The use of fluorescence angiography was first described in 1976 for choroid angiography ${ }^{18}$ and is common practice in ophthalmology. Applying the technique intraoperatively during colorectal surgery is comparable to this first application; by intravenous administration of the dye and its intravascular transportation, the vessels in the target region can be seen by illumination of the fluorescent dye.

Indocyanine green (ICG) is the most frequently used NIRF dye in humans. ICG is a sterile tricarbocyanine dye composed of N-hydro-3,3,3',3-tetramethyl-1,1-di(4-sulfobutyl)4,5,4'4'5-dibenzoindotricarbocyanine hydroxide sodium and absorbs light between 790 and $805 \mathrm{~nm}$ and re-emits light with an excitation wavelength of $835 \mathrm{~nm} .{ }^{19,20}$ Since a better vascularization of the bowel anastomosis is thought to result in less anastomotic leakage, identifying the degree of vascularization through fluorescence angiography might help reduce the risk of anastomotic leakage.

The first objective of the review was to determine the feasibility of NIRF angiography in anastomotic colorectal surgery, with emphasis on ease of use, added surgical time and complications related to the technique. A second objective was to determine the 
effectiveness of the technique in improving imaging of vascularization, thereby aiding in the decision making of where to make the anastomosis. Attention was given whether the signal was quantified or validated in an objective manner or only subjectively assessed.

\section{MATERIALS AND METHODS}

This study was registered in PROSPERO under the number: CRD42015025514 (www.crd.york. ac.uk/PROSPERO/display_record.asp?ID=CRD42015025514).

\section{Search Strategy}

A systematic literature search was performed in PubMed and EMBASE. The following search strategy was followed: ("Indocyanine Green" [Mesh] OR Indocyanine Green OR Infracyanine Green OR ICG OR "Fluorescent Dyes" [Mesh] OR Fluorescent Dyes OR Near-Infrared Fluorescence ORFluorescent imaging OR Fluorescence imaging OR Near Infrared Imaging) AND ("Laparoscopy" [Mesh] OR Laparoscopy OR laparoscopic OR Robotic surgery) AND ("Anastomosis, Surgical" [Mesh] OR Bowel anastomosis OR Anastomotic bowel surgery OR "Colorectal Surgery" [Mesh] OR Colorectal Surgery).

First, titles and abstracts were screened. In case of uncertainty, full text reports were read to assess eligibility. To complete the search, references within selected articles were searched as well. The search was performed by 2 independent reviewers (JvdB and MAT) who selected potentially relevant articles by title and abstract.

\section{Inclusion and exclusion criteria}

Articles had to be written in English. The aim of the literature search was to identify articles that reported on the use of intraoperative NIRF angiography during colorectal anastomotic surgery. The NIRF angiography had to be used to assess the perfusion of the exterior surface of the bowel. Studies were found eligible for inclusion when the participants were adults, aged above 17 years, in whom open, laparoscopic or robotic anastomotic bowel surgery was performed for either benign or malignant disease. The intervention had to consist of fluorescence angiography during the anastomotic bowel surgery. If a control group was present, anastomotic bowel surgery should have been performed without the use of fluorescence imaging, also for either benign or malignant disease. Studies only describing other applications of fluorescence imaging were excluded. Conference abstracts, animal studies, case reports, technical notes, and video vignettes were not included. Studies not presenting original patient data were excluded as well. 


\section{Methodological quality appraisal}

The Methodological Index for Non-Randomized Studies (MINORS) was used to assess the methodological quality of the included articles. ${ }^{21}$ This validated quality assessment system is based on 8 items for noncomparative studies and 12 for studies with a control group.

\section{Data extraction}

All the studies were analyzed by 2 independent reviewers (JvdB and MAT). A standard form to extract the following data was used addressing the characteristics of the study (design, allocation concealment, blinding, method of randomization, withdrawals, or dropouts), participants (age, sex, baseline characteristics, indication for surgery), intervention (type of fluorescent dye, used dose, timing of administration during surgery, used fluorescence system), and the outcomes (influence on duration of surgery, type of outcome measuring, quantification of the signal, complications intra- and postoperatively).

Data from all included trials were extracted and presented in the appropriate paragraph. Baseline data are summarized as mean or proportion for continuous and categorical variables, respectively. Outcome variables are reported as absolute number and percentage stratified by intervention, for all studies combined and separately for only those studies with a control group. Differences in the proportion of anastomotic leaks between NIRF and control using all studies, and using only studies with a control group, were computed using a two-sample test for proportions.

\section{RESULTS}

The search was performed by 2 independent researchers and resulted in the inclusion of 10 articles. The search of PubMed (Medline) and EMBASE resulted in 111 citations. Checking reference lists of the selected articles identified seven additional studies. After adjusting for duplicates, 60 remained. Of these, 46 were discarded after reviewing the abstracts. The full text of the remaining 14 articles was examined in more detail. It appeared that 10 of these met the inclusion criteria. Four of these contained a comparison-group and were used for further quantitative analysis. The flowchart of the performed search and inclusion are depicted in Figure 1.

In total, the outcomes of 1328 patients were described: 894 operated with the use of NIRF imaging and 434 patients with conventional imaging. ${ }^{22-31}$ The study characteristics of the included studies can be found in Table 1. 
Number of records identified through database searching:

PubMed: 69

EMBASE: 42

Total: 111
Number of additional records

identified through other

sources:

References from found articles

$$
(n=7)
$$
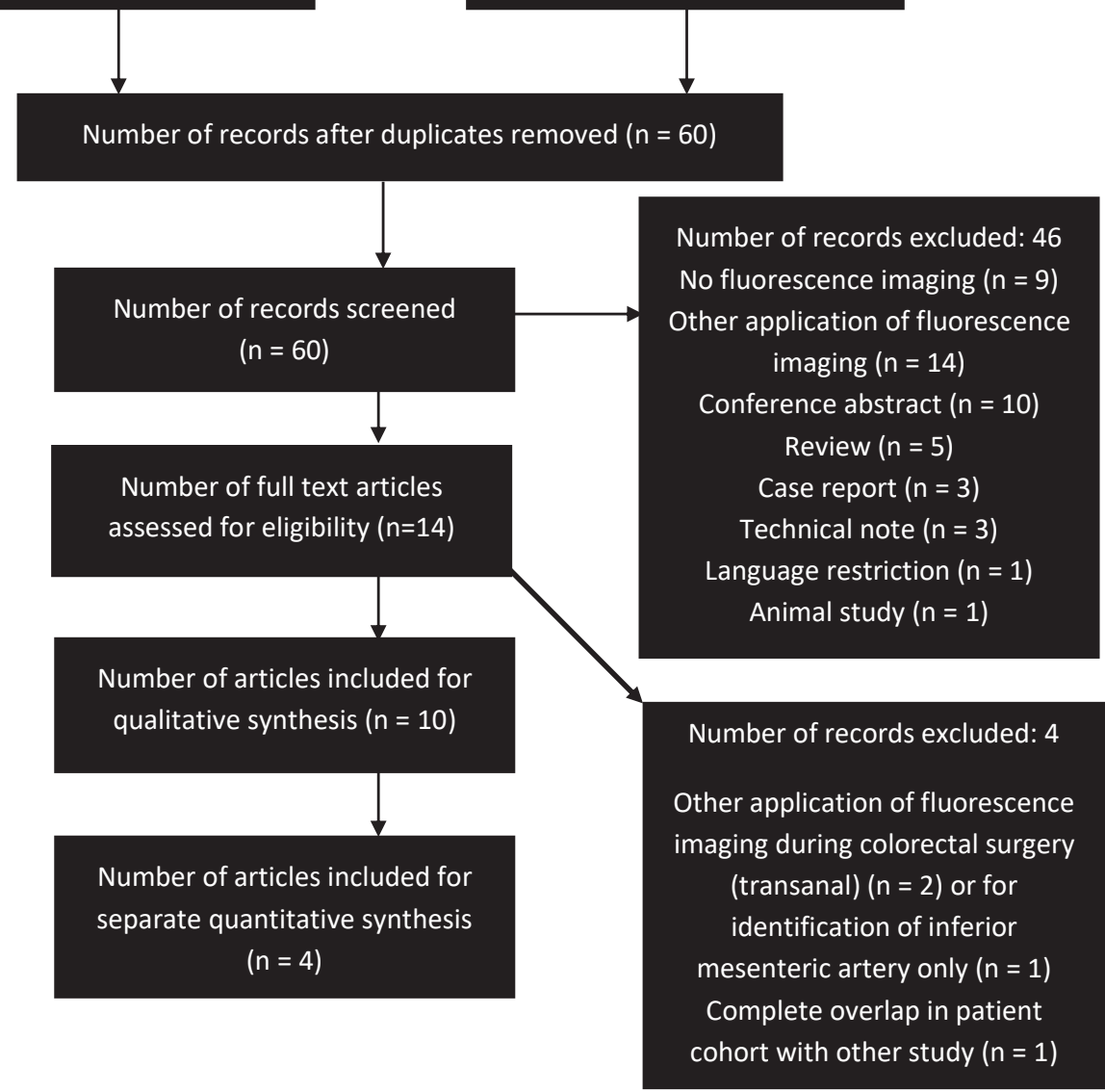

Figure 1 Flowchart of systematic search 

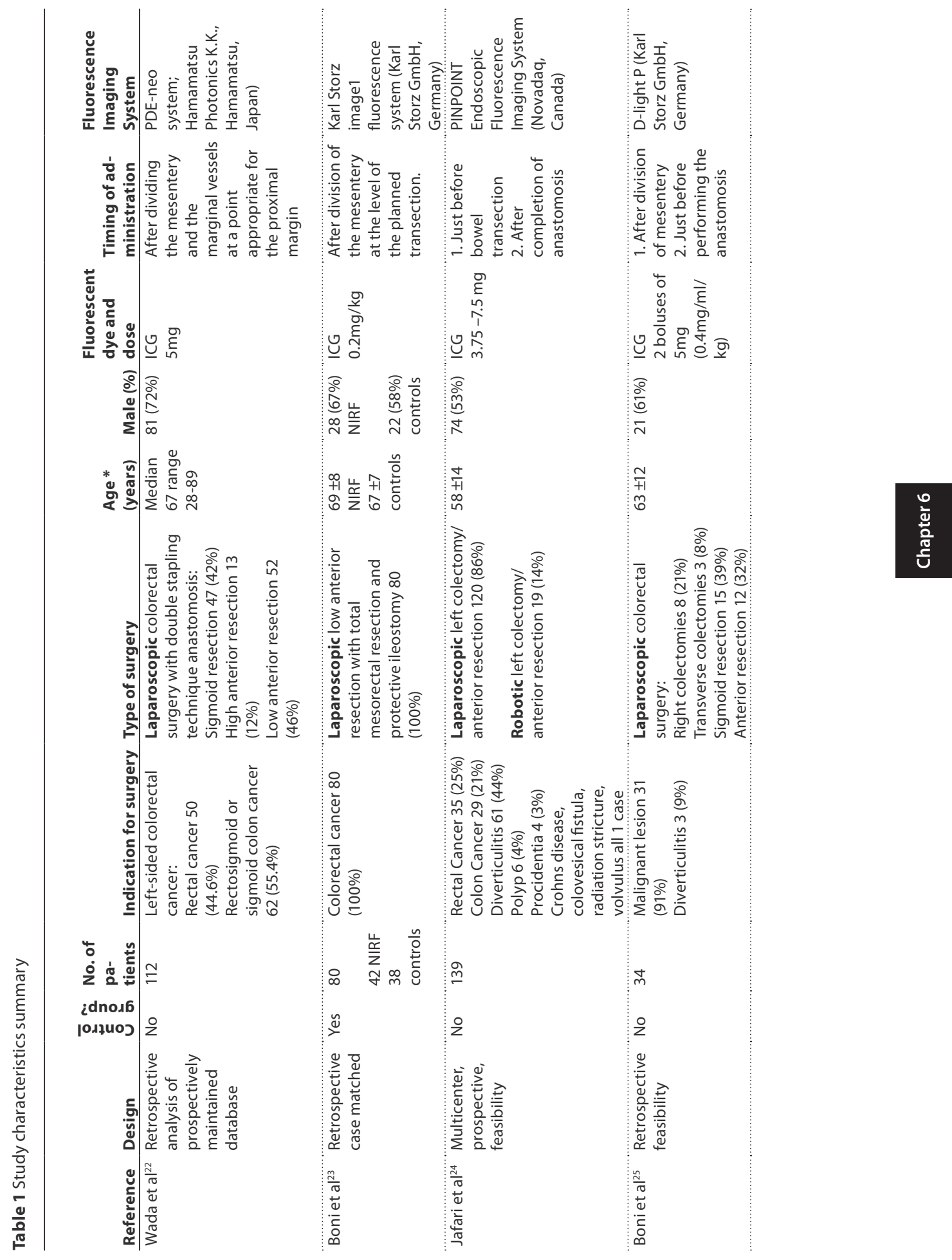


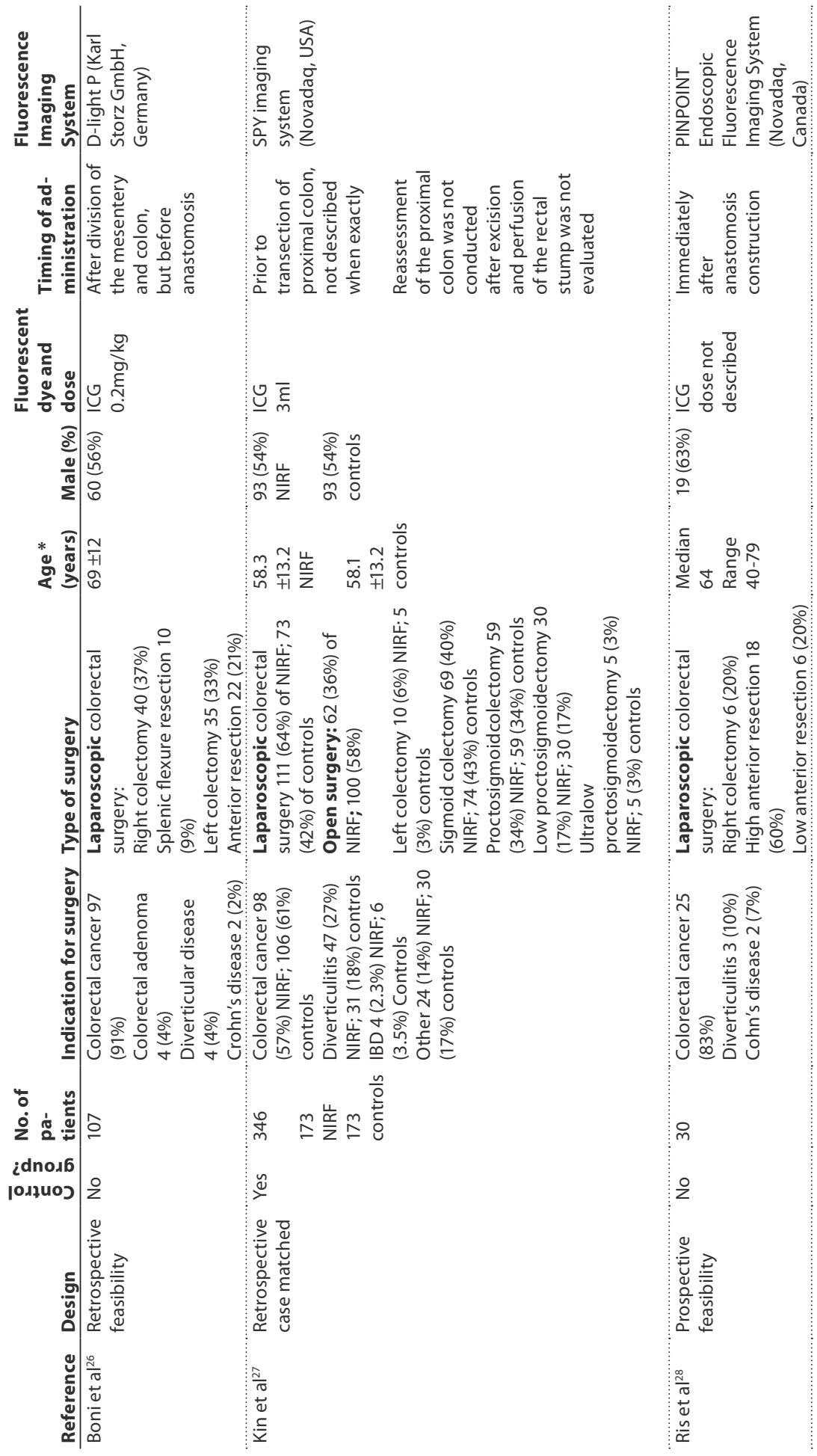




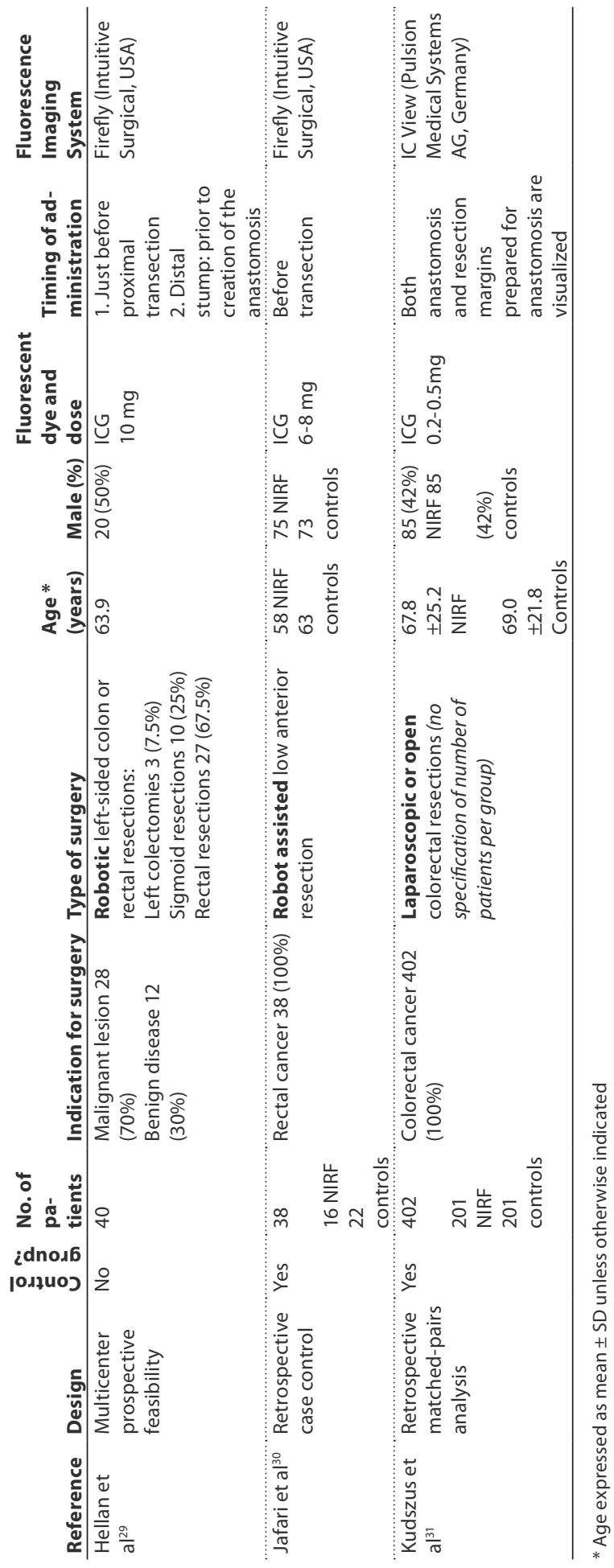




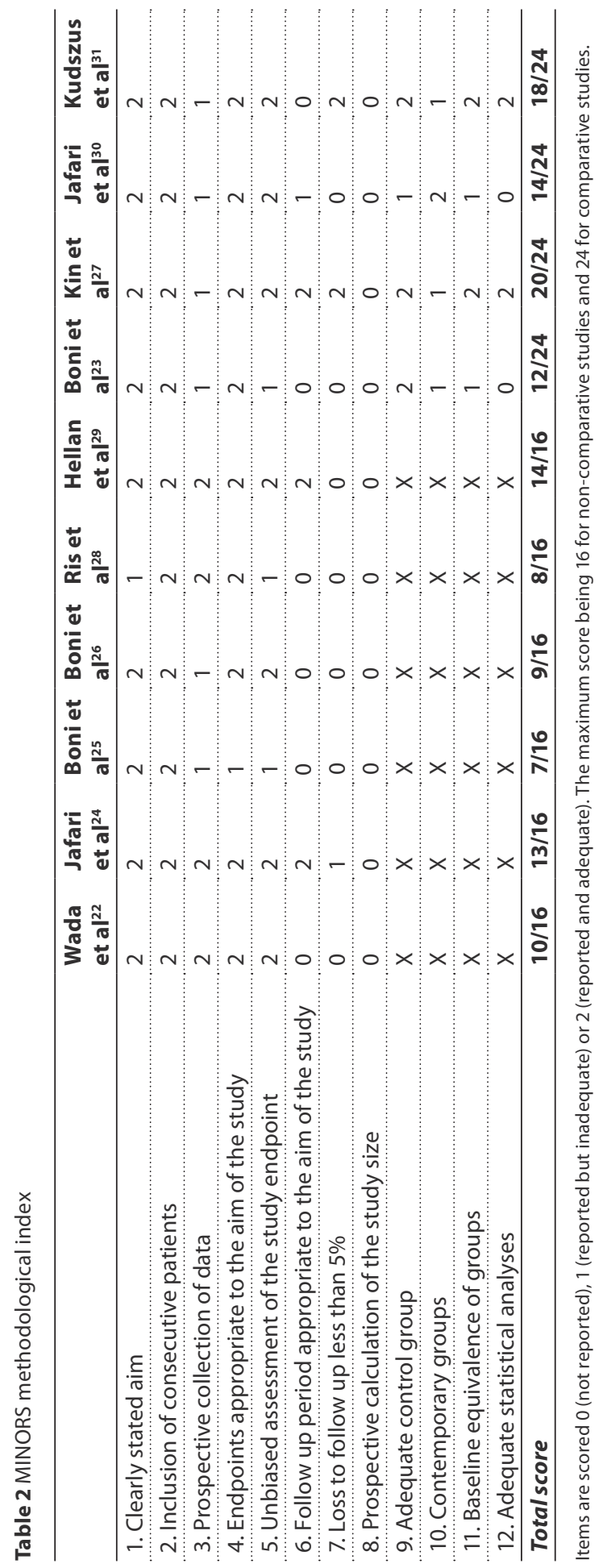




\section{Methodological quality of studies}

Since all of the included studies were nonrandomized, the risk of bias in the studies was evaluated with the MINORS methodological index.21 For studies without a control group, the maximum possible score was 16 , and 24 for the studies with a control group. The overall quality score for the assessed studies ranged between 9 and 14 (mean 10.1) for the noncomparative studies and between 12 and 20 (mean 16) for the studies with a control group (Table 2). All studies clearly stated the aim and had endpoints appropriate to this aim. Five of the studies were prospective feasibility studies, two retrospective feasibility studies, and four were retrospective matched pairs studies. The retrospective nature of most of the included studies caused an inability to control for unmeasured potential confounders between the two groups. Only three studies described loss to follow-up; the period of follow-up was unclear in most of the studies. None of the studies performed a prospective calculation of the study size. The sample size of all studies was inadequate to detect $1 \%-2 \%$ differences in anastomotic leakage rates at $80 \%$ power. ${ }^{27}$ Since from Boni et al. ${ }^{23,25,26}$ and Jafari et al. ${ }^{24,30}$ more than one article is included in this review, it was checked by the authors whether those articles were based on different patient cohorts. The authors confirmed there is no cohort overlap.

\section{Baseline characteristics of patients}

The baseline data (number of patients, mean age, gender, and body mass index) are given in Tables 3 and 4.

\section{Feasibility of the technique}

Factors contributing to the feasibility of the technique are considered to be the ability to obtain a signal illustrating perfusion, ease of use, complications due to the technique, and added surgical time. Eight of the 10 included studies make a statement on the feasibility of the technique and the ease of use $\mathrm{e}^{22-29}$; NIRF imaging of colorectal anastomosis is considered "feasible" and "readily achievable." Four of these studies illustrate this by giving the number of patients in which images that demonstrate the perfusion were successfully obtained. Jafari et al. ${ }^{24}$ acquired NIRF images in $98.5 \%$ of the patients. The imaging was not successful in 2 patients due to equipment malfunction. Boni et al..$^{25,26}$ obtain real-time images demonstrating the perfusion in all patients in both studies, as did Wada et al. ${ }^{22}$ In the study by Ris et al. ${ }^{28}$ the system failed to detect any visible fluorescence in 1 patient.

\section{Complications related to the technique}

In none of the articles complications attributable to the use of ICG were observed. 
Table 3 Table 3 Baseline data of all included studies

\begin{tabular}{llll}
\hline & NIRF & Control & Total \\
\hline Number of participants & 894 & 434 & 1328 \\
\hline Mean age & $63.4^{*}$ & 64.2 & 63.7 \\
\hline Male (\%) & $493(55)$ & $216(50)$ & $709(53)$ \\
\hline Mean BMI $\left(\mathrm{kg} / \mathrm{m}^{2}\right)$ & $26.6 \#$ & 26.3 & 26.5 \\
\hline
\end{tabular}

${ }^{*} \mathrm{Wada}^{22}$ and $\mathrm{Ris}^{28}$ are not included in this number as mean ages are not mentioned. Instead, they give ages as a median of 67 and 64 respectively

\# The studies of Boni ${ }^{25}$ and Wada ${ }^{22}$ and are not included in this figure. Boni described no BMI in the article; Wada gives BMI as median 22.8 (range $15.8-3.43 \mathrm{~kg} / \mathrm{m}^{2}$ )

Table 4 Baseline data of the four studies with a control group $23,27,30,31$

\begin{tabular}{llll}
\hline & NIRF & Control & Total \\
\hline Number of participants & 432 & 434 & 866 \\
\hline Mean age & 63.7 & 64.1 & 63.9 \\
\hline Male (\%) & $218(50.4 \%)$ & $223(51.4 \%)$ & $441(50.9 \%)$ \\
\hline Mean BMI & 26.1 & 26.3 & 26.2 \\
\hline ASA I & $10(5 \%)^{*}$ & $17(9 \%)^{*} \#$ & $27(7 \%)^{*} \#$ \\
ASA II & $78(41 \%)^{*} \#$ & $83(43 \%)^{*} \#$ & $161(42 \%)^{*} \#$ \\
ASA III & $98(52 \%)^{*} \#$ & $93(48 \%)^{*} \#$ & $191(50 \%)^{*} \#$ \\
ASA IV & $3(2 \%)^{*} \#$ & $2(1 \%)^{*} \#$ & $5(1 \%)^{*} \#$ \\
\hline Smoking & $80(35 \%)^{*}$ & $97(42 \%)^{*}$ & $177(38 \%)^{*}$ \\
\hline Preoperative chemotherapy & $78(34 \%)^{*}$ & $74(32 \%)^{*}$ & $152(33 \%)^{*}$ \\
\hdashline Indication for surgery: & & & \\
Diverticular disease & $47(11 \%)$ & $31(7 \%)$ & $78(9 \%)$ \\
Cancer & $358(83 \%)$ & $367(85 \%)$ & $725(84 \%)$ \\
IBD & $4(1 \%)$ & $6(1 \%)$ & $10(1 \%)$ \\
Other & $23(5 \%)$ & $30(7 \%)$ & $53(6 \%)$ \\
\hline
\end{tabular}

* Kusdzus et al described no ASA classification, smoking and preoperative chemotherapy; these patients are therefore not included in the overview of these parameters.

\# Boni et al described no ASA classification in their baseline characteristics; these are not included in the overview of this parameter.

\section{Added surgical time}

The added surgical time is given in five articles. ${ }^{23,28-31} \mathrm{~A}$ mean added time of 4.5 minutes to the total duration of the surgical procedure is described. Ris et al. ${ }^{28}$ noted that a shorter additional imaging time is accompanied by experience in the use of this technique. Jafari et al. ${ }^{30}$ described a mean operation time of $4.75 \mathrm{~h}$ in NIRF group, compared with $4.4 \mathrm{~h}$ in control group. Boni et al. ${ }^{23}$ report a 7-minute shorter mean operative time when using NIRF.

\section{Costs}

No comments about the additional costs of using the technique where made in the included articles ${ }^{22,23,25,27-30}$ except for Jafari et al. ${ }^{24}$ who described a cost of $\$ 167,500-223,750$ for the Pinpoint ${ }^{\mathrm{TM}}$ system, which resulted in cost per case of $\$ 999-1099$. Boni et al. ${ }^{26}$ state the technique seems to be cost effective. However, no cost evaluation to support this is given in the article. 


\section{Quantification or validation of the fluorescence signal and perfusion}

Only Wada et al.22 and Kudszus et al. ${ }^{31}$ performed an objective quantification of the fluorescence signal. One of the aims of the study by Wada et al. ${ }^{22}$ was to evaluate whether quantitative assessment of intestinal perfusion by measuring ICG signal intensity could predict postoperative outcomes such as anastomotic leakage and bowel movement recovery.

For these quantitative measurements analyzing software ROls (Hamamatsu Photonics) was used to assess the pixel intensity. A time curve of this fluorescence intensity was made and fluorescence difference between maximum and baseline was measured, as were time from first fluorescence to maximum signal, time from first fluorescence to half of the maximum signal, and the slope of this graph. For the regions of interest at the transection line, the midpoint between the mesenteric and antimesenteric sites was chosen. The maximum fluorescence intensity turned out to be lower in the 5 patients with anastomotic leakage in this study, compared to the patients in which no anastomotic leakage occurred.

Also, Kudszus et al. ${ }^{31}$ used an objective quantification of the fluorescence signal and perfusion. For this, IC-Calc ${ }^{\circledast}$ software was used. With this software, the perfusion index is calculated (i.e., the perfusion between different sites) and curves are plotted. ${ }^{32}$ The authors show the curves but unfortunately no cutoff value of fluorescence intensity for adequate perfusion is calculated or given, neither is the fluorescence intensity linked to the risk for anastomotic leakage. All other included studies only use a subjective assessment of the fluorescence signal. The surgeons' opinion is used, ${ }^{23,25,27,29,30}$ or that of the whole surgical team (main surgeon, main assistant, fellow in minimally invasive surgery, and the assisting resident $)^{26}$ to assess whether the fluorescent signal was enough to maintain the planned resection point. In these studies, no grading was used other than "adequate/sufficient" or "inadequate/insufficient." Jafari et al. ${ }^{24}$ and Ris et al. ${ }^{28}$ divided the subjective assessment in three categories, namely "optimal, adequate, or inadequate" and "good, average, and bad" respectively. Neither study describes when a revision of the surgical plan will take place based on this classification. Moreover, Ris et al..$^{28}$ graded the quality of perfusion in all patients as "Good," while Jafari et al. ${ }^{24}$ doesn't report the results of this grading.

\section{Added value of the technique}

The outcomes regarding perioperative decision making for all studies are summarized in Table 5, for the studies with a control group separately in Table 6.

\section{Influence of NIRF angiography on intraoperative decision making}

In total, surgical plan was changed in 97 (10.8\%) cases after NIRF, of which in 90 the proximal resection margin was initially considered insufficient with NIRF. In this group, anastomotic leakage occurred in 8 patients (8.9\%), compared to 23 anastomotic leakages $(2.8 \%)$ in the NIRF patients with initially good fluorescent signal. In 7 NIRF patients, initial clinical impression of malperfusion was not confirmed by NIRF and resection was not extended. 
Table 5 Added value of the technique based on all included studies

\begin{tabular}{|c|c|c|c|}
\hline & $\begin{array}{l}\text { NIRF } \\
(n=894)\end{array}$ & $\begin{array}{l}\text { Control } \\
(n=434)\end{array}$ & $\begin{array}{l}\text { Total } \\
(n=1328)\end{array}$ \\
\hline Revision of surgical plan & $97(10.8 \%)$ & $1(0.2 \%)$ & $98(7.4 \%)$ \\
\hline $\begin{array}{l}\text { Anastomotic leak after revision } \\
\text { of surgical plan }\end{array}$ & $8(8.3 \%)$ & 0 & $8(8.2 \%)$ \\
\hline Total number of anastomotic leaks & $31(3.5 \%)^{*}$ & $32(7.4 \%)^{*}$ & $63(4.7 \%)$ \\
\hline
\end{tabular}

*P-value: 0.002

Table 6 Added value of the technique based on the four studies with a control group $23,27,30,31$

\begin{tabular}{llll}
\hline & $\begin{array}{l}\text { NIRF } \\
(\mathbf{n = 4 3 2 )}\end{array}$ & $\begin{array}{l}\text { Control } \\
(\mathbf{n = 4 3 4 )}\end{array}$ & $\begin{array}{l}\text { Total } \\
(\mathbf{n = 8 6 6 )}\end{array}$ \\
\hline Revision of surgical plan & $47(10.9 \%)$ & $1(0.2 \%)$ & $48(5.5 \%)$ \\
\hline $\begin{array}{l}\text { Anastomotic leak after revision } \\
\text { of surgical plan }\end{array}$ & $2(4.3 \%)$ & 0 & $1(1.9 \%)$ \\
\hdashline & & & \\
\hline Total number of anastomotic leaks & $21(4.9 \%)$ & $32(7.4 \%)$ & $53(6.1 \%)$ \\
\hline
\end{tabular}

In the studies with a control group, the surgical plan was changed in 47 cases (10.9\%) of the NIRF group and in 1 case of the control group. Of these, in 41 patients the proximal resection margin was considered insufficiently vascularized and therefore extended to well perfused tissue. In the other 6 patients, a clinical impression of malperfusion of the resection margins was not confirmed by NIRF angiography and therefore the resection was not extended. 23,27,30,31 The change of plan therefore consisted of neglecting the clinical impression and instead following the NIRF imaging. In 1 of the 41 patients of the first group, two anastomotic leakages occurred. No further information about this anastomotic leakage was provided. ${ }^{27}$ In the second group of 6 patients, no anastomotic leakage occurred. ${ }^{30,31}$

In the studies without a control group, the surgical plan was revised in 50 patients $(10.8 \%)$ after NIRF imaging. In 49 of these, a revision was performed of the proximal transection site. Six of these patients (12\%) developed an anastomotic leak, at days 8, 15, 17, and 40.22,29

The other patients in whom the plan was revised, a clinically malperfused anastomosis was left unchanged because the NIRF angiography revealed good perfusion. No anastomotic leakage occurred in these patients.

\section{Anastomotic leakage and other complications}

The definition of anastomotic leakage differed between the studies. Wada et al. ${ }^{22}$ used the following definition: "Anastomotic leakage was defined as any disruption of the anastomosis that was confirmed by digital rectal examination, sigmoidoscopy, and radiographic examination (e.g., extravasion of endoluminally administered water-soluble contrast enema, abscess at the level of anastomosis and fluid/air bubbles surrounding the anastomosis). Only symptomatic AL was included." Kin et al. ${ }^{27}$ used a broader definition and defined an anastomotic leak as "at least one of the following criteria: 1. An anastomotic defect noted on physical examination; 2 . An 
Table 7 Complications other than anastomotic leakage described by Wada et $\mathrm{al}^{22}$, Boni et $\mathrm{al}^{23}$, Jafari et al ${ }^{24}$, Boni et $\mathrm{al}^{26}$, Hellan et $\mathrm{al}^{29}$ and Jafari et $\mathrm{al}^{30}$

\begin{tabular}{llll}
\hline & NIRF (n= 456) & Control (n= 60) & Total (n= 516) \\
\hline Ileus (\%) & $22(4.8)$ & $6(10)$ & $28(5.4)$ \\
\hline Urinary retention (\%) & $10(2.2)$ & $5(8.3)$ & $15(2.9)$ \\
\hline Wound infection (\%) & $7(1.5)$ & $3(5)$ & $10(1.9)$ \\
\hline Urinary tract infection (\%) & $14(3.1)$ & $7(11.7)$ & $21(4.1)$ \\
\hline Requiring blood transfusion (\%) & $10(2.2)$ & $2(3.3)$ & $12(2.3)$ \\
\hline Pulmonary complications (\%) & $16(3.5)$ & $6(10)$ & $22(4.2)$ \\
\hline Fever (\%) & $4(0.9)$ & 0 & $4(0.8)$ \\
\hline Sepsis (\%) & $1(0.2)$ & $3(13.6)$ & $4(0.8)$ \\
\hline Incisional Hernia (\%) & $3(0.7)$ & 0 & $3(0.6)$ \\
\hline Rectal bleeding (\%) & $2(0.4)$ & 0 & $2(0.4)$ \\
\hdashline Pelvic abcess (\%) & $2(0.4)$ & 0 & $2(0.4)$ \\
Bleeding at anastomotic site (\%) & $1(0.2)$ & 0 & $1(0.2)$ \\
Other * (\%) & $26(5.7)$ & $5(8.3)$ & $31(6.0)$ \\
\hline Total (\%) & $\mathbf{1 1 0 ( 3 2 . 0 )}$ & $\mathbf{3 7 ( 6 1 . 7 )}$ & $\mathbf{1 4 7 ( 3 6 . 4 )}$ \\
\hline
\end{tabular}

* Thrombosis left renal artery, pancreatic fistula, atrial fibrillation, $\mathrm{C}$ difficile colitis acute renal failure, peristomal hernia, obstruction at stoma and death by suicide: all occurred once, other complications where described as 'other', without further specification.

anastomotic defect confirmed in the operation room; 3. An anastomotic defect seen on proctoscopy; 4. Radiologic evidence of a leak consisting of either a defect in the anastomosis and an adjacent fluid collection, or stranding or the extravasion of rectal contrast into the extraluminal space; or 5. Clinical evidence of a leak such as feculent output from a pelvic drain." The other included articles did not give a clear definition of anastomotic leakage. ${ }^{23-26,28-31}$

Tables 5 and 6 show a summary of the occurrence of anastomotic leaks. A total of 63 anastomotic leakages occurred in the 10 included studies (NIRF group $\mathrm{n}=31$; control group $n=32$ ). This means that $3.5 \%$ (31 out of 894 ) of all NIRF patients and $7.4 \%$ (32 out of 434) of all control patients developed an anastomotic leakage ( $P$-value for difference $=.002$ ). If only considering the studies with a control group, a 4.9\% (21 out of 432) leakage rate in the NIRF group was found versus $7.4 \%$ (32 out of 434 ) in the control group ( $P=.123$ ).

Other complications that occurred were inconsistently reported in the included articles. Boni et al.,r ${ }^{25}$ Kin et al. ${ }^{27}$ and Kudszus et al. ${ }^{31}$ reported no complications other than anastomotic leakage. Ris et al. ${ }^{28}$ described only the grade of the occurred complications (grades I-IV Clavien-Dindo classification): all seven complications were grades $1-3$. In the remaining six studies detailed information was given about the complications (Table 7). lleus, urinary retention, urinary tract infection, pulmonary complications and wound infection were the most common complications, occurring relatively more in the control group. ${ }^{22,24,26,29,30}$ 


\section{DISCUSSION}

The aim of this study was to investigate the feasibility of the use of NIRF angiography during anastomotic bowel surgery and determining the added value of the technique. Regarding the latter, attention was paid to whether the fluorescent signal was quantified or validated in any way.

Based on the included studies, it can be concluded that the use of fluorescence angiography in anastomotic bowel surgery is feasible. No complications attributable to the use of the technique were recorded. This makes the technique easily applicable in daily practice. Only few problems were encountered in its application, as system failure occurred only in 3 patients. This may be due to the relative inexperience of the team with this novel approach. A learning curve in the use of this technique might be present. Therefore, it can be expected that such system failure will be less frequent when this technique is used more often. It cannot be ruled out that a learning curve effect has been of influence on other observed differences between studies.

Minimal additional time is described in most articles. Jafari et al. ${ }^{30}$ describe an average 21 minutes extra operation time in NIRF group. In these series, the decision to use NIRF angiography was at the discretion of the attending surgeon. Correspondence with the author reveals that the ICG imaging was reserved for more complex surgeries, with a longer duration for that reason. The NIRF angiography itself was thought to add maximally 10 minutes to the operation time.

Apart from safety and ease of use, the ability of the technique to change the surgical plan with positive outcome on operative results reflects the value of this technique. The overall rate of anastomotic leakages in the included studies was 4.7\% (63 of 1328 patients); this percentage is comparable to what can be found in the literature. ${ }^{33-38}$ Fluorescence angiography changed the surgical plan in $10.8 \%$ of all NIRF cases. Anastomotic leakage was less prevalent in the NIRF group (3.4\%) than in the control group (7.4\%). This may be considered an indication of the benefit of the technique adding in improving the surgeons' ability to detect areas of poor blood supply. This consideration is supported by a pig study by Diana et al., ${ }^{39}$ that showed that NIRF angiography allows qualitative assessment of the bowel perfusion. Yet, based on the still limited quality of the current research no solid evidence is provided to state that NIRF imaging significantly reduces the incidence of anastomotic leaks. Likewise, the studies comparing the NIRF technique with a control group of conventional imaging, still lack the strength and the evidence. Kin et al. ${ }^{27}$ published the biggest study comparing the NIRF angiography with a control group. Their conclusion is that the benefit of NIRF angiography is equivocal. However, in this study only the proximal bowel was assessed and the perfusion of the rectal stump was not evaluated. This could explain the absence of difference between the two groups, as a malperfusion of the rectal stump may have been missed, leaving the potential advantage of the NIRF technique 
underestimated. This is also identified as a limitation by the authors. The same technique was used by Wada et al. ${ }^{22}$ and Boni et al. ${ }^{26}$

An anastomotic leak rate of $8.9 \%$ was found after revision of the surgical plan, compared to $2.8 \%$ in the patients with initial good fluorescence signal. This could mean that a good fluorescence signal is predicting a good outcome, while a lesser fluorescent signal could mean a higher risk of anastomotic leakage, whether or not the transection line is moved.

In this review, attention was given to whether the signal was quantified or validated in an objective manner or only subjectively assessed. Unfortunately, in only two studies this was attempted. As described, Kudszus et al. ${ }^{31}$ used an objective measurement using IC-Calc. Although this method is validated in rabbits, ${ }^{32}$ in their present study, no cutoff value was given on which revision of the resection margin was based. Wada et al. ${ }^{22}$ used other software, namely "ROIs" (Hamamatsu Photonics K.K.) to measure the fluorescence intensity. In this study, four parameters were measured, namely difference in fluorescence between maximum signal and baseline, time from first fluorescence to maximum signal, time from first fluorescence to half of maximum signal, and the slope; fluorescence difference divided by the time until maximum fluorescence. A lower maximum fluorescence signal was observed in the patients who developed anastomotic leakage compared to the other patients. Also, a less steep slope appeared predictive for anastomotic leakage. The time until maximum fluorescence and time to half of maximum fluorescence appeared not to be predictive for anastomotic leakage.

In the other studies, the signal was subjectively assessed by the surgeon or his team. The assessment was mostly binary: considered sufficiently or not. ${ }^{23,25-27,29,30}$ Jafari et al. ${ }^{24}$ and Ris et al. ${ }^{28}$ divided the subjective assessment in three categories, namely "optimal, adequate, or inadequate" and "good, average, and bad" respectively. However, no specific relation of the three categories on peroperative decision making and outcome was described in their articles.

Another possible method to objectively assess the NIRF signal is ad hoc imaging software (VR-render) as described by Diana et al. ${ }^{40}$ This software constructs a cartography of the perfused area based on the fluorescence time-to-peak and makes a real-time overlay of this cartography on the laparoscopic image. This technique has not yet been used in humans, but was validated in an animal study by Diana et al. ${ }^{40}$

Other methods described in literature are the postoperative measurement of the Target to Background Ratio using the fluorescence intensity using OsiriX (Pixmeo, Geneva, Switzerland) ${ }^{41}$; and the Signal to Noise ratio using ImageJ software (National Institutes of Health, Washington, DC). ${ }^{42}$ Unfortunately, these techniques are used for postoperative measurement and not applicable yet in the intraoperative setting. Further studies should focus on this validation. It may be expected that this will lead to more accurate recognition of imperfect perfusion levels that will contribute to the chance of anastomotic leakage and therefore to better use of the potential of the NIRF technique. 
In the control group, apart from more anastomotic leakages, also other complications occurred more often $(61 \%$ in the control group as compared to $32 \%$ in the intervention group). It is unclear from the included articles whether this higher complication rate is due to the higher rate of anastomotic leakage. ${ }^{23,30}$

In the present review, no statement on the cost or savings due of the technique can be given, as this is not studied in the majority of the included articles. Yet, this is an interesting result to be included in future research. The technique has the potential to reduce anastomotic leaks. A rough estimate of its cost is between $\$ 10,000$ and $\$ 50,000$ more per patient with an anastomotic leak as reported in current literature. ${ }^{8,43,44}$ Any extra time still spent with its application may decrease in more routine use. And further, the fluorescence imaging mode is or will soon be commercially available in frequently used endoscopic systems, eliminating the need for obtaining a separate and therefore costly extra set of equipment. This leaves only about $\$ 80$ for the required ICG as extra costs. Based on the above given estimate of cost per leak, this means that in every 125-625 (10,000/80-50,000/80) patients operated with NIRF 1 anastomotic leak should be avoided for this technique to be cost effective.

This review should be viewed with consideration based on the following. First, the included studies were all of limited quality. All the included studies were nonrandomized, and the studies with a control group were retrospective case matched studies. The case matching could prevent bias by selecting comparable groups when taking all risk factors for anastomotic leakage in account. However, in the study by Jafari et al. ${ }^{30}$ the controls were selected from cases performed in the same period and it was not described why those patients did not undergo fluorescence imaging. This particular reason could be of influence on the outcome. Boni et al., ${ }^{23}$ Kin et al., ${ }^{27}$ and Kudszus et al. ${ }^{31}$ all selected cases from the period before the introduction of the NIRF technique. Although the articles discussed the lack of difference in treatment between the two periods of time, minor changes might still be present and could therefore have had an influence on the outcome. Furthermore, the retrospective nature prohibits correction for unmeasured confounders between the two groups. Another limitation is the inclusion of mostly small studies with an unclear follow-up period and lack of prospective sample size calculation. Because of the inconsistent reporting and the small number of patients no conclusions on the occurrence of complications can be made.

In summary, fluorescent imaging for assessment of bowel perfusion in colorectal resectional surgery is feasible and easy to use and based on the reports so far, holds great potential. All authors are positive about its added value over the usual clinical assessment methods. Nevertheless, data are still limited and results on quantification and validation of the signal are scarce. Further studies are needed to validate the technique and establish its contribution to the prevention of anastomotic leakage. 


\section{REFERENCES}

1. Leahy J, Schoetz D, Marcello P, Read T, Hall J, Roberts P, et al. What is the risk of clinical anastomotic leak in the diverted colorectal anastomosis? J Gastrointest Surg 2014; 18:18121816.

2. Sultan R, Chawla T, Zaidi M. Factors affecting anastomotic leak after colorectal anastomosis in patients without protective stoma in tertiary care hospital. J Pak Med Assoc 2014;64:166-170.

3. Morse BC, Simpson JP, Jones YR, Johnson BL, Knott BM, Kotrady JA. Determination of independent predictive factors for anastomotic leak: Analysis of 682 intestinal anastomoses. Am J Surg 2013;206:950-955; discussion 5-6.

4. Leichtle SW, Mouawad NJ, Welch KB, Lampman RM, Cleary RK. Risk factors for anastomotic leakage after colectomy. Dis Colon Rectum 2012;55:569-575.

5. Ruggiero R, Sparavigna L, Docimo G, Gubitosi A, Agresti M, Procaccini E, et al. Post-operative peritonitis due to anastomotic dehiscence after colonic resection. Multicentric experience, retrospective analysis of risk factors and review of the literature. Ann Ital Chir 2011;82:369-375.

6. Buchs NC, Gervaz P, SecicM, Bucher P,Mugnier-Konrad B, Morel P. Incidence, consequences, and risk factors for anastomotic dehiscence after colorectal surgery: A prospective monocentric study. Int J Colorectal Dis 2008;23:265-270.

7. Midura EF, Hanseman D, Davis BR, Atkinson SJ, Abbott DE, Shah SA, et al. Risk factors and consequences of anastomotic leak after colectomy: A national analysis. Dis Colon Rectum 2015;58:333-338.

8. Turrentine FE, Denlinger CE, Simpson VB, Garwood RA, Guerlain S, Agrawal A, et al. Morbidity, mortality, cost, and survival estimates of gastrointestinal anastomotic leaks. J Am Coll Surg 2015;220:195-206.

9. Nachiappan S, Faiz O. Anastomotic leak increases distant recurrence and long-term mortality after curative resection for colonic cancer. Ann Surg 2014;259:930-938.

10. McDermott FD, Heeney A, Kelly ME, Steele RJ, Carlson GL, Winter DC. Systematic review of preoperative, intraoperative and postoperative risk factors for colorectal anastomotic leaks. $\mathrm{Br}$ J Surg 2015;102:462-479.

11. Karliczek A, Harlaar NJ, Zeebregts CJ, Wiggers T, Baas PC, van Dam GM. Surgeons lack predictive accuracy for anastomotic leakage in gastrointestinal surgery. Int J Colorectal Dis 2009;24:569_ 576.

12. Nachiappan S, Askari A, Currie A, Kennedy RH, Faiz O. Intraoperative assessment of colorectal anastomotic integrity: A systematic review. Surg Endosc 2014;28:2513-2530.

13. Pommergaard HC, Achiam MP, Burcharth J, Rosenberg J. Impaired blood supply in the colonic anastomosis in mice compromises healing. Int Surg 2015;100:70-76.

14. Chachkhiani I, Gurlich R, Maruna P, Frasko R, Lindner J. The postoperative stress response and its reflection in cytokine network and leptin plasma levels. Physiol Res 2005;54:279-285.

15. Wu JH, Rong ZX, Zhu DJ, Chen XW, Ren BJ. [Laparoscopic anterior resection of rectal carcinoma with preservation of the left colonic artery]. Nan Fang Yi Ke Da Xue Xue Bao 2009;29:1249-1250.

16. Karliczek A, Benaron DA, Zeebregts CJ, Wiggers T, van Dam GM. Intraoperative ischemia of the distal end of colon anastomoses as detected with visible light spectroscopy causes reduction of anastomotic strength. J Surg Res 2009;152:288-295.

17. Sherwinter DA. Transanal near-infrared imaging of colorectal anastomotic perfusion. Surg Laparosc Endosc Percutan Techn 2012;22:433-436.

18. Flower RW, Hochheimer BF. Indocyanine green dye fluorescence and infrared absorption choroidal angiography performed simultaneously with fluorescein angiography. Johns Hopkins Med J 1976;138:33-42.

19. Penha FM, Rodrigues EB, Maia M, Meyer CH, Costa Ede P, Dib E, et al. Biochemical analysis and decomposition products of indocyanine green in relation to solvents, dye concentrations and laser exposure. Ophthalmologica 2013; 230(Suppl 2):59-67. 
20. Cahill R, Ris F, Mortensen N. Near-infrared laparoscopy for real-time intra-operative arterial and lymphatic perfusion imaging. Colorectal Dis 2011;13(Suppl 7):12-17.

21. Slim K, Nini E, Forestier D, Kwiatkowski F, Panis Y, Chipponi J. Methodological index for nonrandomized studies (MINORS): Development and validation of a new instrument. ANZ J Surg 2003;73:712-716.

22. Wada T, Kawada K, Takahashi R, Yoshitomi M, Hida K, Hasegawa S, et al. ICG fluorescence imaging for quantitative evaluation of colonic perfusion in laparoscopic colorectal surgery. Surg Endosc 2017 [Epub ahead of print]; doi:10.1007/s00464-017-5475-3.

23. Boni L, Fingerhut A, Marzorati A, Rausei S, Dionigi G, Cassinotti E. Indocyanine green fluorescence angiography during laparoscopic low anterior resection: Results of a casematched study. Surg Endosc 2017;31:1836-1840.

24. Jafari MD, Wexner SD, Martz JE, McLemore EC, Margolin DA, SherwinterDA, et al. Perfusion assessment in laparoscopic left-sided/anterior resection (PILLAR II): A multi-institutional study. J Am Coll Surg 2015;220:82.e1-92.e1.

25. Boni L, David G, Mangano A, Dionigi G, Rausei S, Spampatti S, et al. Clinical applications of indocyanine green (ICG) enhanced fluorescence in laparoscopic surgery. Surg Endosc 2015;29:2046-2055.

26. Boni L, David G, Dionigi G, Rausei S, Cassinotti E, Fingerhut A. Indocyanine green-enhanced fluorescence to assess bowel perfusion during laparoscopic colorectal resection. Surg Endosc 2016;30:2736-2742.

27. Kin C, Vo H, Welton L, Welton M. Equivocal effect of intraoperative fluorescence angiography on colorectal anastomotic leaks. Dis Colon Rectum 2015;58:582-587.

28. Ris F, Hompes R, Cunningham C, Lindsey I, Guy R, Jones O, et al. Near-infrared (NIR) perfusion angiography in minimally invasive colorectal surgery. Surg Endosc 2014;28:2221-2226.

29. Hellan M, Spinoglio G, Pigazzi A, Lagares-Garcia JA. The influence of fluorescence imaging on the location of bowel transection during robotic left-sided colorectal surgery. Surg Endosc 2014;28:1695-1702.

30. Jafari MD, Lee KH, Halabi WJ, Mills SD, Carmichael JC, Stamos MJ, et al. The use of indocyanine green fluorescence to assess anastomotic perfusion during robotic assisted laparoscopic rectal surgery. Surg Endosc 2013;27:3003-3008.

31. Kudszus S, Roesel C, Schachtrupp A, Hoer JJ. Intraoperative laser fluorescence angiography in colorectal surgery: A noninvasive analysis to reduce the rate of anastomotic leakage. Langenbecks Arch Surg 2010;395:1025-1030.

32. Toens C, Krones CJ, Blum U, Fernandez V, Grommes J, Hoelzl F, et al. Validation of IC-VIEW fluorescence videography in a rabbit model of mesenteric ischaemia and reperfusion. Int J Colorectal Dis 2006;21:332-338.

33. Kingham TP, Pachter HL. Colonic anastomotic leak: Risk factors, diagnosis, and treatment. J Am Coll Surg 2009;208: 269-278.

34. Chambers WM, Mortensen NJ. Postoperative leakage and abscess formation after colorectal surgery. Best Pract Res Clin Gastroenterol 2004;18:865-880.

35. Brown SR, Mathew R, Keding A, Marshall HC, Brown JM, Jayne DG. The impact of postoperative complications on long-term quality of life after curative colorectal cancer surgery. Ann Surg 2014;259:916-923.

36. Marinello FG, Baguena G, Lucas E, Frasson M, Hervas D, Flor-Lorente B, et al. Anastomotic leaks after colon cancer resections: Does the individual surgeon matter? Colorectal Dis 2016;18:624625.

37. Shekarriz H, Eigenwald J, Shekarriz B, Upadhyay J, Shekarriz J, Zoubie D, et al. Anastomotic leak in colorectal surgery: Are 75\% preventable? Int J Colorectal Dis 2015;30:1525-1531.

38. Yun JA, Cho YB, Park YA, Huh JW, Yun SH, Kim HC, et al. Clinical manifestations and risk factors of anastomotic leakage after low anterior resection for rectal cancer. ANZ J Surg 2015 [Epub ahead of print]; doi:10.1111/ans.13143. 
39. 39. Diana M, Agnus V, Halvax P, Liu YY, Dallemagne B, Schlagowski Al, et al. Intraoperative fluorescence-based enhanced reality laparoscopic real-time imaging to assess bowel perfusion at the anastomotic site in an experimental model. Br J Surg 2015;102:e169-e176.

40. Diana M, Noll E, Diemunsch P, Dallemagne B, Benahmed MA, Agnus V, et al. Enhanced-reality video fluorescence: A real-time assessment of intestinal viability. Ann Surg 2014;259:700-707.

41. Schols RM, Lodewick TM, Bouvy ND, van Dam DA, Meijerink WJ, van Dam GM, et al. Nearinfrared fluorescence laparoscopy of the cystic duct and artery in pigs: Performance of a preclinical dye. J Laparoendosc Adv Surg Tech A 2014;24:318-322.

42. Zhu B, Rasmussen JC, Sevick-Muraca EM. A matter of collection and detection for intraoperative and noninvasive near-infrared fluorescence molecular imaging: To see or not to see? Med Phys 2014;41:022105.

43. Hammond J, Lim S, Wan Y, Gao X, Patkar A. The burden of gastrointestinal anastomotic leaks: An evaluation of clinical and economic outcomes. J Gastrointest Surg 2014;18:1176-1185.

44. Ashraf SQ, Burns EM, Jani A, Altman S, Young JD, Cunningham C, et al. The economic impact of anastomotic leakage after anterior resections in English NHS hospitals: Are we adequately remunerating them? Colorectal Dis 2013;15:e190-e198. 
CHAPTER 7

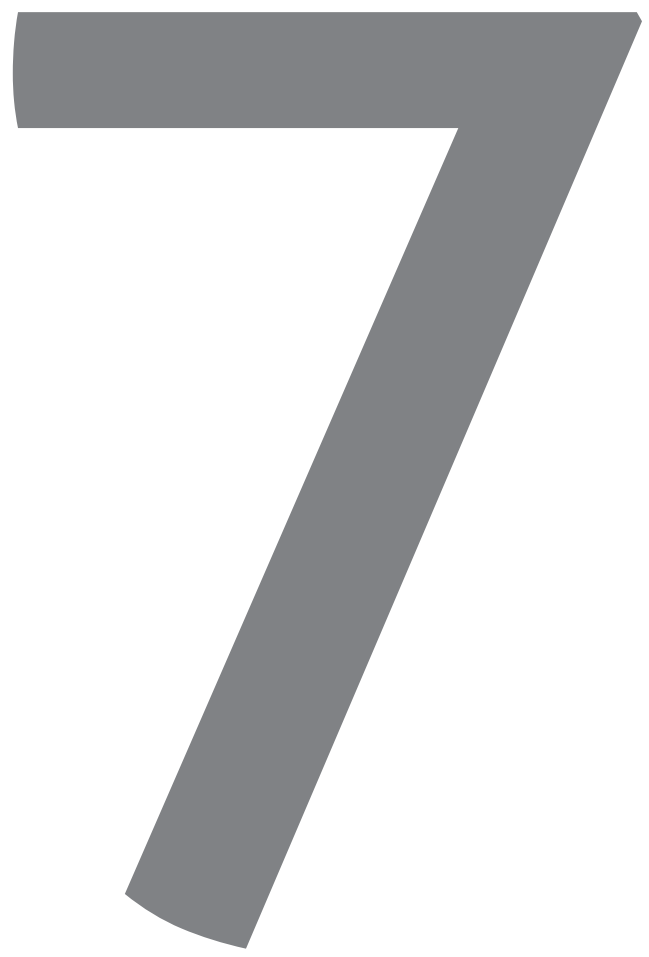




\section{Near-Infrared fluorescence imaging in anastomotic surgery: is fluorescence intensity related to anastomotic leakage?}

Jacqueline van den Bos, Audrey C.H.M. Jongen, Jarno Melenhorst,

Stephanie O. Breukink, Kaatje Lenaerts, Rutger M. Schols, Nicole D. Bouvy, Laurents P.S. Stassen.

Surgical Endoscopy 2019 [Epub Ahead of print] 


\section{ABSTRACT Objective}

Near-infrared fluorescence (NIRF) imaging using indocyanine green (ICG) might help reduce anastomotic leak-age (AL) after colorectal surgery. This pilot study aims to analyze whether a relation exists between measured fluorescence intensity (FI) and postoperative inflammatory markers of $A L, C$-reactive protein (CRP), Intestinal fatty-acid binding protein (I-FABP), and calprotectin, to $\mathrm{AL}$, in order to evaluate the potential of FI to objectively predict AL.

\section{Methods}

Patients scheduled for anastomotic colorectal cancer surgery were eligible for inclusion in this prospective pilot study. During surgery, at three time points (after bowel devascularization; before actual transection; after completion of anastomosis) a bolus of 0.2 $\mathrm{mg} / \mathrm{kg}$ ICG was administered intravenously for assessment of bowel perfusion. FI was scored in scale from 1 to 5 based on the operating surgeon's judgment ( 1 = no fluorescence visible, 5 = maximum fluorescent signal). The complete surgical procedure was digitally recorded. These recordings were used to measure FI postoperatively using OsiriX imaging software. Serum CRP, I-FABP, and calprotectin values were determined before surgery and on day 1, 3, and 5 postoperative; furthermore, the occurrence of $A L$ was recorded.

\section{Results}

Thirty patients ( $\mathrm{n}=19$ males; mean age 67 years; mean BMI 27.2) undergoing either laparoscopic or robotic anastomotic colorectal surgery were included. Indication for surgery was rectal $(n=10)$, rectosigmoid $(n=2)$, sigmoid $(n=10)$, or more proximal colon carcinomas $(n=8)$. Five patients $(16.7 \%)$ developed $A L(n=2(6.6 \%)$ grade $C$ according to the definition of the International Study group of Rectal Cancer). In patients with $A L$, the maximum fluorescence score was given less often $(P=0.02)$ and a lower $F I$ compared to background FI was measured at 1st assessment $(P=0.039)$. However, no relation between $\mathrm{FI}$ and postoperative inflammatory parameters could be found.

\section{Conclusion}

Both subjective and measured FI seem to be related to AL. In this study, no relation between $\mathrm{Fl}$ and inflammatory serum markers could yet be found. 


\section{INTRODUCTION}

Anastomotic leakage $(\mathrm{AL})$ is the most feared complication in colorectal (cancer) surgery. The severity of leakage may vary from a contained peri-anastomotic fluid collection to generalized peritonitis. Furthermore, long-term morbidity including stricture formation, bowel dysfunction, and an increased chance of locoregional cancer recurrence has been described. ${ }^{1-3}$ Clinically apparent anastomotic insufficiency occurs in 5.6-11.9\% of all colorectal resections. ${ }^{4-6}$ As reported in literature, up to $32 \%$ of patients with AL die because of this postoperative complication. ${ }^{4}$ These numbers show the urgent need to prevent AL. Prevention starts with eliminating provoking factors. Decreased blood perfusion at the anastomosis is such a factor that contributes to anastomotic leakage. ${ }^{7-9}$ Therefore, it is hypothesized that a better visualization of the vascularization of the anastomosis will aid in preventing anastomotic leaks. Intraoperatively, the selection of an optimal site for anastomosis with adequate perfusion is now dependent on subjective clinical indicators such as color of the bowel, bleeding of the resection margins, and palpable or visible pulsations of the mesenteric arterial vessels (i.e., the clinical judgement of the surgeon). Karliczek et al. ${ }^{8}$ discussed that there is a lack of reliable intraoperative predictive test for AL by the operating surgeon. Earlier studies used Doppler ultrasound and laser Doppler to assess colorectal anastomotic vascularization. ${ }^{9}$ Peri-anastomotic tissue oxygenation has been evaluated but has not been incorporated in routine practice due to the complexity and limited effectivity of the technique. ${ }^{10}$ There is need for a technique that can accurately and consistently assess bowel perfusion at the anastomotic site in real time. Near-infrared fluorescence (NIRF) imaging allows surgeons to visualize the micro-perfusion of the bowel in real time." This technique, with perioperative indocyanine green (ICG) administration, has been shown feasible in assessing the vascularization of the bowel to be anastomosed. Fluorescence angiography with perioperative ICG administration seems to improve the outcome of laparoscopic anastomotic bowel surgery, in terms of safety and efficiency.11, 12 However, in studies confirming the lower incidence of $A L$, no objective quantification of the signal was performed. ${ }^{1,2,7,13-15}$ Such quantification is desired to make the technique a reliable tool to accurately and consistently assess bowel perfusion at the anastomotic site in real time. C-reactive protein (CRP) is an early predictor of postoperative infection and has been shown to correlate with AL. ${ }^{16-18}$ Intestinal fatty-acid binding protein (I-FABP) is a marker for enterocyte damage. ${ }^{19,} 20$ Earlier research showed a significant higher I-FABP at day 3 postoperatively in patients with AL. ${ }^{21}$ In the same study, calprotectin levels were elevated on day 2 up to day 5 in patients with AL. In this pilot study, we aim to analyze whether a relation exists between the objectively quantified signal of ICG fluorescence angiography during laparoscopic or robotic colorectal surgery and the levels of the serum markers related to anastomotic leakage: CRP, I-FABP, and calprotectin, in order to evaluate the potential of FI to objectively predict AL. To our knowledge, this is the first effort to assess the existence of such a correlation. 


\section{METHODS}

All patients provided written informed consent. This study was approved by the Medical Ethical Committee of Maastricht University Medical Center and conducted according to the Declaration of Helsinki (October 2013, Fortaleza). Included patients were part of the REVEAL study ${ }^{22}$, which is registered at clinicaltrials.gov (NCT02347735).

\section{Study subjects}

Colorectal cancer patients scheduled for either laparoscopic or robotic anastomotic surgery were eligible for inclusion. Patients with inflammatory bowel disease were excluded because this could influence plasma levels of the inflammatory markers. Additional exclusion criteria were known iodine or indocyanine green hypersensitivity, impaired renal function (eGFR <45), pregnancy or breast-feeding (all are contraindications for the use of ICG), and inability to give informed consent. None of the included patients used non-steroidal antiinflammatory drugs (NSAIDs) or corticosteroids. Patient characteristics (sex, age, BMI, medical history, tumor location) and surgical characteristics (type of surgery, anastomotic technique, ostomy, operation time, conversion rate) were collected.

\section{Surgical techniques}

The surgical procedures were performed by experienced laparoscopic colorectal surgeons and started as usual in white light. In laparoscopic cases, a system equipped with fluorescence imaging (Karl Storz GmbH \& CO. KG, Tuttlingen, Germany) with a xenon-based light source (D-light P) was used and in robotic surgeries, the da Vinci ${ }^{\circledR}$ System with Firefly fluorescence imaging (Surgical Intuitive, Sunnyvale, United States of America) was used. Surgery was performed as usual except for three time points, at which a bolus of $0.2 \mathrm{mg} / \mathrm{kg}$ ICG (Verdye; Diagnostic Green Aschheim, Germany) was administered. These three set time points were:

1. Directly after transection of all the vessels supplying the bowel segment to be resected (i.e., after devascularization of the segment)

2. Just before actual transection

3. Directly after the anastomosis is made

After each gift of ICG, the system was switched to NIRF mode and the surgeon was asked to rate the fluorescence intensity of the bowel both proximal and distal to the planned proximal transection line or stapler line on a scale from 1 to 5 . Herein, a score of 1 meant no fluorescent signal, while a score of 5 meant 'maximum fluorescent signal.' Also, the surgeon was asked whether he or she changed the location of transection based on the visualized fluorescent signal. When proximal transection was performed extra-corporally, the operative room was completely darkened in order to capture the fluorescent signal with the respective imaging device. 


\section{Fluorescence intensity measurement}

Postoperatively, the images of the bowel were assessed with OsiriX imaging software (Pixmeo, Geneva, Switzer-land) to quantify the Fl. This software measures the gray value in arbitrary units (AU). At the aforementioned three time points, the $\mathrm{Fl}$ was measured approximately $1 \mathrm{~cm}$ proximal and $1 \mathrm{~cm}$ distal from the proximal transection point or anastomosis and of the background (abdominal wall or fat directly surrounding the relevant bowel part) in a screenshot taken approximately 1 min after ICG administration. In the right hemicolectomies, the distal transection point was used. In case of extracorporeal proximal transection, also three points in the direct surrounding of the bowel were measured as background. The target-to-background ratio (TBR) was defined as the mean Fl in arbitrary units (A.U.) of the bowel wall $1 \mathrm{~cm}$ proximal or distal from the planned proximal (or in right hemicolectomies distal) transection location or $1 \mathrm{~cm}$ from the staple line of the anastomosis minus the Fl of the background divided by the mean Fl in the background (abdominal wall or fat directly surrounding the relevant bowel part).

\section{Definition anastomotic leakage}

The patients were followed up to 30 days after surgery, during which the occurrence of complications and especially $\mathrm{AL}$ was recorded. Clinically relevant $\mathrm{AL}$ was defined and classified according to the definition from the International Study group of Rectal Cancer. ${ }^{23}$ In this definition, $\mathrm{AL}$ is a defect of the anastomotic wall integrity at the colorectal or coloanal anastomotic site leading to a communication between the intra-and extraluminal compartments. A pelvic abscess close to the anastomosis is also considered AL. We applied this definition also to the included colocolic and ileocolic anastomoses in our study. A distinction is made between the severity of AL based on the intervention required:

- Grade A: Anastomotic leakage requiring no active therapeutic intervention

- Grade B: Anastomotic leakage requiring active therapeutic intervention but manageable without relaparotomy;

- Grade C: Anastomotic leakage requiring relaparotomy ${ }^{23}$

\section{Analysis of inflammatory markers}

Venous blood was drawn at hospital admission (preoperative sample) and on day 1, 3, and 5 after surgery, unless the patient was discharged home earlier. C-reactive protein (CRP) plasma levels were determined by using Immunoturbidimetric assay for the in vitro quantitative determination of CRP in human serum and plasma (Roche diagnostics, Germany). Serum calprotectin concentration was determined using a commercially available calprotectin ELISA (Hycult Biotechnology, Uden, The Netherlands). Intestinal fatty-acid binding protein (I-FABP) plasma levels were determined using an in-house ELISA that selectively detects human I-FABP. ${ }^{21}$ 


\section{Statistical analysis}

Basic descriptive statistics were applied to calculate means and standard deviation of the baseline characteristics. Because of the small sample size, non-parametric tests were used to analyze the data retrieved in this study. To compare continuous variables between two groups, the Mann-Whitney test was performed. To compare categorical outcomes between two groups, a Pearson Chi-square crosstab was used. The relation between the measured fluorescence intensity and the values of the inflammatory markers was evaluated using a regression analysis. $\mathrm{P}<0.05$ was considered to denote statistical significance. All statistical analyses were performed with SPSS version 23 (IBM, Armonk, NY, USA).

\section{RESULTS}

\section{Study population}

Thirty patients were included with a mean age of 67.2 (SD 8.2) years of whom 19 (63\%) were male. All under-went elective surgery for colorectal cancer, of which 10 (33.3\%) concerned sigmoid carcinoma, 10 (33.3\%) rec-tum carcinoma, 5 (16.7\%) colon carcinoma in the right hemi-colon, 3 (10\%) cecum carcinoma, and 2 (6.7\%) rectosigmoid carcinoma. Five patients were treated with concomitant chemoradiotherapy before surgery, 4 with only chemotherapy (CAPOX-bevacizumab), and 3 received only radiotherapy $(5 \times 5 \mathrm{~Gy})$. A conversion to open surgery was needed in 7 patients in whom a laparoscopic procedure was started, due to unclear anatomy in obese patients. Further baseline characteristics and surgical characteristics are summarized in Table 1.

\section{Near Infrared fluorescence imaging}

A bolus of $0.2 \mathrm{mg} / \mathrm{kg}$ ICG was administered intravenously. Thus, the total dose ranged between 10.3 and $21.8 \mathrm{mg}$ with a mean of $16.4 \mathrm{mg}$ (SD 3.3). NIRF imaging was aimed to be performed at three time points. In 19 patients, fluorescence imaging was achievable at all three time points. In 10 patients, the first time point was very close to the second time point; therefore, in these patients only just before transection (second time point) and after completion of the anastomosis, the bowel was assessed using NIRF imaging, in order to prevent potential accumulation of the dye at the second time point. In two patients, the anastomosis was too low to visualize the NIRF signal. The time between the first and second time point was on average $68.6 \mathrm{~min}$ (range 9-201) and between the second and third time point on average $40 \mathrm{~min}$ (range 11-84).

\section{Intraoperative decision making based on fluorescence imaging}

In six patients, the dissection location was changed, based on the fluorescent signal. One of these patients developed $\mathrm{AL}$; the remaining 5 patients did not. 
Table 1 Baseline characteristics and surgical characteristics

\begin{tabular}{|c|c|c|}
\hline & $n(\%)$ & Mean (SD) \\
\hline Male & $19(63.3)$ & \\
\hline Age, years & & $67.2(8.2)$ \\
\hline Weight, kg & & $82(16.4)$ \\
\hline $\mathrm{BMI}, \mathrm{kg} / \mathrm{m}^{2}$ & & $27.2(4.9)$ \\
\hline Smoking & $12(40)$ & \\
\hline Both preoperative chemo- and radiotherapy & $5(16.6)$ & \\
\hline Preoperative chemotherapy & $4(13)$ & \\
\hline Preoperative radiotherapy & $3(10)$ & \\
\hline $\begin{array}{l}\text { Location of suspected tumor } \\
\text { Cecum } \\
\text { Right hemi colon } \\
\text { Sigmoid } \\
\text { Recto-sigmoid } \\
\text { Rectum }\end{array}$ & $\begin{array}{l}3(10) \\
5(16.7) \\
10(33.3) \\
2(6.7) \\
10(33.3)\end{array}$ & \\
\hline $\begin{array}{l}\text { Type of surgery } \\
\text { Laparoscopic right hemicolectomy } \\
\text { Laparoscopic sigmoid resection } \\
\text { Laparoscopic low anterior resection } \\
\text { Robot-assisted low anterior resection }\end{array}$ & $\begin{array}{l}8(26.7) \\
11(36.7) \\
4(13.3) \\
7(23.3)\end{array}$ & \\
\hline Deviating stoma & $11(36.7)$ & \\
\hline $\begin{array}{l}\text { Type of anastomosis } \\
\text { Side-to-end } \\
\text { End-to-end } \\
\text { Side-to-side }\end{array}$ & $\begin{array}{l}16(53.3) \\
9(30) \\
5(16.7)\end{array}$ & \\
\hline Conversion to open surgery & $7(23.3)$ & \\
\hline
\end{tabular}

\section{Anastomotic leakage (AL)}

In 5 patients (16\%), AL occurred. In 2 of these cases, only a small abscess around the anastomosis was seen on CT scan and could be treated non-surgically with intravenous administration of antibiotics and CT-guided drainage. These leakages were considered as grade B AL. In 1 patient, AL could be treated with an endo-sponge applied via endoscopy and was also considered as Grade B AL. The remaining 2 patients ( $6 \%$ of the total study population) needed relaparotomy for AL treatment (grade C). All patients with AL underwent a low anterior resection of a rectum or rectosigmoid carcinoma. Two of the AL patients had undergone pre-operative chemoradiotherapy: one preoperative radiotherapy and one preoperative chemotherapy. The remaining patient was not preoperatively treated with neoadjuvant radiotherapy or chemotherapy. There were no statistical differences in age at the time of surgery, preoperative chemotherapy, BMI, administered dose of ICG per bolus (mg), conversion to open surgery, type of anastomosis, TNM classification after surgery, and difficulty of surgery between the patients who developed $A L$ and the patients who did not. There were significantly more smokers in the $A L$ group $(P=0.046)$. The postoperative hospital stay was on average 16 days (median 12 days) in the patients with $A L$, which was 
Table 2 Characteristics of patients with anastomotic leakage compared to those without

\begin{tabular}{|c|c|c|c|}
\hline & No AL (total 25) & AL (total 5) & P-value \\
\hline Male $n$ & $15 / 25$ & $4 / 5$ & 0.397 \\
\hline Age, years (median (IQR)) & $65.5(11.5)$ & $65.0(17)$ & 0.787 \\
\hline $\mathrm{BMI}, \mathrm{kg} / \mathrm{m}^{2}$ (median (IQR)) & $28.3(5.27)$ & $24.4(14.1)$ & 0.627 \\
\hline Smoking $n$ & $8 / 25$ & $4 / 5$ & $0.046^{*}$ \\
\hline Preoperative chemoradiation & $3 / 25$ & $2 / 5$ & 0.183 \\
\hline Preoperative chemotherapy & $3 / 25$ & $1 / 5$ & 0.538 \\
\hline Preoperative radiotherapy & $2 / 25$ & $1 / 5$ & 0.433 \\
\hline Indication for surgery & & & 0.058 \\
\hline Cecum carcinoma & 3 & 0 & \\
\hline Right hemi colon carcinoma & 5 & 0 & \\
\hline Sigmoid carcinoma & 10 & 0 & \\
\hline Rectosigmoid carcinoma & 1 & 1 & \\
\hline Rectum carcinoma & 6 & 4 & \\
\hline Type of surgery & & & 0.107 \\
\hline Laparoscopic right colectomy & 8 & 0 & \\
\hline Laparoscopic sigmoid resection & 10 & 1 & \\
\hline Laparoscopic low anterior resection & 2 & 2 & \\
\hline Robotic low anterior resection & 5 & 2 & \\
\hline Change of dissection place & $5 / 25$ & $1 / 5$ & 1.000 \\
\hline Ileostomy & $7 / 25$ & $4 / 5$ & $0.028^{*}$ \\
\hline Type of anastomosis & & & \\
\hline Side-to-end & 13 & 3 & \\
\hline Side-to-side & 9 & 0 & \\
\hline End-to-end & 3 & 2 & \\
\hline Conversion to open surgery & $6 / 25$ & $1 / 5$ & 0.847 \\
\hline Surgical time, minutes (median (IQR)) & $203.5(95)$ & $287.0(358)$ & 0.344 \\
\hline Postoperative hospital stay, days (median (IQR)) & $4(2.25)$ & $12(22)$ & $0.004^{*}$ \\
\hline
\end{tabular}

significantly longer compared to the patients without this complication, who stayed on average 5 days $(P=0.004)$. A deviating ileostomy had been constructed more often in the AL group. See Table 2 for an overview of these characteristics. Other postoperative complications were postoperative ileus in one patient, which resolved without further consequence. The two patients who needed relaparotomy due to anastomotic leakage both developed pneumonia after the second surgery which was treated with antibiotics.

\section{Subjective fluorescence intensity (FI)}

The FI was scored by the surgeon on a scale from 1 to 5 . In all cases, in all three assessments, this score ranged between 3 and 5 for the bowel proximal to the proximal transection site, or distal transection site in right hemi-colectomies. In the second assessment, the subjective score given for the $\mathrm{Fl}$ was 4 in four of the anastomotic leakage cases. In one of the anastomotic leakage cases, a score of 5 was given, this was one of the cases in which only an abscess close to the anastomosis was present. In the patients who did not develop AL the maximum score of 5 was given in 16 cases $(73 \%)$, which was significantly more often 
Table 3 Subjective fluorescence intensity (FI) scores and anastomotic leakage (AL)

\begin{tabular}{|c|c|c|c|c|c|}
\hline & & FI score 3 & FI score 4 & FI score 5 & $\begin{array}{l}\text { Total no of } \\
\text { patients } \\
\text { assessed }\end{array}$ \\
\hline \multirow[t]{2}{*}{$1^{\text {st }}$ assessment } & No AL & 1 & 5 & 10 & 16 \\
\hline & $\mathrm{AL}$ & 0 & 1 & 3 & 4 \\
\hline \multirow[t]{2}{*}{$2^{\text {nd }}$ assessment } & No AL & 3 & 4 & 18 & 25 \\
\hline & $\mathrm{AL}$ & 0 & 4 & 1 & 5 \\
\hline \multirow[t]{2}{*}{$3^{\text {rd }}$ assessment } & No $A L$ & 2 & 11 & 11 & 24 \\
\hline & $\mathrm{AL}$ & 1 & 1 & 2 & 4 \\
\hline
\end{tabular}

The fluorescence intensity on first and second assessment is scored on the distal side of the distal anastomosis in laparoscopic right hemicolectomies, whilst on the proximal site of the proximal anastomosis in all remaining procedures.

compared to the AL group $(P=0.02)$. In both the first and third assessment, no significant differences in subjective fluorescence intensity between the patients with and without anastomotic leakage were seen. See Table 3 for the given scores.

\section{Measured fluorescence intensity}

Next to subjective scoring by the surgeon, the Fl of the bowel proximal and distal from the proximal transection or distal in right hemicolectomies and the background was measured by image quantification. As shown in Tables 4, 5, 6, and 7, the $\mathrm{FI}$ in patients without $\mathrm{AL}$ was higher at almost all measurements compared to the $\mathrm{Fl}$ in patients who develop $\mathrm{AL}$. This difference is however only statistically significant when we take the background into account and assess the bowel distal from the proximal anastomosis in the 1st assessment ( $P$ $=0.039$ ) (see Table 7).

\section{Inflammatory serum markers}

As shown in Table 8, CRP was significantly higher on postoperative day 5 in anastomotic leakage patients. As is shown in Table 8, all other measured markers do not show a statistical difference between the occurrence of anastomotic leakage and serum levels on day 1, 3, or 5 .

\section{Fluorescence intensity in relation to inflammatory serum markers}

The relation between the respective serum markers CRP, Calprotectin and I-FABP, and the fluorescent signal was assessed using a regression analysis. For none of the serum markers on any time point, a significant relation could be found with the fluorescence both proximal and distal or measured as TBR, with one exception. At the third time point, after the anastomosis is made, a higher TBR of the distal part of the anastomosis was related to a lower serum calprotectin level on postoperative day $5(P=0.046)$. 
Table 4 Fluorescence intensity (FI) bowel proximal from the proximal transection line and anastomotic leakage $(\mathrm{AL})$ (distal from distal transection line in right hemicolectomies, thus side from the transection line that stays in the patient).

\begin{tabular}{llll}
\hline & Median FI no AL & Median FI AL & P-value \\
\hline $1^{\text {st }}$ assessment & $38.8(61.2)$ & $34.2(28.7)$ & 0.494 \\
\hdashline $2^{\text {nd }}$ assessment & $49.2(45.0)$ & $44.1(94.6)$ & 0.385 \\
\hline $3^{\text {rd }}$ assessment & $86.5(54.8)$ & $99.5(68.1)$ & 0.336 \\
\hline
\end{tabular}

Median fluorescence intensity (interquartile range)

Table 5 Fluorescence intensity (FI) bowel distal from the proximal transection-line and anastomotic leakage $(\mathrm{AL})$ (proximal from the distal transection line in right hemicolectomies, thus side from the transection line that will be resected)

\begin{tabular}{lccc}
\hline & Median FI no AL & Median FI AL & P-value \\
\hline $1^{\text {st }}$ assessment & $31.7(23.7)$ & $36.7(27.1)$ & 0.064 \\
\hline $2^{\text {nd }}$ assessment & $40.8(32.4)$ & $64.5(72.8)$ & 0.448 \\
\hline $3^{\text {rd }}$ assessment & $59.9(37.6)$ & $55.9(30.5)$ & 0.952 \\
\hline
\end{tabular}

Median fluorescence intensity (interquartile range)

Table 6 Target to background ratio (TBR) bowel proximal from the proximal transection line and anastomotic leakage $(A L)$ (distal from distal transection line in right hemicolectomies, thus side from the transection line that stays in the patient)

\begin{tabular}{llcc}
\hline & Median TBR no AL & Median TBR AL & P-value \\
\hline $1^{\text {st }}$ assessment & $2.8(4.7)$ & $1.1(1.4)$ & 0.820 \\
\hdashline $2^{\text {nd }}$ assessment & $2.2(13)$ & $0.5(2.1)$ & 0.229 \\
\hdashline $3^{\text {rd }}$ assessment & $1.4(2.6)$ & $1.4(1.0)$ & 0.669 \\
\hline
\end{tabular}

Median fluorescence intensity (Interquartile range)

Table 7 Target to background ratio (TBR) bowel distal from the proximal transection line and anastomotic leakage $(A L)$ (proximal from distal transection line in right hemicolectomies, thus the side from the transection line that will be resected)

\begin{tabular}{llcc}
\hline & Median TBR no AL & Median TBR AL & P-value \\
\hline $1^{\text {st }}$ assessment & $3.4(6.9)$ & $1.2(1.3)$ & 0.039 \\
\hline $2^{\text {nd }}$ assessment & $2.6(13.5)$ & $1.8(4.0)$ & 0.482 \\
\hdashline $3^{\text {rd }}$ assessment & $1.4(2.9)$ & $0.9(0.1)$ & 0.364 \\
\hline
\end{tabular}

Median fluorescence intensity (interquartile range)

\section{DISCUSSION}

NIRF angiography with ICG is used more and more in colorectal surgery as this technique holds the potential to lower the risk for anastomotic leakage. ${ }^{11}$ However, it is unknown when the bowel is fluorescent enough to actually predict optimal anastomotic healing. In this respect, a cut-off value is desirable. To come to such consensus, we first need to know 
Table 8 Serum markers anastomotic leakage versus no anastomotic leakage.

\begin{tabular}{|c|c|c|c|}
\hline & $\begin{array}{l}\text { No anastomotic } \\
\text { leakage }\end{array}$ & $\begin{array}{l}\text { Anastomotic } \\
\text { leakage }\end{array}$ & p-value \\
\hline \multicolumn{4}{|l|}{ Mean CRP } \\
\hline Pre-operative $(\mathrm{mg} / \mathrm{ml}) \pm \mathrm{SD}$ & $2.9 \pm 2.3$ & $2.1 \pm 0.9$ & 0.69 \\
\hline Postoperative day $1(\mathrm{mg} / \mathrm{ml}) \pm \mathrm{SD}$ & $87.4 \pm 35.8$ & $87.4 \pm 35.3$ & 0.90 \\
\hline Postoperative day $3(\mathrm{mg} / \mathrm{ml}) \pm \mathrm{SD}$ & $101.1 \pm 48.0$ & $183.6 \pm 123.5$ & 0.09 \\
\hline Postoperative day $5(\mathrm{mg} / \mathrm{ml}) \pm S D$ & $48.5 \pm 24.66$ & $152.8 \pm 90.7$ & $0.01 *$ \\
\hline \multicolumn{4}{|l|}{ Mean Calprotectin } \\
\hline Preoperative (ng/ml (SD) & $822.9 \pm 765.4$ & $3451.6 \pm 3987.1$ & 0.41 \\
\hline Postoperative day $1(\mathrm{ng} / \mathrm{ml}) \pm \mathrm{SD}$ & $7587.3 \pm 17589.9$ & $7743.5 \pm 12026.8$ & 1.0 \\
\hline Postoperative day $3(\mathrm{ng} / \mathrm{ml}) \pm \mathrm{SD}$ & $2467.0 \pm 1430.3$ & $1683.3 \pm 1549.4$ & 0.15 \\
\hline Postoperative day $5(\mathrm{ng} / \mathrm{ml}) \pm S D$ & $3326.7 \pm 2783.0$ & $2756.1 \pm 1974.3$ & 0.68 \\
\hline \multicolumn{4}{|l|}{ I-FABP } \\
\hline Preoperative $(\mathrm{pg} / \mathrm{ml}) \pm \mathrm{SD}$ & $805 \pm 376.6$ & $520.1 \pm 516.1$ & 0.16 \\
\hline Postoperative day $1(\mathrm{pg} / \mathrm{ml}) \pm \mathrm{SD}$ & $644.7 \pm 370.4$ & $383.2 \pm 160.4$ & 0.14 \\
\hline Postoperative day $3(\mathrm{pg} / \mathrm{ml}) \pm S D$ & $574.8 \pm 431.4$ & $373.9 \pm 335.3$ & 0.31 \\
\hline Postoperative day $5(\mathrm{pg} / \mathrm{ml}) \pm S D$ & $496.9 \pm 291.4$ & $143.0 \pm 49.6$ & 0.05 \\
\hline
\end{tabular}

whether a "more fluorescent bowel" actually is indicative for a lower risk for anastomotic leakage and accompanying lower postoperative inflammatory parameters. Therefore, we performed this clinical pilot study, in which we aimed to correlate the signal strength of fluorescence angiography with the occurrence of $A L$ and with the level of the serum markers that are thought to be predictive of AL: CRP, calprotectin, and I-FABP. The fluorescence intensity was measured after administration of $0.02 \mathrm{mg} / \mathrm{kg}$ ICG intravenously at three time points. CRP, calprotectin and I-FABP were determined preoperatively and on day 1, 3, and 5 after surgery. Follow-up for anastomotic leakage was until 30 days postoperatively.

In the current study, 5 (16.7\%) patients developed AL. Three of these leakages were considered a grade $\mathrm{B}$ leakage, while the remaining two were grade $\mathrm{C}$ anastomotic leakages which needed re-laparoscopy. The number of patients with AL is somewhat higher compared to the literature (5.6-11.9\% $)^{4-6}$, and especially when compared to other studies in which NIRF angiography is used during colorectal surgery. ${ }^{11}$ This could be explained by the fact that both grade $B$ and grade $C$ anastomotic leakage were taken into account in this study, while other studies may have only considered grade C leakage only. In addition, 40\% of patients smoked, which is a risk factor for developing AL.4, 24 Remarkably, preoperative radiotherapy, which is also a known risk for leakage was not identified as such in our study. The limited number of patients may have contributed to this discrepancy.

In all cases, the subjective score for $\mathrm{Fl}$ proximal from the (planned) proximal transection line or distal from distal transection line (in right hemicolectomies), ranged between 3 and 5 on a scale from 1 to 5 . For the bowel proximal from the transection line, the maximum score of 5 was given significantly more often in the patients that healed without a leak. This is in line with the study by Ris et al. ${ }^{2}$ in which the fluorescence score ranges from 'good,' 'average,' to 'bad.' In all patients, the fluorescence was scored 'good' by the surgeon and 
none of the patients developed AL. Other studies scored the fluorescence intensity as 'good enough' or 'not good enough' based on subjective score of the surgeon. In these studies, if the fluorescence intensity was considered not good enough, the dissection place was changed. ${ }^{13,25-29}$ The anastomotic leakage rate in patients with changed dissection level was equal ${ }^{13,25-27,29}$ or higher ${ }^{28}$ compared to the patients in which no change was needed after NIRF imaging. A possible explanation for a higher leak rate after change of transection location may be the fact that a clearly insufficiently perfused bowel end may be indicator of a longer segment of moderate perfusion. This emphasizes the need for cut-off values for level of perfusion. ${ }^{11}$ In the current study, revision of surgical plan based on the FI of the bowel occurred in six patients. One of these developed AL. These numbers are too low to draw conclusions.

In the present study, the objectively measured Fl was higher in the patients who did not develop AL. This difference was only significant when taking the background into account and expressing data as TBR. By using TBR, a correction is made for the distance between the endoscope and the bowel. A shorter distance between the laparoscope and the target causes a higher $\mathrm{Fl}^{30}$ By taking the background into account, this effect is minimized, since the background will also show increased fluorescence intensity when holding the laparoscope closer to the tissue. Such correction of the signal seems necessary, as in practice, it is not always possible to exactly measure and standardize the distance to the bowel.

According to literature, the CRP trajectory is highly predictive for AL. ${ }^{6}$ In the current series, CRP on postoperative day 5 was significantly higher in patient who developed AL. This observation is in line with findings reported in literature. An earlier study by Reisinger et al. ${ }^{21}$ showed a rise in postoperative CRP in patients with an anastomotic leak, which was only significant on day 4. Bigin et al. ${ }^{31}$ showed a significant higher CRP on postoperative day 3 , but unfortunately CRP was not measured on postoperative day 5 . Reynolds et al. ${ }^{17}$ found a significantly higher CRP level in patients with grade C AL on day 5 up to day 7. Unfortunately, in the current study, no relation of CRP and subjective or measured fluorescence intensity or TBR could be found.

Calprotectin is a non-specific marker for acute inflammation ${ }^{32}$. An earlier study by Reisinger et al. ${ }^{21}$ showed higher calprotectin levels on postoperative day 2, 3, 4, and 5 in patients who developed AL. Similar results were found by Cikot et al. ${ }^{33}$ In the current study however, no statistical difference in serum calprotectin levels could be found between patients with and without anastomotic leakage on postoperative day 1, 3, or 5. Interestingly, serum calprotectin levels on postoperative day 5 were negatively correlated with TBR of the distal part of the anastomosis. This relation needs to be confirmed in a larger study to determine its clinical relevance.

I-FABP is an intestinal cell damage marker. ${ }^{21}$ An earlier study by Reisinger et al. ${ }^{21}$ showed significantly higher I-FABP levels preoperatively in patients who developed anastomotic leakage. The I-FABP levels remained higher in these patients after surgery, but only 
statistically significant on postoperative day 3. In the current study however, I-FABP levels were lower at all time points in the patients who developed anastomotic leakage, although this difference was not significant. Moreover, we could not detect a significant relation between $\mathrm{FI}$ and serum I-FABP values in this study.

A possible explanation for this discrepancy might be the low specificity of I-FABP. This marker for enterocyte dam-age is not only elevated in case of inflammation, but also in patients with colorectal cancer compared to patients with-out colorectal cancer. ${ }^{34}$ When only taking the patients with a grade $\mathrm{C}$ anastomotic leakage into account, a (non-statistically significant) higher I-FABP value is found in the leakage patients. More research is needed to determine its place in the management of the colorectal operative patient.

A higher fluorescence intensity was found in all cases in the bowel at the side that will remain in the patients after surgery. This means that the de-vascularized bowel results in a lower signal, indicating that the fluorescent signal does reflect bowel perfusion.

This study has some limitations. First, it was designed as a pilot study to explore whether there is a potential relation between $\mathrm{Fl}$ and inflammatory serum markers that correspond with AL. A relatively small and heterogenous group of patients was included. With the markers and the patient group chosen here, it was not yet possible to identify a cut-off value of fluorescent signal that indicates an optimal anastomotic healing potential. A more homogenous patient group (e.g., only right hemicolectomies or rectal resections) might have given different results. Another factor of influence could be that only the measurements at single time points were performed, and not an evaluation of the signal intensity over time after administration of the respective boluses of ICG. The small number of included patients can be an explanation for finding few statistical significant differences for most outcome parameters.

In this study, several time points of ICG administration were chosen. Although arterially the dye washes out very quickly - which is evident when observed with the naked eye-it cannot be ruled out that some retention of the dye occurred. Although this phenomenon will take place in both the bowel wall and the background, this may have influenced the objective measurements. The time between the first and second dose administration was on average $68.6 \mathrm{~min}$ (range 9-201) and between the second and third bolus of ICG $40 \mathrm{~min}$ (range 11-84). A cut-off value for the optimal interval is not known in literature.

In conclusion, both subjective and measured FI seem to be related to the occurrence of $\mathrm{AL}$. In this pilot study, no relation between $\mathrm{FI}$ and inflammatory serum markers could yet be found nor a cut-off value of Fl predicting AL. A larger study based on the current study, with a single time point of measurement in a homogenous population seems preferable to enhance the chance that an objective cut-off value of the fluorescent signal to predict AL will be identified. Ideally, future research identifies such cut-off value, but also leads to presenting the objective result of fluorescence measurement real time to the surgeon, thereby supplying the surgeon with essential per-operative information for the optimal transection point to prevent anastomotic leakage. 


\section{REFERENCES}

1. Sherwinter DA, Gallagher J, Donkar T (2013) Intra-operative transanal near infrared imaging of colorectal anastomotic perfu-sion: a feasibility study. Colorectal Dis 15(1):91-96

2. Ris F, Hompes R, Cunningham C, Lindsey I, Guy R, Jones O et al (2014) Near-infrared (NIR) perfusion angiography in minimally invasive colorectal surgery. Surg Endosc 28(7):2221-2226

3. Ha GW, Kim JH, Lee MR (2017) Oncologic impact of anasto-motic leakage following colorectal cancer surgery: a systematic review and meta-analysis. Ann Surg Oncol 24(11):3289-3299

4. Park JS, Choi GS, Kim SH, Kim HR, Kim NK, Lee KY et al (2013) Multicenter analysis of risk factors for anastomotic leakage after laparoscopic rectal cancer excision: the Korean laparoscopic colorectal surgery study group. Ann Surg 257(4):665-671

5. Zhang W, Lou Z, Liu Q, Meng R, Gong H, Hao L et al (2017) Multicenter analysis of risk factors for anastomotic leakage after middle and low rectal cancer resection without diverting stoma: a retrospective study of 319 consecutive patients. Int J Colorectal Dis 32(10):1431-143

6. Smith SR, Pockney P, Holmes R, Doig F, Attia J, Holliday E et al (2017) Biomarkers and anastomotic leakage in colorectal surgery: C-reactive protein trajectory is the gold standard. ANZ J Surg 88(5):440-444

7. Jafari MD, Lee KH, Halabi WJ, Mills SD, Carmichael JC, Stamos MJ et al (2013) The use of indocyanine green fluorescence to assess anastomotic perfusion during robotic assisted laparoscopic rectal surgery. Surg Endosc 27(8):3003-3008

8. Karliczek A, Harlaar NJ, Zeebregts CJ, Wiggers T, Baas PC, van Dam GM (2009) Surgeons lack predictive accuracy for anasto-motic leakage in gastrointestinal surgery. Int J Colorectal Dis 24(5):569-576

9. Vignali A, Gianotti L, Braga M, Radaelli G, Malvezzi L, Di Carlo V (2000) Altered microperfusion at the rectal stump is predictive for rectal anastomotic leak. Dis Colon Rectum 43(1):76-82

10. Nachiappan S, Askari A, Currie A, Kennedy RH, Faiz O (2014) Intraoperative assessment of colorectal anastomotic integrity: a systematic review. Surg Endosc 28(9):2513-2530

11. van den Bos J, Al-Taher M, Schols RM, van Kuijk S, Bouvy ND, Stassen LPS (2018) Near-infrared fluorescence imaging for real-time intraoperative guidance in anastomotic colorectal surgery: a systematic review of literature. J Laparoendosc Adv Surg Tech A 28(2):157-167

12. Blanco-Colino R, Espin-Basany E. Intraoperative use of ICG fluorescence imaging to reduce the risk of anastomotic leakage in colorectal surgery: a systematic review and meta-analysis. Tech Coloproctol. 2017

13. Boni L, David G, Mangano A, Dionigi G, Rausei S, Spampatti S et al (2015) Clinical applications of indocyanine green (ICG) enhanced fluorescence in laparoscopic surgery. Surg Endosc 29(7):2046-2055

14. Kudszus S, Roesel C, Schachtrupp A, Hoer JJ (2010) Intraop-erative laser fluorescence angiography in colorectal surgery: a noninvasive analysis to reduce the rate of anastomotic leakage. Langenbeck's archives of surgery. Deutsche Gesellschaft fur Chi-rurgie 395(8):10251030

15. Diana M, Agnus V, Halvax P, Liu YY, Dallemagne B, Schlagowski Al et al (2015) Intraoperative fluorescence-based enhanced reality laparoscopic real-time imaging to assess bowel perfusion at the anastomotic site in an experimental model. Br J Surg 102(2):e169-e176

16. Facy O, Paquette B, Orry D, Santucci N, Rat P, Rat P et al (2017) Inflammatory markers as early predictors of infection after colo-rectal surgery: the same cut-off values in laparoscopy and lapa-rotomy? Int J Colorectal Dis 32(6):857-863

17. Reynolds IS, Boland MR, Reilly F, Deasy A, Majeed MH, Deasy J et al (2017) C-reactive protein as a predictor of anastomotic leak in the first week after anterior resection for rectal cancer. Colorectal Dis 19(9):812-818

18. Munoz JL, Alvarez MO, Cuquerella V, Miranda E, Pico C, Flo-res R et al (2018) Procalcitonin and 
C-reactive protein as early markers of anastomotic leak after laparoscopic colorectal surgery within an enhanced recovery after surgery (ERAS) program. Surg Endosc 32(9):4003-4010

19. Relja B, Szermutzky M, Henrich D, Maier M, de Haan JJ, Lubbers T et al (2010) Intestinal-FABP and liver-FABP: Novel markers for severe abdominal injury. Acad Emerg Med 17(7):729-735

20. Schellekens DH, Grootjans J, Dello SA, van Bijnen AA, van Dam RM, Dejong CH et al (2014) Plasma intestinal fatty acid-binding protein levels correlate with morphologic epithelial intestinal damage in a human translational ischemia-reperfusion model. J Clin Gastroenterol 48(3):253-260

21. Reisinger KW, Poeze M, Hulsewe KW, van Acker BA, van Bijnen AA, Hoofwijk AG et al (2014) Accurate prediction of anastomotic leakage after colorectal surgery using plasma markers for intesti-nal damage and inflammation. J Am Coll Surg 219(4):744-751

22. Jongen AC, Bosmans JW, Kartal S, Lubbers T, Sosef M, Slooter GD et al (2016) Predictive factors for anastomotic leakage after colorectal surgery: study protocol for a prospective observational study (REVEAL Study). JMIR Res Protoc 5(2):e90

23. Rahbari NN, Weitz J, Hohenberger W, Heald RJ, Moran B, Ulrich A et al (2010) Definition and grading of anastomotic leakage fol-lowing anterior resection of the rectum: a proposal by the Inter-national Study Group of Rectal Cancer. Surgery 147(3):339-351

24. Kawada K, Hasegawa S, Hida K, Hirai K, Okoshi K, Nomura A et al (2014) Risk factors for anastomotic leakage after laparo-scopic low anterior resection with DST anastomosis. Surg Endosc 28(10):2988-2995

25. Boni L, Fingerhut A, Marzorati A, Rausei S, Dionigi G, Cassi-notti E (2017) Indocyanine green fluorescence angiography dur-ing laparoscopic low anterior resection: results of a casematched study. Surg Endosc 31(4):1836-1840

26. Boni L, David G, Dionigi G, Rausei S, Cassinotti E, Fingerhut A (2016) Indocyanine greenenhanced fluorescence to assess bowel perfusion during laparoscopic colorectal resection. Surg Endosc 30(7):2736-2742

27. Kin C, Vo H, Welton L, Welton M (2015) Equivocal effect of intraoperative fluorescence angiography on colorectal anastomotic leaks. Dis Colon Rectum 58(6):582-587

28. Hellan M, Spinoglio G, Pigazzi A, Lagares-Garcia JA (2014) The influence of fluorescence imaging on the location of bowel tran-section during robotic left-sided colorectal surgery. Surg Endosc 28(5):1695-1702

29. Jafari MD, Wexner SD, Martz JE, McLemore EC, Margolin DA, Sherwinter DA et al (2015) Perfusion assessment in laparoscopic left-sided/anterior resection (PILLAR II): a multi-institutional study. J Am Coll Surg 220(1):82-92 e1

30. van den Bos J, Wieringa FP, Bouvy ND, Stassen LPS (2018) Optimizing the image of fluorescence cholangiography using ICG: a systematic review and ex vivo experiments. Surg EndosC 32(12):4820-4832

31. Bilgin IA, Hatipoglu E, Aghayeva A, Arikan AE, Incir S, Mamal Torun M et al (2017) Predicting value of serum procalcitonin, c-reactive protein, drain fluid culture, drain fluid interleukin-6, and tumor necrosis factor-alpha levels in anastomotic leakage after rectal resection. Surg Infect 18(3):350-356

32. Johne B, Fagerhol MK, Lyberg T, Prydz H, Brandtzaeg P, Naess-Andresen CF et al (1997) Functional and clinical aspects of the myelomonocyte protein calprotectin. Mol Pathol 50(3):113-123

33. Cikot M, Kones O, Gedikbasi A, Kocatas A, Karabulut M, Temizgonul KB et al (2016) The marker C-reactive protein is helpful in monitoring the integrity of anastomosis: plasma calprotectin. Am J Surg 212(1):53-61

34. Bingold TM, Franck K, Holzer K, Zacharowski K, Bechstein WO, Wissing H et al (2015) Intestinal fatty acid binding protein: a sensitive marker in abdominal surgery and abdominal infection. Surg Infect 16(3):247-253 


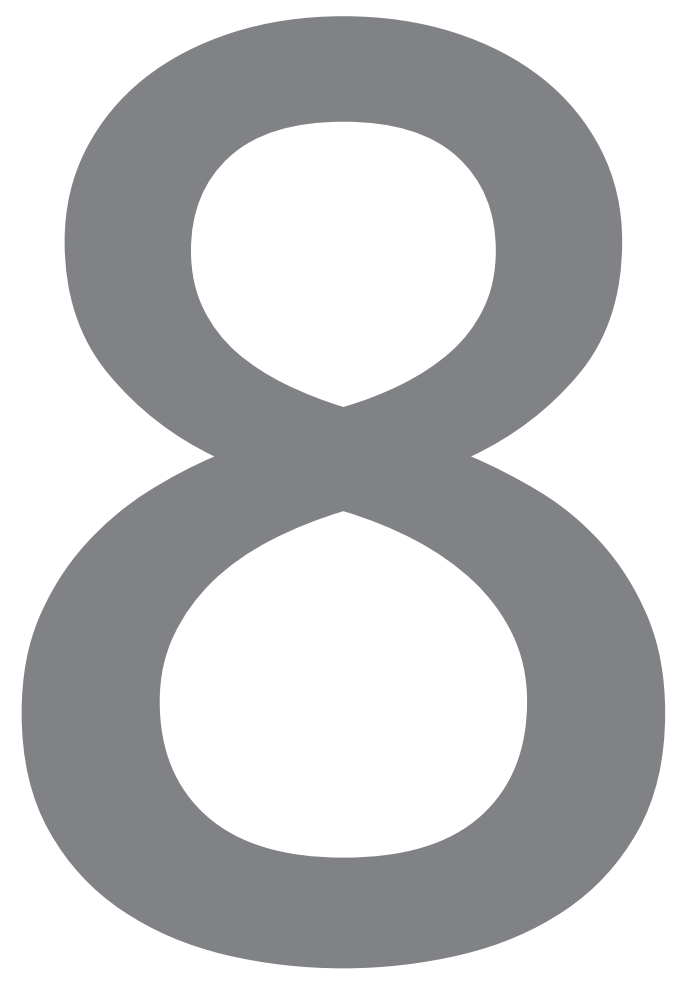




\section{Fluorescence ureteral visualization in human laparoscopic colorectal surgery using methylene blue}

Mahdi Al-Taher, Jacqueline van den Bos, Rutger M. Schols, Nicole D. Bouvy, Laurents P.S. Stassen.

Journal of laparoendoscopic and advanced surgical techniques 2016;26(11):870-75 


\section{ABSTRACT}

\section{Background}

Ureteral injury during laparoscopic surgery is rare, but when it occurs, it can be a serious problem. Near-infrared fluorescence (NIRF) with methylene blue (MB) administration is a promising technique for easier and potentially earlier intraoperative visualization of the ureter. Aim of this prospective study was to assess the feasibility of NIRF imaging of the ureter during laparoscopic colorectal surgery, using MB.

\section{Methods}

Patients undergoing laparoscopic colorectal surgery were included and received intravenous injection of MB preoperatively. The ureter was visualized using a laparoscope, which offered both conventional and fluorescence imaging. Intraoperative recognition of the ureter was registered. The precision of ureter distinction with MB imaging was compared to the conventional laparoscopic view.

\section{Results}

Ten patients were included. All procedures were initially performed using a laparoscopic approach. Dose per injection ranged between $0.125 \mathrm{mg} / \mathrm{kg}$ and $1.0 \mathrm{mg} / \mathrm{kg}$ bodyweight. There were no adverse effects attributable to MB administration. The ureter was successfully detected in five patients, with highest contrast between ureter and surrounding tissue at an administered dose of $0.75-1.0 \mathrm{mg} / \mathrm{kg}$. The fluorescent signal was only picked up after the ureter was already visible in the conventional white light mode.

\section{Conclusion}

Ureteral fluorescence imaging using MB proved to be safe and feasible. However, the present technique does not provide practical advantage over conventional laparoscopic imaging for identification of the ureter during laparoscopic colorectal surgery. Future research is necessary to explore more extensive dose finding, alternative fluorescent dyes, or improvement of the imaging system to make this application clinically beneficial. 


\section{INTRODUCTION}

There is great public attention on the safety of surgery, especially in laparoscopic surgery. In addition, efficiency is of constant concern in modern healthcare. Laparoscopic colorectal surgery is an advanced procedure requiring a high level of endoscopic skills. Finding the right tissue structures and anatomical planes takes time and incorporates risks. latrogenic ureteral, vascular, or nerve injury are complications that may occur.

Ureteral injury during surgery is rare, but when it occurs, constitutes a serious problem. The major causes are trauma, iatrogenic injury during open surgery, and injury during catheter or endoscopic intervention. Ureteral injuries are often insidious since they are found only after other, more profound injuries are addressed, which may lead to worse outcomes. Risk factors for iatrogenic injury during colorectal surgery are previous pelvic operations, infection, and inflammatory bowel disease, but most ureteral injuries occur in patients lacking these risk factors. ${ }^{2}$ Earlier studies have reported an incidence of $0.1 \%$ to $7.6 \%$ in colorectal and gynecologic surgery, with more than $80 \%$ of cases going unrecognized intraoperatively. ${ }^{3-6}$ Assimos et al. ${ }^{6}$ compared the incidence of iatrogenic ureteral injuries between the pre-laparoscopic and laparoscopic era and found that the incidence of iatrogenic ureteral injuries was significantly greater in the latter. Thus, the early detection and prevention of ureteral injury are important. If in any other way than with normal vision and common anatomical knowledge, essential anatomical planes and structures could be identified, this would beneficially influence the safety and efficiency of laparoscopic colorectal surgery.

To help avoid injury, intravenous pyelography, retrograde pyelography, or urologic computed tomography can be performed preoperatively; however, none of these imaging techniques provides real-time guidance during the actual procedure. ${ }^{7}$ Another applied method is ureteral stent placement. However, in laparoscopic surgery, the results are not convincing and apart from added operation time, can cause complications itself. $2,8,9$

Intraoperative fluorescence ureteral identification with preoperative optical dye administration is a promising new technique for easier and earlier intraoperative visualization of the ureter and could thereby improve the outcome -safety and efficiencyof laparoscopic colorectal surgery. ${ }^{10,11}$

Matsui et al. ${ }^{10}$ showed that the clinically available dye methylene blue (MB) has near-infrared (NIR) fluorescence properties (even in very low doses) that permit realtime intraoperative visualization of the ureters in a pig model in open and laparoscopic procedures. Verbeek et al..$^{12}$ were able to visualize the ureters during open human surgery, using MB in combination with a near-infrared fluorescence (NIRF) imaging system.

We hypothesize that the application of the NIRF technique using MB during laparoscopic colorectal surgery can improve the visualization of the ureter, thereby speeding up the time of dissection and identification of the ureter, which increases patient safety by lowering the risk of ureteral injury. Therefore, in this study, we assessed the feasibility of this technique for the detection of ureters in patients undergoing elective laparoscopic colorectal surgery. 


\section{MATERIALS AND METHODS}

\section{Patients}

This prospective observational study has been approved by the Medical Ethics Committee of the Maastricht University Medical Center and was performed in accordance with the ethical standards of the Helsinki Declaration of 1975. The study was conducted in the Maastricht University Medical Center (MUMC) and registered in the Netherlands National Trial Register: registration number NTR3605 (www.trialregister.nl/trialreg/adminrctview.asp?TC=3605). Randomly chosen patients meeting the inclusion criteria (Table 1) were enrolled between January 2014 and July 2015. All patients scheduled to undergo laparoscopic colorectal surgery, in which identification of the left ureter was part of the standard procedure, were eligible for participation in the study. All included patients provided written informed consent and the collected data were anonymized.

\section{Intraoperative imaging system}

A commercially available laparoscopic fluorescence imaging system (Karl Storz GmbH \& CO. KG, Tuttlingen, Germany) was used. This system has been described in more detail previously.13 For this study, the system had been adapted with a special filter to detect the fluorescent signal of MB, which is at around $670 \mathrm{~nm}$. The equipment included a plasma light guide and a 30-degree 10-mm laparoscope applicable for NIRF imaging. Procedures were digitally recorded.

\section{MB preparation and injection}

Methylthionine chloride (Methylene blue [MB]) $10 \mathrm{mg} / \mathrm{mL}$ was prepared by the hospital pharmacy of the Catharina Hospital (Eindhoven, the Netherlands). After intravenous administration, $\mathrm{MB}$ is rapidly taken up by the tissues. The majority of the dose is excreted in the urine, usually in the form of leucomethylthioninium chloride. Peak MB absorbance and emission occur at 668 and $688 \mathrm{~nm}$, respectively. ${ }^{10} \mathrm{An}$ intravenous MB solution for injection was administered preoperatively. Fluorescence imaging was performed at various moments during the phase of surgical dissection in which ureteral identification was relevant.

\section{Surgical technique}

The operations were performed by gastrointestinal surgeons with extensive experience in laparoscopic colorectal surgery. MB was administered using a preoperative intravenous injection in a vein of the upper extremity. Doses ranged from $0.25 \mathrm{mg} / \mathrm{kg}$ up to $1.0 \mathrm{mg} /$ $\mathrm{kg}$ body weight. In the course of the study, these were increased based on previous publications..$^{10,14}$ The dye administration occurred during the induction of anesthesia and ranged between 40 and 0 minutes before the introduction of the first trocar. 
Table 1 Inclusion and exclusion criteria for participation in the study

\begin{tabular}{ll}
\hline Inclusion criteria & Exclusion criteria \\
\hline $\begin{array}{l}\text { Patients scheduled for laparoscopic colorectal } \\
\text { surgery (i.e., rectal surgery, sigmoid resection) }\end{array}$ & Not able to give written informed consent \\
\hline No hypersensitivity for methylene blue & Known methylene blue hypersensitivity \\
\hline Males and females (not pregnant) & Pregnant, breast-feeding women \\
\hline Age $>18$ years & Age $<18$ years \\
\hline $\begin{array}{l}\text { Able to understand the nature of the study and } \\
\text { what will be required }\end{array}$ & Medication use: SSRI's \\
\hline \begin{tabular}{l} 
Willing to participate \\
\hline
\end{tabular} & Not willing to participate \\
\hline
\end{tabular}

After surgery, the NIRF technique was assessed using an intraoperative registration form and visual recordings. Visual (both conventional and fluorescence) recordings were obtained during the same laparoscopic procedure. Urine from four included patients was collected from the bladder catheter at the end of the surgical procedure for NIRF imaging.

\section{Ex vivo assessment of the NIRF technique}

As a quality check of the procedure, in an ex vivo experiment, urine samples of two healthy volunteers were collected and accumulating concentrations of MB diluted in urine were made. Thereafter, fluorescence imaging was performed in a dark room with the camera at a $2 \mathrm{~cm}$ proximity of the sample. For each sample, a target to background ratio (TBR) was calculated as explained below. For this calculation, a vial filled with sterile phosphatebuffered saline was used as the background.

\section{Target-to-background ratio}

In image-guided surgery research, the target-to-background ratio is commonly used for analyzing recordings. For assessment of the degree of fluorescent illumination, OsiriX 5.0.1 Imaging Software was used. The fluorescent images were analyzed by determining the TBR. The fluorescence intensity of the target was measured as a mean of three point regions of interest in the ureter minus the mean fluorescence intensity of three regions in the immediate surrounding tissue as background, divided by the mean fluorescence intensity of the background. Hence, the following formula was used: TBR = (Fluorescence intensity of Target - Fluorescence intensity of Background)/Fluorescence intensity of Background.

\section{RESULTS}

A total of ten patients were included in this study. Patient characteristics and clinical data are summarized in Table 2. Median age was 73.4 years (range 52-87) and median BMI was 27.44 $\mathrm{kg} / \mathrm{m}^{2}$ (range 23.24-37.04). All patients were scheduled to undergo elective laparoscopic 

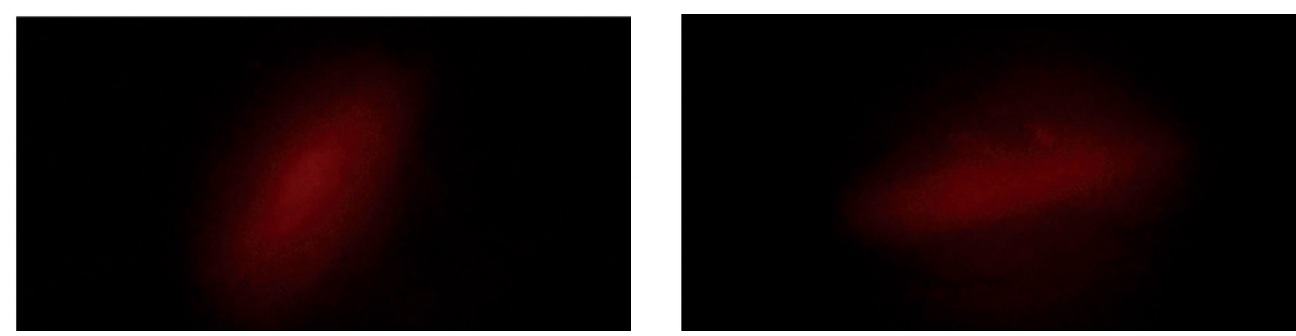

Figure 1 Screenshot of ureter visualization with fluorescence imaging at dosing of Methylene Blue of $1.0 \mathrm{mg} /$ $\mathrm{kg}$ (top screenshot) and $0.75 \mathrm{mg} / \mathrm{kg}$ (bottom screenshot). In this screenshot, red is the illumination of the ureter and black is surrounding tissue

surgery for colorectal cancer. Administration of the dye ranged from 0 to 40 minutes before the introduction of the first trocar.

A dose of 0.125 up to $1.0 \mathrm{mg} / \mathrm{kg}$ resulting in a total dose of $8-90 \mathrm{mg}$ per patient was administered preoperatively. The dose was gradually increased during the time span of the study, due to disappointing results of the low doses. In only two of the six patients in which a concentration of $0.125-0.25 \mathrm{mg} / \mathrm{kg} \mathrm{MB}$ was administered, there was (weak) visualization of the ureter in the fluorescence mode. In the other four, up to 100 minutes after administration, no fluorescent imaging of the ureter could be obtained, even after the detection of the ureter in white light mode. One of these procedures was converted to open procedure (due to intra-abdominal adhesions) before it was possible to analyze the ureter in fluorescence mode.

In one of two patients with a dosing of $0.5 \mathrm{mg} / \mathrm{kg}$, the ureter was detected in fluorescence mode. This occurred with a weak signal and after the ureter had already been identified in white light mode. In the other patient, conversion to an open procedure was performed (because of intra-abdominal adhesions) before actual imaging of the ureter in fluorescence mode could take place.

In the remaining two patients, a concentration of 0.75 and $1 \mathrm{mg} / \mathrm{kg}$ showed a clear delineation of the ureter during the pulsatile movement of the urine through the ureter (see example screenshot in Fig. 1). The laparoscope had to be held close (within a few centimeters) to the ureter to obtain this visualization.

In four consecutive patients (patients number 5-8 in Table 2), urine was collected from the bladder catheter at the end of the procedure. Ex vivo fluorescence imaging with the same camera system as explained in the methods section showed a clear fluorescence signal of all four urine samples with a TBR varying between 6.88 and 9.2 (Table 3).

In the ex vivo experiment, a strong fluorescence signal in a wide range of considerable dilutions was obtained (Table 4).

Very high concentrations of $M B$ revealed no signal $(T B R<0)$ and in very high dilutions, only a weak, although still present, signal. 
Table 2 Patient characteristics and ureteral identification results

\begin{tabular}{|c|c|c|c|c|c|c|c|}
\hline No. & Sex & $\begin{array}{l}\text { Age } \\
\text { (years) }\end{array}$ & $\begin{array}{l}\text { Weight } \\
\text { (kg) }\end{array}$ & $\begin{array}{l}\text { BMI } \\
\left(\mathrm{kg} / \mathrm{m}^{2}\right)\end{array}$ & $\begin{array}{l}\text { Dose } \\
(\mathrm{mg} / \mathrm{kg})\end{array}$ & $\begin{array}{l}\text { Total dose } \\
\text { (mg) }\end{array}$ & NIRF imaging of the ureter \\
\hline 1 & $\mathrm{~F}$ & 69 & 61 & 23.24 & 0.50 & 30.5 & $\begin{array}{l}\text { Ureter detected with scope very } \\
\text { close to tissue. } \\
\text { Ureter is also visible in white light } \\
\text { mode }\end{array}$ \\
\hline 2 & $\mathrm{M}$ & 67 & 78 & 26.06 & 0.50 & 40 & $\begin{array}{l}\text { Conversion due to intra-abdominal } \\
\text { adhesions } \\
\text { before NIRF imaging }\end{array}$ \\
\hline 3 & $\mathrm{M}$ & 68 & 98 & 31.64 & 0.25 & 25 & $\begin{array}{l}\text { Left ureter detectable with scope } \\
\text { very close to } \\
\text { tissue, but weak signal. Ureter is also } \\
\text { visible in } \\
\text { white light mode }\end{array}$ \\
\hline 4 & $\mathrm{~F}$ & 87 & 66 & 23.04 & 0.125 & 8 & $\begin{array}{l}\text { Left ureter detectable with scope } \\
\text { close to tissue, } \\
\text { but very weak signal. Ureter is also } \\
\text { visible in } \\
\text { white light mode. }\end{array}$ \\
\hline 5 & $\mathrm{~F}$ & 60 & 72 & 27.44 & 0.25 & 18 & $\begin{array}{l}\text { No visualization of ureter in NIRF } \\
\text { mode during the procedure. Ureter is } \\
\text { visible in white light mode. }\end{array}$ \\
\hline 6 & $M$ & 63 & 103 & 32.15 & 0.25 & 26 & $\begin{array}{l}\text { Conversion due to intra-abdominal } \\
\text { adhesions } \\
\text { before NIRF imaging. }\end{array}$ \\
\hline 7 & M & 63 & 89 & 31.53 & 0.25 & 22 & $\begin{array}{l}\text { No visualization of ureter in } \\
\text { fluorescence mode. } \\
\text { Ureter is visible in white light mode. }\end{array}$ \\
\hline 8 & $\mathrm{M}$ & 67 & 120 & 37.04 & 0.25 & 30 & $\begin{array}{l}\text { No visualization of ureter during the } \\
\text { procedure. } \\
\text { Ureter is visible in white light mode. }\end{array}$ \\
\hline 9 & $\mathrm{M}$ & 69 & 70 & 25.10 & 0.75 & 52.5 & $\begin{array}{l}\text { After } 45 \text { min: good visualization of } \\
\text { both right and } \\
\text { left ureter. In left ureter, better } \\
\text { visualization of } \\
\text { peristaltic movements. Ureter is also } \\
\text { visible in } \\
\text { white light mode. } \\
\text { After } 60 \text { min: good visualization of } \\
\text { left ureter. } \\
\text { After } 90 \text { min: good visualization of } \\
\text { left ureter. }\end{array}$ \\
\hline 10 & $\mathrm{M}$ & 52 & 90 & 25.74 & 1.00 & 90 & $\begin{array}{l}\text { After } 30 \text { min: good visualization of } \\
\text { ureter. Ureter is also visible in white } \\
\text { light mode. } \\
\text { After } 50 \text { min: pulsatile movement of } \\
\text { urine through ureter. }\end{array}$ \\
\hline
\end{tabular}


Table 3 Target-to-Background Ratio measurement for urine samples from patients 5-8 collected for the urine catheter at the end of the surgical procedure

\begin{tabular}{ll}
\hline Patient & Target-to-background ratio \\
\hline 5 & 6.88 \\
\hline 6 & 8.81 \\
\hline 7 & 7.15 \\
8 & 9.20 \\
\hline
\end{tabular}

(Target-to-background ratio: target = urine in vial; background is saline in vial)

Table 4 Target-to-Background Ratio for different dilutions of methylene blue in urine of two healthy volunteers

\begin{tabular}{|c|c|c|c|}
\hline Sample No. & $\begin{array}{l}\text { Dilution } \\
\text { MB:Urine }\end{array}$ & $\begin{array}{l}\text { TBR urine } \\
\text { sample } 1\end{array}$ & $\begin{array}{l}\text { TBR urine } \\
\text { sample } \mathbf{2}\end{array}$ \\
\hline 1 & $1: 1$ & -0.64 & -0.64 \\
\hline 2 & $1: 2$ & -0.196 & 1.04 \\
\hline 3 & $1: 4$ & 0.18 & 3.32 \\
\hline 4 & $1: 8$ & 0.76 & 4.12 \\
\hline 5 & $1: 16$ & 5.58 & 5.82 \\
\hline 6 & $1: 32$ & 8.23 & 8.21 \\
\hline 7 & $1: 160$ & 9.40 & 8.88 \\
\hline 8 & $1: 800$ & 10.33 & 8.52 \\
\hline 9 & $1: 4000$ & 10.33 & 9.27 \\
\hline 10 & $1: 20000$ & 9.90 & 9.30 \\
\hline 11 & $1: 100000$ & 8.70 & 8.53 \\
\hline 12 & $1: 500000$ & 5.08 & 6.88 \\
\hline
\end{tabular}

MB: methylene blue; TBR: target-to-background ratio (target = urine in vial; background is saline in vial)

\section{DISCUSSION}

Various recent studies have paid attention to the use of NIRF imaging in minimally invasive surgery. ${ }^{15}$ This report describes a clinical feasibility study investigating the utility of NIRF imaging using MB for ureteral recognition during human laparoscopic colorectal surgery. Previously, promising results were reported with $\mathrm{MB}$ in an animal model10 and in two human studies during open and laparoscopic surgical procedures. ${ }^{12,16}$ The primary objective of this study was to investigate the feasibility of intraoperative NIRF imaging using MB administration for detection of the left ureter during laparoscopic colorectal surgery.

In this study, it was found that this type of imaging provides additional visual distinction of the left ureter from its surrounding tissues, compared to the conventional laparoscopic view. However, this signal was not strong enough to support the surgeon with enhanced ureteral visualization over the conventional white light mode. When the ureter was identified in fluorescent mode, it could already be seen in white light mode. Another disadvantage is the close proximity (few centimeters) that was required between the endoscope and the ureter to obtain a signal. In the aforementioned previous studies, the authors described 
a distance of 18 and $30 \mathrm{~cm}$ between the camera and the surgical field in open surgery. No distance was given for the laparoscopic experiments. ${ }^{10}$

In this study, higher doses of MB resulted in better visualization of the ureter. The dose chosen in the beginning of our study was based on the study of Matsui et al. ${ }^{10}$ The better recognition with higher doses is in accordance with the observations of Verbeek et al. ${ }^{14}$

The ex vivo experiments with MB dilutions in urine of healthy volunteers showed a strong fluorescence signal in a wide range of dilutions (Table 3), revealing that even very small concentrations of methylene blue in urine can give a clearly visible fluorescence signal. This confirms the possibility to obtain a good signal using the present equipment. Also, in the collected urine from four patients at the end of the surgical procedure, we were able to clearly obtain a fluorescent signal in these urine samples, even though in vivo ureteral detection in these patients was not possible with NIRF imaging. It is hypothesized that the absence of a clear fluorescence signal in the included cases may be influenced by anatomical barriers, such as the ureteral wall thickness and the fatty tissues surrounding the ureter, hindering fluorescence signal detection. Currently, fluorescence imaging at the NIR range of wavelengths has the potential of a better tissue penetration than visible light, up to $10 \mathrm{~mm}$, but the in vivo situation often exceeds this tissue thickness. ${ }^{17}$

The results found in this study are not compatible with the results found in the study by Matsui et al. ${ }^{10}$ in which the feasibility of methylene blue application in open surgery in 20 pigs was investigated. In all animals, the ureter was identified successfully. A possible explanation for these results is the thinner ureteral wall and sub-retroperitoneal layer in pigs and less intra-abdominal fat compared to humans. Verbeek et al. found similar results in humans. ${ }^{10,12}$ However, in their study, they only investigated the feasibility of the use of methylene blue in open surgery. A clear identification of the ureters was observed at doses between 0.25 and $1.0 \mathrm{mg} / \mathrm{kg}$. Perhaps, in the setup of this study, the optimal combination of dye (e.g., dosage and timing of administration) and laparoscopic system has not yet been reached to obtain similar results.

There were no adverse (immediate or delayed) reactions with regard to the clinical outcome following MB administration in this study. However, shortly after the administration of the dye intravenously, a transient decrease of the oxygen saturation was observed, which was measured by a finger pulse oximeter. This phenomenon is known and is caused by the principle of pulse oximetry, which is based on the red and infrared light absorption characteristics of oxygenated and deoxygenated hemoglobin, which is influenced by the transient passage of $\mathrm{MB}^{18}$. Preoperatively, the patient should be notified that intravenous $\mathrm{MB}$ administration could potentially cause a severe anaphylactic reaction; however, this risk is very low to negligible.

Main limitation of this study is that the study population is too small to draw firm conclusions regarding variables such as optimal timing and dosage of dye administration. Furthermore, the patients were included over a relatively long time-span. This was due to 
changing of the involved researchers. This is not likely to have had a negative effect on the performance of the study.

In the cases where the ureter was recognized using the fluorescence mode of the laparoscope, recognition was only possible after the ureter had already been identified using the conventional white light mode, suggesting that fluorescence imaging of the ureter using MB does not provide any added clinical value to this procedure. Therefore, this particular imaging modality in this setup is not yet deemed to be suitable in aiding the identification of the ureter during laparoscopic colorectal surgery.

In conclusion, we report the human clinical experience with near-infrared fluorescence imaging and methylene blue during laparoscopic colorectal surgery for intraoperative ureteral detection. Although promising, as indeed visualization of the ureter could be obtained, this imaging modality did not provide added value in the delineation of the ureter because simultaneous with the fluorescent signal, the ureter could be visualized even in conventional white light mode. Future research should focus on exploring alternative fluorescent dyes (for example preclinical dyes19) and optimization of the equipment to make this promising technique clinically relevant. 


\section{REFERENCES}

1. Stassen LP, Bemelman WA, Meijerink J. Risks of minimally invasive surgery underestimated: A report of the Dutch Health Care Inspectorate. Surg Endoscopy 2010;24: 495-498.

2. Delacroix SE, Jr., Winters JC. Urinary tract injuries: Recognition and management. Clin Colon Rectal Surg 2010; 23:221.

3. Park JH, Park JW, Song K, Jo MK. Ureteral injury in gynecologic surgery: A 5-year review in a community hospital. Korean J Urol 2012;53:120-125.

4. Palaniappa NC, Telem DA, Ranasinghe NE, Divino CM. Incidence of iatrogenic ureteral injury after laparoscopic colectomy. Arch Surg 2012;147:267-271.

5. da Silva G, Boutros M, Wexner SD. Role of prophylactic ureteric stents in colorectal surgery. Asian J Endosc Surg 2012;5:105-110.

6. Assimos DG, Patterson LC, Taylor CL. Changing incidence and etiology of iatrogenic ureteral injuries. J Urol 1994;152(6 Pt 2):2240-2246.

7. Brandes S, Coburn M, Armenakas N, McAninch J. Diagnosis and management of ureteric injury: An evidencebased analysis. BJU Int 2004;94:277-289.

8. Speicher PJ, Goldsmith ZG, Nussbaum DP, Turley RS, Peterson AC, Mantyh CR. Ureteral stenting in laparoscopic colorectal surgery. J Surg Res 2014;190:98-103.

9. Beraldo S, Neubeck K, Von Friderici E, Steinmuller L. The prophylactic use of a ureteral stent in laparoscopic colorectal surgery. Scand J Surg 2013;102:87-89.

10. Matsui A, Tanaka E, Choi HS, Kianzad V, Gioux S, Lomnes SJ, et al. Real-time, near-infrared, fluorescenceguided identification of the ureters using methylene blue. Surgery 2010;148:7886.

11. Tanaka E, Ohnishi S, Laurence RG, Choi HS, Humblet V, Frangioni JV. Real-time intraoperative ureteral guidance using invisible near-infrared fluorescence. J Urol 2007;178: 2197-2202.

12. Verbeek FP, van der Vorst JR, Schaafsma BE, Swijnenburg RJ, Gaarenstroom KN, Elzevier HW, et al. Intraoperative near infrared fluorescence guided identification of the ureters using low dose methylene blue: A first in human experience. J Urol 2013;190:574-579.

13. Schols RM, Bouvy ND, Masclee AA, van Dam RM, Dejong CH, Stassen LP. Fluorescence cholangiography during laparoscopic cholecystectomy: A feasibility study on early biliary tract delineation. Surg Endosc 2013;27:1530- 1536.

14. Verbeek FP, Schaafsma BE, Tummers QR, van der Vorst JR, van der Made WJ, Baeten Cl, et al. Optimization of nearinfrared fluorescence cholangiography for open and laparoscopic surgery. Surg Endosc 2014;28:1076-1082.

15. Schols RM, Connell NJ, Stassen LP. Near-infrared fluorescence imaging for real-time intraoperative anatomical guidance in minimally invasive surgery: A systematic review of the literature. World J Surg 2015;39:1069-1079.

16. Yeung TM, Volpi D, Tullis ID, Nicholson GA, Buchs N,Cunningham C, et al. Identifying Ureters In Situ Under Fluorescence During Laparoscopic and Open Colorectal Surgery. Ann Surg 2016;263:e1-e2.

17. Schols RM, Bouvy ND, van Dam RM, Stassen LP. Advanced intraoperative imaging methods for laparoscopic anatomy navigation: An overview. Surg Endosc 2013;27: 1851-1859.

18. Kessler MR, Eide T, Humayun B, Poppers PJ. Spurious pulse oximeter desaturation with methylene blue injection. Anesthesiology 1986;65:435-436.

19. Schols RM, Lodewick TM, Bouvy ND, van Dam DA, Meijerink WJ, van Dam GM, et al. Nearinfrared fluorescence laparoscopy of the cystic duct and artery in pigs: Performance of a preclinical dye. J Laparoendosc Adv Surg Tech A 2014;24:318-322. 
CHAPTER 9

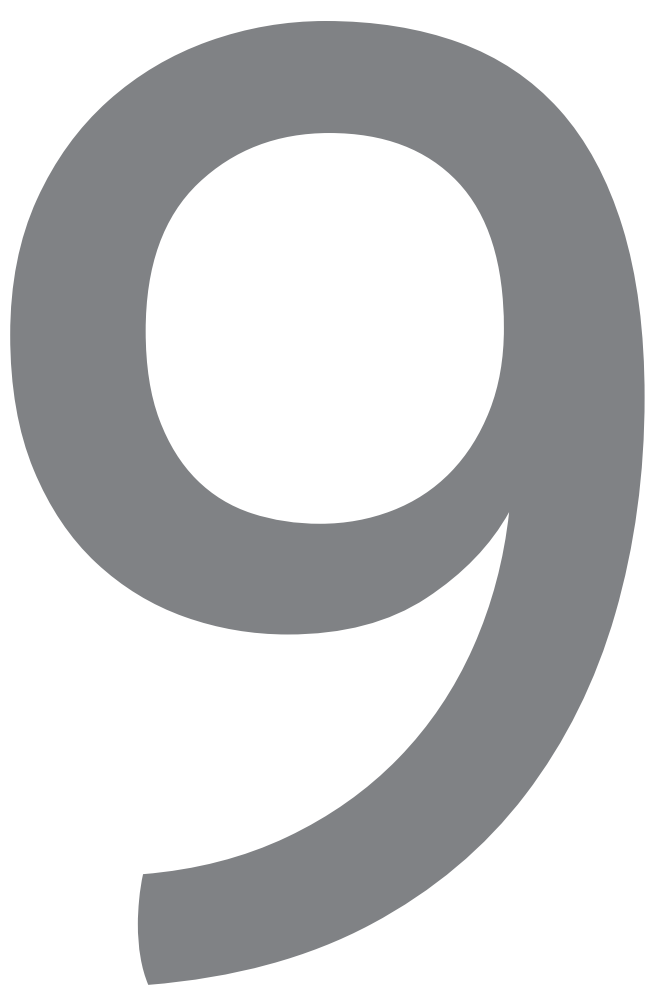




\section{Near-infrared fluorescence laparoscopy of the ureter with three preclinical dyes in a pig model}

Jacqueline van den Bos*, Mahdi Al-Taher*, Nicole D. Bouvy, Laurents P.S. Stassen. Surgical Endoscopy 2019 33(3):986-991

* Both authors contributed equally to the manuscript and therefore share the position of first authorship 


\section{ABSTRACT}

\section{Background}

Ureteric injury is reported to occur in $1-7.6 \%$ of colorectal surgeries. To reduce the incidence of ureteral injury, it is essential to identify the ureters. The use of near-infrared fluorescence (NIRF) imaging with intravenously administered dyes might be of added value for ureteral visualization during laparoscopy. The aim of this study is to assess the performance of three preclinical dyes; IRDye ${ }^{\circledR} 800 \mathrm{BK}$, IRDye ${ }^{\circledast} 800 \mathrm{NOS}$ and IRDye ${ }^{\circledR} 800 \mathrm{CW}$, for near-infrared fluorescence laparoscopy of the ureter in pigs.

\section{Methods}

In three female Dutch landrace pigs, the new dyes were evaluated. In each pig, 1 dye was tested using a 6-mg intravenous dose in a concentration of $1 \mathrm{mg} / \mathrm{ml}$. Imaging was performed in fluorescence mode and white light mode with a laparoscopic imaging system. In order to further evaluate the dyes, an ex vivo imaging experiment was performed, in which 8 decreasing concentrations per dye, diluted in PBS, were evaluated in a transparent test tube with NIRF mode at a distance of 1,5 and $10 \mathrm{~cm}$ from the laparoscope.

\section{Results}

All three dyes were effective in allowing the identification of the ureter with NIRF imaging. The ureter became fluorescent after 35, 45 and 10 min, respectively, for IRDye ${ }^{\circledR} 800 \mathrm{BK}$, IRDye ${ }^{\oplus} 800$ NOS and IRDye ${ }^{\oplus} 800 \mathrm{CW}$ with a maximum target-to-background ratio (TBR) of $2.14,0.66$ and 1.44 , respectively. In the ex vivo imaging experiment, all three dyes produced a strong fluorescence signal at all concentrations and all distances evaluated.

\section{Conclusions}

Intravenous administration of the preclinical dyes IRDye ${ }^{\circledR} 800 C W$, IRDye ${ }^{\circledR} 800$ BK and IRDye ${ }^{\circledast}$ 800 NOS facilitated successful identification of the anatomical course of the ureter in living pig models. The highest measured TBR occurred with the use of IRDye ${ }^{\circledast} 800 B K$. Ex vivo, a correlation was observed between the fluorescence intensities of the signal with the concentration of the dye and with the distance to the object. 


\section{INTRODUCTION}

With a reported incidence rate of $1 \%$ up to $7.6 \%$, the occurrence of ureteric injury is one of the feared complications in colorectal surgery. ${ }^{1-4}$ Ureteric injury can result in pain, intraabdominal sepsis, systemic infection, abscesses, urinoma, ureteral stricture, ureteric fistula, renal failure and loss of the ipsilateral renal unit. ${ }^{4-6}$

Failure to identify the relevant anatomy seems to be the main factor leading to ureter damage. 7 To avoid ureteric injury, it is therefore essential to identify both ureters during surgery. However, this may be difficult and time-consuming. Ureter stent placement is a technique which can be used in open surgery to help identify the ureters. ${ }^{4}$ In laparoscopic surgery, however, in which tactile feedback is limited, this application is hardly useful. A technique improving the visualization of ureters in laparoscopic surgery is therefore needed. The use of near-infrared fluorescence (NIRF) imaging might be able to meet this need. This technique is already used in hepatobiliary surgery to visualize the bile ducts ${ }^{8}$ or the perfusion of the liver ${ }^{9}$, and in colorectal surgery to assess the perfusion of the bowel anastomosis. ${ }^{10-15}$ A few studies have also been published on the use of NIRF imaging to identify the ureters.

The most commonly used fluorescent dye in laparoscopic surgery is indocyanine green (ICG). However, administering this dye intravenously does not facilitate the visualization of the ureters, as it is exclusively cleared by the liver, and therefore not excreted via the urine. ${ }^{16}$ Alternatively, ICG might have potential for visualizing the ureters if administered through a ureter stent. ${ }^{17,} 18$ Disadvantages of this technique include its invasiveness, the requirement of cystoscopy and possible complications such as urinary tract infections, hydronephrosis and hematuria. ${ }^{4}$ This indicates that the use of ICG is a less than optimal option for the visualization of ureters during laparoscopy.

The above illustrates that there is a need for a potent fluorescent dye that can be administered intravenously, is cleared by the kidneys and has no side effects. A previous study showed that the preclinical IRDye ${ }^{\circledast} 800 \mathrm{CW}$ (LI-COR Inc., Lincoln, NE, USA) provided clear ureter visualization in a porcine mode ${ }^{19}$ and, as such, has the potential to be such a dye. As this dye is quite expensive for this application, the manufacturer of IRDye ${ }^{\oplus} 800 \mathrm{CW}$ developed two new, less expensive dyes: IRDye ${ }^{\circledast} 800$ BK and IRDye ${ }^{\oplus} 800$ NOS. These dyes proved to be very promising in cystic duct and cystic artery visualization after intravenous administration. ${ }^{20}$ They are both partially cleared by the liver and by the kidneys, which makes them eligible to be used in NIRF imaging of the ureters after intravenous administration.

The aim of this study is to assess the performance of IRDye ${ }^{\oplus} 800 \mathrm{BK}$, IRDye ${ }^{\circledast} 800 \mathrm{NOS}$ and IRDye ${ }^{\oplus} 800 \mathrm{CW}$ in visualizing the ureter in pigs during near-infrared fluorescence laparoscopy. 


\section{MATERIALS AND METHODS}

This study was conducted at the central animal facilities of Maastricht University (Maastricht, The Netherlands). Animals were used in compliance with the regulations of the Dutch legislation concerning animal research, and the study was done according to a protocol that was approved by the local animal ethics committee. A pig model was chosen because of the similarities between pig anatomy and human anatomy and because of earlier successful application of NIRF imaging in pigs. ${ }^{19}$ The experiments were done in three female Dutch landrace pigs (each weighing $40 \mathrm{~kg}$ ).

\section{Laparoscopic fluorescence imaging system}

A laparoscopic fluorescence imaging system (Karl Storz GmbH \&CO. KG, Tuttlingen, Germany) with a xenon- based light source was used. This system enables excitation and detection of all three dyes used in this experiment: IRDye 800CW (KEX/EM $=775 / 796 \mathrm{~nm})$, IRDye ${ }^{\circledR} 800 \mathrm{BK}$ (KEX/EM $=774 / 790 \mathrm{~nm}$ ) and IRDye ${ }^{\circledast} 800 \mathrm{NOS}$ (KEX/ EM $\left.=767 / 786 \mathrm{~nm}\right)$. All procedures were digitally recorded with the built-in recording equipment. The same NIRF imaging settings were used for all three dyes tested.

\section{Characteristics and preparation of the dyes}

IRDye $^{\circledast} 800 \mathrm{CW}$ is a tetrasulphonated heptamethine indocyanine dye. After intravenous injection, it is cleared by the kidneys and excreted into urine. It is also partially cleared by the liver and excreted into bile. It can therefore be used for both bile duct and ureter visualization. Its maximum absorption occurs at $775 \mathrm{~nm}$ and its maximum excitation emission at $796 \mathrm{~nm}$. The molecular weight of IRDye ${ }^{\circledR} 800 \mathrm{CW}$ is $1090.11 \mathrm{Da} .^{21}$

IRDye ${ }^{\oplus} 800$ BK and IRDye ${ }^{\circledast} 800$ NOS are two newly developed dyes. IRDye ${ }^{\circledast} 800 B K$ is a hydrophilic dye with a maximum absorption of $774 \mathrm{~nm}$ and a maximum emission of 790 $\mathrm{nm}$. Because of its hydrophilic nature, it is primarily cleared by the kidneys. This makes the dye especially useful for intraoperative ureter imaging. Nevertheless, some clearance by the liver takes place.

IRDye $^{\oplus} 800$ NOS is less hydrophilic and, as such, primarily cleared by the liver. This theoretically makes it especially useful for visualization of the biliary system. However, this dye is also partially excreted by the kidneys and could there-fore also facilitate the visualization of ureters. The maximum absorption occurs at $767 \mathrm{~nm}$ and its maximum emission at $786 \mathrm{~nm}$.

The dyes were prepared and used following instructions of the manufacturer.

\section{Surgical technique and assessment guidelines}

The surgical procedures were performed under general anesthesia, as has been previously described in an earlier study. ${ }^{20}$ Surgical residents performed a laparoscopic partial excision 
of the bicornate uterus, mimicking a laparoscopic appendectomy. These procedures were strictly supervised by two expert endoscopic gastrointestinal surgeons (NB and LS). Each pig received one of the three dyes intravenously at a total dose of $6 \mathrm{mg}(1 \mathrm{mg} / \mathrm{ml})$.

Imaging was performed intermittently in fluorescence mode and white light mode. Intraoperatively, the first authors (MA and JvdB) systematically documented whether the ureters could be identified in fluorescence mode, by filling in a registration form. The attending surgeon was consulted to reach agreement on the identification of the ureters. A structure was defined as 'identified' if its localization was confirmed with certainty by the experienced surgeon.

\section{Ex vivo NIRF imaging}

In the ex vivo experiment, 8 decreasing dye concentrations, diluted in PBS, were evaluated in NIRF mode, in a completely darkened room with the laparoscope held at a distance of 1,5 and $10 \mathrm{~cm}$, respectively. A transparent $10 \mathrm{ml}$ test tube was filled with $10 \mathrm{ml}$ of each dye concentrations. The initial dilution consisted of $10 \mathrm{mg}$ of the dye being diluted in 10 $\mathrm{ml}$ of PBS. From this basic concentration, decreasing dye concentrations were made. The evaluated concentrations of dye/PBS were 1:1, 1:2, 1:4, 1:8, 1:16, 1:32, 1:64 and 1:128.

\section{Postoperative analysis of the fluorescence}

For an objective assessment of the fluorescence illumination, OSIRIX Lite v9 imaging software (Pixmeo, Geneva, Switzerland) was used. Fluorescence was analyzed by deter- mining the fluorescence intensity (FI) and the target-to-back- ground ratio (TBR). By doing so, it could be objectified whether the target organ (the ureter) was more fluorescent compared to the surrounding tissues. The TBR is defined as the mean fluorescence intensity (FI in arbitrary units, A.U.) of three points of interest in the target (ureter), minus the mean fluorescence intensity of three points of interest in the background divided by the mean fluorescence intensity of three points of interest in the background. In formula: TBR $=$ (FI of target $-\mathrm{FI}$ of background)/(FI background).19, 20, 22 Areas with light scattering were avoided in these points of interest. The background $\mathrm{Fl}$ in the ex vivo experiments was negligible due to the completely darkened room, and therefore, no TBR was calculated.

For the $\mathrm{Fl}$ of the target, three centrally located regions of interest were chosen in the ureter. The mean fluorescence intensity of these three regions was the $\mathrm{Fl}$ of target. When using this technique to establish the TBR, choosing a reproducible background is important. Therefore, as background 3 regions of interest $1 \mathrm{~cm}$ bilateral (2 right and 1 left) from the ureter were chosen in the in-vivo study. The mean scores of these three fluorescence intensities were used as the $\mathrm{Fl}$ of background.

For the ex vivo study, the mean of three regions of interest at the center of the test tube filled with dye dilution was chosen as a target. 


\section{RESULTS}

In all three experiments, it was possible to successfully identify the ureters with NIRF imaging. Results obtained during the operations are presented in the intraoperative registration form in Table 1. A representative screenshot in fluorescence mode was made of each of the three pigs.

The first witnessed identification of the ureters occurred within minutes after dye administration with all three dyes. The ureteral wall became fluorescent in NIRF mode together with the uterus, bowel and lymph nodes. No peristaltic movement of urine within the ureters could yet be seen. Therefore, we could not identify the course of the ureter with absolute certainty at this stage.

Thirty-five minutes after the administration of the IRDye ${ }^{\circledR} 800 \mathrm{BK}$ dye, peristaltic movement of the urine was clearly visible in NIRF mode due to the fluorescent dye excreted in the urine. The ureters remained fluorescent until the final assessment $3.5 \mathrm{~h}$ after dye administration. The highest measured TBR was 2.14 (Figure 1a).

The first appearance of fluorescence of the ureters in the second pig (IRDye ${ }^{\circledR} 800 \mathrm{NOS}$ ) occurred $45 \mathrm{~min}$ after dye administration with clear peristaltic movement of urine within the ureters. The highest measured TBR was 0.66 (Figure 1b).

In the third pig, the first fluorescent imaging of the ureters occurred $10 \mathrm{~min}$ after the IRDye ${ }^{\oplus} 800 C W$ dye was administered. Both ureters were clearly distinguishable from their surroundings. A second evaluation, 25 min after dye administration, showed a persistent clear delineation of the ureters in NIRF mode. The highest measured TBR was 1.44 (Figure 1c).

No complications or adverse reactions were observed in any of the experiments.

\section{Ex vivo NIRF imaging}

With all three dyes, a strong fluorescence signal was achieved in all concentrations and at all distances evaluated. The results are depicted in Figure 2a-c.

At $1 \mathrm{~cm}$ distance, all dyes in all concentrations showed comparable FI without clear fluctuations. At $5 \mathrm{~cm}$ distance, the $\mathrm{Fl}$ of all dyes was lower in the highest concentrations. In these concentrations, IRDye ${ }^{\circledast} 800 \mathrm{CW}$ showed the highest FI. This effect disappeared in the lower concentrations. At $10 \mathrm{~cm}$ distance, also an inverse relation between concentration and FI was observed for all three dyes. IRDye ${ }^{\circledR} 800 \mathrm{CW}$ and IRDye ${ }^{\circledR} 800 \mathrm{BK}$ showed a higher FI in all concentrations when compared to IRDye ${ }^{\circledR} 800$ NOS. All three dyes showed a decrease in $\mathrm{Fl}$ when the laparoscope was held at a $10 \mathrm{~cm}$ distance, as compared to when it was held at 1 and $5 \mathrm{~cm}$ distance, respectively. The highest Fls were achieved at $1 \mathrm{~cm}$ distance for the higher concentrations and at a $5 \mathrm{~cm}$ distance for the lower concentrations. 
Table 1 Intraoperative registration form

\begin{tabular}{|c|c|c|c|c|}
\hline Injected dye & $\begin{array}{l}\text { Visualization } \\
\text { left ureter }\end{array}$ & $\begin{array}{l}\text { Visualization } \\
\text { right ureter }\end{array}$ & $\begin{array}{l}\text { Time to first certain } \\
\text { visualization of } \\
\text { ureter }\end{array}$ & $\begin{array}{l}\text { Highest } \\
\text { measured TBR }\end{array}$ \\
\hline IRDye ${ }^{\circledR} 800 \mathrm{BK}$ & Yes & Yes & $35 \mathrm{~min}$ & 2.14 \\
\hline IRDye ${ }^{\circledast} 800$ NOS & Yes & Yes & $45 \mathrm{~min}$ & 0.66 \\
\hline IRDye ${ }^{\circledast} 800 C W$ & yes & Yes & $10 \mathrm{~min}$ & 1.44 \\
\hline
\end{tabular}

TBR: Target-to-background ratio
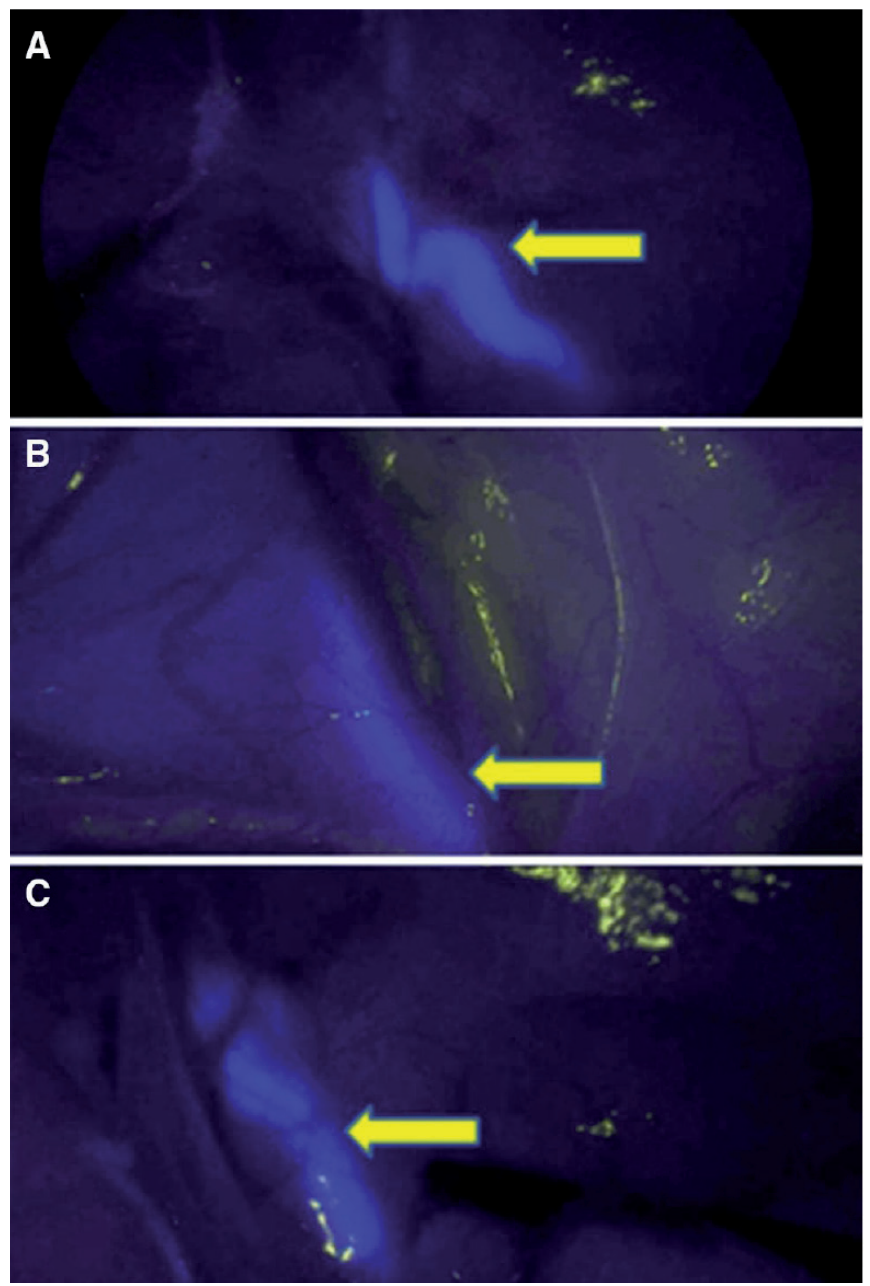

Figure 1 Visualization of the ureter with the experimental dyes: a with IRDye ${ }^{\circledR} 800 B K$ with highest TBR (2.14), b with IRDye ${ }^{\circledast} 800$ NOS with highest TBR (0.66), c with IRDye 800CW with highest TBR (1.44) 

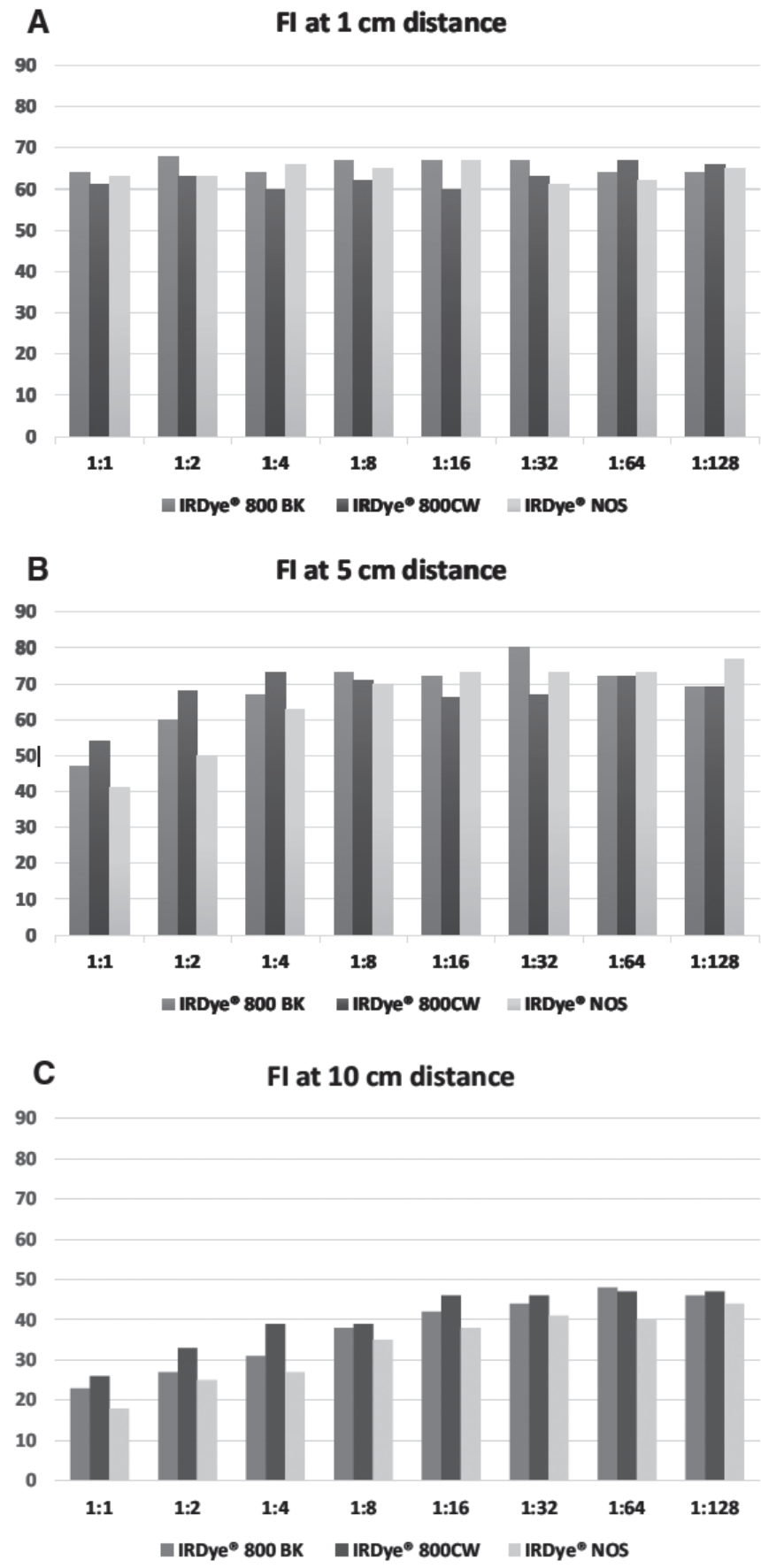

Figure 2 Fluorescence intensities (FI) for the three dyes for different dye concentrations with the laparoscope held at $1 \mathrm{~cm}(\mathrm{~A}), 5 \mathrm{~cm}$ (B) and $10 \mathrm{~cm}$ (C) distance from the object. 


\section{DISCUSSION}

The most frequently used near-infrared fluorescent dye, indocyanine green, is exclusively cleared by the liver ${ }^{20}$ and can therefore not be used for ureteral imaging after intravenous administration. Such imaging requires a dye that is excreted in urine or invasive techniques with a ureter catheter insertion through which the fluorescent dye can be introduced into its lumen. ${ }^{18,23}$

Verbeek et al. used methylene blue as a fluorescent dye for ureter visualization in open pelvic surgery in 12 patients. In all 12 patients, the ureters could be clearly visualized. ${ }^{24}$ Yueng et al. showed visualization of the ureters in 10 out of 11 included patients. The technique was considered useful in 4 of these 10 cases. A recent study by Barnes et al. showed promising results allowing visualization of the ureter in a clinical study in which fluorescence imaging with methylene blue was of added value compared to white light in 14 of 69 ureters assessed..$^{25}$ However, in another study that used methylene blue as a fluorescent dye in laparoscopic colorectal surgery, this technique was not found to be superior to conventional white light laparoscopy in any of the included cases. ${ }^{26}$ Although no side effects are reported in these studies, a disadvantage of using methylene blue is that it comes with some potential side effects, such as a small risk of anaphylactic reaction ${ }^{27}$ and the potential occurrence of vasoconstriction.

A promising near-infrared fluorescent dye that is cleared by the kidneys is IRDye ${ }^{\circledR}$ $800 \mathrm{CW} .{ }^{23}$ However, this dye is expensive for this application. Newer pre-clinical dyes which are cheaper to produce are IRDye ${ }^{\oplus} 800$ NOS and IRDye ${ }^{\oplus} 800 \mathrm{BK}$. The manufacturer estimates their cost to be in the range of commercially available ICG (personal communication). In the present study, the usefulness of three preclinical dyes for NIRF imaging of the ureters was explored. IRDye ${ }^{\circledast} 800 \mathrm{BK}$ is especially developed for excretion via the urine, being hydrophilic in nature. It is therefore expected that this dye will equal or outperform the imaging capabilities that have previously been described for IRDye ${ }^{\circledR} 800 \mathrm{CW}^{20}$

All three dyes enabled clear and satisfactory visualization of the anatomical course of both ureters, as can be seen in Fig. 1a-c. The highest maximum in-vivo FI and TBR was measured with the IRDye ${ }^{\circledast} 800 \mathrm{BK}$, and the lowest maximum FI and TBR was found with the IRDye ${ }^{\oplus} 800$ NOS. This is in line with expectations, as the hydrophilic IRDye ${ }^{\circledast} 800 B K$ is mainly cleared by the kidneys, while IRDye ${ }^{\circledR} 800$ NOS is less hydrophilic and only partially cleared by the kidneys. Nevertheless, all three dyes give clear visibility of the ureters. The TBRs for IRDye ${ }^{\circledR} 800 \mathrm{CW}$ were comparable with previous experiments. ${ }^{19}$

In the ex vivo study, a clear fluorescence signal was achieved with all concentrations and at all the tested distances. The highest Fls were achieved when the laparoscope was held at 1 and $5 \mathrm{~cm}$ distance, whereas the lowest $\mathrm{Fl}$ was achieved at a distance of $10 \mathrm{~cm}$. This supports earlier studies which showed that the Fl is negatively influenced by an increase in the distance of the laparoscope from the target. ${ }^{28}$ 
Further studies evaluating more concentrations and distances may be helpful in identifying the ideal distance/concentration combination.

There were no adverse reactions as a result of the administration of the dyes. A transient decrease in $\mathrm{SpO} 2$ oxygen saturation is known with the use of intravenous fluorescent dyes, temporarily resulting in falsely lower oxygen levels. ${ }^{29,}{ }^{30} \mathrm{An}$ advantage of these new dyes over iodine containing ICG is that these dyes can also be used in patients with a known hypersensitivity to iodine or iodine allergy. Due to the low number of pigs assessed, no conclusion may yet be drawn regarding the safety of the dyes.

Despite the promising results, the findings of this study have to be interpreted with caution. Since each dye was only tested in-vivo at one specific dosage and each dye was only tested in one pig, further experiments are needed to determine optimal dosing and timing of the dyes which are dependent on the pharmacokinetic properties of the dyes. Furthermore, testing in human subjects should be awaited in order to assess the clinical value of the dyes.

\section{CONCLUSION}

Intravenous administration of the preclinical dyes IRDye ${ }^{\circledast} 800 C W$, IRDye ${ }^{\circledast} 800$ BK and IRDye ${ }^{\circledast}$ 800 NOS allowed for successful NIRF identification of the course of the ureters in a live pig model. The use of IRDye ${ }^{\circledast} 800 \mathrm{BK}$ resulted in the highest contrast between ureter and background.

Ex vivo, a correlation of the signal was observed with the concentration of the dye and with the distance to the object. 


\section{REFERENCES}

1. Andersen P, Andersen LM, Iversen LH (2015) latrogenic ureteral injury in colorectal cancer surgery: a nationwide study comparing laparoscopic and open approaches. Surg Endosc 29(6):1406-1412

2. Marcelissen TA, Den Hollander PP, Tuytten TR, Sosef MN (2016) Incidence of iatrogenic ureteral injury during open and laparoscopic colorectal surgery: a single center experience and review of the literature. Surg Laparosc Endosc Percutan Tech 26(6):513-515

3. Zafar SN, Ahaghotu CA, Libuit L, Ortega G, Coleman PW, Cornwell III EE, et al (2014) Ureteral injury after laparoscopic versus open colectomy. JSLS 18(3)

4. da Silva G, Boutros M, Wexner SD (2012) Role of prophylactic ureteric stents in colorectal surgery. Asian J Endosc Surg 5(3):105-110

5. Abboudi H, Ahmed K, Royle J, Khan MS, Dasgupta P, N'Dow J (2013) Ureteric injury: a challenging condition to diagnose and manage. Nat Rev Urol 10(2):108-115

6. Esparaz AM, Pearl JA, Herts BR, LeBlanc J, Kapoor B (2015) latrogenic urinary tract injuries: etiology, diagnosis, and management. Semin Intervent Radiol 32(2):195-208

7. Zhang X, Wang Z, Zhou H, Liang J, Zhou Z (2014) Analysis of ureteral injuries for laparoscopic rectal cancer surgery. J Laparoendosc Adv Surg Tech A 24(10):698-701

8. Osayi SN, Wendling MR, Drosdeck JM, Chaudhry UI, Perry KA, Noria SF et al (2015) Near-infrared fluorescent cholangiography facilitates identi cation of biliary anatomy during laparoscopic cholecystectomy. Surg Endosc 29(2):368-375

9. Narasaki H, Noji T, Wada H, Ebihara Y, Tsuchikawa T, Oka- mura K et al (2017) Intraoperative real-time assessment of liver function with near-infrared fluorescence imaging. Eur Surg Res 58(5-6):235-245

10. Boni L, Fingerhut A, Marzorati A, Rausei S, Dionigi G, Cassi- notti E (2017) Indocyanine green fluorescence angiography dur- ing laparoscopic low anterior resection: results of a casematched study. Surg Endosc 31(4):1836-1840

11. Jafari MD, Lee KH, Halabi WJ, Mills SD, Carmichael JC, Stamos MJ et al (2013) The use of indocyanine green fluorescence to assess anastomotic perfusion during robotic assisted laparoscopic rectal surgery. Surg Endosc 27(8):3003-3008

12. Kin C, Vo H, Welton L, Welton M (2015) Equivocal effect of intraoperative fluorescence angiography on colorectal anastomotic leaks. Dis Colon Rectum 58(6):582-587

13. Kudszus S, Roesel C, Schachtrupp A, Hoer JJ (2010) Intraoperative laser fluorescence angiography in colorectal surgery: a noninvasive analysis to reduce the rate of anastomotic leakage. Langenbecks Arch Surg 395(8):1025-1030

14. Wada T, Kawada K, Takahashi R, Yoshitomi M, Hida K, Hasegawa S et al (2017) ICG fluorescence imaging for quantitative evaluation of colonic perfusion in laparoscopic colorectal surgery. Surg Endosc 31(10):4184-4193

15. Jafari MD, Wexner SD, Martz JE, McLemore EC, Margolin DA, Sherwinter DA et al (2015) Perfusion assessment in laparoscopic left-sided/anterior resection (PILLAR II): a multi-institutional study. J Am Coll Surg 220(1):82-92 e1

16. Levesque E, Martin E, Dudau D, Lim C, Dhonneur G, Azoulay D (2016) Current use and perspective of indocyanine green clearance in liver diseases. Anaesth Crit Care Pain Med 35(1):49-57

17. Lee Z, Simhan J, Parker DC, Reilly C, Llukani E, Lee DI et al (2013) Novel use of indocyanine green for intraoperative, real- time localization of ureteral stenosis during robot-assisted ureteroureterostomy. Urology 82(3):729-733

18. Siddighi S, Yune JJ, Hardesty J (2014) Indocyanine green for intraoperative localization of ureter. Am J Obstet Gynecol 211(4):436 e1-e2

19. Schols RM, Lodewick TM, Bouvy ND, van Dam GM, Dejong CH, Stassen LP (2014) Application of a new dye for near-infrared fluorescence laparoscopy of the ureters: demonstration in a pig model. Dis Colon Rectum 57(3):407-411 
20. van den Bos J, Al-Taher M, Hsien SG, Bouvy ND, Stassen LPS (2017) Near-infrared fluorescence laparoscopy of the cystic duct and cystic artery: first experience with two new preclinical dyes in a pig model. Surg Endosc 31(10):4309-4314

21. Leung K IRDye $800 \mathrm{CW}$-Human serum albumin. Molecular imag- ing and contrast agent database (MICAD). Bethesda (MD)2004

22. Schols RM, Bouvy ND, van Dam RM, Masclee AA, Dejong CH, Stassen LP (2013) Combined vascular and biliary fluorescence imaging in laparoscopic cholecystectomy. Surg Endosc 27(12):4511-4517

23. Lee Z, Moore B, Giusto L, Eun DD (2015) Use of indocyanine green during robot-assisted ureteral reconstructions. Eur Urol 67(2):291-298

24. Verbeek FP, van der Vorst JR, Schaafsma BE, Swijnenburg RJ, Gaarenstroom KN, Elzevier HW et al (2013) Intraoperative near infrared fluorescence guided identification of the ureters using low dose methylene blue: a first in human experience. J Urol 190(2):574-579

25. Barnes TG, Hompes R, Birks J, Mortensen NJ, Jones O, Lindsey I et al (2018) Methylene blue fluorescence of the ureter during colorectal surgery. Surg Endosc 32(9):4036-4043

26. Al-Taher M, van den Bos J, Schols RM, Bouvy ND, Stassen LP (2016) Fluorescence ureteral visualization in human laparoscopic colorectal surgery using methylene blue. J Laparoendosc Adv Surg Tech A 26(11):870-875

27. Ginimuge PR, Jyothi SD (2010) Methylene blue: revisited. J Anaesthesiol Clin Pharmacol 26(4):517-520

28. Kono Y, Ishizawa T, Tani K, Harada N, Kaneko J, Saiura A et al (2015) Techniques of fluorescence cholangiography during laparoscopic cholecystectomy for better delineation of the bile duct anatomy. Medicine (Baltimore) 94(25):e1005

29. Baek HY, Lee HJ, Kim JM, Cho SY, Jeong S, Yoo KY (2015) Effects of intravenously administered indocyanine green on near- infrared cerebral oximetry and pulse oximetry readings. Korean $J$ Anesthesiol 68(2):122-127

30. Ediriwickrema LS, Francis JH, Arslan-Carlon V, Dalecki PH, Bro- die SE, Marr BP et al (2015) Intravenous injection of indocyanine green results in an artificial transient desaturation by pulse oxime- try. Retin Cases Brief Rep 9(3):252-255 
CHAPTER 10

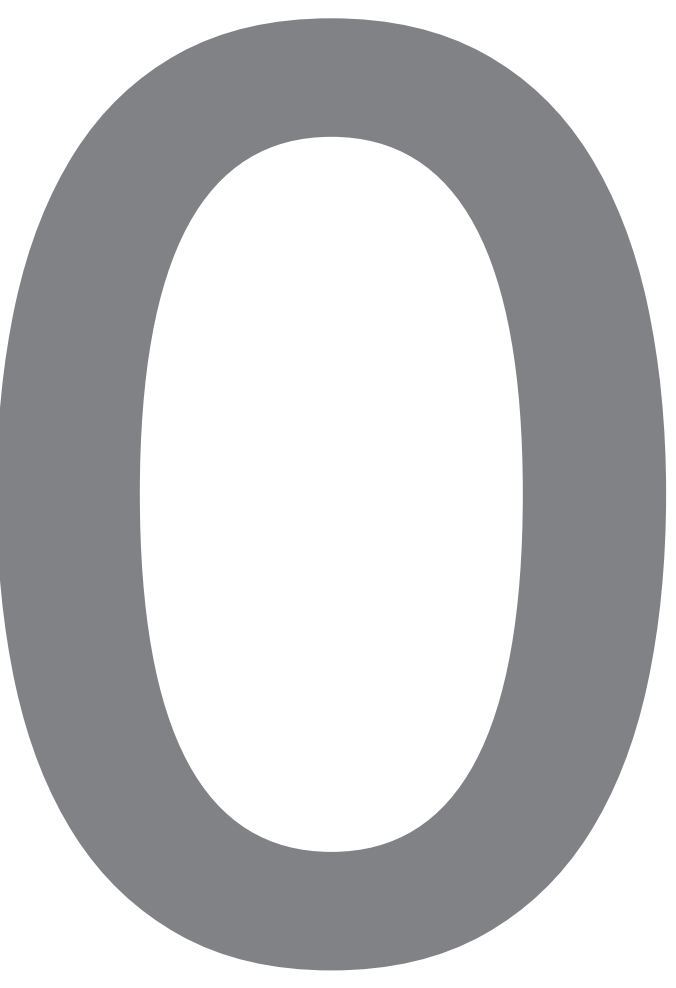




\section{Evaluation of a novel dye for near- infrared fluorescence delineation of the ureters during laparoscopy}

Mahdi Al-Taher, Jacqueline van den Bos, Rutger M. Schols, Bela Kubat, Nicole D. Bouvy, Laurents P.S. Stassen.

BJS Open 2018 DOI: 10.1002/bjs5.59 


\section{ABSTRACT}

\section{Background}

latrogenic ureteric injury remains a risk in laparoscopic pelvic procedures. Near-infrared fluorescence (NIRF) imaging is a promising new technique for enhanced intraoperative visualization of anatomical structures that could improve the safety of laparoscopic surgery. A new fluorescent dye, IRDye ${ }^{\oplus} 800$ BK has been developed for intraoperative visualization of the ureters using NIRF. The present study was a first evaluation of the performance of IRDye ${ }^{\oplus}$ $800 \mathrm{BK}$ for ureteric imaging during NIRF laparoscopy.

\section{Methods}

This study consisted of three parts: real-time in vivo NIRF imaging using IRDye ${ }^{\oplus} 800$-BK in pigs during laparoscopic surgery, ex vivo NIRF imaging of freshly explanted pig ureters and ex vivo NIRF imaging of explanted human ureters.

\section{Results}

In all animals, both left and right ureters were visualized throughout the laparoscopic procedure for $120 \mathrm{~min}$, with the best results at a dose of $0.15 \mathrm{mg}$ dye per $\mathrm{kg}$ bodyweight. NIRF imaging was successful in all human and porcine ureters studied, with a range of dye concentrations.

\section{Conclusions}

NIRF imaging of the ureters using IRDye ${ }^{\oplus} 800$-BK was used successfully both in vivo in a porcine model, and ex vivo in porcine and human ureters. 


\section{INTRODUCTION}

Despite advances in laparoscopic surgery, iatrogenic ureteric injuries during laparoscopy may still occur. Several studies ${ }^{2-5}$ have reported an incidence of between 0.1 and 7.6 per cent in colorectal and gynecological surgery, with more than 80 per cent of injuries going unrecognized during surgery. Ureteric injury leads to significant postoperative morbidity even if identified and repaired during the same procedure. ${ }^{6}$ Near-infrared fluorescence (NIRF) imaging is a promising new technique for easier and earlier intraoperative visualization of target organs with the potential to improve the safety of laparoscopic surgery. ${ }^{7,8}$ Indocyanine green (ICG) and methylene blue are currently the only clinically available dyes that may be helpful in intraoperative visualization of the ureter. ${ }^{9-11}$ However, a feasibility study ${ }^{12}$ recently showed that, even though imaging using methylene blue was safe and feasible, it did not provide a practical advantage over conventional laparoscopic imaging for identification of the ureter during laparoscopic colorectal surgery.

IRDye ${ }^{\circledR} 800 \mathrm{CW}$ (LI-COR Biosciences, Lincoln, Nebraska, USA) is an experimental dye that allows intraoperative visualization of crucial anatomical structures using NIRF imaging. Animal studies have successfully demonstrated the potential of this dye for identification of the ureters. ${ }^{13-15}$ However, a major disadvantage of IRDye ${ }^{\circledR} 800 \mathrm{CW}$ is its cost, which is almost tenfold that of ICG.

Recently a new preclinical dye, IRDye ${ }^{\circledast} 800$-BK (LI-COR Biosciences), has been developed specifically for intraoperative NIRF visualization of the ureters. Because of its hydrophilic nature, it is primarily cleared by the kidneys and may therefore have great potential for realtime NIRF imaging of the ureter. According to the manufacturer, the price of IRDye ${ }^{\circledR} 800$-BK is expected to be similar to that of ICG. The aim of the present study was to evaluate this dye for ureteric imaging during NIRF laparoscopy.

\section{METHODS}

This study was conducted at the central animal facilities of Maastricht University (Maastricht, The Netherlands). Animals were used in compliance with the regulations of Dutch legislation for animal research and following a protocol approved by the local animal ethics committee. Three female Dutch Landrace pigs were used as well as explanted porcine and human ureters. The ARRIVE guidelines ${ }^{16}$ were followed for reporting these experiments.

A laparoscopic fluorescence imaging system (Karl Storz, Tuttlingen, Germany) was used. The D-Light $\mathrm{P}$ system includes a plasma light guide and a 30degree $10-\mathrm{mm}$ laparoscope applicable for white light and NIRF imaging. It enables excitation of the dye under evaluation. A foot pedal allows the surgeon to switch easily between the two imaging modalities. All procedures were recorded digitally. 
IRDye ${ }^{\circledR} 800$ BK has a maximum absorption at $774 \mathrm{~nm}$ and a maximum emission at 790 $\mathrm{nm}$. The dye was prepared before use according to the manufacturer's instructions and diluted in sterile phosphate-buffered saline (PBS) to a concentration of $1 \mathrm{mg} / \mathrm{ml}$.

The experiments consisted of three parts. The performance of the dye was tested in vivo in a pig model, as a dose-finding study. Then, the influence of the thickness of the ureter wall and the dose administered on the signal achieved was tested during ex vivo experiments using both porcine and human ureters.

\section{In vivo fluorescence imaging}

The dye was tested during laparoscopic surgery in three female Landrace pigs, each weighing between 39 and $39.6 \mathrm{~kg}$. Premedication consisted of intramuscular injection of azaperone $3 \mathrm{mg} / \mathrm{kg}$, ketamine $10 \mathrm{mg} / \mathrm{kg}$ and atropine $0.05 \mathrm{mg} / \mathrm{kg}$. Anesthesia was induced with intravenous thiopental $10-15 \mathrm{mg} / \mathrm{kg}$. After intubation, the pigs were maintained under anesthesia with isoflurane and oxygen. Vital parameters were monitored continuously.

The pigs were placed supine in a steep Trendelenburg position and three trocars were introduced: a 12- $\mathrm{mm}$ trocar in the midline superior to the umbilicus and two 5- $\mathrm{mm}$ trocars on either side. Pneumoperitoneum was established with carbon dioxide. Surgery and NIRF imaging were performed by two surgeons specifically experienced in laparoscopic surgery and NIRF imaging. The ureters were not dissected or exposed in any animal.

The first pig received an intravenous dose of $6 \mathrm{mg}(6 \mathrm{ml})$ IRDye ${ }^{\circledR} 800 \mathrm{BK}$ as a bolus, which was in the range of doses used in previous experiments with IRDye ${ }^{\circledR} 800 \mathrm{CW}$. The second and third pigs received half $(3 \mathrm{mg})$ and double $(12 \mathrm{mg})$ this dose respectively. The dye was administered directly after introduction of the laparoscopic trocars. Observation of the left and right ureters, in white light and fluorescence mode, was planned for every 10 min for $1 \mathrm{~h}$, starting after $20 \mathrm{~min}$, and every $20 \mathrm{~min}$ during the second hour. However, the ureter was visualized clearly after $20 \mathrm{~min}$ in the first pig, so it was decided to initiate visualization right from the start of the operation in the next two animals. After surgery, the pigs were killed in accordance with the guidelines of the Dutch legislation for animal research.

\section{Ex vivo fluorescence imaging of porcine ureters}

Freshly explanted pig ureters were collected from the abattoir on the day of the experiments. Ureters were flushed with PBS followed by flushing with the diluted IRDye ${ }^{\circledast} 800$-BK, during which first the distal and then the proximal part of the ureter was clamped, ensuring watertight closure on both sides. The whole process was recorded with the laparoscope in fluorescence mode at a distance of $10 \mathrm{~cm}$ from the ureter. The same background was used in all ureters studied. The following dilutions were examined: 1:4, 1:16, 1:64 and 1:256.

\section{Ex vivo fluorescence imaging of human ureters}

Tissue was obtained from the Maastricht Pathology Tissue Collection. Collection, storage, 
and use of tissue and patient data were in agreement with the code for proper secondary use of human tissue in the Netherlands (http://www.federa.org). A pathologist explanted the ureters during post-mortem examination of patients not previously known to have urological diseases or ureteric abnormalities. All ureters were explanted within 2 weeks before experimentation and stored at -20 degrees Celsius. They were defrosted at room temperature $3 \mathrm{~h}$ before the experiments. The NIRF imaging procedure was similar to that for ex vivo porcine ureters. Dilutions examined were: 1:1, 1:4, 1:16, 1:64, 1:256, 1:1024 and 1:4096.

\section{Measurement of ureter wall thickness}

In ex vivo experiments, a representative cross-section of the distal third of the ureter was analyzed microscopically, on the basis that this part of the ureter is usually of interest during laparoscopic colorectal and gynecological surgery. ${ }^{17}$ Ureter wall thickness was defined as the distance from the surface of the luminal urothelium to the outer layer of smooth muscle. Wall thickness values presented are the mean of nine measurements per cross-section.

\section{Analysis}

Video recordings of the laparoscopic procedures were assessed for the degree of fluorescence illumination using OSIRIX version 5.0.1 imaging software (Pixmeo, Geneva, Switzerland). Signal and target-to-background ratio (TBR) were determined from representative screenshots. The TBR was defined as the mean fluorescence intensity (FI) of three points of interest in the target (ureter) minus the mean $\mathrm{Fl}$ of three points of interest in the background directly adjacent to the ureter, divided by the mean Fl of three points of interest in the background. ${ }^{18}$

The ex vivo experiments were performed in a completely darkened room with the laparoscope $10 \mathrm{~cm}$ from the explanted ureter. The fluorescence from an area $1 \mathrm{~cm}$ lateral to the ureter was chosen as the background fluorescence for each ureter.

\section{RESULTS}

\section{In vivo fluorescence imaging}

Pig 1, with a bodyweight of $39.4 \mathrm{~kg}$, received an intravenous bolus of $6 \mathrm{mg}$ dye, resulting in a dose of $0.15 \mathrm{mg}$ dye per $\mathrm{kg}$ bodyweight. The first clear and distinct visualization of both the left and right ureters in fluorescence mode occurred 20 minutes after administration of the dye. Pig 2, with a bodyweight of $39.0 \mathrm{~kg}$, received an intravenous bolus of $3 \mathrm{mg}$ dye (0.08 $\mathrm{mg}$ dye per $\mathrm{kg}$ bodyweight). The first clear and distinct visualization of both ureters in fluorescence mode occurred 20 minutes after dye administration. Pig 3, with a bodyweight of $39.6 \mathrm{~kg}$, received an intravenous bolus of $12 \mathrm{mg}$ dye $(0.30 \mathrm{mg}$ per kg bodyweight). The first visualization of both ureters in fluorescence mode occurred 1 minute after administration 
of dye. At this stage, it was the wall of both ureters that became clearly visible. The first peristaltic movements of urine through the ureter were observed 10 minutes after administration of dye.

In all three pigs, both the left and right ureter were visualized with peristaltic movement until the end of the experiment after 120 minutes, confirming transportation of the dyecontaining urine through the ureter. A very clear and certain course of the ureter could be observed subjectively in pigs 1 and 3. In contrast, the Fl of the ureter in pig 2 was significantly lower than in the other two pigs, making it difficult to distinguish the ureter from the surrounding tissues. The measured Fl is shown in Figure 1. Pig 1 had the highest absolute FI measured, followed by pig 3 and pig 2. Figure 2 shows TBRs at various points during the laparoscopy for the left ureter in the three pigs. The TBR was highest for the highest doses administered.

In all pigs, the subjective maximum intensity of the fluorescence signal was seen at moments of peristaltic contractions of the ureter, showing transport of intraluminal urine from proximal to distal. Screenshots of the left ureter 30 and $120 \mathrm{~min}$ after injection of $6 \mathrm{mg}$ IRDye $^{\circledast} 800$-BK in pig 1 are shown in Figure 3, with the ureter distinct from the surrounding tissue.

\section{Ex vivo fluorescence imaging of porcine ureters}

The wall thickness of the four pig ureters ranged from 93.3 to 129.0 (mean 113.6) $\mu \mathrm{m}$. A clear delineation of all four ureters was possible using fluorescence imaging, even the lowest concentration of dye tested. FI and TBR ranged from 38 to 56 and 6.60 to 10.20 respectively (Table 1). An initial lowering of the concentration seemed to improve the signal, despite increasing wall thickness (TBR ureter 2 versus 1). Further dilution gave varying results: an initial decrease in the signal, as observed in ureter 3, but an increase in ureter 4 . Variations in concentration and wall thickness are shown in Table 1. A further observation was that fatty tissue on the ureter strongly influenced the fluorescence signal (Figure 4). A signal could be obtained only in areas where the ureter was not covered by fat as seen by the naked eye. This effect was consistent across all ureters and at all concentrations tested.

After rinsing the ureter extensively with pure PBS, a low fluorescence signal remained in the ureter, both in the lumen and in the outer layer of the ureter (Figure 5), suggesting uptake of the dye in the wall of the ureter. 


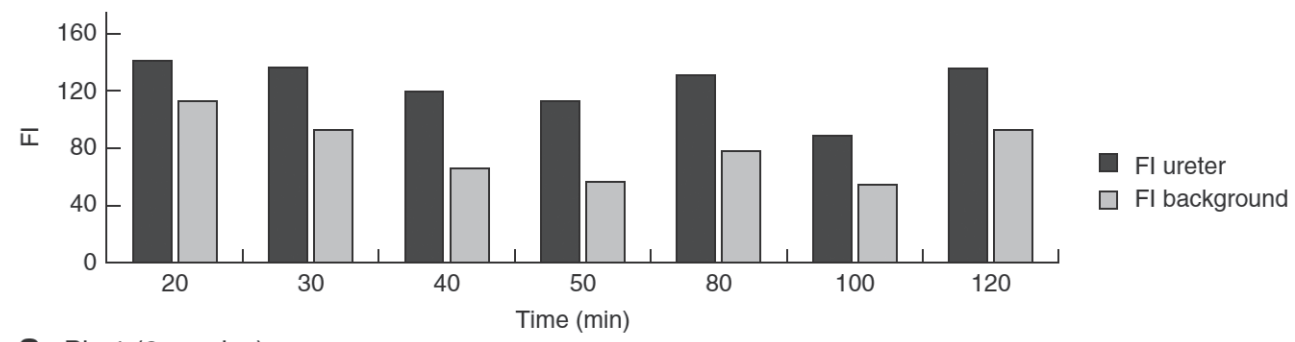

a $\operatorname{Pig} 1$ (6 mg dye)

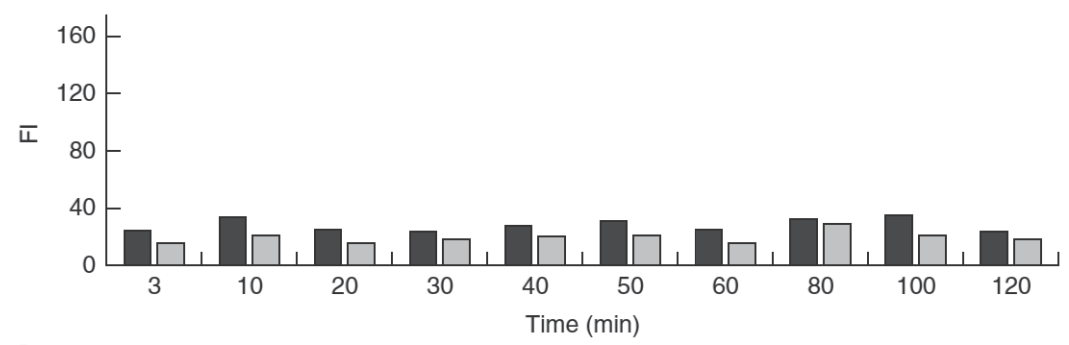

b Pig 2 (3 mg dye)

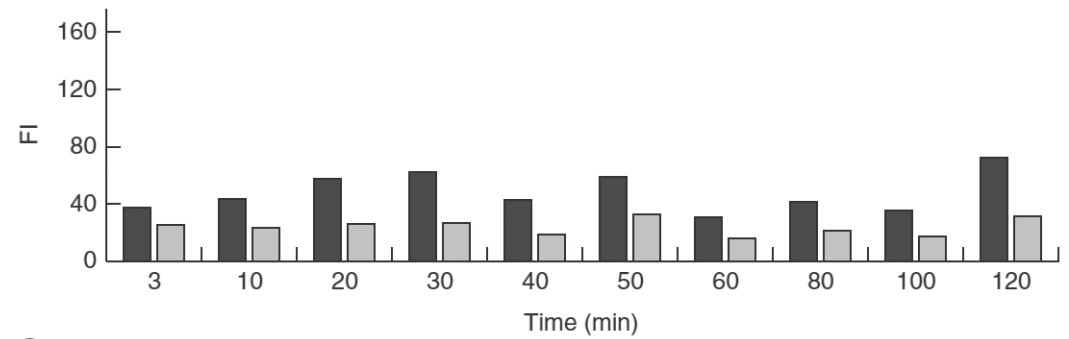

C Pig 3 (12 mg dye)

Figure 1 Absolute fluorescence intensity (FI) at various time points for the left ureter in a pig 1 (IRDye ${ }^{\circledR}$ 800-BK dose $6 \mathrm{mg}$ ), b pig 2 (dose $3 \mathrm{mg}$ ) and c pig 3 (dose $12 \mathrm{mg}$ ), showing the mean Fl for the target (ureter) and its background

Table 1 Influence of wall thickness and dye concentration on fluorescence intensity and target-to-background ratio of pig ureters

\begin{tabular}{lllll}
\hline $\begin{array}{l}\text { Ureter } \\
\text { No }\end{array}$ & $\begin{array}{l}\text { Concentration of } \\
\text { IRDye } \\
\text { PBS 800BK in }\end{array}$ & $\begin{array}{l}\text { Ureter wall } \\
\text { thickness }(\boldsymbol{\mu m})\end{array}$ & $\begin{array}{l}\text { Fluorescence } \\
\text { intensity }\end{array}$ & $\begin{array}{l}\text { Target to } \\
\text { background ratio }\end{array}$ \\
\hline 1 & $1: 4$ & 119.2 & 38 & 6.6 \\
\hdashline 2 & $1: 16$ & 129.0 & 56 & 40 \\
\hdashline 3 & $1: 64$ & 112.7 & 46 & 7.2 \\
\hline 4 & $1: 256$ & 93.3 & & 8.2 \\
\hline
\end{tabular}

PBS: phosphate-buffered saline 


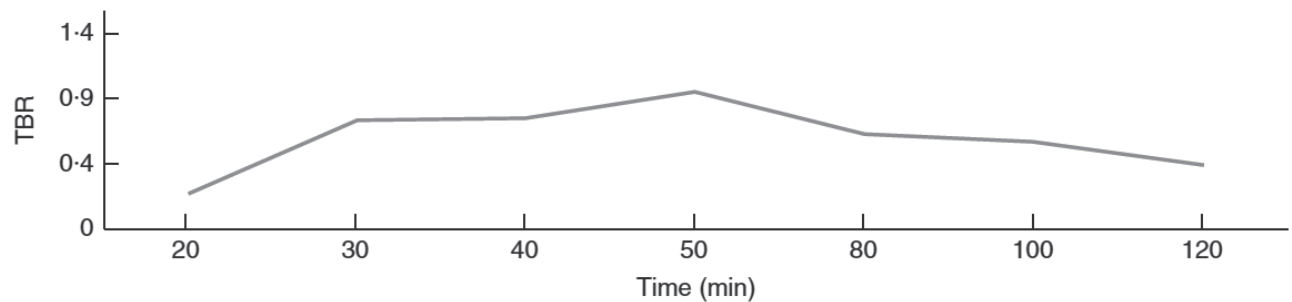

a $\operatorname{Pig} 1(6 \mathrm{mg}$ dye $)$

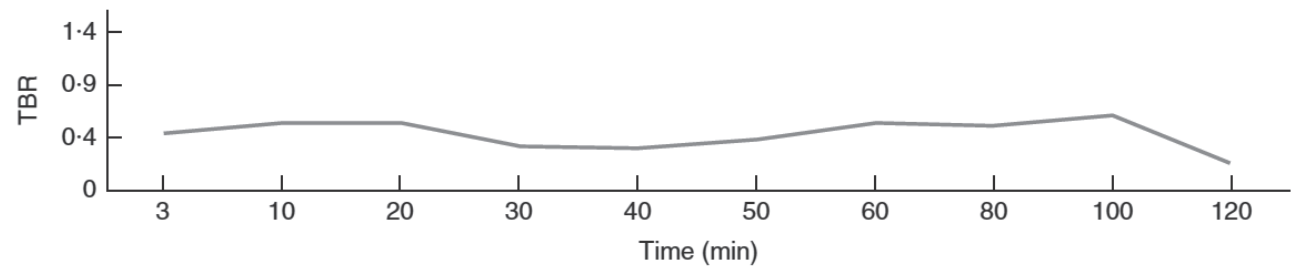

b Pig 2 (3 mg dye)

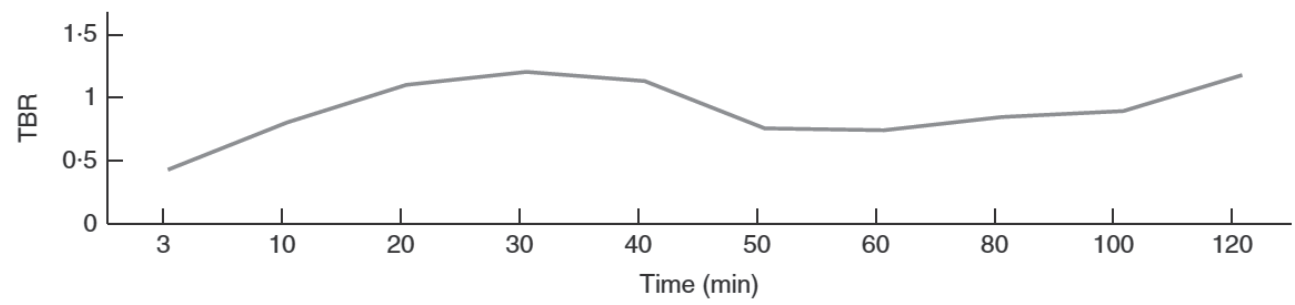

C Pig 3 (12 mg dye)

Figure 2 Target-to-background ratio (TBR) at various time points during laparoscopy for the left ureter in a pig 1 (IRDye ${ }^{\oplus} 800$-BK dose $6 \mathrm{mg}$ ), b pig 2 (dose $3 \mathrm{mg}$ ) and c pig 3 (dose $12 \mathrm{mg}$ )
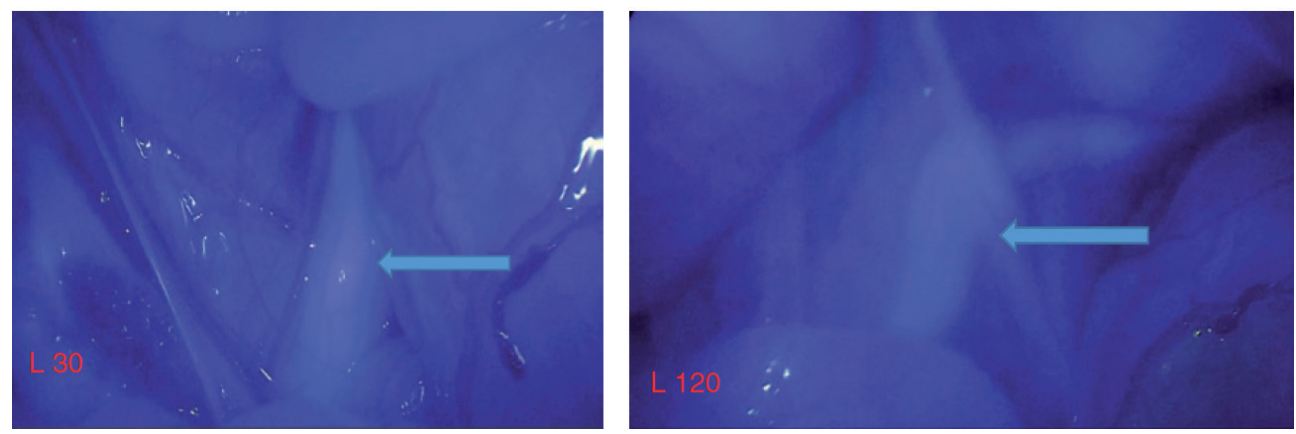

Figure 3 Screenshots of left ureter after $30 \mathrm{~min}$ (right) and $120 \mathrm{~min}$ (left) after injection of $6 \mathrm{mg} \mathrm{IRDye}{ }^{\circledast} 800$-BK in pig 1. Arrow indicates the ureter. 


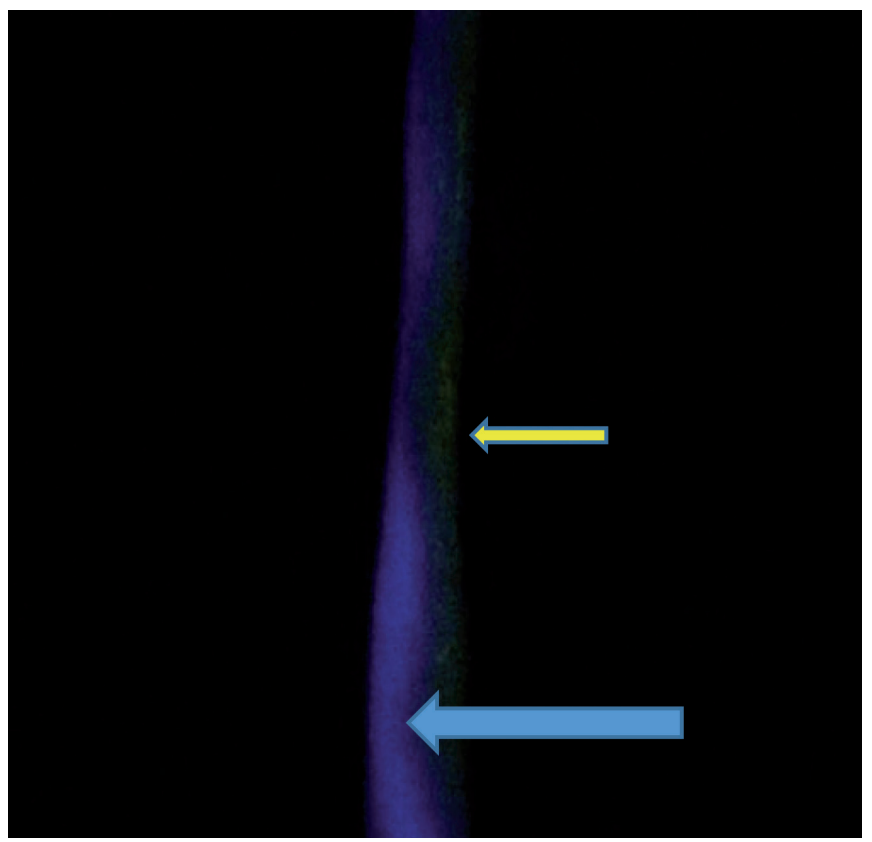

The blue arrow indicates the ureter, and the yellow arrow the overlying fatty tissue

Figure 4 Screenshot of explanted pig ureter 2
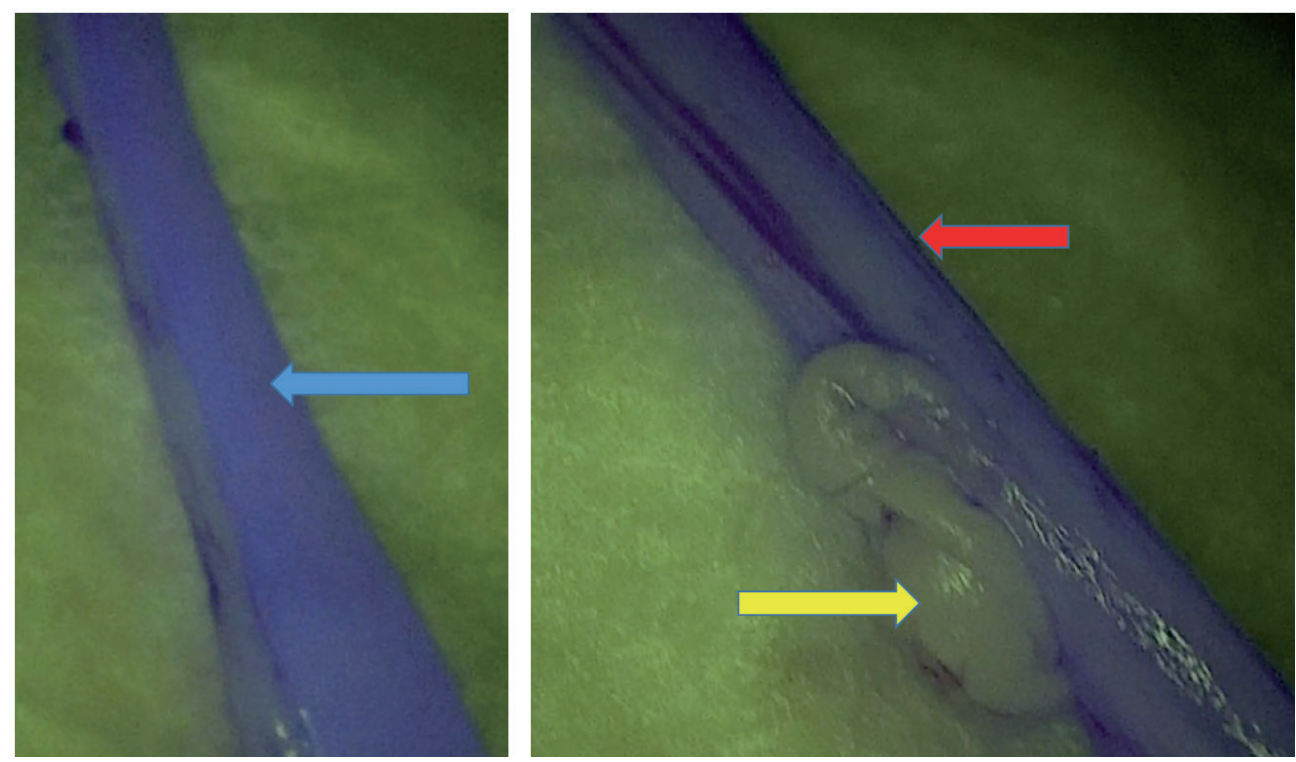

Right: inner surface of the ureter. Left outer layer of the same ureter. The blue arrow indicates the luminal surface of the ureter, the red arrow the outer layer of the ureter, and the yellow arrow the overlying fatty tissue.

Figure 5 Screenshots of explanted pig ureter 1 
Table 2 Influence of wall thickness and concentration of dye on fluorescence intensity and target-to background ratio of human ureters

\begin{tabular}{|c|c|c|c|c|}
\hline $\begin{array}{l}\text { Ureter } \\
\text { No }\end{array}$ & $\begin{array}{l}\text { Concentration of } \\
\text { IRDye }{ }^{\oplus} 800 \text { BK in }^{-} \\
\text {PBS }\end{array}$ & $\begin{array}{l}\text { Ureter wall } \\
\text { thickness }(\mu \mathrm{m})\end{array}$ & $\begin{array}{l}\text { Fluorescence } \\
\text { intensity }\end{array}$ & $\begin{array}{l}\text { Target to } \\
\text { background ratio }\end{array}$ \\
\hline 1 & $1: 1$ & 150.8 & 42 & 7.4 \\
\hline 2 & $1: 4$ & 171.6 & 49 & 8.8 \\
\hline 3 & $1: 16$ & 158.9 & 37 & 6.4 \\
\hline 4 & $1: 64$ & 167.8 & 45 & 8.0 \\
\hline 5 & $1: 256$ & 189.9 & 25 & 4.0 \\
\hline 6 & $1: 1024$ & 133.8 & 22 & 3.4 \\
\hline 7 & 1:4096 & 162.1 & 11 & 1.2 \\
\hline
\end{tabular}

PBS: phosphate-buffered saline

\section{Ex vivo fluorescence imaging of human ureters}

Wall thickness of seven post-mortem human ureters ranged from 133.8 to 189.0 (mean 162) $\mu \mathrm{m}$. Clear delineation of all seven ureters was possible using fluorescence imaging, at all dye concentrations, with $\mathrm{Fl}$ and TBR ranging from 11 to 49 and 1.20 to 8.80 respectively (Table 2). Again, areas of ureter covered by a layer of fatty tissue did not show fluorescence.

\section{DISCUSSION}

IRDye ${ }^{\circledast} 800-B K$ has been developed specifically for intra- operative NIRF visualization of the ureters. The goals of the present experiments were to see whether the new dye would be suitable for visualization of the ureter and to perform a first preliminary evaluation of factors likely to influence the signal produced.

The study confirmed visualization of the ureters in real time both in vivo and ex vivo. In vivo, administration of a bolus of 6 and $12 \mathrm{mg}(0.15$ and $0.30 \mathrm{mg} / \mathrm{kg}$ respectively) allowed excellent and clear visualization of the ureters in NIRF mode. The dose of $3 \mathrm{mg}(0.08 \mathrm{mg} / \mathrm{kg})$ also provided enhanced imaging of the ureter, although the FI was weak.

The maximum intensity of the fluorescence signal was seen during contractions of the ureter, showing the transport of intraluminal urine from proximal to distal. The relationship with peristalsis is a slight drawback, as the signal is not present permanently, although this will be the case for every signal produced by a dye transported in urine. This seems greatly surpassed by the excellent signal during these episodes of contractions.

A signal was observed for up to 120 min after the administration of dye, when the surgical procedures were terminated. A study with longer continuous NIRF visualization of the ureter may be beneficial.

Each ureter showed varying $\mathrm{FI}$ and TBR throughout the in vivo study. A possible explanation may have been failure to standardize the laparoscope-target distance. It is 
possible that the laparoscope was at different distances from the ureter, resulting in different amounts of fluorescence being detected and influencing the TBR. Kono and colleagues ${ }^{19}$ reported that the signal contrast on fluorescence images of bile duct samples differed significantly between the laparoscopic imaging systems, and tended to decrease as the laparoscope-target distance increased and porcine tissues covering the samples became thicker.

In the in vivo study, all concentrations of dye tested resulted in enhanced visualization of the ureter compared with conventional white light, as indicated by a positive TBR value. Doses of 6 and $12 \mathrm{mg}$ gave the best intraoperative results. Doses that resulted in a subjectively better signal and a higher measured absolute intensity did not always result in a higher TBR. This may be explained by the influence of the dose both on target and background signals. A low Fl of the target may be accompanied by a very low Fl of the background, whereas a higher $\mathrm{Fl}$ of the target may be accompanied by a high background signal.

At all doses studied it was observed that urine flow in the ureter was not continuous but related to contractions, with the fluorescent signal related to urine flow, resulting in fluctuations in the fluorescence signal detected. As a result, the observed and measured signals and TBRs may vary over time during in vivo studies. In contrast, in the ex vivo experiments a uniform background could be chosen, which enabled comparison of the TBR between experiments.

To further study the dose-visibility relationship, explanted pig ureters and dye dilutions ranging from 1:4 to 1:256 were used. A clear delineation of the ureters was obtained at all doses, with a TBR ranging from 6.6 to 10.20. As it has been suggested previously that ureter wall thickness may have a negative influence on NIRF intensity ${ }^{12}$, and that findings in animal studies may not be representative of humans, human ureters with their thicker walls were also subjected to ex vivo NIRF imaging. Dye concentrations from 1:1 up to 1:4096 showed successful NIRF imaging in all human ureters, with TBRs ranging from 1.20 to 8.80 . The increased wall thickness therefore did not prevent the fluorescence signal, although a decrease in FI was observed at the lowest concentrations. In both the porcine and human ex vivo experiments, not all results for TBR had a logical explanation, but it seems that the concentration of dye had a greater influence than thickness of the ureter wall.

No complications or adverse reactions attributable to the dye were observed during any operations. Only a slight and transient decrease in intraoperative peripheral oxygen saturation was noted during the first minute after injection of the fluorescent dyes.

A limitation of the ex vivo experiments is that two influences were studied at the same time: dye concentration and wall thickness. Ideally, different concentrations of dye should be tested in the same ureters, and the same con- centration in different ureters. The first of these types of experiment is not feasible, because the fluorescent signal was retained in the ureter wall. Such an experiment can therefore be performed only using artificial material. The second experiment has not yet been undertaken. 
Despite the promising results, the present findings must be interpreted with caution. Owing to the limited availability of pigs and ureters, it was not possible to study enough ureters for solid statistical conclusions to be drawn. Another limitation is that concentrations of dye in the urine were not measured. This requires availability of the structural formula of the dye and a method for such measurement. These were not available to the authors in this early phase of development and application of the dye.

It was disappointing in the ex vivo study that even the smallest layer of fat covering the ureter prevented a decent signal from being obtained. It is known that penetration of the NIRF signal is limited to approximately $10 \mathrm{~mm} .{ }^{20} \mathrm{NIRF}$ imaging with a stronger dye or with optimized equipment was hoped to enhance such penetration. The present experiment illustrates that the signal may be improved with use of a better dye, but that this does not affect depth penetration per se. In the in vivo study, nevertheless, the porcine ureters could be identified clearly without any manipulation or dissection of the overlying tissues. This suggests that IRDye ${ }^{\circledast} 800 \mathrm{BK}$ has the potential to detect the ureter in spite of the overlying peritoneum. Future studies should evaluate the maximum depth of penetration of the NIRF signal and the clinical value of this dye in human subjects.

This novel dye enables visualization of the ureters. NIRF imaging with this dye seems a valuable addition to conventional white light laparoscopy. Further studies are needed to see if it can become a worthwhile addition to improve clinical practice. 


\section{REFERENCES}

1. Parpala-Spårman T, Paananen I, Santala M, Ohtonen P, Hellström P. Increasing numbers of ureteric injuries after the introduction of laparoscopic surgery. Scand J Urol Nephrol 2008; 42: $422-427$.

2. Park JH, Park JW, Song K, Jo MK. Ureteral injury in gynecologic surgery: a 5-year review in a community hospital. Korean J Urol 2012; 53: 120-125.

3. Palaniappa NC, Telem DA, Ranasinghe NE, Divino CM. Incidence of iatrogenic ureteral injury after laparoscopic colectomy. Arch Surg 2012; 147: 267-271.

4. DaSilva G, Boutros M, Wexner SD. Role of prophylactic ureteric stents in colorectal surgery. Asian J Endosc Surg 2012; 5: 105-110.

5. Assimos DG, Patterson LC, Taylor CL. Changing incidence and etiology of iatrogenic ureteral injuries. J Urol 1994; 152: 2240 - 2246.

6. Zafar SN, Ahaghotu CA, Libuit L, Ortega G, Coleman PW, Cornwell EE III et al. Ureteral injury after laparoscopic versus open colectomy. JSLS 2014; 18: pii: e2014.00158.

7. Namikawa T, Sato T, Hanazaki K. Recent advances in near-infrared fluorescence-guided imaging surgery using indocyanine green. Surg Today 2015; 45: 1467-1474.

8. Zhu B, Sevick-Muraca EM. A review of performance of near-infrared fluorescence imaging devices used in clinical studies. Br J Radiol 2015; 88: 20140547.

9. Siddighi S, Yune JJ, Hardesty J. Indocyanine green for intraoperative localization of ureter. Am J Obstet Gynecol 2014; 211: 436.e1 - 436.e2.

10. Matsui A, Tanaka E, Choi HS, Kianzad V, Gioux S, Lomnes SJ et al. Real-time, near-infrared, fluorescence-guided identification of the ureters using methylene blue. Surgery 2010; 148: 78-86.

11. Polom W, Markuszewski M, Rho YS, Matuszewski M.Use of invisible near infrared light fluorescence with indocyanine green and methylene blue in urology. Part 2. Cent European J Urol 2014; 67: 310-313.

12. Al-Taher M, van den Bos J, Schols RM, Bouvy ND, Stassen LP. Fluorescence ureteral visualization in human laparoscopic colorectal surgery using methylene blue. J Laparoendosc Adv Surg Tech A 2016; 26: 870-875.

13. Schols RM, Lodewick TM, Bouvy ND, van Dam GM, Dejong CH, Stassen LP. Application of a new dye for near-infrared fluorescence laparoscopy of the ureters: demonstration in a pig model. Dis Colon Rectum 2014; 57: 407 - 411.

14. Korb ML, Huh WK, Boone JD, Warram JM, Chung TK, de Boer E et al. Laparoscopic fluorescent visualization of the ureter with intravenous IRDye800CW. J Minim Invasive Gynecol 2015; 22: 799-806.

15. Tanaka E, Ohnishi S, Laurence RG, Choi HS, Humblet V, Frangioni JV. Real-time intraoperative ureteral guidance using invisible near-infrared fluorescence. J Urol 2007; 178: 2197 - 2202.

16. NC3Rs Reporting Guidelines Working Group. Animal research: reporting in vivo experiments: the ARRIVE guidelines. JPhysiol 2010;588:2519-2521.

17. Sawkar HP, Kim DY, Thum DJ, Zhao L, Cashy J, Bjurlin M et al. Frequency of lower urinary tract injury after gastrointestinal surgery in the nationwide inpatient sample database. AmSurg 2014;80:1216-1221.

18. Schols RM, Bouvy ND, Masclee AA, vanDam RM, Dejong CH, Stassen LP. Fluorescence cholangiography during laparoscopic cholecystectomy: a feasibility study on early biliary tract delineation. Surg Endosc 2013;27:1530-1536.

19. Kono Y, Ishizawa T, Tani K, Harada N, Kaneko J, Saiura A et al. Techniques of fluorescence cholangiography during laparoscopic cholecystectomy for better delineation of the bile duct anatomy. Medicine (Baltimore) 2015; 94: e1005.

20. Vahrmeijer AL, Hutteman M, van der Vorst JR, van de Velde CJ, Frangioni JV. Image-guided cancer surgery using near-infrared fluorescence. Nat Rev Clin Oncol 2013; 10: 507-518. 
PART III 
NEAR INFRARED FLUORESCENCE IMAGING IN THYROID SURGERY 


$$
-\frac{1}{1}
$$




\section{Feasibility of the use of NIRF in parathyroid identification}

Jacqueline van den Bos, Lottie van Kooten, Sanne M.E. Engelen, Tim Lubbers, Laurents P.S. Stassen, Nicole D. Bouvy.

Head and Neck 2019 41(2):340-348 


\section{ABSTRACT}

\section{Background}

This study assessed the feasibility of near-infrared fluorescence imaging with indocyanine green (ICG) to identify the parathyroid glands (PGs) intraoperatively and to assess their perfusion after thyroid resection.

\section{Methods}

Patients undergoing elective thyroidectomy were enrolled in this prospective study. An intravenous bolus of $7.5 \mathrm{mg}$ ICG was administered twice: the first bolus to identify the PGs before resection of the thyroid and the second to assess vascularization of the PGs after resection.

\section{Results}

A total of 30 operations in 26 patients were included. In 17 surgeries (56.7\%), fluorescence imaging was of added value, especially to confirm the presence of a suspected PG. No intraoperative or postoperative complications occurred because of the use of ICG.

\section{Conclusion}

Near-infrared fluorescence imaging with the use of ICG for intraoperative identification of the PGs and the assessment of its vascularization is feasible and safe and can provide more certainty about the location of the PGs. 


\section{INTRODUCTION}

latrogenic injury of the parathyroid glands (PGs) is the most common complication after total thyroidectomy.' Damage of the PGs can cause hypoparathyroidism, which results in hypocalcemia. The reported incidence of hypocalcemia after total thyroidectomy varies widely: between $1.6 \%$ and $50 \% .^{2-4}$ Hypocalcemia can result in increased morbidity, including cardiac arrhythmias and tetany leading to pro-longed hospitalization and even death. ${ }^{5}$ To avoid parathyroid injury, it is essential to identify the PGs and preserve their blood supply. However, this can be challenging due to their small size and the fact that they can be difficult to distinguish from their surrounding tissues. Intraoperative guidance to help identify and assess the PGs during thyroid surgery may prevent surgical damage and thus provide a better post-operative outcome and quality of life. A few intraoperative imaging techniques have been described. Dudley ${ }^{6}$ was the first to use intravenous administration of methylene blue (MB) to stain parathyroid tissue in vivo in 1971. After publication of this study, MB has been implemented by other surgeons. ${ }^{7}$ The chance of complications due to intravenous administration of $\mathrm{MB}$, including neurotoxicity and acute $\mathrm{MB}$-induced phototoxicity, pain at infusion site and nausea is a major drawback of thistechnique. ${ }^{7-10}$ To avoid these complications due to a high dose of $\mathrm{MB}$, a lower dose in combination with near-infrared fluorescence (NIRF) imaging was investigated.11However,in analogy with the use of MB for staining without NIRF-imaging, this technique mainly visualizes abnormal PGs. To avoid iatrogenic damage, visualization of normal PGs is essential. A technique in which both abnormal and normal PGs can be identified is fluorescence imaging with 5-aminolevulinicacid. ${ }^{12}$ However, this technique also has its side effects and requires extensive photosensitization preparation and thereby, patients have to be shielded from direct light exposure for 48 hours to prevent phototoxic reactions. ${ }^{13} \mathrm{~A}$ promising technique to visualize healthy and diseased parathyroid tissue has emerged in the use of intraoperative NIRF imaging using intravenously administered indocyanine green (ICG). This safe and rapidly evolving intraoperative imaging modality is described for use in a wide range of surgical procedures, such as identification of the biliary anatomy, assessment of anastomotic perfusion, and sentinel lymph node mapping. ${ }^{14-16}$ Suh et al. showed fluorescent PGs when using NIRF imaging with ICG in three dogs. ${ }^{17}$ Other studies showed the possibility of ICG fluorescent angiography to assess the remaining blood supply to the PGs in humans. ${ }^{18-20}$ Thus, NIRF imaging with ICG seems to be a promising technique to identify PGs during thyroid surgery and assess remaining perfusion of the PGs after thyroid removal. The aim of this study was to assess the feasibility of NIRF imaging with ICG to identify PGs during thyroid surgery and to get an impression of the vascularization of the preserved PGs. 


\section{METHODS}

This study was conducted at the Department of Surgery of the Maastricht University Medical Center (MUMC, Maastricht, Netherlands) between March 2017 and October 2017.The Medical Ethical Committee approved of this study, the study was conducted in full accordance with the ethical principles in the Declaration of Helsinki $2014^{21}$ and the protocol was registered on Clinicaltrials.gov with registration number NCT03012438. Consecutive men and women patients, aged 18 years and older, scheduled for elective total, hemithyroidectomy or completion thyroidectomy, with a normal liver and renal function and with written informed consent were eligible for inclusion. Subjects were excluded in case of known ICG, iodine, penicillin or sulfa-hypersensitivity; pregnancy or breastfeeding; intravenous heparin injection in the last 24 hours (all contraindications for the use of intravenous ICG). A flowchart of the study procedures is shown in Figure 1.

\section{Laparoscopic fluorescence imaging system and ICG administration}

A commercially available laparoscopic fluorescence imaging system (Karl Storz GmbH \& CO., Tuttlingen, Germany), including a plasma light guide, a 30-degree $10 \mathrm{~mm}$ laparoscope applicable for near infrared (NIR) light was used. Observations were done using white light, from the regular operation room-lights and without camera, and in NIRF mode, using the dedicated system. Video recordings were made using the laparoscopic system. ICG is a fluorescent contrast dye, which binds to plasma proteins and becomes fluorescent once excited by NIR light between about 820 and $840 \mathrm{~nm}$. The dye was diluted with sterile water to a concentration of $2.5 \mathrm{mg} / \mathrm{mL}$. Although having the laparoscopic imaging system in NIRF modus pointed at the thyroid, a bolus of $7.5 \mathrm{mg}(3 \mathrm{~mL}$ ) ICG (VERDYE, Diagnostic Green $\mathrm{GmbH}$, Aschheim-Dornacht, Germany) was administered intravenously at two different time points. The aim of the first bolus was to identify the PGs before removal of the (hemi-) thyroid. The second bolus was used to assess vascularization of the PGs directly after thyroid resection. Both boluses of ICG were given just before using the NIRF-modus of the fluorescence system. In all total thyroidectomies, the surgeons searched for four PGs. In all hemithyroidectomies and completing thyroidectomies the surgeons searched for two PGs.

\section{Data collection}

The following intraoperative parameters were registered: whether PGs were identified, subjective fluorescence intensity of the PGs according to the surgeon, opinion of the surgeon about the usefulness of the technique, occurrence of intraoperative complications, the amount of time used for fluorescence imaging, and total surgical time.

The fluorescence intensity was scored by the performing surgeon on a 1-3 grading scale: 1 means that the PG is black after injection of ICG, no fluorescence visible, 2 means that the PG is fluorescent, but no more than the surrounding tissue, and 3 if the PG is more fluorescent 


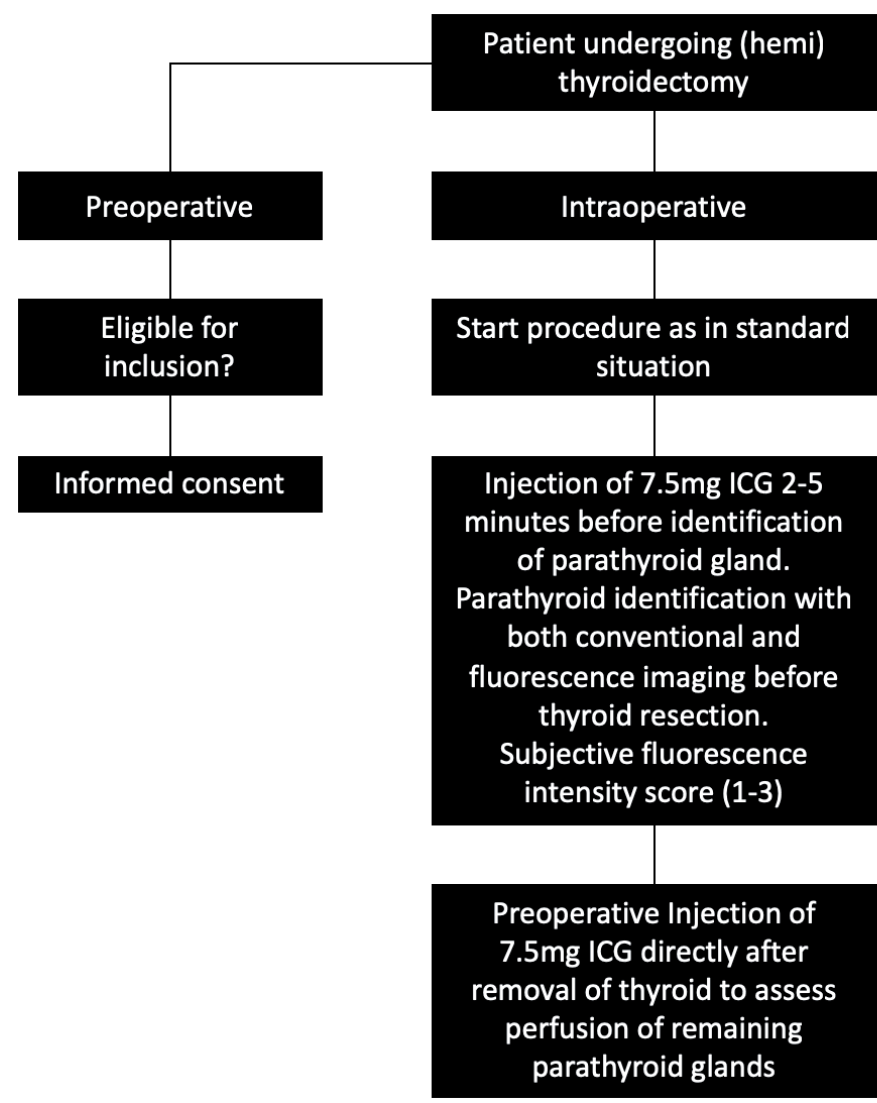

Figure 1 Flowchart of the study procedures

than the surrounding tissue. Postoperatively, the thyroid specimen was checked by the pathologist for the presence of parathyroid tissue in the thyroid specimen. Perioperative complications were registered and fluorescence intensity on the video images was analyzed. The fluorescence intensity was analyzed using OsiriX Lite V8.5.2 Imaging software (Pixmeo, Geneva, Switzerland) to determine the target-to-background ratio (TBR). The TBR was defined as the mean fluorescence intensity ( $\mathrm{FI})$ of three points region of interest (ROIs) in the target (i.e., PGs) minus the mean FI of three ROls in the back-ground (BG) (i.e., surrounding tissue) divided by the mean FI of the BG ROIs. In formula TBR = (fluorescence intensity of target-fluorescence intensity of background)/fluorescence intensity of background. ${ }^{22}$ When this score is zero, there is no difference between the fluorescence intensity of the PG and the surrounding tissue. A TBR above zero indicates a more fluorescent PG compared to surroundings, and a TBR below zero indicates that the surroundings are more fluorescent compared to the PG. 


\section{Statistical analysis}

Statistical analysis was performed using the Statistical Package for the Social Sciences v23 (IBM, Amonk, NY). A descriptive analysis was conducted for the patients' baseline characteristics and the following outcome measurements: total operation time, extra operation time, the presence of PGs in the resected thyroid, number of PGs identified in white light and NIRF, occurrence of perioperative complications, and the usefulness of the technique. The independent sample-test was used to assess whether there was a relation between the fluorescence intensity and factors, such as malignant or benign disease and normal or low calcium.

\section{RESULTS}

A total of 30 surgeries (hemi-, total or completion thyroidectomy) in 26 patients were included. The majority of participants were female (73\%) and the most common indication for thyroid surgery was suspected thyroid cancer (56.7\%). Median body mass index (BMI) was $25.21 \mathrm{~kg} / \mathrm{m}^{2}$. Further patient characteristics are shown in Table 1.

\section{Feasibility}

No perioperative or postoperative complications due to the use of ICG occurred. Complications that occurred were an arterial bleeding after surgery for which reoperation was needed and a postoperative wound infection that required antibiotic treatment in another patient. The mean surgical time was 92 minutes ( \pm 32 minutes) for all surgeries. Herein, on average 5 minutes and 35 seconds ( \pm 94 seconds) were used for NIRF imaging. For a total thyroidectomy, mean surgical time was 126 minutes ( \pm 54 minutes); herein, 6 minutes and 27 seconds ( \pm 95 seconds) were spent using NIRF. A hemithyroidectomy took on average 83 minutes ( \pm 17 minutes) of which 5 minutes and 29 seconds ( \pm 55 seconds) were used for NIRF imaging. A completing thyroidectomy took on average 92 minutes ( \pm 22 minutes), 4 minutes and 51 seconds ( \pm 55 seconds) were spent using NIRF imaging.

\section{Subjective usefulness of the technique}

In 17 patients (57\%), fluorescence imaging was rated as useful by the performing surgeon. In these patients, NIRF imaging provided certainty on both the localization and the preserved vascularization of the PGs. In two of the 17 patients, without the use of ICG, the surgical team would not have succeeded to identify a PG. In the remaining 15 patients, NIRF imaging was mainly helpful to confirm the suspicion of parathyroid tissue. An example of the obtained images is given in Figure 2 and an overview of reasons for usefulness or a lack of usefulness is given in Table 2. Three reasons were given for a lack of additional value in the remaining 13 patients. In five patients, the PGs were already clearly visualized in white light, no extra 
Table 1 Demographic and clinical details of included patients

\begin{tabular}{|c|c|}
\hline & Patients $(n=30) *$ \\
\hline Age (years) & $56.3 \pm 16$ \\
\hline Weight (kg) & $78.9 \pm 17.6$ \\
\hline BMI $\left(\mathrm{kg} / \mathrm{m}^{2}\right)$ & $28.3 \pm 6.9$ \\
\hline \multicolumn{2}{|l|}{ Gender } \\
\hline Male & $8(26.7)$ \\
\hline Female & $22(73.3)$ \\
\hline \multicolumn{2}{|l|}{ ASA-classification } \\
\hline 1 & $3(10)$ \\
\hline 2 & $25(83.3)$ \\
\hline 3 & $2(6.7)$ \\
\hline \multicolumn{2}{|l|}{ Type of surgery } \\
\hline Total thyroidectomy & $6(20)$ \\
\hline Completion thyroidectomy & $6(20)$ \\
\hline Hemi-thyroidectomy & $18(60)$ \\
\hline \multicolumn{2}{|l|}{ Indication for surgery } \\
\hline Multinodular goiter & $5(16.7)$ \\
\hline Graves' disease & $1(3.3)$ \\
\hline Suspected thyroid cancer & $17(56.7)$ \\
\hline Proven thyroid cancer & $7(23.3)$ \\
\hline \multicolumn{2}{|l|}{ Histology thyroid specimen } \\
\hline Multinodular goiter & $10(33.3)$ \\
\hline Thyroid cancer & $11(36.7)$ \\
\hline Normal thyroid & $3(10)$ \\
\hline Follicular adenoma & $5(16.7)$ \\
\hline Thyroiditis & $1(3.3)$ \\
\hline
\end{tabular}

*The 4 patients with two included surgeries are counted twice.

Data are presented as mean \pm SD or number (\%).

SD: Standard deviation

certainty was needed for these patients. A second reason given for a lack of added value was that the PGs remained black after ICG administration in three patients. However, in these patients, the surgeon notes that itis possible that the PG was already dissected $(n=1$ in which a PG was found in the histology assessment), or the thyroid was already manipulated extensively before using NIRF, possibly causing damage of the blood supply toward the PG ( $n=2$ hemithyroidectomies in which no calcium levels were determined due to the expected remaining two PGs in the other half thyroid). Therefore, although not for localization, the found black PG in these patients could have been of added value to indicate the surgeon that auto transplantation of this PG should be considered. The third reason surgeons gave for a lack of added value was in two patients of lymphatic metastatic disease and two patients of non-metastatic malignant cases: "the well-vascularized cancerous tissue seemed to show misleading fluorescence intensity." Because this tissue was so well vascularized, a lot 
of background fluorescence was present in these patients, making it difficult to distinguish between the cancerous tissue and the PGs based on NIRF imaging. Further perioperative data are listed in Table 3.

\section{Objective imaging and PG identification}

In all total thyroidectomies and hemithyroidectomies, at least one PG was seen in either white or NIRF light before thyroid resection. In total, 41 PGs were visualized in white light in 25 patients, whereas with NIRF imaging in total 31 PGs could be identified in 23 patients. In three patients, no PG was identified during thyroid resection. All three patients underwent a completion thyroidectomy. In two hemithyroidectomy patients in whom initially no PG could be identified in white light, one PG was seen after the use of NIRF. In one patient, only one PG was seen in white light, whereas two were seen in NIRF light. These three patients underwent a hemithyroidectomy and no PGs were found in the resected material. In nine patients, more PGs were visualized in white light. This group consisted of three total thyroidectomies and six hemithyroidectomies. In three of these patients, NIRF was used after extensive manipulation of the thyroid. In 18 patients, the same number of PGs was seen in white light as in NIRF light. In three patients, the number of PGs seen in white light is higher than expected, indicating a possible wrong identification of a PG. In NIRF imaging, a lower number of PGs were seen in two of these patients.

\section{Histology}

In seven patients, parathyroid tissue was found in the histopathology specimen. As can be seen in Table 4, in all these patients, only one PG was found in the specimen. Three out of these seven patients underwent a total thyroidectomy. In one of these patients, three PGs were seen both in white and NIRF light; in another patient, three PGs were seen in white light and one in NIRF; and in the third patient, two PGs were seen in white light and no PGs were seen with NIRF imaging. Another patient underwent a completion thyroidectomy in whom two PGs were seen in both white and NIRF light. The remaining three patients underwent a hemithyroidectomy. In all of them, two PGs were identified in white light; in one of those patients, no PG was seen with NIRF; and in the other two, only one PG was seen with NIRF imaging. Because in these patients parathyroid tissue was found in the resected thyroid, it is possible that what the surgeon called parathyroid tissue in white light actually was other tissue. No significant correlation was found between the existence of parathyroid tissue in the histology specimen and the subjective fluorescence intensity during surgery.

\section{Fluorescence intensity}

Mean subjective fluorescence before and after thyroid re-section was $2.53( \pm 0.6)$ and 2.37 $( \pm 0.7)$, respectively. Mean TBR before and after thyroid resection was $4.2( \pm 7.6)$ and $2.5( \pm 4.7)$, respectively. The subjective score on a scale 1-3 was compared with the measured TBR. In 


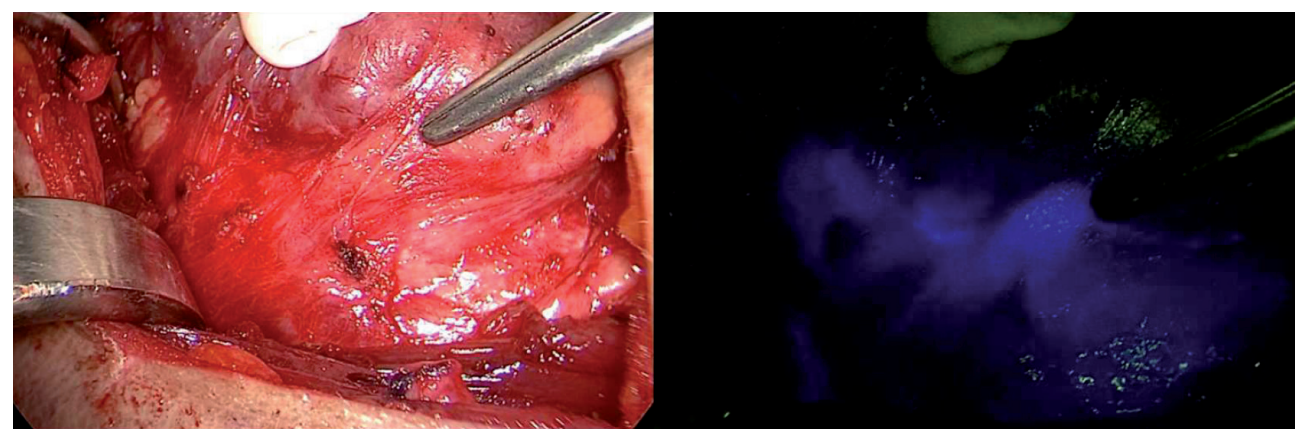

Left: the suspected PG indicated by tweezers in white light.

Right: the suspected PG illuminated bright blue in fluorescence light. The NIRF signal outside the parathyroid gland is caused by the blood vessels towards and from the parathyroid gland.

Figure 2 Example of obtained images

two PGs, a subjective score of 1 (meaning PG is darker com-pared to surrounding) was given before thyroidectomy. In these patients, a mean TBR of $0.8( \pm 1.4)$ was found for those PGs, compared to a mean TBR of $0.7( \pm 0.3)$ in the PGs with a score of 2 (meaning PG as fluorescent as sur-rounding) and a mean TBR of $6.0( \pm 8.9)$ in the PGs in which a score of three was given (PG more fluorescent com-pared to surrounding). After thyroid resection in two patients, a score 1 was given, with a mean TBR of $0.2( \pm 0.2)$, a score of 2 resulted in a mean TBR of 2.4 $( \pm 6.1)$ and a subjective score of 3 in a TBR of $2.8( \pm 4.1)$. In none of the PGs which was scored as 3, a TBR below zero was measured. A higher TBR of the PGs before thyroid re-section was seen in patients with benign pathology com-pared to patients with a malignancy $(P=0.044$; $\mathrm{Cl}=-9.6$ to-0.14). This fluorescence intensity was on average $6.01( \pm 2.2)$ for benign histology and $1.1( \pm 0.8)$ in patients with malignant disease of the thyroid. The TBR after thyroid removal was with $3.0( \pm 5.1)$ and $1.8( \pm 4.0)$, respectively, not significantly different in patients with benign histology compared to malignant disease. No other factors contributing to a significant higher or lower TBR or subjective fluorescence intensity were found.

\section{Function}

In all patients who underwent total thyroidectomy, calcium supplementation was started postoperatively to prevent hypocalcemia, according to standard care practice. Normal calcium levels were found in all patients 6 hours after surgery. In three patients who underwent total thyroidectomy, calcium ion levels dropped temporarily below the normal calcium-ion value of our laboratory (normal range $=1.1-1.3 \mathrm{mmol} / \mathrm{L}$ ) the day after surgery. Calcium ion levels of these patients were 1.02, 1.03, and $1.09 \mathrm{mmol} / \mathrm{L}$, respectively. Measured TBR in these patients after thyroid resection was lower than average with a mean TBR of 0.6 $( \pm 0.7)$, compared to a mean TBR of $5.1( \pm 2.4)$ in patients with a normal calcium ion level the day after surgery $(P=0.12)$. Calcium levels in these patients were restored after 2 weeks to $2.16,2.30$, and $2.32 \mathrm{mmol} / \mathrm{L}$, respectively. 


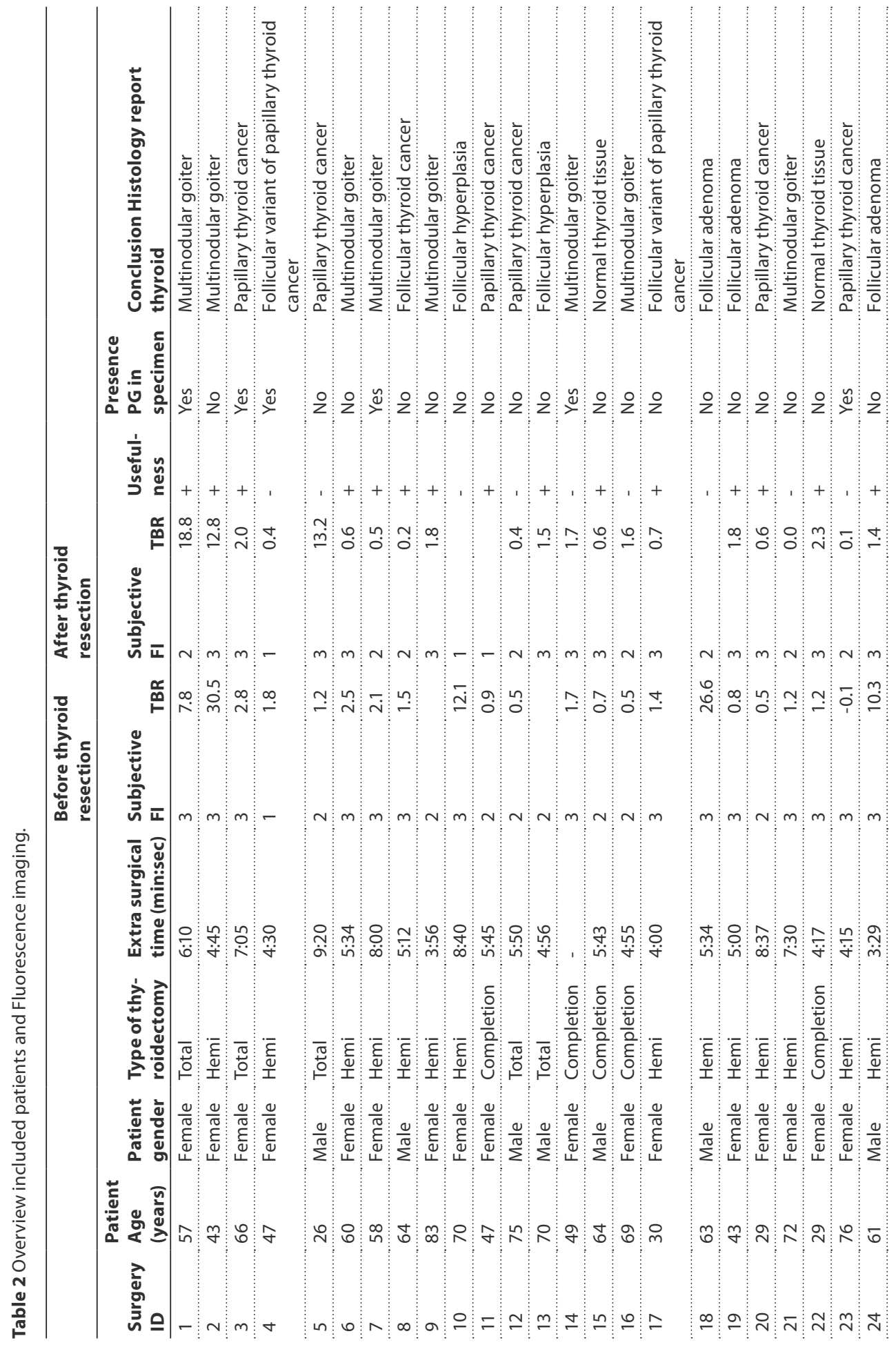




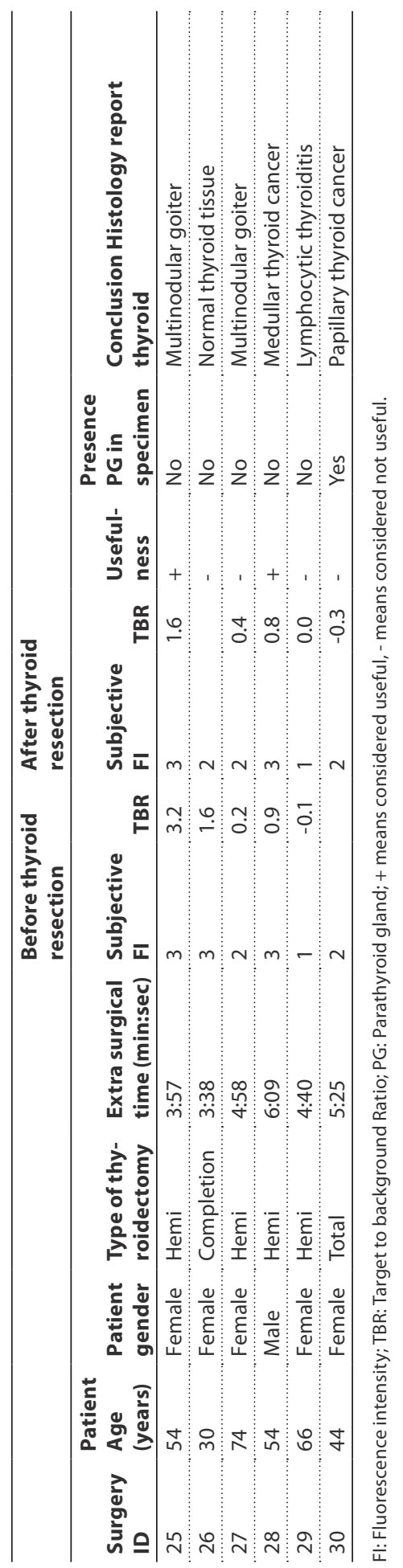


Table 3 Usefulness of the technique

\begin{tabular}{ll}
\hline NIRF imaging considered useful: & Total $\mathbf{1 7}$ cases \\
\hline Reasons: & No. of cases \\
PG was only identified using NIRF & 2 \\
Confirmation of suspected location PG & 15 \\
\hline NIRF imaging considered not useful: & Total 13 cases \\
\hline Reasons: & No. of cases \\
PGs already clearly visualized in white light, no extra certainty & \\
needed & 6 \\
PGs remained black after ICG administration & 3 \\
Surroundings too fluorescent to distinguish PGs & 4 \\
\hline
\end{tabular}

Table 4 Number of identified parathyroid glands in both white and NIRF light

\begin{tabular}{|c|c|c|c|c|c|}
\hline $\begin{array}{l}\text { Surgery } \\
\text { ID }\end{array}$ & $\begin{array}{l}\text { Type of } \\
\text { thyroidectomy }\end{array}$ & $\begin{array}{l}\text { Number of PGs } \\
\text { to be expected }\end{array}$ & $\begin{array}{l}\text { Number of PGs } \\
\text { visible in white } \\
\text { light }\end{array}$ & $\begin{array}{l}\text { Number of } \\
\text { PGs visible in } \\
\text { NIRF }\end{array}$ & $\begin{array}{l}\text { Number of PGs } \\
\text { in resected } \\
\text { thyroid }\end{array}$ \\
\hline 1 & Total & 4 & $4^{*}$ & 1 & 1 \\
\hline 2 & Hemi & 2 & 2 & 1 & 0 \\
\hline 3 & Total & 4 & 3 & 3 & 1 \\
\hline 4 & Hemi & 2 & 2 & 0 & 1 \\
\hline 5 & Total & 4 & 2 & 1 & 0 \\
\hline 6 & Hemi & 2 & 2 & 2 & 0 \\
\hline 7 & Hemi & 2 & $2^{*}$ & 1 & 1 \\
\hline 8 & Hemi & 2 & 0 & 1 & 0 \\
\hline 9 & Hemi & 2 & 2 & 2 & 0 \\
\hline 10 & Hemi & 2 & 0 & 1 & 0 \\
\hline 11 & Completion & 2 & 0 & 0 & 0 \\
\hline 12 & Total & 4 & 1 & 1 & 0 \\
\hline 13 & Total & 4 & 1 & 1 & 0 \\
\hline 14 & Completion & 2 & $2^{*}$ & $2^{*}$ & 1 \\
\hline 15 & Completion & 2 & 1 & 1 & 0 \\
\hline 16 & Completion & 2 & 0 & 0 & 0 \\
\hline 17 & Hemi & 2 & 2 & 2 & 0 \\
\hline 18 & Hemi & 2 & 1 & 1 & 0 \\
\hline 19 & Hemi & 2 & 1 & 2 & 0 \\
\hline 20 & Hemi & 2 & 2 & 1 & 0 \\
\hline 21 & Hemi & 2 & 2 & 2 & 0 \\
\hline 22 & Completion & 4 & 1 & 1 & 0 \\
\hline 23 & Hemi & 2 & 1 & 1 & 1 \\
\hline 24 & Hemi & 2 & 1 & 1 & 0 \\
\hline 25 & Hemi & 2 & 1 & 1 & 0 \\
\hline 26 & Completion & 2 & 0 & 0 & 0 \\
\hline 27 & Hemi & 2 & 1 & 0 & 0 \\
\hline 28 & Hemi & 2 & 1 & 1 & 0 \\
\hline 29 & Hemi & 2 & 1 & 0 & 0 \\
\hline 30 & Total & 4 & 2 & 0 & 1 \\
\hline Total & & 72 & 41 & 31 & 7 \\
\hline
\end{tabular}

*The tissue called parathyroid tissue in these cases could possibly be wrongly identified as PG tissue, since parathyroid tissue is found in the resected thyroid specimen. 


\section{DISCUSSION}

The main aim of this prospective, clinical study was to assess the feasibility of NIRF imaging with ICG to intraoperatively identify the PGs and to assess the perfusion after thyroid resection. The use of NIRF imaging seems safe with no occurrence of complications due to the use of ICG in this study. The marginal extension of operation time in this study, related to the use of fluorescence imaging, will probably further decrease with growing experience. Eventually, it is expected that the total operation time will be shorter because of earlier identification of the PGs. The NIRF technique has been evaluated frequently in previous studies for several purposes, but the experience with this technique in thyroid surgery is limited. In 2014, Suh et al. ${ }^{17}$ were the first to evaluate ICG for NIRF imaging of the PGs in an animal model. In three dogs, several doses of intravenous ICG ranging from 12.5 to $100 \mu \mathrm{g} /$ $\mathrm{kg}$ were administered to illuminate the PGs. In all dogs, NIRF imaging was able to detect the PGs. The optimal dose of ICG based on these experiments was estimated to be $18.75 \mu \mathrm{g} / \mathrm{kg}$. Sound et al. ${ }^{23}$ used NIRF imaging with ICG in three patients who underwent re-operative neck surgery for primary hyperthyroidism. In these series, initially, a dose of $5 \mathrm{mg}$ ICG was given, and a second dose of $5 \mathrm{mg}$ in one patient, and $3.25 \mathrm{mg}$ in the other two patients. In all three patients, the PGs could be visualized after 2 minutes and stayed fluorescent up to 20 minutes. According to Yuet al., ${ }^{24}$ a dose of 10 mg ICG might give better PG visualization intensity and time.

In the current study, PGs could be visualized in all 6 total thyroidectomies and all 18 hemi thyroidectomies. In three of the six completion thyroidectomies, the PGs could not be detected in either NIRF or white light. This is unfortunate, as especially in these more difficult cases facilitation of PG identification is helpful. Possible, explanations are the absence of PGs in the remaining thyroids or the perfusion of these PGs might have been cut off before performing fluorescence imaging. The absence of parathyroid tissue in the resected specimen shows that PGs in these patients were not located deeper in the thyroid. Possible explanations for the inability to visualize PGs seen in white light with NIRF light are manipulation of the thyroid, causing damaged blood supply toward the PG. A second possible explanation is bright background signal due to highly vascularized malignant tissue. This is supported by the lower TBRs seen in patients with malignant histology conclusions. A third possibility is that those PGs seen in white light were not actually PGs, as parathyroid tissue was present in two patients in which more PGs were seen in white light compared to NIRF light. The anatomical location of the inferior PGs can make it hard to visualize the PGs. The PGs can be found around the crossing of the recurrent nerve and the inferior thyroid artery. As we use the NIRF angiography to identify the PGs, the nearby artery could confuse the location of the PG.

As the NIRF imaging is meant as a tool to assist the surgeon, we consider the scoring of the technique by the surgeon the most relevant. The operating surgeons were, therefore, 
asked to give a subjective score for the visibility of the PG compared to the surrounding tissue. This score ranged between 1 and 3 . On average, a higher subjective score had a higher TBR although it had a broad SD. This means that the subjective observed score does not always correspond to the measured TBR. One possible explanation is the possible difference between the moving images during surgery based on which the subjective fluorescence intensity is determined and the screenshot in which the TBR is measured. Furthermore, the subjective score might be blurred by expectations, whereas the TBR is a more objective measurement, but influenced by the chosen regions of interest and background. Also, the operating surgeons were asked whether they considered the used NIRF imaging of added value. In majority, NIRF imaging was considered of added value. In two patients, it is expected that no PGs would have been visualized without the use of ICG. In other patients, the added value consisted of reassuring the surgeon who is in doubt over the location of the PGs. Reasons for a lack of added value were mainly the surgeon who is already very certain about the location or other well-vascularized tissue such as cancerous tissue that was also very fluorescent, causing the PGs not to be more fluorescent than the surroundings.

Apart from using ICG to identify the PGs, we also investigated the possibility to assess postoperative perfusion of the PGs. Earlier studies described the use of NIRF angiography of the PGs to estimate postoperative function. ${ }^{18}$ In our study, the post-resection TBR was lower than before resection, suggesting confirmation of decreased vascularization. Lang et al. ${ }^{18}$ used NIRF for intraoperative angiography with ICG to evaluate postoperative functioning of the PGs. They included70 patients and found that a high fluorescent light intensity was even more predictive for postoperative hypocalcemia than hypocalcemia immediately after operation. Vidal Fortuny et al..$^{25}$ found that in all patients who had at least one wellvascularized PG demonstrated by ICG-angiography, the PTH levels 1 day postoperatively were normal. In our study, we did not measure postoperative PTH levels. This is only standard care in parathyroid surgery in our institute. However, we do have information on calcium levels. Postoperatively calcium supplementation was started 1 day after surgery in all patients who underwent total thyroidectomy as standard care. Blood is drawn the day after a total thyroidectomy, before calcium supplementation was started. A normal calcium-ion level 1 day postoperatively would suggest normal parathyroid function, whereas calciumion levels below normal can indicate reduced parathyroid function. In three patients, calcium levels were below normal value $(1.1 \mathrm{mmol} / \mathrm{L})$. In these patients, also TBR-values seemed lower compared to patients with normal calcium levels the day after surgery, although not statistically significant. This is in line with the study performed by Zaidi et al. ${ }^{20}$ where a possible relation between fluorescence intensity and remaining function was found. In the current study, none of the patients developed permanent hypoparathyroidism.

In the current and the other available studies, 17,20,23,25 ICG is administrated just before aiming to visualize the $P G s$, as it is meant as angiography. After intravenous administration of the ICG, it takes about 30 seconds to reach the PG and thereby illuminate the gland of 
interest. We observed that not only the PG but also the thyroid gland and, to a lesser extent, the surrounding tissue are illuminated because of their vascularization, which causes ICGstaining of the background and diminishes the contrast, both subjectively and objectively by the TBR. Previous studies have shown that ICG has the property of accumulation in pathological, hyperplastic PGs, which attributes to higher fluorescence intensity of the PG contrary to the surrounding tissues. ${ }^{26}$ It is not yet known whether this is also the case in normal PGs. Further studies are warranted with earlier administration of the ICG to evaluate its influence on contrast and accumulation in the normal PG. The present study has some limitations. As the study was designed to be a feasibility study, a small number of patients were included, of which a minority of patients underwent a total or completion thyroidectomy. Therefore, only 12 patients were actually at risk of permanent hypoparathyroidism. The calcium-ion level was measured postoperatively in the patients after a total thyroidectomy. However, a limitation of this study is that we could not histologically check the tissue we saw as parathyroid tissue in NIRF because of ethical reasons. We did, however, ask the pathologist to cut extra thin samples of the removed thyroid gland to check if parathyroid tissue was found in the extracted tissue.

In summary, this study showed that NIRF imaging with ICG for identification of the PGs during thyroid surgery is feasible. For the cases in which the ICG was helpful, it especially confirmed the suspicion of the presence of a PG as seen in white light and helped to assess its vascularization after thyroid resection. In a minority of cases, NIRF identifies more PGs than white light imaging. However, it seems that in white light more wrongly identified PGs occurred. The findings of this pilot study confirm a possible role for ICG fluorescence imaging in the identification of the PGs, mainly in benign disease. Directions of further research should be aimed at improving the technique. Also, more in-depth study of the correlation between the signal and the postoperative function of the PGs will further substantiate the relevance of the technique. In conclusion, NIRF imaging with the use of ICG for intra-operative identification of the PGs and the assessment of its vascularization is feasible and safe and can provide more certainty about the location of the PGs. 


\section{REFERENCES}

1. Bhattacharyya N, Fried MP. Assessment of the morbidity and complications of total thyroidectomy. Arch Otolaryngol Head Neck Surg. 2002;128(4):389-392.

2. Papaleontiou M, Hughes DT, Guo C, Banerjee M, Haymart MR. Population-based assessment of complications following surgery for thyroid cancer. J Clin Endocrinol Metab. 2017;102(7):25432551.

3. Asari R, Passler C, Kaczirek K, Scheuba C, Niederle B. Hypoparathyroidism after total thyroidectomy: a prospective study. Arch Surg. 2008;143(2):132-137. discussion 8.

4. Reeve T, Thompson NW. Complications of thyroid surgery: how to avoid them, how to manage them, and observations on their possible effect on the whole patient. World J Surg. 2000;24(8):971-975.

5. Shah M, Bancos I, Thompson GB, et al. Teriparatide therapy and reduced postoperative hospitalization for postsurgical Hypoparathyroidism. JAMA Otolaryngol Head Neck Surg. 2015;141(9):822-827.

6. Dudley NE. Methylene blue for rapid identification of the parathyroids. $\mathrm{Br}$ Med J. 1971;3(5776):680-681.

7. Patel HP, Chadwick DR, Harrison BJ, Balasubramanian SP. Systematic review of intravenous methylene blue in parathyroid surgery. Br J Surg.2012;99(10):1345-1351.

8. Maguire CA, Sharma A, Alarcon L, et al. Histological features of methylene blue-induced phototoxicity administered in the context of parathyroid surgery. Am J Dermatopathol. 2017;39(8):e110-e115.

9. Kartha SS, Chacko CE, Bumpous JM, Fleming M, Lentsch EJ, Flynn MB. Toxic metabolic encephalopathy after parathyroidectomy with methylene blue localization. Otolaryngol Head Neck Surg. 2006;135(5):765-768.

10. Tummers $Q$, Schepers $A$, Hamming JF, et al. Intraoperative guidance in parathyroid surgery using near-infrared fluorescence imaging and low-dose methylene blue. Surgery. 2015;158(5):13231330.

11. van der Vorst JR, Schaafsma BE, Verbeek FP, et al. Intraoperative near-infrared fluorescence imaging of parathyroid adenomas with use of low-dose methylene blue. Head Neck. 2014;36(6):853-858.

12. Takeuchi S, Shimizu K, Shimizu K Jr, Akasu H, Okamura R. Identification of pathological and normal parathyroid tissue by fluorescent labeling with 5 -aminolevulinic acid during endocrine neck surgery. J Nippon Med Sch.2014;81(2):84-93.

13. Prosst RL, Weiss J, Hupp L, Willeke F, Post S. Fluorescence-guided minimally invasive parathyroidectomy: clinical experience with a novel intraoperative detection technique for parathyroid glands. World J Surg. 2010;34(9):2217-2222.

14. Skrovina M, Bencurik V, Holaskova $E$, et al. Fluorescence angiography in the detection of anastomotic perfusion during rectal and sigmoid resection -preliminary report. Rozhl Chir. 2016;95(10):354-358.

15. Macedo ALV, Schraibman V. Intraoperative near-infrared fluorescent imaging during robotic operations. Einstein. 2016;14(4):577-579.

16. Holloway RW, Abu-Rustum NR, Backes FJ, et al. Sentinel lymph node mapping and staging in endometrial cancer: a Society of Gynecologic Oncology literature review with consensus recommendations. Gynecol Oncol. 2017;146(2):405-415.

17. Suh YJ, Choi JY, Chai YJ, et al. Indocyanine green as a near-infrared fluorescent agent for identifying parathyroid glands during thyroid surgery in dogs. Surg Endosc. 2015;29(9):28112817.

18. Lang BH, Wong $\mathrm{CK}$, Hung HT, Wong KP, Mak KL, Au KB. Indocyanine green fluorescence angiography for quantitative evaluation of in situ para-thyroid gland perfusion and function after total thyroidectomy. Surgery.2017;161(1):87-95. 
19. Vidal Fortuny J, Karenovics W, Triponez F, Sadowski SM. Intra-operative indocyanine green angiography of the parathyroid gland. World J Surg.2016;40(10):2378-2381.

20. Zaidi N, Bucak E, Yazici P, et al. The feasibility of indocyanine green fluorescence imaging for identifying and assessing the perfusion of parathyroid glands during total thyroidectomy. J Surg Oncol. 2016;113(7):775-778.

21. General Assembly of the World Medical A. World medical association declaration of Helsinki: ethical principles for medical research involving human subjects. J Am Coll Dent.2014;81(3): 14-18.

22. Schols RM, Bouvy ND, Masclee AA, van Dam RM, Dejong CH, Stassen LP. Fluorescence cholangiography during laparoscopic cholecystectomy: a feasibility study on early biliary tract delineation. Surg Endosc.2013;27(5):1530-1536.

23. Sound S, Okoh A, Yigitbas H, Yazici P, Berber E. Utility of Indocyanine green fluorescence imaging for intraoperative localization in re-operative parathyroid surgery. Surg Innov. 2015.

24. Yu HW, Chung JW, Yi JW, et al. Intraoperative localization of the parathyroid glands with indocyanine green and firefly ${ }^{\circledast}$ technology during BABA robotic thyroidectomy. Surg Endosc. 2017;31(7):3020-3027.

25. Vidal Fortuny J, Belfontali V, Sadowski SM, Karenovics W, Guigard S, Triponez F. Parathyroid gland angiography with indocyanine green fluorescence to predict parathyroid function after thyroid surgery. Br J Surg. 2016;103(5):537-543.

26. Cui L, Gao Y, Yu H, et al. Intraoperative parathyroid localization with near-infrared fluorescence imaging using Indocyanine green during total para-thyroidectomy for secondary hyperparathyroidism. Sci Rep. 2017;7(1):8193. 
PART IV 
SUMMARY, GENERAL DISCUSSION AND FUTURE PERSPECTIVES 
CHAPTER 12
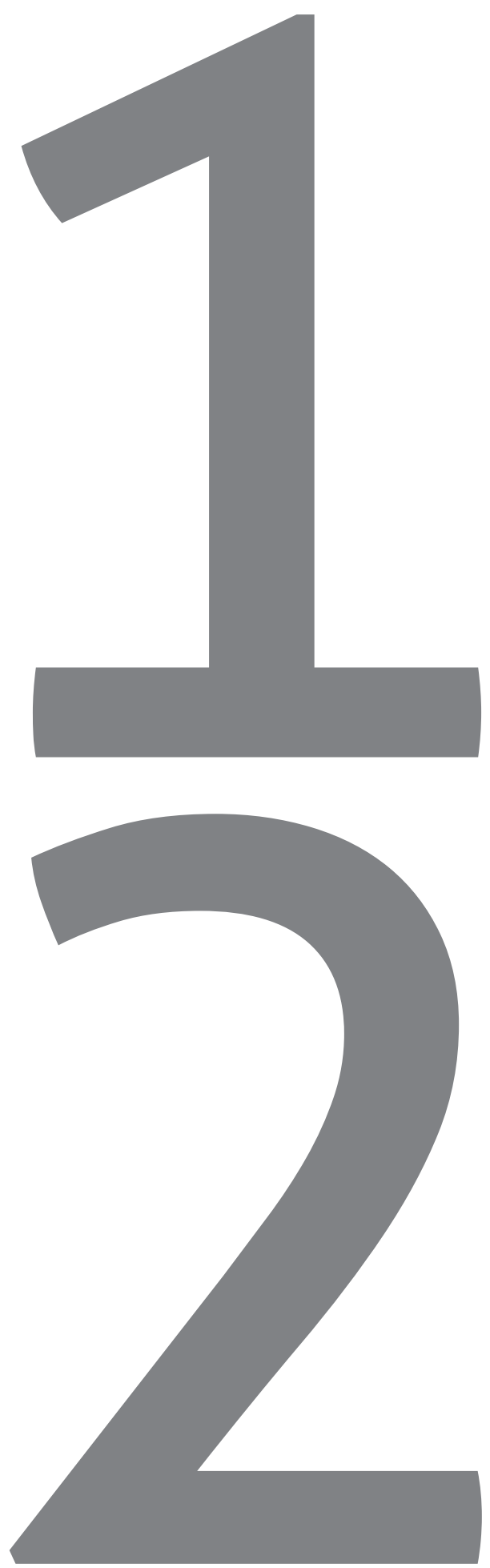
Summary and general discussion 


\section{Summary}

Misidentification of vital anatomical structures such as bile ducts, arteries and ureters may lead to iatrogenic injury. Therefore, detection of these structures is of utmost importance. An optical tool to enhance identification of structures might be helpful in preventing iatrogenic damage. One such optical tool is Near Infra-Red Fluorescence (NIRF) imaging. Preliminary studies, concerning experimental and limited human series, report encouraging results. However, at the start of the studies of this thesis, the technique was yet not standardly incorporated in daily practice. The overall aim of this thesis was to investigate and critically assess some of the possible applications of near infrared fluorescence imaging during surgery in order to prevent iatrogenic damage due to misrecognition of the anatomy.

The first part of the thesis concerns the use of NIRF cholangiography in laparoscopic cholecystectomy. In the second part, we investigated the possibilities of NIRF anatomical imaging in colorectal surgery. In the third part a pilot study is presented exploring the use of NIRF imaging to identify the parathyroid glands and to assess its remaining perfusion at the end of surgery.

\section{PART I NEAR INFRARED FLUORESCENCE CHOLANGIOGRAPHY}

Laparoscopic cholecystectomy is the most commonly performed laparoscopic procedure. The most feared complication in this type of surgery is bile duct injury. Although fortunately not occurring commonly (0.3-0.7\% of cholecystectomies), bile duct injury results in major morbidity and in some cases even mortality. ${ }^{1-9}$ Misidentification of the extrahepatic bile duct anatomy is the main cause for the occurrence of this complication. ${ }^{10}$ The Critical View of Safety (CVS) technique is developed to minimize the chance for misidentification of the anatomy. ${ }^{11}$ Pilot studies have shown that by the use of near infrared fluorescence (NIRF) cholangiography the visualization of the biliary anatomy can be enhanced, and the CVS can be achieved earlier. We assume that the CVS is thus easier to achieve using NIRF. In Chapter 2, the study protocol for an international multicenter randomized controlled trial is presented. This trial should prove whether the use of NIRF cholangiography does help the surgeon by obtaining earlier CVS or not.

When using NIRF cholangiography, great differences in obtained fluorescent signals in the images are found. These differences may originate form (differences in) background fluorescence from the liver and in the penetration depth of the dyes used, but more factors are of influence. ${ }^{12}$ In Chapter 3, currently used techniques during NIRF cholangiography are compared. Factors of influence on the fluorescence intensity are explored in ex vivo experiments combined with knowledge presented in current literature. The factors found 
to be of influence are given dose, timing of ICG administration, route of administration, distance between the laparoscope and the area of interest, patient factors such as BMI and presence of cholecystitis, used system and used software to analyze the data. In this chapter, advise is given on how to use NIRF cholangiography. The concentration of ICG in the bile ducts should be between 0.00195 and $0.025 \mathrm{mg} / \mathrm{ml}$, ICG should be given as early as logistically possible (up to $24 \mathrm{~h}$ before surgery), the laparoscope should be held close to the tissue and the best available system should be used.

In Chapter 4, two new pre-clinical dyes are compared to a third, earlier tested preclinical dye. This third dye, IRDye ${ }^{\circledR} 800 \mathrm{CW}$ was very promising in previous experiments and possible even better than ICG. ${ }^{13}$ However, this dye is expensive, and more expensive compared to the newly developed dyes IRDye ${ }^{\circledR} 800 \mathrm{BK}$ and IRDye ${ }^{\circledR} 800$ NOS. When comparing these three dyes in a pig model, the cystic artery was immediately visible using all three dyes. The cystic duct appeared later, but with the new dyes at least as good as with the use of IRDye ${ }^{\circledR} 800 \mathrm{CW}$. Thereby, IRDye ${ }^{\circledast} 800$ BK and IRDye ${ }^{\circledast} 800$ NOS seem to be good alternatives for IRDye ${ }^{\oplus} 800 \mathrm{CW}$. Our conclusions are based on the subjective evaluation, but also based on the measured target to background ratio.

In Chapter 5, we compared the used methods in literature for objectification of the fluorescent signal. We found that the most commonly used software programs (Osirix and ImageJ) are compatible and comparison of results obtained with these programs seems legitimate. A third used software program (Photoshop) gives less comparable results and these are therefore not easily comparable with the results obtained with the other programs. When using the software programs both grey and blue-value (or green-value depending on the used system) can be measured to determine fluorescence intensity. However, in our images no clear difference between using the grey- and blue- value could be seen. Another aspect is that different formulas are used in literature to assess fluorescence intensity compared to the background. No clear advice can be given on the best formula to be used. Therefore, reporting of both target and background fluorescence intensity in manuscripts is recommended and not only the Target-to-background ratio. This will enable researchers and readers to make a more proper comparison of results between studies.

\section{PART II NEAR INFRARED FLUORESCENCE IMAGING IN COLORECTAL SURGERY}

During colorectal surgery there are two complications surgeons fear most. These two complications are anastomotic leakage in the patients in whom an anastomosis is made and ureter damage in pelvic surgery. Unfortunately, reported incidence of anastomotic 
leakage is high, namely $3-15 \%$ in colorectal surgery. ${ }^{14-21}$ Anastomotic leakage is a very serious complication since it has a great impact on the patient by increasing morbidity, often requiring a second surgery, but also mortality rates are high. ${ }^{16,20-22}$ Several risk factors for anastomotic leakage have been identified, of which an important factor is perfusion of the bowel segments adjacent to the anastomosis. ${ }^{23-25} \mathrm{~A}$ good perfusion is required for adequate anastomotic healing. Since the perfusion can be visualized using near infrared fluorescence imaging, it is thought that the use of near infrared fluorescence imaging can prevent part of the anastomotic leakages. In Chapter 6, we investigated this hypothesis in a systematic review and found that the use of NIRF angiography in anastomotic colorectal surgery seems easily applicable. After NIRF angiography, the resection margin was changed in $10.8 \%$ of cases and a significant lower anastomotic leak rate was found in published literature when using NIRF angiography. Especially an initially bright fluorescent signal seems to be predictive for good anastomotic healing.

In our systematic review in chapter 6, we found that in most studies, the place of resection margin after NIRF angiography was based on the subjective view of the surgeon: whether the bowel was 'fluorescent enough'. However, a clear cut-off value of fluorescence intensity indicating sufficient perfusion is not available yet by would be desirable to base such decisions on rather than on a subjective view.

In Chapter $\mathbf{7}$ therefore, we explored whether the fluorescent signal correlates to serum markers of anastomotic leakage and postoperative inflammation such as CRP, I-FABP and calprotectin. In the heterogenous pilot-group of 30 colorectal cancer-patients we included, no relation between the fluorescence intensity and the inflammatory markers studied could be found. However, a higher measured fluorescence intensity of the proximal bowel compared to the background both before and after surgery was found in the patients who did not develop anastomotic leakage. Also, a subjective scoring of the surgeon during surgery just before transection of the proximal bowel was higher in the patients who did not develop anastomotic leakage.

A second major complication after colorectal surgery is iatrogenic ureter damage. In this surgery, the ureter can be very close to the surgical field and may be hard to identify. Identification of the ureter is the most important step in avoiding ureter damage. By using a near infrared dye that is cleared by the kidneys, the ureters may be visualized during surgery and thereby lessen the chance of iatrogenic ureter damage. In Chapter 8, we investigated the feasibility of NIRF imaging with methylene blue to visualize the ureter in patients undergoing elective laparoscopic colorectal surgery. This dye is registered for clinical use and cheap: NIRF using this dye would be a very practical and promising technique. The only drawback was that a dedicated filter had to be developed, as the commercially available 
equipment didn't have filters for the appropriate wavelength. Ten patients were included, and different doses were tested. We could detect the ureter in five patients, who received the highest doses, namely between 0.75 and $1 \mathrm{mg} / \mathrm{kg}$. However, in all five cases the ureter was also visible in white light before detection in NIRF light. The results were disappointing. Therefore, the use of NIRF imaging with methylene blue in these cases was not considered of added value in clinical practice.

A potent dye with excitation and emission in the range of ICG would be preferable as the commercially available equipment can be used for obtaining the fluorescent signal. In Chapters 9 and 10, we explored the use of two new such dyes. These are still pre-clinical dyes, emitting fluorescent light at around $800 \mathrm{~nm}$ instead of the $688 \mathrm{~nm}$ at which methylene blue emits fluorescent light. The wavelength of $800 \mathrm{~nm}$ is more in the near infrared range and thus has a higher penetration depth. Based on this theory we expected to see the ureters more clearly using these new dyes, IRDye ${ }^{\circledR} 800 \mathrm{BK}$ and IRDye ${ }^{\circledR} 800 \mathrm{NOS}$. We compared these with another, earlier tested preclinical dye, IRdye ${ }^{\circledast} 800 \mathrm{CW}$ in a pig model. In these experiments three pigs were used, one of the three dyes each. These three dyes are cleared by the kidneys and showed a bright fluorescent signal, very clearly delineating the ureters in the pigs, especially during pulsatile movement of urine through the ureter. IRDye ${ }^{\circledast} 800 \mathrm{BK}$ seemed to give the best results of these two new dyes and is further explored in Chapter 10. Three different doses of the IRDye800BK were administered in three different pigs to search for the optimal dose. The used doses were $3 \mathrm{mg}, 6 \mathrm{mg}$ and $12 \mathrm{mg}$. The results obtained with these three doses were comparable. Also, the dye was used in explanted human and pig ureters to see if the penetration depth of the emitted light from the dye was high enough to penetrate through the human ureter. We found that this was possible, but that the presence of fat on the ureter is a limiting factor.

\section{PART III NEAR INFRARED FLUORESCENCE IMAGING IN THYROID SURGERY}

In thyroid surgery, iatrogenic injury of the parathyroid glands should be prevented. Here again, the key to prevention is identification of the anatomical structure, the parathyroid gland. However, in this type of surgery, only identifying is not enough to prevent injury. In some cases, the parathyroid glands are identified and kept in the patient, but due to manipulation of the thyroid, the blood supply towards the parathyroid is impaired, resulting in an insufficient remaining function of the parathyroid glands. In Chapter $\mathbf{1 1}$ we evaluated the possibility of both identifying the parathyroid glands and assessing post-thyroidectomy perfusion of the parathyroid gland. In 30 (hemi) thyroidectomies, NIRF imaging was used. This technique was found safe and easily applicable. In 17 surgeries NIRF imaging was of added value, mainly due to providing more certainty to the operating surgeon about the 
location and remaining function of the parathyroid glands. In all patients, normal calcium levels were found 6 hours after surgery, however a temporary drop in calcium level the day after surgery occurred in three patients in whom the average TBR was lower compared to the measured TBR in the remaining patients. The calcium levels in these patients restored after two weeks. 


\section{General discussion and future perspectives}

\section{PART I NEAR INFRARED FLUORESCENCE CHOLANGIOGRAPHY}

The RCT as described in Chapter $\mathbf{2}$ is meant to identify the advantages of NIRF in laparoscopic cholecystectomy. The main aim of applying this imaging technique is to make the operation safer in terms of reduction of bile duct injury (BDI). However, due to its low incidence BDI can unfortunately not serve as a primary endpoint for the study described in Chapter $\mathbf{2}$. The decision to choose a surrogate primary endpoint such as time till CVS in our RCT can be considered as a limitation of this study.

Depending of the future outcomes of this trial, the use of NIRF cholangiography should be incorporated in clinical practice or not, as is suggested already in current literature that appeared after publishing this study protocol.12,26-28 Reduced operating time but also enhanced surgeon's satisfaction will promote the use of NIRF for this indication. This opens the possibility of future large prospective registration studies that will reveal whether indeed with this technique surgery has also become safer.

In Chapter $\mathbf{3}$ some clear advice is given on how to perform NIRF imaging and how to obtain the best images with current techniques. However, this advice is not perfect. For example, holding the laparoscope close to the cystic duct gives a brighter fluorescent signal of the cystic duct. But also, the fluorescent signal from the liver in the background will increase by holding the laparoscope closer. The background fluorescence-noise can be reduced when administrating the dye earlier, preferably the day before surgery. ${ }^{29}$ Unfortunately, this is not always logistically feasible. A solution to this problem of background fluorescence might come from using another way of administration, as is shortly discussed in the article. By injecting the fluorescent dye directly into the gallbladder, the gallbladder and cystic duct become fluorescent without the liver having to clear the fluorescent dye from the blood and thereby becoming fluorescent. This is investigated by Liu et $\mathrm{al}^{30}$ and seems to be a promising method which merits further evaluation. In this way, one does not have to decide beforehand whether or not to use NIRF cholangiography during a laparoscopic cholecystectomy, but this technique can be started in selected, difficult, cases when needed. On the other hand, from our personal experience observing the surgeon's satisfaction resulting from the clearer anatomical picture and ease of use of the technique, we expect most surgeons will prefer applying the technique in all cases instead of selected ones. This of course, will be influenced by the results of trials, such as the FALCON trial described in chapter 2.

Apart from the used route of administration, also other fluorescent dyes need to be explored further. In current practice, ICG is used for nearly all applications of identifying normal anatomy. There is a lot of experience with this dye and this dye is generally assessed 
as a very safe dye, although in rare instances, Indocyanine green can cause an allergic reaction due to the iodine present in the dye..$^{31,32}$

Chapter 4 explores dyes that may provide a better signal in NIRF cholangiography compared to current practice. One of the downsides of this study is that we had to use an animal model to investigate these dyes and were not able to perform human testing yet, as the dyes are not registered for use in humans yet. Fortunately, our results are part of the process of obtaining FDA approval. As soon as the dyes IRDye ${ }^{\circledast} 800$ BK and IRDye ${ }^{\circledast}$ 800 NOS become available for clinical practice their role in fluorescence cholangiography in humans should be determined. IRDye ${ }^{\circledR} 800 \mathrm{CW}$, the predecessor of the new dyes, is also promising for bile duct imaging, but a major downside is its high cost. An interesting property of IRDye ${ }^{\circledast} 800 \mathrm{CW}$ however is that it can be coupled to another substance. It can for example be coupled to bevacizumab in order to enable targeted imaging, e.g. to visualize colorectal neoplasms. ${ }^{33}$ Another application is the conjugation of cetuximab-IRDye ${ }^{\circledR} 800 \mathrm{CW}$ to identify metastatic disease in lymph nodes in patients with head and neck cancer. ${ }^{34}$ These very promising and interesting applications are beyond the scope of this thesis but will be subject of future research.

The experiment described in Chapter $\mathbf{5}$ focusses on the comparability of the fluorescence signal as reported between studies performed by different study groups. Used techniques in assessment of fluorescence intensity are explored. In this experiment also, the use of greyvalues and blue-values are compared. These results did not differ as much as the authors expected. Caution must however be taken that apart from the used software and chosen formula the selection of regions of Interest is also of influence on the measured fluorescence intensity.

In summary, the future concerning NIRF-cholangiography is dependent on the outcomes of randomized controlled trials such as presented in Chapter $\mathbf{2}$ but should also include a search for ways to improve currently used techniques further, for example by using better fluorescent dyes, optimizing equipment and keeping in mind the factors of influence on the signal during application of the technique.

\section{PART II NEAR INFRARED FLUORESCENCE IMAGING IN COLORECTAL SURGERY}

Regarding NIRF for colorectal perfusion measurement, large clinical trials are needed. Since the publication of the systematic review presented in Chapter 6, a large cohort study is published. ${ }^{35}$ This multicenter study included a heterogenous group of 504 patients 
undergoing colorectal surgery involving resection and anastomosis. In all included patients, NIRF was used to assess perfusion just before and after construction of the anastomosis. An anastomotic leak rate of $2.6 \%$ was found in this cohort. Compared to reported incidence in literature, this is very low. It is very well possible that in persisting anastomotic leakage cases other risk factors than impaired perfusion play a role, since anastomotic leakage is a multifactorial problem.

A randomized controlled trial concerning the technique is not published yet but a research group in Leeds took the initiative to start such a randomized trial. ${ }^{36}$ The results of this trial are planned to be presented in 2021 and will give us further arguments whether or not to use this technique in all anastomotic colorectal surgery. Awaiting the results of such trials, one could defend the standard use of NIRF during colorectal anastomotic surgery, given the promising results of clinical studies so far. Another way to go, apart from an RTC, is a prospective registry in which all such patients are collected. This will give insight in the results of the technique in daily practice.

The information of previous studies that a higher fluorescence intensity at the bowel ends adjacent to the (future) anastomosis at certain time points was related to less anastomotic leakage, brought us to the study as performed in Chapter 7. The limitation of all studies so far is that no objective clear cut-off point for adequate perfusion has been studied or identified. We hoped to find a correlation between the objective value of the fluorescent signal and other parameters indicative of inflammation and predictive of anastomotic leakage. Unfortunately, no such correlation was found. This is in part due to the small pilot. Another factor may be that the other parameters studied are not as predictive as previous studies suggested. A next step is to investigate in a larger cohort of NIRF patients what an optimal cut-off value would be to reassure the surgeon that the bowel is fluorescent 'enough'. And even further: to come to an absolute cut-off value that can be actually used during surgery. During our experiments, such a measurement for use during surgery was not available, as our measurements were done after surgery. However, the technique for intraoperative evaluation of the fluorescence intensity is being developed and tested. Diana et al explored the possibility of real-time fluorescence measurement in a pig model. ${ }^{37,38}$ Not only the eventual signal itself, but also the time it takes for the tissue to produce this signal seems indicative of adequate perfusion. To date, no results on in-human use of this technique are reported yet.

For now, it seems that we can and have to depend on the subjective assessment of fluorescence intensity by the surgeon, just before transection of the proximal bowel. As soon as more data on in-human intra-operative objective fluorescence intensity measurement is available, a cut-off value should be determined for more reliable use of the technique. 
From Chapter $\mathbf{8}$ on, we evaluated the possibility of visualizing the ureter during laparoscopic colorectal surgery. The experiments we performed on dyes for ureteral NIRF imaging are promising and relevant as this technique might help to prevent ureteral injury and diminish this feared complication. The first logical step was to test the performance of methylene blue, as this dye is already registered for human use, albeit in other indications. A downside of the use of methylene blue is its excitation and emission wavelength. Although called a near infrared dye, the wavelengths concerning this dye are 668 and $688 \mathrm{~nm}$ for excitation and emission respectively, which is on the lower limit of being in the near infrared range. ${ }^{39}$ This requires equipment that is specifically adjusted with adequate filters. For other applications, a dye with a wavelength of $800 \mathrm{~nm}$ which is more clearly in the near infrared range is chosen because of a lack of disturbance of the normal white light image, and a higher penetration depth. ${ }^{39}$ Such dye is indocyanine green, which is cleared solely by the liver, thus this dye will not end up in the ureters after intravenous administration. ICG can be brought in the ureters by the use of ureteral catheter and ureters can be visualized using this method. ${ }^{40,41}$ However, ureter catheter placement is an invasive procedure, requires additional expertise and adds to operation time. ${ }^{42}$ While using a dye cleared by the kidneys is easy to apply without added surgical time. Thus, a dye emitting light at around $800 \mathrm{~nm}$ which is cleared by the kidneys is desired. Three of these dyes are tested in Chapters $\mathbf{9}$ and 10. This concerned experimental dyes tested in a pig model. The experimental dyes tested are promising, because of their characteristics concerning their wavelengths, their performance with regard to the very clearly observed signal and their price. The images obtained were subjectively by far superior to the images obtained with methylene blue.

Future research should be performed in humans as soon as approval of authorities is obtained to use these dyes in humans.

Apart from the mentioned dyes, other possible new dyes are also explored. Mahalingam et al published in 2018 an article in which they describe the use of 'Ureter-glow' to visualize the ureters. ${ }^{43}$ This new dye is highly water soluble and has an excitation wavelength of $800 \mathrm{~nm}$ and emission wavelength of $830 \mathrm{~nm}$. The dye was tested in mice and pigs and seems promising. ${ }^{43}$ Whether the signal will be of the same quality in the human situation, with the presence of peri-ureteral fat, will have to be awaited as is the case with the dyes tested in our own experiments.

In summary, currently we do not have an ideal tool to visualize the ureters in human colorectal surgery using near infrared fluorescence imaging. Nevertheless, promising experimental dyes have been tested with good results. Future research is needed to test the new fluorescent dyes for ureter imaging as soon as authorities allow human application. 


\section{PART III NEAR INFRARED FLUORESCENCE IMAGING IN THYROID SURGERY}

Concerning the application of NIRF in thyroid surgery we concluded in Chapter $\mathbf{1 1}$ that in 17 of 30 surgeries the technique was of added value, increasing the certainty of the location of the parathyroid glands and their function.

One can debate whether such reassuring the surgeon is enough for daily clinical use. To our opinion, this technique should be further improved before entering daily practice. Currently, the background signal of the thyroid is quite high. Another downside of the technique was that in our patients, this background fluorescence was especially high in patients with lymph node metastases. Especially in these cases parathyroid glands may be hard to distinguish from surrounding tissue by the naked eye and thus a technique to enhance visualization might be most helpful. Recently, other positive research results were published. A randomized controlled trial was performed by Fortuny et al. ${ }^{44}$ This research group used a lower dose $(2.5 \mathrm{mg})$ compared to our experiment $(7.5 \mathrm{mg})$ and a different fluorescence imaging system (Pinpoint ${ }^{\circledR}$ instead of the Karl Storz system). Adult patients were included undergoing total thyroidectomy or completion thyroidectomy in whom an ICG score of 2 was observed in at least one identified parathyroid gland, meaning the parathyroid gland was bright fluorescent. These patients were randomized in two groups. The control group recieved standard follow-up with calcium and PTH measurements on day 1 and 10-15 postoperatively and oral supplementation with $1 \mathrm{mg}$ calcium and 800 units 25-hydroxyvitamin $\mathrm{D}$ twice daily upon the postoperative appointment after 10 to 15 days. In the intervention group patients were assessed for symptoms of hypocalcemia, but no blood test was done the day after surgery, nor supplementation of calcium and vitamin D was given. On day 10-15, calcium and PTH levels were measured to confirm that levels were normal. The results show that in all included patients postoperative calcium levels were normal. The conclusion of this RCT is that the use of NIRF angiography in thyroid surgery obviates the need for postoperative measurement of calcium and PTH and supplementation with calcium in patients with at least one well perfused parathyroid gland. ${ }^{44}$

Other methods for parathyroid visualization are being assessed. One of these methods is using methylene blue instead of ICG as a fluorescent dye. ${ }^{45}$ However, high doses of Methylene blue are needed, causing a risk for serious hemodynamic complications caused by methylene blue and its effectiveness is not proven. Because of this risk and limited benefit, the use of this technique is discouraged. ${ }^{46}$ Using a lower dose would lower the risk of side effects but another disadvantage of the use of methylene blue as a fluorescent dye is that equipment with other filters than used with ICG are needed because of the different excitation and emission wavelength of the dyes. ${ }^{47} \mathrm{~A}$ comparative study between methylene blue and ICG as a fluorescent dye in this application should be performed to determine what dye to prefer. 
Autofluorescence is another promising method for intra-operative parathyroid identification. ${ }^{48-50}$ Using this technique, no exogenous fluorescent dye administration is needed. However, for perfusion assessment of the remaining parathyroid gland an intravenously administered fluorescent dye is still needed and might then as well be used for identification of the parathyroid glands, instead of using multiple systems and techniques. A comparative study by Kahramangil et al ${ }^{51}$ concluded that earlier detection with autofluorescence imaging was possible, but autofluorescence and ICG NIRF imaging have similar high detection rates.

In conclusion, in this thesis we critically assessed the possible applications of near infrared imaging during laparoscopic cholecystectomy, colorectal anastomotic surgery and thyroid surgery. We described positive results of this technique, points of attention and future directions of development and research. Randomized trials but also prospective cohorts will give valuable information. The availability of potent dyes for human use will further enhance its application. It seems likely that for the next years this technique will not only remain but will be increasingly incorporated in daily practice. 


\section{REFERENCES}

1. Bobkiewicz A, Krokowicz L, Banasiewicz T, Koscinski T, Borejsza-Wysocki M, Ledwosinski W, et al. latrogenic bile duct injury. A significant surgical problem. Assessment of treatment outcomes in the department's own material. Pol Przegl Chir. 2014;86(12):576-83.

2. Boerma D, Rauws EA, Keulemans YC, Bergman JJ, Obertop H, Huibregtse K, et al. Impaired quality of life 5 years after bile duct injury during laparoscopic cholecystectomy: a prospective analysis. Ann Surg. 2001;234(6):750-7.

3. Booij KA, de Reuver PR, Yap K, van Dieren S, van Delden OM, Rauws EA, et al. Morbidity and mortality after minor bile duct injury following laparoscopic cholecystectomy. Endoscopy. 2015;47(1):40-6.

4. Dolan JP, Diggs BS, Sheppard BC, Hunter JG. Ten-year trend in the national volume of bile duct injuries requiring operative repair. Surg Endosc. 2005;19(7):967-73.

5. Fletcher DR, Hobbs MS, Tan P, Valinsky LJ, Hockey RL, Pikora TJ, et al. Complications of cholecystectomy: risks of the laparoscopic approach and protective effects of operative cholangiography: a population-based study. Ann Surg. 1999;229(4):449-57.

6. Flum DR, Dellinger EP, Cheadle A, Chan L, Koepsell T. Intraoperative cholangiography and risk of common bile duct injury during cholecystectomy. JAMA. 2003;289(13):1639-44.

7. Landman MP, Feurer ID, Moore DE, Zaydfudim V, Pinson CW. The long-term effect of bile duct injuries on health-related quality of life: a meta-analysis. HPB (Oxford). 2013;15(4):252-9.

8. Nuzzo G, Giuliante F, Giovannini I, Ardito F, D'Acapito F, Vellone M, et al. Bile duct injury during laparoscopic cholecystectomy: results of an Italian national survey on 56591 cholecystectomies. Archives of surgery (Chicago, III : 1960). 2005;140(10):986-92.

9. Waage A, Nilsson M. latrogenic bile duct injury: a population-based study of 152776 cholecystectomies in the Swedish Inpatient Registry. Archives of surgery (Chicago, III : 1960). 2006;141(12):1207-13.

10. Way LW, Stewart L, Gantert W, Liu K, Lee CM, Whang K, et al. Causes and prevention of laparoscopic bile duct injuries: analysis of 252 cases from a human factors and cognitive psychology perspective. Annals of surgery. 2003;237(4):460-9.

11. Strasberg SM, Hertl M, Soper NJ. An analysis of the problem of biliary injury during laparoscopic cholecystectomy. J Am Coll Surg. 1995;180(1):101-25.

12. Pesce A, Diana M. Critical View of Safety During Laparoscopic Cholecystectomy: From the Surgeon's Eye to Fluorescent Vision. Surg Innov. 2018;25(3):197-8.

13. Schols RM, Lodewick TM, Bouvy ND, van Dam DA, Meijerink WJ, van Dam GM, et al. Nearinfrared fluorescence laparoscopy of the cystic duct and artery in pigs: performance of a preclinical dye. J Laparoendosc Adv Surg Tech A. 2014;24(5):318-22.

14. Leahy J, Schoetz D, Marcello P, Read T, Hall J, Roberts P, et al. What is the risk of clinical anastomotic leak in the diverted colorectal anastomosis? Journal of gastrointestinal surgery: official journal of the Society for Surgery of the Alimentary Tract. 2014;18(10):1812-6.

15. Leichtle SW, Mouawad NJ, Welch KB, Lampman RM, Cleary RK. Risk factors for anastomotic leakage after colectomy. Diseases of the colon and rectum. 2012;55(5):569-75.

16. Midura EF, Hanseman D, Davis BR, Atkinson SJ, Abbott DE, Shah SA, et al. Risk factors and consequences of anastomotic leak after colectomy: a national analysis. Diseases of the colon and rectum. 2015;58(3):333-8.

17. Morse BC, Simpson JP, Jones YR, Johnson BL, Knott BM, Kotrady JA. Determination of independent predictive factors for anastomotic leak: analysis of 682 intestinal anastomoses. American journal of surgery. 2013;206(6):950-5; discussion 5-6.

18. Ruggiero R, Sparavigna L, Docimo G, Gubitosi A, Agresti M, Procaccini E, et al. Post-operative peritonitis due to anastomotic dehiscence after colonic resection. Multicentric experience, retrospective analysis of risk factors and review of the literature. Annali italiani di chirurgia. 2011;82(5):369-75. 
19. Sultan R, Chawla T, Zaidi M. Factors affecting anastomotic leak after colorectal anastomosis in patients without protective stoma in tertiary care hospital. JPMA The Journal of the Pakistan Medical Association. 2014;64(2):166-70.

20. Turrentine FE, Denlinger CE, Simpson VB, Garwood RA, Guerlain S, Agrawal A, et al. Morbidity, mortality, cost, and survival estimates of gastrointestinal anastomotic leaks. Journal of the American College of Surgeons. 2015;220(2):195-206.

21. Buchs NC, Gervaz P, Secic M, Bucher P, Mugnier-Konrad B, Morel P. Incidence, consequences, and risk factors for anastomotic dehiscence after colorectal surgery: a prospective monocentric study. International journal of colorectal disease. 2008;23(3):265-70.

22. Nachiappan S, Askari A, Currie A, Kennedy RH, Faiz O. Intraoperative assessment of colorectal anastomotic integrity: a systematic review. Surgical endoscopy. 2014;28(9):2513-30.

23. Karliczek A, Benaron DA, Zeebregts CJ, Wiggers T, van Dam GM. Intraoperative ischemia of the distal end of colon anastomoses as detected with visible light spectroscopy causes reduction of anastomotic strength. The Journal of surgical research. 2009;152(2):288-95.

24. Pommergaard HC, Achiam MP, Burcharth J, Rosenberg J. Impaired blood supply in the colonic anastomosis in mice compromises healing. International surgery. 2015;100(1):70-6.

25. Wu JH, Rong ZX, Zhu DJ, Chen XW, Ren BJ. [Laparoscopic anterior resection of rectal carcinoma with preservation of the left colonic artery]. Nan fang yi ke da xue xue bao = Journal of Southern Medical University. 2009;29(6):1249-50.

26. Renz BW, Bosch F, Angele MK. Bile Duct Injury after Cholecystectomy: Surgical Therapy. Visc Med. 2017;33(3):184-90.

27. Conrad C, Wakabayashi G, Asbun HJ, Dallemagne B, Demartines N, Diana M, et al. IRCAD recommendation on safe laparoscopic cholecystectomy. J Hepatobiliary Pancreat Sci. 2017;24(11):603-15.

28. Pesce A, Latteri S, Barchitta M, Portale TR, Di Stefano B, Agodi A, et al. Near-infrared fluorescent cholangiography - real-time visualization of the biliary tree during elective laparoscopic cholecystectomy. HPB (Oxford). 2018;20(6):538-45.

29. Verbeek FP, Schaafsma BE, Tummers QR, van der Vorst JR, van der Made WJ, Baeten $\mathrm{Cl}$, et al. Optimization of near-infrared fluorescence cholangiography for open and laparoscopic surgery. Surg Endosc. 2014;28(4):1076-82.

30. LiuYY,LiaoCH,DianaM,WangSY,KongSH,YehCN, etal.Near-infraredcholecystocholangiography with direct intragallbladder indocyanine green injection: preliminary clinical results. Surg Endosc. 2018;32(3):1506-14.

31. Hope-Ross M, Yannuzzi LA, Gragoudas ES, Guyer DR, Slakter JS, Sorenson JA, et al. Adverse reactions due to indocyanine green. Ophthalmology. 1994;101(3):529-33.

32. Speich R, Saesseli B, Hoffmann U, Neftel KA, Reichen J. Anaphylactoid reactions after indocyanine-green administration. Ann Intern Med. 1988;109(4):345-6.

33. Hartmans E, Tjalma JJJ, Linssen MD, Allende PBG, Koller M, Jorritsma-Smit A, et al. Potential Red-Flag Identification of Colorectal Adenomas with Wide-Field Fluorescence Molecular Endoscopy. Theranostics. 2018;8(6):1458-67.

34. Rosenthal EL, Moore LS, Tipirneni K, de Boer E, Stevens TM, Hartman YE, et al. Sensitivity and Specificity of Cetuximab-IRDye800CW to Identify Regional Metastatic Disease in Head and Neck Cancer. Clin Cancer Res. 2017;23(16):4744-52.

35. Ris F, Liot E, Buchs NC, Kraus R, Ismael G, Belfontali V, et al. Multicentre phase II trial of nearinfrared imaging in elective colorectal surgery. The British journal of surgery. 2018.

36. IntAct: Intraoperative Fluorescence Angiography to Prevent Anastomotic Leak in Rectal Cancer Surgery. [Available from: https://www.isrctn.com/ISRCTN13334746?q=intact-ifa\&filters=\&sort $=$ \&offset=1\&totalResults=1\&page $=1$ \&pageSize $=10 \&$ searchType=basic-search .

37. Diana M, Noll E, Charles AL, Diemunsch P, Geny B, Liu YY, et al. Precision real-time evaluation of bowel perfusion: accuracy of confocal endomicroscopy assessment of stoma in a controlled hemorrhagic shock model. Surgical endoscopy. 2017;31(2):680-91. 
38. Diana M, Noll E, Diemunsch P, Dallemagne B, Benahmed MA, Agnus V, et al. Enhancedreality video fluorescence: a real-time assessment of intestinal viability. Annals of surgery. 2014;259(4):700-7.

39. Sakudo A. Near-infrared spectroscopy for medical applications: Current status and future perspectives. Clin Chim Acta. 2016;455:181-8.

40. Lee $Z$, Moore B, Giusto L, Eun DD. Use of indocyanine green during robot-assisted ureteral reconstructions. Eur Urol. 2015;67(2):291-8.

41. Park H, Farnam RW. Novel Use of Indocyanine Green for Intraoperative, Real-time Localization of Ureter During Robot-Assisted Excision of Endometriosis. J Minim Invasive Gynecol. 2015;22(6S):S69.

42. Speicher PJ, Goldsmith ZG, Nussbaum DP, Turley RS, Peterson AC, Mantyh CR. Ureteral stenting in laparoscopic colorectal surgery. The Journal of surgical research. 2014;190(1):98-103.

43. Mahalingam SM, Dip F, Castillo M, Roy M, Wexner SD, Rosenthal RJ, et al. Intra-operative ureter visualization using a novel near infrared fluorescent dye. Mol Pharm. 2018.

44. Vidal Fortuny J, Sadowski SM, Belfontali V, Guigard S, Poncet A, Ris F, et al. Randomized clinical trial of intraoperative parathyroid gland angiography with indocyanine green fluorescence predicting parathyroid function after thyroid surgery. The British journal of surgery. 2018;105(4):350-7.

45. Han N, Bumpous JM, Goldstein RE, Fleming MM, Flynn MB. Intra-operative parathyroid identification using methylene blue in parathyroid surgery. Am Surg. 2007;73(8):820-3.

46. Patel HP, Chadwick DR, Harrison BJ, Balasubramanian SP. Systematic review of intravenous methylene blue in parathyroid surgery. The British journal of surgery. 2012;99(10):1345-51.

47. Hillary SL, Guillermet S, Brown NJ, Balasubramanian SP. Use of methylene blue and nearinfrared fluorescence in thyroid and parathyroid surgery. Langenbeck's archives of surgery / Deutsche Gesellschaft fur Chirurgie. 2018;403(1):111-8.

48. McWade MA, Paras C, White LM, Phay JE, Solorzano CC, Broome JT, et al. Label-free intraoperative parathyroid localization with near-infrared autofluorescence imaging. J Clin Endocrinol Metab. 2014;99(12):4574-80.

49. Kahramangil B, Dip F, Benmiloud F, Falco J, de La Fuente M, Verna S, et al. Detection of Parathyroid Autofluorescence Using Near-Infrared Imaging: A Multicenter Analysis of Concordance Between Different Surgeons. Ann Surg Oncol. 2018;25(4):957-62.

50. Kim SW, Lee HS, Ahn YC, Park CW, Jeon SW, Kim CH, et al. Near-Infrared Autofluorescence Image-Guided Parathyroid Gland Mapping in Thyroidectomy. Journal of the American College of Surgeons. 2018;226(2):165-72.

51. Kahramangil B, Berber E. Comparison of indocyanine green fluorescence and parathyroid autofluorescence imaging in the identification of parathyroid glands during thyroidectomy. Gland Surg. 2017;6(6):644-8. 
PART V 
ADDENDUM 


\section{Nederlandse Samenvatting}

Wanneer anatomische structuren zoals galwegen, arteriën en ureteren tijdens operaties niet goed herkend worden kan dit leiden tot iatrogene schade. Het is daarom van groot belang om deze anatomie optimaal te detecteren. Een hulpmiddel dat het identificeren van deze structuren makkelijker maakt zou er daarom voor kunnen zorgen dat de kans op iatrogene schade verminderd wordt. Het gebruik van nabij-infrarode fluorescentie (NIRF) beeldvorming zou zo'n hulpmiddel kunnen zijn. Eerdere studies in de (dier)experimentele setting laten veelbelovende resultaten zien. Deze techniek werd voorafgaand aan het schrijven van deze thesis echter nog niet in de dagelijkse praktijk gebruikt. Het doel van deze thesis is het onderzoeken en kritisch beoordelen van de mogelijke toepassingen van NIRF-beeldvorming in het voorkomen van iatrogene schade als gevolg van misidentificatie van de anatomie.

Het eerst deel van de thesis omvat het gebruik van NIRF-cholangiografie bij laparoscopische cholecystectomie. In het tweede deel worden de mogelijkheden van NIRFbeeldvorming ter voorkoming van iatrogene schade in colorectale chirurgie onderzocht. Het derde deel betreft het gebruik van NIRF-beeldvorming ter identificatie van de bijschildklier en voor de resterende perfusie van de bijschildklier na een schildklieroperatie.

\section{DEEL 1 NABIJ-INFRARODE FLUORESCENTIE CHOLANGIOGRAFIE}

Laparoscopische cholecystectomie is de meest uitgevoerde laparoscopische procedure. Hierbij is schade aan de galwegen de meest gevreesde complicatie. Gelukkig komt deze complicatie niet vaak voor (0.3-07\%), maar deze zorgt wel voor veel morbiditeit en soms zelfs mortaliteit. ${ }^{1-9}$ Het niet juist interpreteren van de anatomie van de extra-hepatische galwegen wordt gezien als de belangrijkste oorzaak voor het optreden van deze complicatie. ${ }^{10} \mathrm{De}$ 'Critical view of safety'(CVS)- techniek is ontwikkeld om galwegletsel te voorkomen, door de kans op misidentificatie te minimaliseren." Pilotstudies hebben laten zien dat het gebruik van NIRF-cholangiografie de anatomie van de galwegen duidelijker maakt, en dat zo de CVS eerder bereikt kan worden. In hoofdstuk 2 wordt het studieprotocol voor een internationale multicenter gerandomiseerde studie gepresenteerd. Het doel van deze studie is om te evalueren of het gebruik van NIRF-cholangiografie leidt tot het eerder bereiken van de CVS.

Bij het gebruik van NIRF-cholangiografie worden grote verschillen in het behaalde fluorescentiesignaal gevonden. Deze verschillen kunnen komen door verschillen in de achtergrondfluorescentie van de lever, de penetratiediepte van de gebruikte kleurstof en verschillende andere factoren. ${ }^{12}$ In hoofdstuk 3 worden de technieken die nu gebruikt 
worden voor NIRF-cholangiografie met elkaar vergeleken. Factoren van invloed op de fluorescentie-intensiteit worden onderzocht in ex vivo experimenten, gecombineerd met de kennis uit de huidige literatuur. De volgende beïnvloedende factoren zijn gevonden: dosis, timing van de indocyanine groen (ICG)-toediening, toedieningsweg, afstand tussen de laparoscoop en de kleurstof, patiëntfactoren (zoals BMI), aanwezigheid van ontsteking, het gebruikte systeem en de software om de beelden te analyseren. In dit hoofdstuk wordt op basis van deze bevindingen een advies gegeven hoe NIRF-cholangiografie toe te passen. Zo moet er gestreefd worden naar een concentratie ICG in de gal tussen de 0.00195 en $0.025 \mathrm{mg} / \mathrm{ml}$, moet de ICG zo vroeg mogelijk toegediend worden, moet de laparoscoop dicht bij het te beoordelen weefsel gehouden worden en dient het best beschikbare systeem gebruikt te worden.

In hoofdstuk 4 worden twee nieuwe, preklinische fluorescente kleurstoffen vergeleken met een derde, eerder geteste preklinische fluorescente kleurstof. Deze derde stof, IRDye ${ }^{\circledR}$ 800CW (LICOR Biotechnology, Lincoln, United States), was zeer veelbelovend in eerdere experimenten en leek zelfs beter dan het ICG dat men in de huidige praktijk gebruikt. ${ }^{13}$ Echter, deze kleurstof is erg duur. De nieuwere kleurstoffen IRDye ${ }^{\oplus} 800$ BK $^{\text {en IRDye }}{ }^{\circledR} 800$ NOS zijn een stuk goedkoper. Deze drie kleurstoffen worden met elkaar vergeleken in een studie met varkens, waarbij de arteria cystica direct zichtbaar was met alle drie de kleurstoffen. Met de twee nieuwere kleurstoffen verscheen de ductus cysticus later, maar net zo goed als met IRDye ${ }^{\circledast} 800$ CW. De nieuwere IRDye ${ }^{\circledast} 800$ BK en IRDye ${ }^{\circledast} 800$ NOS lijken daarom goede alternatieven te zijn voor IRDye ${ }^{\circledast} 800 \mathrm{CW}$.

In hoofdstuk 5 worden de gebruikte methodes voor objectivering van het fluorescentiesignaal in de huidige literatuur geanalyseerd. We vonden dat de twee meest gebruikte softwareprogramma's (Osirix en ImageJ) vergelijkbare resultaten geven. Een derde softwareprogramma dat soms gebruikt wordt is Photoshop. Dit programma geeft minder goed vergelijkbare resultaten. Verder worden er in de huidige literatuur verschillende formules gebruikt om de fluorescentie-intensiteit van het doelorgaan af te zetten tegen de fluorescentie-intensiteit van de omliggende weefsels. De gebruikte formules zijn met elkaar vergeleken, maar er kan geen eenduidig antwoord gegeven worden welke formule te gebruiken. Wel is het advies om zowel de fluorescentie-intensiteit van het doelorgaan als de fluorescentie-intensiteit van de achtergrond te rapporteren om zo verschillende studieuitkomsten met elkaar te kunnen vergelijken in de toekomst. 


\section{DEEL 2 NABIJ-INFRARODE FLUORESCENTIE BEELDVORMING BIJ COLORECTALE CHIRURGIE}

Twee belangrijke complicaties bij colorectale chirurgie zijn naadlekkage nadat er een anastomose is aangelegd, en ureterschade bij chirurgie in het kleine bekken. Helaas is de incidentie van naadlekkage hoog, 3-15\% van colorectale operaties. ${ }^{14-21}$ Dit is een ernstige complicatie omdat het de morbiditeit verhoogt, er vaak een heroperatie nodig is, en er ook kans op mortaliteit is ${ }^{16,20-22} \mathrm{Er}$ zijn verschillende factoren van invloed op het voorkomen van naadlekkage bekend, waarvan de perfusie van de darmsegmenten die aan elkaar gemaakt worden een belangrijke is. ${ }^{23-25}$ Zonder goede perfusie van de anastomose zal deze niet goed genezen. Met het gebruik van NIRF-beeldvorming kan deze perfusie in beeld worden gebracht tijdens de operatie. Derhalve is de verwachting dat het gebruik van NIRF-angiografie tijdens de operatie een deel van de naadlekkages kan voorkomen. In hoofdstuk 6 hebben we deze hypothese onderzocht door middel van een systematische review en vonden we dat het gebruik van NIRF-angiografie in colorectale chirurgie makkelijk toepasbaar en veilig is. Na NIRF-angiografie werd de plaats van doornemen van de darmen verplaatst in $10.8 \%$ van de patiënten. Er werd significant minder frequent naadlekkage gezien in de gepubliceerde literatuur wanneer NIRF-angiografie werd gebruikt. Met name een initieel fel fluorescent signaal lijkt een voorspeller voor goede genezing van de anastomose.

In de studies geanalyseerd in hoofdstuk 6 werd de plaats van het doornemen van de darmen met name bepaald op basis van het subjectieve oordeel van de chirurg wanneer het signaal 'fluorescent genoeg' was. Er is geen duidelijke afkapwaarde wanneer het signaal fel genoeg is om zeker te zijn van voldoende perfusie, terwijl dit wel wenselijk is. In hoofdstuk 7 hebben we daarom onderzocht of de intensiteit van het fluorescente signaal samenhangt met serologische markers voor naadlekkage en postoperatieve inflammatoire markers zoals CRP, I-FABP en calprotectine. In een pilotstudie met een heterogene groep van 30 patiënten konden wij geen relatie vinden tussen fluorescentie-intensiteit en deze inflammatoire markers. Er werd wel een hogere fluorescentie-intensiteit van de darm gemeten in relatie tot de intensiteit van de achtergrond bij patiënten die geen naadlekkage ontwikkelden. Ook de subjectieve score voor fluorescentie-intensiteit van de darm was hoger in de patiënten die geen naadlekkage ontwikkelden.

Tijdens colorectale chirurgie kan de ureter zich erg dicht bij het operatiegebied bevinden en kan het moeilijk zijn om de ureter te onderscheiden van het andere omliggende weefsel. Toch is het herkennen van de locatie van de ureter de belangrijkste stap in het voorkomen van schade aan de ureter. Door gebruik te maken van een fluorescente kleurstof die wordt geklaard door de nieren kunnen de ureteren tijdens de operatie beter zichtbaar gemaakt worden. In hoofdstuk 8 werd het gebruik van methyleenblauw als fluorescente kleurstof om de ureter in beeld te brengen onderzocht in patiënten die electieve colorectale 
chirurgie ondergingen. Deze kleurstof is vrij beschikbaar en goedkoop. Een nadeel is echter dat deze kleurstof fluorescent is op een andere golflengte dan het veelgebruikte ICG, waarop de meeste apparatuur is ontworpen. Er zijn dus andere filters en software nodig om fluorescentie imaging met methyleenblauw te doen dan bij gebruik van ICG. In deze studie werden 10 patiënten geïncludeerd en werden verschillende doses methyleenblauw uitgeprobeerd. In vijf van de tien patiënten kon de ureter worden geïdentificeerd met methyleenblauw. Dit waren de patiënten die de hoogste dosis kregen, namelijk 0.75 tot $1 \mathrm{mg} / \mathrm{kg}$. In al deze vijf patiënten was de ureter in normaal (wit) licht ook zichtbaar. Vanwege deze teleurstellende resultaten lijkt het gebruik van methyleenblauw niet van toegevoegde waarde in de dagelijkse praktijk.

Een potente kleurstof met de excitatie en emissiegolflengte vergelijkbaar met die van ICG, die ook nog eens geklaard wordt door de nieren, zou ideaal zijn voor de beeldvorming van de ureters tijdens colorectale chirurgie. In hoofdstuk 9 en 10 worden twee nieuwe fluorescente kleurstoffen die aan deze eigenschappen voldoen, onderzocht. Dit zijn de preklinische dyes (IRDye ${ }^{\circledast} 800 B K$ en IRDye ${ }^{\circledast} 800$ NOS) welke door hun excitatie en emissie rond de $800 \mathrm{~nm}$ een veel grotere penetratiediepte hebben dan methyleenblauw met een excitatie en emissie net onder de 700nm. Deze nieuwe fluorescente kleurstoffen zijn getest in varkens en vergeleken met een eerder onderzochte preklinische stof: de IRDye ${ }^{\circledast} 800 \mathrm{CW}$. Elk varken kreeg één van deze stoffen intraveneus toegediend. Alle stoffen toonden een fel fluorescent signaal waarbij de ureters in de varkens zeer goed zichtbaar waren. Van de drie stoffen was het IRDye ${ }^{\oplus} 800 \mathrm{BK}$ welke het beste signaal gaf, en deze fluorescente kleurstof is daarom verder onderzocht in hoofdstuk 10. Drie verschillende doses van IRDye ${ }^{\circledR}$ $800 \mathrm{BK}$, namelijk 3mg, $6 \mathrm{mg}$ en $12 \mathrm{mg}$, werden toegediend in drie verschillende varkens. De resultaten verkregen met elk van de drie verschillende doses waren vergelijkbaar. Dezelfde kleurstof hebben we daarna nog gebruikt in geëxplanteerde humane en varkensureters om na te gaan of de penetratiediepte genoeg was om ook door de dikkere humane ureters de fluorescentie te kunnen zien. We observeerden dat dit mogelijk was, maar dat de aanwezigheid van vet op de ureter het fluorescente signaal wel duidelijk verminderde.

\section{DEEL 3 NABIJ-INFRARODE FLUORESCENTIE BEELDVORMING BIJ SCHILDKLIERCHIRURGIE}

Bij schildklierchirurgie is het belangrijk om schade aan de bijschildklieren te voorkomen. Ook hier is de belangrijkste factor in het voorkomen van iatrogene schade het identificeren van de anatomische structuur. Het is echter niet genoeg om enkel de bijschildklier te identificeren om schade aan de bijschildklier te voorkomen. Door manipulatie van de schildklier kan de bloedvoorziening naar de bijschildklier verminderd worden, waardoor de 
functie van een gespaarde bijschildklier alsnog onvoldoende is. In hoofdstuk 11 evalueren we zowel het identificeren van de bijschildklier als het beoordelen van de perfusie na het verwijderen van de schildklier. In 30 (hemi-)thyroidectomieën werd NIRF-beeldvorming gebruikt. De techniek was veilig en makkelijk toe te passen. In 17 van de 30 operaties werd het gebruik van NIRF-beeldvorming als toegevoegde waarde gezien door de operateur, met name door het geven van extra zekerheid over de locatie van de bijschildklier en de resterende functie. 


\section{REFERENCES}

1. Bobkiewicz A, Krokowicz L, Banasiewicz T, Koscinski T, Borejsza-Wysocki M, Ledwosinski W, et al. latrogenic bile duct injury. A significant surgical problem. Assessment of treatment outcomes in the department's own material. Pol Przegl Chir. 2014;86(12):576-83.

2. Boerma D, Rauws EA, Keulemans YC, Bergman JJ, Obertop H, Huibregtse K, et al. Impaired quality of life 5 years after bile duct injury during laparoscopic cholecystectomy: a prospective analysis. Ann Surg. 2001;234(6):750-7.

3. Booij KA, de Reuver PR, Yap K, van Dieren S, van Delden OM, Rauws EA, et al. Morbidity and mortality after minor bile duct injury following laparoscopic cholecystectomy. Endoscopy. 2015;47(1):40-6.

4. Dolan JP, Diggs BS, Sheppard BC, Hunter JG. Ten-year trend in the national volume of bile duct injuries requiring operative repair. Surg Endosc. 2005;19(7):967-73.

5. Fletcher DR, Hobbs MS, Tan P, Valinsky LJ, Hockey RL, Pikora TJ, et al. Complications of cholecystectomy: risks of the laparoscopic approach and protective effects of operative cholangiography: a population-based study. Ann Surg. 1999;229(4):449-57.

6. Flum DR, Dellinger EP, Cheadle A, Chan L, Koepsell T. Intraoperative cholangiography and risk of common bile duct injury during cholecystectomy. JAMA. 2003;289(13):1639-44.

7. Landman MP, Feurer ID, Moore DE, Zaydfudim V, Pinson CW. The long-term effect of bile duct injuries on health-related quality of life: a meta-analysis. HPB (Oxford). 2013;15(4):252-9.

8. Nuzzo G, Giuliante F, Giovannini I, Ardito F, D'Acapito F, Vellone M, et al. Bile duct injury during laparoscopic cholecystectomy: results of an Italian national survey on 56591 cholecystectomies. Archives of surgery (Chicago, III : 1960). 2005;140(10):986-92.

9. Waage A, Nilsson M. latrogenic bile duct injury: a population-based study of 152776 cholecystectomies in the Swedish Inpatient Registry. Archives of surgery (Chicago, III : 1960). 2006;141(12):1207-13.

10. Way LW, Stewart L, Gantert W, Liu K, Lee CM, Whang K, et al. Causes and prevention of laparoscopic bile duct injuries: analysis of 252 cases from a human factors and cognitive psychology perspective. Annals of surgery. 2003;237(4):460-9.

11. Strasberg SM, Hertl M, Soper NJ. An analysis of the problem of biliary injury during laparoscopic cholecystectomy. J Am Coll Surg. 1995;180(1):101-25.

12. Pesce A, Diana M. Critical View of Safety During Laparoscopic Cholecystectomy: From the Surgeon's Eye to Fluorescent Vision. Surg Innov. 2018;25(3):197-8.

13. Schols RM, Lodewick TM, Bouvy ND, van Dam DA, Meijerink WJ, van Dam GM, et al. Nearinfrared fluorescence laparoscopy of the cystic duct and artery in pigs: performance of a preclinical dye. J Laparoendosc Adv Surg Tech A. 2014;24(5):318-22.

14. Leahy J, Schoetz D, Marcello P, Read T, Hall J, Roberts P, et al. What is the risk of clinical anastomotic leak in the diverted colorectal anastomosis? Journal of gastrointestinal surgery: official journal of the Society for Surgery of the Alimentary Tract. 2014;18(10):1812-6.

15. Leichtle SW, Mouawad NJ, Welch KB, Lampman RM, Cleary RK. Risk factors for anastomotic leakage after colectomy. Diseases of the colon and rectum. 2012;55(5):569-75.

16. Midura EF, Hanseman D, Davis BR, Atkinson SJ, Abbott DE, Shah SA, et al. Risk factors and consequences of anastomotic leak after colectomy: a national analysis. Diseases of the colon and rectum. 2015;58(3):333-8.

17. Morse BC, Simpson JP, Jones YR, Johnson BL, Knott BM, Kotrady JA. Determination of independent predictive factors for anastomotic leak: analysis of 682 intestinal anastomoses. American journal of surgery. 2013;206(6):950-5; discussion 5-6.

18. Ruggiero R, Sparavigna L, Docimo G, Gubitosi A, Agresti M, Procaccini E, et al. Post-operative peritonitis due to anastomotic dehiscence after colonic resection. Multicentric experience, retrospective analysis of risk factors and review of the literature. Annali italiani di chirurgia. 2011;82(5):369-75. 
19. Sultan R, Chawla T, Zaidi M. Factors affecting anastomotic leak after colorectal anastomosis in patients without protective stoma in tertiary care hospital. JPMA The Journal of the Pakistan Medical Association. 2014;64(2):166-70.

20. Turrentine FE, Denlinger CE, Simpson VB, Garwood RA, Guerlain S, Agrawal A, et al. Morbidity, mortality, cost, and survival estimates of gastrointestinal anastomotic leaks. Journal of the American College of Surgeons. 2015;220(2):195-206.

21. Buchs NC, Gervaz P, Secic M, Bucher P, Mugnier-Konrad B, Morel P. Incidence, consequences, and risk factors for anastomotic dehiscence after colorectal surgery: a prospective monocentric study. International journal of colorectal disease. 2008;23(3):265-70.

22. Nachiappan S, Askari A, Currie A, Kennedy RH, Faiz O. Intraoperative assessment of colorectal anastomotic integrity: a systematic review. Surgical endoscopy. 2014;28(9):2513-30.

23. Karliczek A, Benaron DA, Zeebregts CJ, Wiggers T, van Dam GM. Intraoperative ischemia of the distal end of colon anastomoses as detected with visible light spectroscopy causes reduction of anastomotic strength. The Journal of surgical research. 2009;152(2):288-95.

24. Pommergaard HC, Achiam MP, Burcharth J, Rosenberg J. Impaired blood supply in the colonic anastomosis in mice compromises healing. International surgery. 2015;100(1):70-6.

25. Wu JH, Rong ZX, Zhu DJ, Chen XW, Ren BJ. [Laparoscopic anterior resection of rectal carcinoma with preservation of the left colonic artery]. Nan fang yi ke da xue xue bao = Journal of Southern Medical University. 2009;29(6):1249-50. 


\section{Valorisation}

Every surgical procedure incorporates a potential risk for complications. As described in the introduction of this thesis, part of these complications is caused by misidentification of the anatomy. A technique which improves intra-operative visualization of the anatomy could help avoid such complications, thereby prevent morbidity and mortality and lower health care costs. Near infrared fluorescence (NIRF) imaging concerns an innovative technique that might improve the intraoperative distinction of vital anatomical structures.

The aim of this thesis was to investigate a selection of the currently possible applications of NIRF imaging during surgery in order to prevent iatrogenic injury due to anatomical misrecognition.

\section{Relevance of scientific results in this thesis}

The use of the aforementioned technique (NIRF imaging) is aimed at preventing iatrogenic complications during surgery. Avoiding complications has multiple advantages. First, it prevents morbidity and, possibly, even mortality in patients. Second, complications are always accompanied with an increase in health care costs. As mentioned in the discussion of Chapter 6, the use of NIRF imaging is quite cheap, since indocyanine green costs only about 80 euros per vial, and for most applications, 1 vial can be used for multiple cases. The fluorescence modality is now incorporated in all new endoscopy systems and therefore does not result in additional costs. By preventing complications with a low-cost intervention, health care costs can be saved. Third, it is assumed that the use of NIRF imaging makes the surgical procedures easier. This means safer and faster, improving speed and outcome and thereby quality of care.

The three parts of this thesis cover four complications that are attempted to be prevented. The first complication is iatrogenic bile duct injury during laparoscopic cholecystectomy. In this case the use of NIRF imaging is applied for visualization of the cystic duct. We started a multicenter randomized controlled trial (Chapter 2) to investigate whether the use of NIRF imaging is actually as promising during elective laparoscopic cholecystectomies, as was suggested by earlier research. Next, in a systematic review combined with ex vivo experiments (Chapter 3) we found important factors to take into consideration while using the technique to obtain the best possible results. Also, a new fluorescent dye showed very promising images of the cystic duct, facilitating clear visualization of this structure during surgery (Chapter 4).

The second investigated complication is anastomotic leakage in colorectal surgery. Sufficient perfusion of the bowel at the location of the anastomosis is essential for 
anastomotic healing. By visualizing the bowel perfusion with NIRF imaging, it is expected that anastomotic leakage will occur less often. A systematic review was performed, indeed describing a lower anastomotic leak rate when using NIRF imaging (Chapter 6). Based on these results, the use of NIRF imaging to prevent anastomotic leakage is increasingly becoming standard practice. Our experiments show a possible relation between subjective and measured fluorescence intensity of the bowel using NIRF imaging and anastomotic leakage (Chapter 7), further supporting its use in practice.

The third complication we focused on is ureter damage in colorectal surgery. The use of NIRF imaging could facilitate visualization of the ureter. When the surgeon knows the exact location of the ureter, ureteral damage seems to be less likely. We showed that the ureter could be visualized using methylene blue as a fluorescent dye (Chapter 8), however, without added value compared to the naked eye. Novel dyes were tested in pigs and seemed more promising by providing a clear visualization of the ureters during surgery (Chapter 9 and 10).

In part four of the thesis, unwillingly parathyroid removal during thyroid surgery was the subject of investigation. Using NIRF imaging in thyroid surgery to identify the parathyroid glands provided more certainty about the location of the parathyroid glands (Chapter 11).

\section{Target population}

This thesis contributes to the patients requiring surgery, especially elective laparoscopic cholecystectomy, colorectal surgery or thyroid surgery. Based on the results of this thesis, other researchers and surgeons might be encouraged and inspired to further explore aspects of these applications, thereby improving these further and exploring other applications of the technique, helping patients undergoing other specific types of surgery.

\section{Innovation and future}

At the start of this thesis, the use of NIRF imaging was merely used in research settings. The protocol of the RCT described in Chapter $\mathbf{1}$ is the first started RCT regarding the intraoperative use of NIRF imaging. This RCT is the first step to assess whether the technique should be used in standard practice and should be incorporated in guidelines. The RCT in itself also improved the cooperation between surgeons, industry and research facilities since all these parties are actively involved in the RCT. However, although the RCT is an important step in the application of NIRF imaging, it might be unfit to illustrate the benefit of reduction of a complication with low frequency. This is the case with both bile duct injury and ureteral injury. The way to go here is prospective registration of all procedures performed in an international multicenter database. Our group is part of the international community of fluorescence imaging that is at present taking such initiatives. This community is also evaluating by several Delphi rounds the present status of application of the NIRF technique 
in any field that has been described so far. All these initiatives have in part been stimulated by the contribution of our group, by its research and by its active communication.

The use of methylene blue as a fluorescent dye seemed not of added value in this thesis. The excitation and emission wavelength of $\mathrm{MB}$ is different compared to the more successful indocyanine green. To visualize these dyes, a fluorescence imaging system providing the right excitation wavelength and filtering the right emission wavelength are needed. Since the use of MB was somewhat disappointing in our experiments, these wavelengths are not incorporated in standard fluorescence imaging systems. This led to the development of new dyes on the same excitation and emission wavelength as indocyanine green, so that standard fluorescence imaging systems can be used. This shows that the results of research in NIRF imaging influences the actions of the industry and the products that are developed and made commercially available.

During the progression of this thesis the use of NIRF imaging has become standard practice in several hospitals, mainly for the application of assessing perfusion during anastomotic bowel surgery.

One of the problems in assessing perfusion of anastomotic surgery is the decision when the bowel is 'fluorescent enough'. A start to answer this question was made in Chapter 7 of this thesis, although further research in a large cohort is needed to set a cutoff value of the fluorescence intensity that is ideally intra-operatively measured. Other future point of improvement of this technique include standard quantification of the signal (of which we made a start by writing Chapter 5), but also better dyes, better equipment and standard overlay of white and fluorescent light images

The application of NIRF imaging in thyroid surgery requires additional research to further assess the added value of the technique and improve the application. Because of the results produced in this thesis, one of the manufacturers of NIRF imaging systems is now re-evaluating their system to further improve the application of this technique in thyroid surgery. As soon as this system is available new research is required to re-assess the added value of this technique in thyroid surgery. 


\section{Dankwoord}

Ontzettend veel mensen hebben mij door deze promotie-tijd heen getrokken, geduwd en gelukkig ook soms gewoon enthousiast langs de lijn geapplaudisseerd voor de successen.

Professor Stassen, Laurents, ik wil jou ontzettend bedanken voor alles wat ik van jou heb mogen leren. Ik begon als WESP student, als een zenuwachtig meisje dat eigenlijk helemaal niet zo veel van onderzoek doen wist, en ook niet zo goed Engels kon. Met als resultaat heel veel 'rode tekst' in de protocollen die ik je toe stuurde. Gelukkig werden de teksten ook steeds minder rood, maar bleef jij wel het oog voor detail houden en is uiteindelijk elke komma in dit proefschrift weloverwogen geplaatst. Ik kon tijdens mijn promotie veranderen van dat zenuwachtige meisje naar een zelfverzekerde onderzoeker die met trots de resultaten presenteert op congressen; Waar jij dan ook zodra het maar enigszins kon halverwege in de zaal goedkeurend aan het knikken was bij wat ik zei. Ontzettend bedankt daarvoor!

Professor Bouvy, Nicole, als ik even het overzicht kwijt was, me onnodig zorgen maakte over dingen en gewoon even concrete afspraken en actieplannen nodig had was jij altijd daar. Met een oplossing. Jij hebt me geleerd dat er altijd een manier is om moeilijke dingen op te lossen, zelf danwel door de juiste persoon te zoeken die je daarbij kan helpen. Dat is een les die ik nooit zal vergeten.

Dr Schols, Rutger, jij was eigenlijk vanaf het moment dat ik me begon te verdiepen in de fluorescentie imaging mijn grote voorbeeld in dit veld. Ik denk dat ik jouw proefschrift misschien wel vaker heb gelezen dan jijzelf. Bij jou kon ik terecht voor de kleine dingen 'op welk knopje moest ik nu drukken?' en de grotere als het reviseren van de manuscripten en dit proefschrift. Onze reis naar Miami voor het fluorescentie congres zal me altijd bij blijven, en dan met name ons kort maar krachtige bezoek aan de Kennedy space center, de tour langs de highlights van Miami, de biertjes en burgers en natuurlijk onze fantastische praatjes die we daar gaven op het congres.

Fokko, officieel misschien niet in mijn promotieteam maar onofficieel ben jij dat eigenlijk wel een beetje. Jouw enthousiasme is ontzettend aanstekelijk. Na een kwartiertje met jou over het onderzoek praten kon ik zo weer 3 maanden door. Ik denk dat ik in de toekomst gewoon een paar projecten zal verzinnen waarbij jij nodig bent, alleen maar om weer met je samen te mogen werken. 
Mahdi, onze samenwerking verliep niet altijd vloeiend, maar ik denk wel dat we daarmee allebei uiteindelijk een stuk meer uit het onderzoek hebben kunnen halen. Al die uren die wij samen besteed hebben aan het analyseren van de videobeelden en het meten van de target to background ratio hebben zich uitbetaald in een aantal mooie publicaties, praatjes op congressen, een deel van dit proefschrift en ik weet zeker dat ook jouw proefschrift ook spoedig zal volgen.

Verder wil ik alle co-auteurs en iedereen die mee heeft geholpen aan de FALCON trial via deze weg bedanken. In het bijzonder Sander van Kuijk, wat fijn dat ik altijd bij jou terecht kon met mijn statistiek-vragen. Maar ook Jarno Melenhorst, Tim Lubbers en Sanne Engelen, Liza Mitalas en alle aiossen die de lap chols deden wil ik graag bedanken voor hun geduld op OK als ik weer eens hun patiënten had geïncludeerd in mijn studies.

Audrey, roooooomy! Wij deelde een werkkamer, soms wat studenten, af en toe onderzoeksprojecten en maar ook een afkeer voor veel dingen die voor andere de gewoonste zaak van de wereld waren. Dit maakte ons misschien soms wat gemeen, maar daar konden wij ook niets aan doen. Ik heb zo ontzettend veel lol met jou gehad, op het werk, op de fiets, in de zomer in het park, op congres en de korte vakanties die we daar soms aan vast plakte. Met jou waren zelfs vage congressen in the middle of nowhere van Tsjechië één groot feest.

Sebastiaan, Steen, jouw foute-uur afspeellijst heeft me door de laatste loodjes van deze promotie heen getrokken. In combinatie met onze ochtend-koffie, lunch-koffie en middagkoffie natuurlijk. Wald, Waldo, wat een feest is alles als jij er ook bij bent! (Behalve als we 30 seconds spelen, dat doe ik nooit meer met of tegen jou). Ons zeilweekendje was een van de vele hoogtepunten tijdens deze tijd, maar ook de feestjes, jouw Daniel Arends imitaties, de avonturen op de wielrenfiets en op het trailrun-pad. Cathelijne, Cath (geen kat want katten zijn niet jouw ding). Ik heb nog spierpijn van het lachen, denk niet dat die ooit nog weg gaat. Jouw doorzettingsvermogen en enthousiasme zijn inspirerend en aanstekelijk. Rob, met jou fietsen heet niet voor niets 'huilen met Rob'. Gelukkig breng je naast wat tranen vooral veel gezelligheid, mooie routes en ben jij een ontzettend goede organisator van lab-uitjes, sinterklaasfeestjes, wielertours op groot scherm kijken en eigenlijk zo'n beetje alle leuke dingen die er op het lab gebeurde. Met jullie vier zeilen was één van de leukste weekendjes weg die ik ooit heb gedaan. Maar ik zal ook mijn tafelvoetbal-skills nooit meer verliezen na al die uren training met jullie.

Marissa, jij begon ongeveer tegelijkertijd met mij vanuit de semi stage als promovendus op het lab. 's avonds of tijdens de koffie konden wij uren praten, roddelen en ons verbazen over onze nieuwe collega's, METC, onze grote RCTs en de wereld van de chirurgen. Op de chirurgendagen deelde wij vanzelfsprekend een kamer met 8 mensen en probeerde we zo 
het 'mamma-team' en de rest van de chirurgie-promovendi enigszins te verbinden. Romy, de hoeveelheid energie die jij hebt is ongekend. Ik bewonder jou enorm om de manier waarop jij zelf beslist wat je belangrijk vindt in het leven en er ook nog eens voor zorgt dat jij al die dingen gewoon allemaal tegelijk kan doen.

Leontine, Kim, Bas, David, Martine, Yvonne, Frans en Jasper jullie waren er vanaf het begin van mijn promotie-onderzoek bij en ik ben ontzettend blij met jullie ontvangst in de groep. Ook het licht dwingende advies om een wielrenfiets te kopen, was misschien wel een van de beste adviezen in mijn leven. Ik wil jullie ontzettend bedanken voor de gezellige tijd op het lab, op de fiets in het prachtige heuvelland en tijdens de vrijdagmiddagborrels waar jullie toch wel een van de key-ingredients voor waren.

Claire, Mirjam, Rianne, Merel, Cathy, jullie waren/zijn de échte lab-mensen. Met grote verbazing ontvingen jullie mij soms in het lab als ik af en toe een buisje voor Audrey moest afdraaien. Wat blijkbaar heel simpel is, maar waarbij ik dan meestal toch jullie hulp een beetje nodig had, waarvoor dank.

Collega's uit het Viecuri: Michael, Frans, Anne, Stan, Maarten, Freek, Maud, Martijn, Tim, Tom, Marlies, Rutger, Eliza, Alexandra, Mintsje, Melissa; in het jaar, of voor sommige enkele maanden dat ik met jullie samen heb mogen werken heb ik het enorm naar mijn zin gehad. Jullie hebben mij de eerste stapjes als dokter (niet doctor) helpen maken. Julie waren allemaal ontzettend fijne collega's! Ook mijn nieuwe collega's bij de KNO in het Zuyderland ontzettend bedanken voor de kansen die jullie mij geven om dit vak te ontdekken en de fijne nieuwe werkplek.

Lieve angels, who the $\mathrm{F}^{* * *}$ is Els? En waarom staat ze in dit dankwoord? Geen idee. Jullie heb ik grotendeels pas na mijn fulltime promotie-tijd leren kennen, maar jullie hebben er wel voor gezorgd dat ik voldoende energie had voor de laatste loodjes van dit proefschrift. En jullie zorgen er natuurlijk voor dat ik nooit meer van de triatlon-verslaving af zal komen zolang jullie ook mee doen. Ik doe mijn uiterste best om jullie soort van bij te blijven houden.

Lieve Malou, wat ben ik blij dat ik jou heb leren kennen. Het is super om iemand te hebben die over zo veel dingen hetzelfde denkt, dezelfde kledingsmaak heeft, maar dan net een ander figuur waardoor de leuke dingen die jou niet perfect staan mij vaak wel en andersom, even fanatiek is en gewoon snapt wat ik wil, wens en bedoel. Ik vind het ontzettend jammer dat je zo ver weg woont maar ben ook heel erg blij jij desondanks zo'n goede vriendin voor mij blijft. 
Lieve Aline, wat mis ik ons borrel-plank en wijn diner op zondag af en toe, zoals wij dat deden in de studententijd. Ook mis ik soms jouw al dan niet mislukte baksels, tripjes naar Parijs, Disney en Milaan. Gelukkig heb ik daar af en toe gezellige telefoontjes, appjes en dates ergens tussen Den Haag en Maastricht voor terug gekregen en ben ik vooral heel blij voor jou dat alles voor zover gelukt is waar je op gehoopt had.

Leslie, wat hou ik van de luie zondagmiddagen waarin we lekker uit lunchen gaan en door een stad wandelen. Nog even wachten en dan kunnen we met de kinderwagen met daarin jouw mooie kindje door die steden lopen. Anne, met jou lekker de natuur in op de fiets of met de hardloopschoenen is zo heerlijk ontspannend. Daarna kan ik alles weer aan. Kimberley, Hester en Marloes, wat ken ik jullie al lang zeg! We spreken elkaar niet heel vaak meer maar ik vind het altijd super gezellig om bij jullie te zijn.

Mijn schoonfamilie, Carroll, Marianne, wat fijn dat ik altijd bij jullie terecht kan en zo vaak van jullie kook-kunsten mag genieten.

Paps, mams en Stefan, ookal kon ik soms niet goed uitleggen wat ik nou de hele dag deed. Jullie en (vooral paps) snapte wel goed dat dit proefschrift een keer af moest. Door jouw subtiele vragen 'heb je al een datum?' werd menig treinreis met een goed boek toch veranderd in een reis waarin op de laptop werd gewerkt aan een manuscript. Ook was hotel mamma altijd beschikbaar als ik weer eens een slaapplek nodig had voor congres of reis, of gewoon voor de gezelligheid. Ik hoop dat ik jullie trots maak met wat ik doe en dat jullie je realiseren dat jullie daar altijd, al is het soms op afstand, een steentje aan bij hebt gedragen.

Lieve Casper, Cas, eigenlijk ben jij de reden dat ik überhaupt ben gaan nadenken over het doen van promotieonderzoek. Jouw enthousiasme en vastberadenheid over de toegevoegde waarde van het doen van onderzoek heeft mij aan het denken gezet dat ik dat dan misschien ook wel wilde, en heeft ervoor gezorgd dat ik me vanaf het begin als een pitbull in deze promotieplek heb vastgebeten. Op de moeilijke tijden was jouw schouder er om even op uit te huilen, bij de kleine en grote successen was jij er om het glas te heffen. Die researchgate score van mij zal denk ik nooit hoger worden dan die van jou, but one can always dream... Ik hoop dat jij er altijd voor mij zou willen zijn als schouder om op uit te huilen en als liefste om het glas mee te heffen in goede tijden. Want samen met jou zijn de zware tijden minder zwaar en de goede tijden het best. 


\section{List of Publications}

Jacqueline van den Bos, Rutger M. Schols, Sander van Kuijk, Fokko Wieringa, Laurents P.S. Stassen. Technical note: are currently used measurements of fluorescence intensity in near infrared fluorescence imaging during laparoscopic cholecystectomy comparable? J Laparoendosc Adv Surg Tech A. 2019

Tim Pruimboom, Sander MJ van Kuijk, Shan Shan Qiu Sao, Jacqueline van den Bos, Fokko P Wieringa, Renee RWJ van der Hulst, Rutger M Schols. Optimizing Indocyanine Green Fluorescence Angiography in Reconstructive Flap Surgery: A Systematic Review and Ex Vivo Experiments. Surg Innov. 2019

Vincent Agnus, Antonio Pesce, Luigi Boni, Jacqueline van den Bos, Salvador Morales-Conde, Alessandro M. Paganini, Silvia Quaresima, Andrea Balla, Gaetano La Greca, Haralds Plaudis, Gianluigi Moretto, Maurizio Castagnola, Caterina Santi, Lorenzo Casali, Luciano Tartamella, Alend Saadi, Andrea Picchetto, Alberto Arezzo, Jacques Marescaux, Michele Diana. Fluorescencebased cholangiography: preliminary results from the IHU-IRCAD-EAES EURO-FIGS registry. Accepted at Surgical Endoscopy 2019

Jacqueline van den Bos, Audrey C.H.M. Jongen, Jarno Melenhorst, Stéphanie O. Breukink, Kaatje Lenaerts, Rutger M. Schols, Nicole D. Bouvy, Laurents P.S. Stassen. Near-infrared fluorescence image-guidance in anastomotic colorectal cancer surgery and its relation to serum markers of anastomotic leakage: a clinical pilot study. Surgical Endoscopy. 2019

Jacqueline van den Bos, Lottie van Kooten, Sanne M.E. Engelen, Tim Lubbers, Laurents P.S. Stassen, Nicole D. Bouvy. Feasibility of indocyanine green fluorescence imaging for intraoperative identification of parathyroid glands during thyroid surgery. Head and Neck. 2018

Jacqueline van den Bos, Mahdi Al-Taher, Nicole D. Bouvy, Laurents P.S. Stassen. Near-infrared fluorescence laparoscopy of the ureter with three preclinical dyes in a pig model. Surgical Endoscopy. 2018

Sebastiaan van Steensel, Arthur Bloemen, Leontine C.L. van den Hil, Jacqueline van den Bos, Gert-Jan Kleinrensink, Nicole D. Bouvy. Pitfalls and clinical recommendations for the primary lumbar hernia based on a systematic review of the literature. Hernia. 2018

Jacqueline van den Bos, Fokko P. Wieringa, Nicole D. Bouvy, Laurents P.S. Stassen. Optimizing the image of fluorescence cholangiography using ICG: a systematic review and ex vivo experiments. Surgical Endoscopy. 2018 
Mahdi Al-Taher, Jacqueline van den Bos, Rutger M. Schols, Bela Kubat, Nicole D. Bouvy, Laurents P.S. Stassen. Evaluation of a novel dye for near-infrared fluorescence delineation of the ureters during laparoscopy. BJS Open. 2018

Floyd W. van de Graaf, Jacqueline van den Bos, Laurents P.S. Stassen, Johan F. Lange. Lacunar implementation of the critical view of safety technique for laparoscopic cholecystectomy: Results of a nationwide survey. Surgery. 2018

Jacqueline van den Bos, Mahdi Al-Taher, Rutger M. Schols, Sander M.J. van Kuijk, Nicole D. Bouvy, Laurens P.S. Stassen. Near-Infrared Fluorescence Imaging for Real-Time Intraoperative Guidance in Anastomotic Colorectal Surgery: A Systematic Review of Literature. J Laparoendosc Adv Surg Tech A. 2017

Claudius Conrad, Go Wakabayash, Horacio J. Asbun, Bernard Dallemagne, Nicolas Demartines, Michele Diana, David Fuks, Mariano Eduardo Gimenez, Claire Goumard, Hironori Kaneko, Riccardo Memeo, Alexandre Resende, Olivier Scatton, Anne-Sophie Schneck, Olivier Soubrane, Minoru Tanabe, Jacqueline van den Bos, Helmut Weiss, Masakazu Yamamoto, Jacques Marescaux, Patrick Pessaux. IRCAD recommendation on safe laparoscopic cholecystectomy. J Hepatobiliary Pancreat Sci. 2017

Jacqueline van den Bos, Mahdi Al-Taher, Shu Gi Hsien, Nicole D. Bouvy, Laurents P.S. Stassen. Near infrared fluorescence laparoscopy of the cystic duct and cystic artery: first experience with two new preclinical dyes in a pig model. Surgical Endoscopy. 2017

Jacqueline van den Bos, Rutger M. Schols, Misha D. Luyer, Ronald M. van Dam, Alexander L. Vahrmeijer, Wilhelmus J. Meijerink, Paul D. Gobardhan, Gooitzen M. van Dam, Nicole D. Bouvy, Laurents P.S. Stassen. Near-infrared fluorescence cholangiography assisted laparoscopic cholecystectomy versus conventional laparoscopic cholecystectomy (FALCON trial): study protocol for a multicentre randomised controlled trial. BMJ Open. 2016

Mahdi Al-Taher, Jacqueline van den Bos, Rutger M. Schols, Nicole D. Bouvy, Laurents P.S. Stassen. Fluorescence Ureteral Visualization in Human Laparoscopic Colorectal Surgery Using Methylene Blue. Journal of laparoendoscopic \& advanced surgical techniques Part A. 2016

Sofie A. Fransen, Jacqueline van den Bos, Laurents P.S. Stassen, Nicole D. Bouvy. Is SinglePort Laparoscopy More Precise and Faster with the Robot? Journal of laparoendoscopic \& advanced surgical techniques Part A. 2016 


\section{Curriculum vitae}

Jacqueline van den Bos was born on April $24^{\text {th }}, 1990$ in Leiderdorp, The Netherlands. After graduating from secondary school at the 'Da Vinci College' in Leiden in the summer of 2008, she started the study Health Sciences at Maastricht University, from which she completed the first year and followed additional courses in the second and third year from the minor 'Mental Health Sciences'. In 2009 she started medical school at Maastricht University. During the first year of the Bachelor's program of medicine, she was a student member of the Faculty Council of the Faculty of Health Medicine and Life Sciences at Maastricht University and participated in multiple committees of her student association (M.S.R.V Saurus, Maastricht). She received a bachelor's degree in Medicine in the summer of 2012 and preceded to the Masters program and graduated on July 31st, 2015. During the final year of Medical school, she did her research internship under the supervision of professor dr. N.D. (Nicole) Bouvy and professor L.P.S. (Laurents) Stassen, investigating the added value of the surgical robot in single port laparoscopy and later also the use of near infrared fluorescence imaging to identify the ureter. This research triggered her attention for research in general and the particular topic of near infrared fluorescence imaging. The research internship was therefore followed by this $\mathrm{PhD}$ project and she was employed as a $\mathrm{PhD}$ candidate at the department of Surgery at Maastricht University/MUMC+ from August 2015 until July 2018 under the supervision of professor Stassen and Bouvy. From July 2018 until July 2019, she worked as a surgical resident (ANIOS) at VieCuri Medisch Centrum. Since September 2019 she is a resident (ANIOS) in Ear, Nose, and Throat surgery at the Zuyderland Medisch Centrum. 

